\title{
Observed galaxy number counts on the lightcone up to second order: II. Derivation
}

\author{
Daniele Bertacca $^{a}$, Roy Maartens ${ }^{a, b}$, Chris Clarkson $^{c}$ \\ ${ }^{a}$ Physics Department, University of the Western Cape, Cape Town 7535, South Africa \\ ${ }^{b}$ Institute of Cosmology \& Gravitation, University of Portsmouth, Portsmouth PO1 3FX, UK \\ ${ }^{c}$ Centre for Astrophysics, Cosmology \& Gravitation, \\ and, Department of Mathematics \& Applied Mathematics, \\ University of Cape Town, Cape Town 7r01, South Africa
}

(Dated: October 22, 2018)

\begin{abstract}
We present a detailed derivation of the observed galaxy number over-density on cosmological scales up to second order in perturbation theory. We include all relativistic effects that arise from observing on the past lightcone. The derivation is in a general gauge, and applies to all dark energy models (including interacting dark energy) and to metric theories of modified gravity. The result will be important for accurate cosmological parameter estimation, including non-Gaussianity, since all projection effects need to be taken into account. It also offers the potential for new probes of General Relativity, dark energy and modified gravity. This paper accompanies Paper I which presents the key results for the concordance model in Poisson gauge.
\end{abstract}

\section{INTRODUCTION}

The Newtonian prediction for the galaxy number over-density is accurate only on small scales. On cosmological scales, relativistic effects alter the observed number over-density through projection onto our past lightcone. This gives the well-known corrections from redshift space distortions and gravitational lensing convergence, but there are further Doppler, Sachs-Wolfe, integrated SW and time-delay type terms. The full relativistic effects have been calculated to first order in perturbation theory by [1 5 . The nature, importance and possible detectability of the relativistic corrections to the Newtonian approximation at first order have been considered by [6]-[19].

We derive the formula for the observed galaxy number over-density up to second order on cosmological scales. The result in Poisson gauge and for the concordance model is presented in the companion Paper I 20]. Paper I also considers the implications of the result for cosmological observables. Here we give the detailed derivation both in a general gauge and in Poisson gauge, for a flat Roberston-Walker background which allows for general dark energy models, including those where dark energy interacts non-gravitationally with cold dark matter. These interacting models have momentum exchange between dark energy and cold dark matter, which can lead to a velocity bias between galaxies and cold dark matter. Our results allow for velocity bias.

Our results also apply to metric theories of modified gravity as an alternative to dark energy, since we do not impose any field equations (in particular, we do not assume that the gauge invariant metric perturbations $\Phi$ and $\Psi$ are equal). The main result contains all relativistic effects up to second order that arise from observing on the past light cone, including all redshift and lensing distortions - due to convergence, shear and rotation - and all contributions from velocities, SW, ISW and time-delay terms (for example, see [21, 22]). This is related to the second-order perturbations of the cosmological distance-redshift relation [23]-32, and to the weak lensing shear up to second order [33, 34.

The second-order effects that we derive, especially those involving integrals along the line of sight, may make a non-negligible contribution to the observed number counts. This could be important for removing bias on parameter estimation in precision cosmology with galaxy surveys. Recently, it has been shown that second-order effects on the distance-redshift relation induce a shift in the background that may have a significant effect on estimates of the Hubble constant and matter density parameter [31, 32].

We follow the "cosmic rulers" approach of [5, 7, generalizing it from first to second order. Consequently, we use only the observed redshift $z$ in our analysis. In particular, all background quantities are evaluated at the observed, not background, redshift. Thus we do not need to identify the perturbations of redshift (these are derived in full detail up to second order by [23, 26]). We neglect magnification bias, leaving this for future work [27.

The paper is organised as follows: in Section [II we briefly review and generalize the cosmic rulers from first to second order, and apply it to obtain the second-order perturbations of galaxy number counts in redshift space. We perturb a flat Robertson-Walker universe in a general gauge in Section III and specialize to Poisson Gauge in Section IV. In Section $[\mathrm{V}$ we describe how to relate the fluctuations of galaxy number density to the underlying matter density fluctuation $\delta_{m}$. Finally, Section VI is devoted to conclusions.

The derivation of the second-order solutions is careful to include all steps needed for an independent verification of the results. It is therefore lengthy and technical.

- For readers who want to understand the derivation of the general formula, see Section II and Section III. The 
main results are Eqs. 158 and 159 .

- For readers who are happy to skip the proof and look at the main result for galaxy number count fluctuations at second order in the Poisson gauge - see Paper I for the concordance model, or Section [V] for general dark energy and modified gravity models. The main results are Eqs. 237) and Eq. 238.

Conventions: units $c=G=1$; signature is $(-,+,+,+)$; Greek indices run over $0,1,2,3$, and Latin over $1,2,3$.

Note added in version 4: In the previous version 3 (and in the published paper), we mistakenly omitted some terms which arise from the integration of a first-order quantity taking into account perturbations of the direction of the null geodesic (so-called post-Born terms). This error has been corrected here and in the companion paper 1405.4403v5. Our results are now in agreement, in the appropriate limit, with those of [28].

\section{COSMIC RULERS AND THE OBSERVED OVERDENSITY}

The cosmic rulers formalism of [5, 7] provides a map between redshift-space and real-space, without introducing a metric. Here we generalize it to second order. We denote quantities in the redshift frame with a bar.

Redshift-space is the "cosmic laboratory" where we probe the observations. We perform perturbations in real-space and not in redshift-space. In redshift-space we use coordinates which effectively flatten our past lightcone so that the photon geodesic from an observed galaxy has the following conformal space-time coordinates (see Fig. 1):

$$
\bar{x}^{\mu}=(\bar{\eta}, \overline{\mathbf{x}})=\left(\eta_{0}-\bar{\chi}, \bar{\chi} \mathbf{n}\right)
$$

Here $\bar{\chi}(z)$ is the comoving distance to the observed redshift in redshift-space, calculated in the background, and $\mathbf{n}$ is the observed direction to the galaxy, $n^{i}=\bar{x}^{i} / \bar{\chi}=\delta^{i j}\left(\partial \bar{\chi} / \partial \bar{x}^{j}\right)$. Using $\bar{\chi}$ as an affine parameter, the total derivative

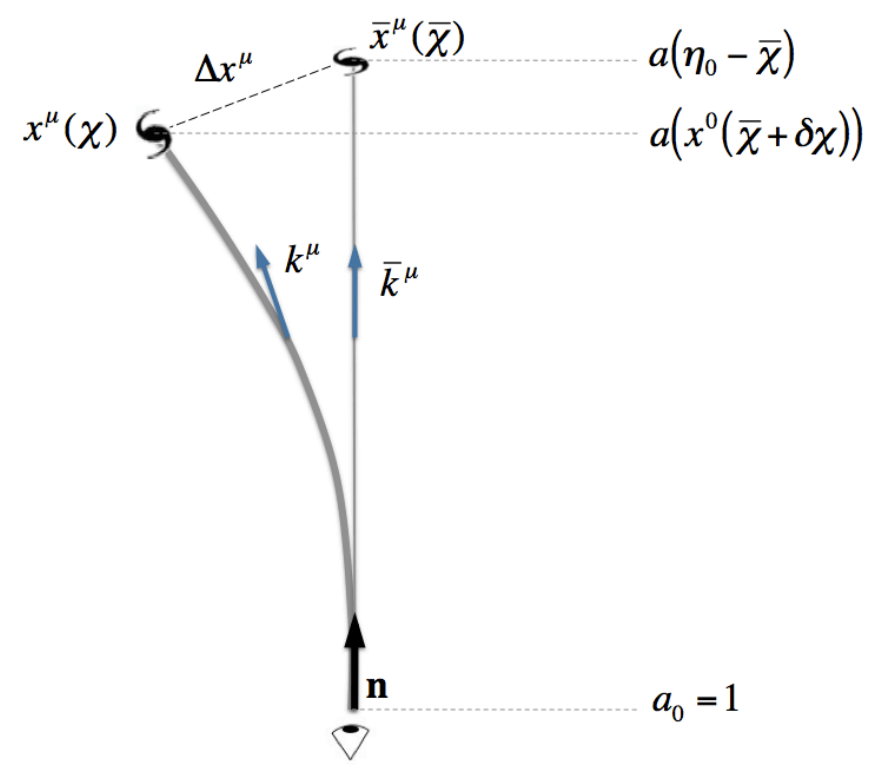

FIG. 1: The real-space and redshift-space views (adapted from [7]).

along the past light cone is

$$
\frac{\mathrm{d}}{\mathrm{d} \bar{\chi}}=-\frac{\partial}{\partial \bar{\eta}}+n^{i} \frac{\partial}{\partial \bar{x}^{i}} .
$$

To map from redshift-space to real-space (the "physical frame"), we introduce coordinates $x^{\mu}=x^{\mu}(\chi)$, where $\chi$ is the physical comoving distance of the source (see Fig. 1). Then, to second order

$$
x^{\mu}(\chi)=\bar{x}^{\mu}(\chi)+\delta x^{\mu}(\chi) \quad \text { where } \quad \delta x^{\mu}(\chi)=\delta x^{\mu(1)}(\chi)+\frac{1}{2} \delta x^{\mu(2)}(\chi) .
$$


We map the real-space frame to the redshift frame perturbatively via

$$
x^{\mu}(\chi)=\bar{x}^{\mu}(\bar{\chi})+\Delta x^{\mu}(\bar{\chi}) \quad \text { where } \quad \Delta x^{\mu}(\bar{\chi})=\Delta x^{\mu(1)}(\bar{\chi})+\frac{1}{2} \Delta x^{\mu(2)}(\bar{\chi}) .
$$

The physical comoving distance to the source is a perturbation about the value in the redshift frame:

$$
\chi=\bar{\chi}+\delta \chi \quad \text { where } \quad \delta \chi=\delta \chi^{(1)}+\frac{1}{2} \delta \chi^{(2)} .
$$

Then we have

$$
\begin{aligned}
x^{\mu}(\chi) & =\bar{x}^{\mu}(\chi)+\delta x^{\mu(1)}(\chi)+\frac{1}{2} \delta x^{\mu(2)}(\chi) \\
& =\bar{x}^{\mu}(\bar{\chi})+\frac{\mathrm{d} \bar{x}^{\mu}}{\mathrm{d} \bar{\chi}} \delta \chi^{(1)}+\delta x^{\mu(1)}(\bar{\chi})+\frac{1}{2} \frac{\mathrm{d} \bar{x}^{\mu}}{\mathrm{d} \bar{\chi}} \delta \chi^{(2)}(\bar{\chi})+\frac{1}{2} \frac{\mathrm{d}^{2} \bar{x}^{\mu}}{\mathrm{d} \bar{\chi}^{2}}\left(\delta \chi^{(1)}\right)^{2}+\frac{\mathrm{d} \delta x^{\mu(1)}}{\mathrm{d} \bar{\chi}} \delta \chi^{(1)}+\frac{1}{2} \delta x^{\mu(2)}(\bar{\chi}),
\end{aligned}
$$

which implies

$$
\begin{aligned}
& \Delta x^{\mu(1)}(\bar{\chi})=\frac{\mathrm{d} \bar{x}^{\mu}}{\mathrm{d} \bar{\chi}} \delta \chi^{(1)}+\delta x^{\mu(1)}(\bar{\chi}) \\
& \Delta x^{\mu(2)}(\bar{\chi})=\frac{\mathrm{d} \bar{x}^{\mu}}{\mathrm{d} \bar{\chi}} \delta \chi^{(2)}+\frac{\mathrm{d}^{2} \bar{x}^{\mu}}{\mathrm{d} \bar{\chi}^{2}}\left(\delta \chi^{(1)}\right)^{2}+2 \frac{\mathrm{d} \delta x^{\mu(1)}}{\mathrm{d} \bar{\chi}} \delta \chi^{(1)}+\delta x^{\mu(2)}(\bar{\chi})
\end{aligned}
$$

The photon 4-momentum is

$$
p^{\mu}=\frac{\nu(a)}{a} k^{\mu}
$$

where $a$ is the scale factor, $\nu \propto 1 / a$ is the frequency in a homogeneous and isotropic space-time, and $k^{\mu}$ is a null geodesic vector. In the redshift frame

$$
\bar{k}^{\mu}=\frac{\mathrm{d} \bar{x}^{\mu}}{\mathrm{d} \bar{\chi}}=(-1, \mathbf{n})
$$

while the physical $k^{\mu}$ evaluated at $\bar{\chi}$ is

$$
k^{\mu}(\bar{\chi})=\frac{\mathrm{d} x^{\mu}}{\mathrm{d} \bar{\chi}}(\bar{\chi})=\frac{\mathrm{d}}{\mathrm{d} \bar{\chi}}\left(\bar{x}^{\mu}+\delta x^{\mu}\right)(\bar{\chi})=\left(-1+\delta \nu^{(1)}+\frac{1}{2} \delta \nu^{(2)}, n^{i}+\delta n^{i(1)}+\frac{1}{2} \delta n^{i(2)}\right)(\bar{\chi}) .
$$

For $\mu=0$ we have

$$
\begin{aligned}
& \Delta x^{0(1)}(\bar{\chi})=-\delta \chi^{(1)}+\delta x^{0(1)} \\
& \Delta x^{0(2)}(\bar{\chi})=-\delta \chi^{(2)}+2 \delta \nu^{(1)} \delta \chi^{(1)}+\delta x^{0(2)}
\end{aligned}
$$

where $\mathrm{d} \delta x^{0(n)} / \mathrm{d} \bar{\chi}=\delta \nu^{(n)}$, and for $\mu=i$,

$$
\begin{aligned}
& \Delta x^{i(1)}(\bar{\chi})=n^{i} \delta \chi^{(1)}+\delta x^{i(1)} \\
& \Delta x^{i(2)}(\bar{\chi})=n^{i} \delta \chi^{(2)}+2 \delta n^{i(1)} \delta \chi^{(1)}+\delta x^{i(2)} .
\end{aligned}
$$

where $\mathrm{d} \delta x^{i(n)} / \mathrm{d} \bar{\chi}=\delta n^{i(n)}$. From Eq. 11, , we obtain explicitly

$$
\begin{aligned}
& \delta x^{0(n)}(\bar{\chi})=\int_{0}^{\bar{\chi}} \mathrm{d} \tilde{\chi} \delta \nu^{(n)}(\tilde{\chi}), \\
& \delta x^{i(n)}(\bar{\chi})=\int_{0}^{\bar{\chi}} \mathrm{d} \tilde{\chi} \delta n^{i(n)}(\tilde{\chi}),
\end{aligned}
$$

where we have imposed the boundary conditions at the observer: $\delta x_{o}^{0(n)}=0$ and $\delta x_{o}^{i(n)}=0$. 


\section{A. The scale factor}

In real-space the scale factor is

$$
a=a\left(x^{0}(\chi)\right)=a\left(\bar{x}^{0}+\Delta x^{0}\right)=\bar{a}\left[1+\mathcal{H} \Delta x^{0(1)}+\frac{1}{2} \mathcal{H} \Delta x^{0(2)}+\frac{1}{2}\left(\mathcal{H}^{\prime}+\mathcal{H}^{2}\right)\left(\Delta x^{0(1)}\right)^{2}\right]
$$

where $\bar{a}=a\left(\bar{x}^{0}\right)$, prime is $\partial / \partial \bar{x}^{0}=\partial / \partial \bar{\eta}$ and $\mathcal{H}=\bar{a}^{\prime} / \bar{a}$. Defining

$$
\frac{a}{\bar{a}}=1+\Delta \ln a^{(1)}+\frac{1}{2} \Delta \ln a^{(2)}
$$

we find

$$
\begin{aligned}
\Delta \ln a^{(1)} & =\mathcal{H} \Delta x^{0(1)}=\mathcal{H}\left(-\delta \chi^{(1)}+\delta x^{0(1)}\right) \\
\Delta \ln a^{(2)} & =\left(\mathcal{H}^{\prime}+\mathcal{H}^{2}\right)\left(\Delta x^{0(1)}\right)^{2}+\mathcal{H} \Delta x^{0(2)}=\frac{\left(\mathcal{H}^{\prime}+\mathcal{H}^{2}\right)}{\mathcal{H}^{2}}\left(\Delta \ln a^{(1)}\right)^{2}+\mathcal{H} \Delta x^{0(2)} \\
& =\left(\mathcal{H}^{\prime}+\mathcal{H}^{2}\right)\left(-\delta \chi^{(1)}+\delta x^{0(1)}\right)^{2}-\mathcal{H} \delta \chi^{(2)}+2 \mathcal{H} \delta \nu^{(1)} \delta \chi^{(1)}+\mathcal{H} \delta x^{0(2)} .
\end{aligned}
$$

\section{B. Four-vectors and tetrads}

The galaxy four-velocity can be given as

$$
u^{\mu}=\frac{\mathrm{d} x^{\mu}}{\mathrm{d} s}=\frac{\mathrm{d} x^{\hat{\alpha}}}{\mathrm{d} s} \Lambda_{\hat{\alpha}}^{\mu}=u^{\hat{\alpha}} \Lambda_{\hat{\alpha}}^{\mu},
$$

where $s$ is proper time and $\Lambda_{\hat{\alpha}}^{\mu}$ is an orthonormal tetrad. If we choose $u^{\mu}$ as the timelike basis vector, then

$$
u_{\mu}=\Lambda_{\hat{0} \mu}=a E_{\hat{0} \mu} \quad \text { and } \quad u^{\mu}=\Lambda_{\hat{0}}^{\mu}=a^{-1} E_{\hat{0}}^{\mu},
$$

where $E_{\hat{\alpha}}^{\mu}$ is the tetrad in the comoving frame. In the background

$$
E_{\hat{0} \mu}^{(0)}=(-1, \mathbf{0})
$$

and perturbing, we obtain

$$
E_{\hat{0} \mu}\left(x^{\nu}(\chi)\right)=E_{\hat{0} \mu}\left(\bar{x}^{\nu}(\bar{\chi})+\Delta x^{\nu}\right)=E_{\hat{0} \mu}^{(0)}(\bar{\chi})+E_{\hat{0} \mu}^{(1)}(\bar{\chi})+\left(\frac{\partial E_{\hat{0} \mu}}{\partial \bar{x}^{\nu}}\right)^{(1)} \Delta x^{\nu(1)}+\frac{1}{2} E_{\hat{0} \mu}^{(2)}(\bar{\chi}) .
$$

From $k^{\mu}$, the map from redshift to real-space is given by

$$
k^{\mu}(\chi)=\frac{\mathrm{d} x^{\mu}(\chi)}{\mathrm{d} \chi}=k^{\mu}(\bar{\chi}+\delta \chi)=k^{\mu(0)}(\bar{\chi})+k^{\mu(1)}(\bar{\chi})+\left(\frac{\mathrm{d} k^{\mu}}{\mathrm{d} \bar{\chi}}\right)^{(1)} \delta \chi^{(1)}+\frac{1}{2} k^{\mu(2)}(\bar{\chi})
$$

where $k^{\mu(0)}(\bar{\chi})=\bar{k}^{\mu}$.

\section{The observed redshift}

The observed redshift is given by

$$
\left.(1+z)\right|_{\chi}=\frac{\left.\left(u_{\mu} p^{\mu}\right)\right|_{\chi}}{\left.\left(u_{\mu} p^{\mu}\right)\right|_{o}}=\frac{\nu(\chi)}{\nu_{o}} \frac{\left.\left(E_{\hat{0} \mu} k^{\mu}\right)\right|_{\chi}}{\left.\left(E_{\hat{0} \mu} k^{\mu}\right)\right|_{o}}=\frac{a_{o}}{a(\chi)} \frac{\left.\left(E_{\hat{0} \mu} k^{\mu}\right)\right|_{\chi}}{\left.\left(E_{\hat{0} \mu} k^{\mu}\right)\right|_{o}}
$$


where we used $\nu \propto 1 / a$. Quantities evaluated at the observer have a subscript o, while other quantities are assumed to be evaluated at the emitter (we suppress a subscript e for convenience). Choosing ${ }^{1} a_{o}=1$ and given ${ }^{2}$

$$
\left.\left(E_{\hat{0} \mu} k^{\mu}\right)\right|_{o}=1
$$

then

$$
1+z=\frac{E_{\hat{0} \mu} k^{\mu}}{a} .
$$

From Eq. (19), $\bar{a}$ is the scale factor in redshift-space. Then $\bar{a}=1 /(1+z)$. From Eqs. (19), 25) and (26), we get

$$
1=\frac{1+\left(E_{\hat{0} \mu} k^{\mu}\right)^{(1)}+\frac{1}{2}\left(E_{\hat{0} \mu} k^{\mu}\right)^{(2)}}{1+\Delta \ln a^{(1)}+\frac{1}{2} \Delta \ln a^{(2)}},
$$

where

$$
\left(E_{\hat{0} \mu} k^{\mu}\right)^{(0)}=1
$$

Then from Eq. 30 we can find $\Delta \ln a^{(1)}$ and $\Delta \ln a^{(2)}$, such that ${ }^{3}$

$$
\begin{aligned}
\Delta \ln a^{(1)} & =\left(E_{\hat{0} \mu} k^{\mu}\right)^{(1)}=E_{\hat{0} \mu}^{(1)} k^{\mu(0)}+E_{\hat{0} \mu}^{(0)} k^{\mu(1)}=-E_{\hat{0} 0}^{(1)}+n^{i} E_{\hat{0} i}^{(1)}-\delta \nu^{(1)}, \\
\Delta \ln a^{(2)} & =\left(E_{\hat{0} \mu} k^{\mu}\right)^{(2)}=2 E_{\hat{0} \mu}^{(1)} k^{\mu(1)}+E_{\hat{0} \mu}^{(2)} k^{\mu(0)}+E_{\hat{0} \mu}^{(0)} k^{\mu(2)}+2 \delta \chi^{(1)} E_{\hat{0} \mu}^{(0)}\left(\frac{\mathrm{d} k^{\mu}}{\mathrm{d} \bar{\chi}}\right)^{(1)}+2 \delta \chi^{(1)} k^{\mu(0)}\left(\frac{\mathrm{d} E_{\hat{0} \mu}}{\mathrm{d} \bar{\chi}}\right)^{(1)} \\
& +2 k^{\mu(0)}\left(\frac{\partial E_{\hat{0} \mu}}{\partial \bar{x}^{\nu}}\right)^{(1)} \delta x^{\nu(1)} \\
& =2 E_{\hat{0} \mu}^{(1)} k^{\mu(1)}+E_{\hat{0} \mu}^{(2)} k^{\mu(0)}+E_{\hat{0} \mu}^{(0)} k^{\mu(2)}+2 k^{\mu(0)}\left(\frac{\partial E_{\hat{0} \mu}}{\partial \bar{x}^{\nu}}\right)^{(1)} \delta x^{\nu(1)}+2 \delta \chi^{(1)} \frac{\mathrm{d}}{\mathrm{d} \bar{\chi}} \Delta \ln a^{(1)} \\
& =2 E_{\hat{0} 0}^{(1)} \delta \nu^{(1)}+2 E_{\hat{0} i}^{(1)} \delta n^{i(1)}-\delta \nu^{(2)}-E_{\hat{0} 0}^{(2)}+n^{i} E_{\hat{0} i}^{(2)}+2\left[-\left(\frac{\partial E_{\hat{0} 0}}{\partial \bar{x}^{\nu}}\right)^{(1)}+n^{i}\left(\frac{\partial E_{\hat{0} i}}{\partial \bar{x}^{\nu}}\right)^{(1)}\right] \delta x^{\nu(1)} \\
& +2 \delta \chi^{(1)} \frac{\mathrm{d}}{\mathrm{d} \bar{\chi}} \Delta \ln a^{(1)} .
\end{aligned}
$$

Using Eqs. 20 and (32) at first order, and Eqs. 21) and (33) at second order we obtain

$$
\begin{aligned}
& \delta \chi^{(1)}=\delta x^{0(1)}-\frac{\Delta \ln a^{(1)}}{\mathcal{H}}=\delta x^{0(1)}-\Delta x^{0(1)} \\
& \delta \chi^{(2)}=-\frac{1}{\mathcal{H}} \Delta \ln a^{(2)}+\frac{\left(\mathcal{H}^{\prime}+\mathcal{H}^{2}\right)}{\mathcal{H}^{3}}\left(\Delta \ln a^{(1)}\right)^{2}-\frac{2}{\mathcal{H}} \delta \nu^{(1)} \Delta \ln a^{(1)}+2 \delta \nu^{(1)} \delta x^{0(1)}+\delta x^{0(2)} .
\end{aligned}
$$

Given $\Delta \ln a^{(1)}$ from Eq. 32 , and $\Delta \ln a^{(2)}$ from Eq. 33 , it is useful to rewrite Eqs. 20, and 21, as

$$
\begin{aligned}
& \Delta x^{0(1)}=\frac{\Delta \ln a^{(1)}}{\mathcal{H}}, \\
& \Delta x^{0(2)}=\frac{1}{\mathcal{H}} \Delta \ln a^{(2)}-\frac{\left(\mathcal{H}^{\prime}+\mathcal{H}^{2}\right)}{\mathcal{H}^{3}}\left(\Delta \ln a^{(1)}\right)^{2} .
\end{aligned}
$$

\footnotetext{
${ }^{1}$ Another possibility is the generalization $a_{o} \neq \bar{a}_{o}=a\left(\bar{x}^{0}\right)=1$. Then $a_{o}=\left.a(\eta)\right|_{o}=1+\Delta \ln a_{o}^{(1)}+\Delta \ln a_{o}^{(2)} / 2$, and we should add $-\Delta \ln a_{o}^{(1)}$ to the right side of Eq. 32 and $-\Delta \ln a_{o}^{(2)} / 2$ to the right side of Eq. 33. In order to obtain $\Delta \ln a_{o}^{(1)}$ and $\Delta \ln a_{o}^{(2)} / 2$ we can follow the prescription used in [14] (which computes $a_{o}$ only at first order). In our case, i.e. when we assume $a_{o}=1$, by construction we automatically have $\Delta \ln a_{o}^{(1)}=\Delta \ln a_{o}^{(2)} / 2=0$.

${ }^{2}$ From Eq. 99, for $\bar{\chi}=0$ we have $p_{\hat{0} o}=\left.\left(\Lambda_{\hat{0} \mu} p^{\mu}\right)\right|_{o}=\nu_{o}, p_{\hat{a} o}=\left.\left(\Lambda_{\hat{a} \mu} p^{\mu}\right)\right|_{o}=n_{\hat{a}} \nu_{o}$.

3 In Eq. 33 we have used $k^{\nu(0)}\left[\partial E_{\hat{0} \mu} / \partial \bar{x}^{\nu}\right]^{(1)}=\left(\mathrm{d} \bar{x}^{\nu} / \mathrm{d} \bar{\chi}\right)\left[\partial E_{\hat{0} \mu} / \partial \bar{x}^{\nu}\right]^{(1)}=\left[\mathrm{d} E_{\hat{0} \mu} / \mathrm{d} \bar{\chi}\right]^{(1)}$.
} 


\section{Number density}

The physical number density of galaxies $n_{g}$ as a function of physical comoving coordinates $x^{\mu}$ is defined by the observed number of galaxies contained within a volume $\overline{\mathcal{V}}$ :

$$
\mathcal{N}=\int_{\overline{\mathcal{V}}} \sqrt{-g\left(x^{\alpha}\right)} n_{g}\left(x^{\alpha}\right) \varepsilon_{\mu \nu \rho \sigma} u^{\mu}\left(x^{\alpha}\right) \frac{\partial x^{\nu}}{\partial \bar{x}^{1}} \frac{\partial x^{\rho}}{\partial \bar{x}^{2}} \frac{\partial x^{\sigma}}{\partial \bar{x}^{3}} \mathrm{~d}^{3} \overline{\mathbf{x}}
$$

where $\varepsilon_{\mu \nu \rho \sigma}$ is the Levi-Civita tensor, and $u^{\mu}$ is the four velocity vector as a function of comoving location. In the redshift frame

$$
\mathcal{N}=\int_{\overline{\mathcal{V}}} \bar{a}^{3}\left(\bar{x}^{0}\right) n_{g}\left(\bar{x}^{0}, \overline{\mathbf{x}}\right) \mathrm{d}^{3} \overline{\mathbf{x}}
$$

so that

$$
a\left(\bar{x}^{0}\right)^{3} n_{g}\left(\bar{x}^{0}, \overline{\mathbf{x}}\right)=\sqrt{-g\left(x^{\alpha}\right)} n_{g}\left(x^{\alpha}\right) \varepsilon_{\mu \nu \rho \sigma} u^{\mu}\left(x^{\alpha}\right) \frac{\partial x^{\nu}}{\partial \bar{x}^{1}} \frac{\partial x^{\rho}}{\partial \bar{x}^{2}} \frac{\partial x^{\sigma}}{\partial \bar{x}^{3}} .
$$

Using the cosmic rulers defined in the previous section we expand all quantities on the left side of Eq. 40 in the observed coordinates, i.e. in the redshift frame. Using Eq. 23,

$$
\sqrt{-\hat{g}\left(x^{\alpha}\right)} a^{3}\left(x^{0}\right) n_{g}\left(x^{\alpha}\right) \varepsilon_{\mu \nu \rho \sigma} E_{\hat{0}}^{\mu}\left(x^{\alpha}\right) \frac{\partial x^{\nu}}{\partial \bar{x}^{1}} \frac{\partial x^{\rho}}{\partial \bar{x}^{2}} \frac{\partial x^{\sigma}}{\partial \bar{x}^{3}}
$$

where $\hat{g}$ is the determinant of the comoving metric $\hat{g}_{\mu \nu}=g_{\mu \nu} / a^{2}$. We separate Eq. 41, into three parts:

$$
\text { (1) } \sqrt{-\hat{g}\left(x^{\alpha}\right)}, \quad \text { (2) } \varepsilon_{\mu \nu \rho \sigma} E_{\hat{0}}^{\mu}\left(x^{\alpha}\right) \frac{\partial x^{\nu}}{\partial \bar{x}^{1}} \frac{\partial x^{\rho}}{\partial \bar{x}^{2}} \frac{\partial x^{\sigma}}{\partial \bar{x}^{3}} \quad \text { and } \quad \text { (3) } a^{3}\left(x^{0}\right) n_{g}\left(x^{\alpha}\right) \text {. }
$$

Then $^{4}$ :

(1) Splitting $\sqrt{-\hat{g}\left(x^{\alpha}\right)}$ as

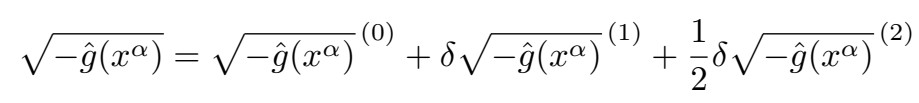

we have

$$
\begin{aligned}
& \delta{\sqrt{-\hat{g}\left(x^{\alpha}\right)}}^{(1)}=\frac{1}{2}{\sqrt{-\hat{g}\left(x^{\alpha}\right)}}^{(0)} \hat{g}_{\mu}^{\mu(1)}\left(x^{\alpha}\right), \\
& \delta{\sqrt{-\hat{g}\left(x^{\alpha}\right)}}^{(2)}=\frac{1}{2}{\sqrt{-\hat{g}\left(x^{\alpha}\right)}}^{(0)}\left(\frac{1}{2} \hat{g}_{\mu}^{\mu(1)} \hat{g}_{\nu}^{\nu(1)}+\hat{g}_{\mu}^{\mu(2)}-\hat{g}_{\mu}^{\nu(1)} \hat{g}_{\nu}^{\mu(1)}\right)\left(x^{\alpha}\right) .
\end{aligned}
$$

Here $\hat{g}_{\nu}^{\mu(n)}=\hat{g}^{\mu \sigma(0)} \hat{g}_{\sigma \nu}^{(n)}$ and $\hat{g}_{\mu \nu}=\hat{g}_{\mu \nu}^{(0)}+\hat{g}_{\mu \nu}^{(1)}+\hat{g}_{\mu \nu}^{(2)} / 2$.

Mapping all these terms from real- to redshift-space, we find

$$
\begin{aligned}
{\sqrt{-\hat{g}\left(x^{\alpha}\right)}}^{(0)} & =1 \\
\delta{\sqrt{-\hat{g}\left(x^{\alpha}\right)}}^{(1)} & =\delta{\sqrt{-\hat{g}\left(\bar{x}^{\alpha}\right)}}^{(1)}+\left(\frac{\partial}{\partial x^{\nu}} \delta{\left.\sqrt{-\hat{g}\left(\bar{x}^{\alpha}\right)}\right)^{(1)} \Delta x^{\nu(1)}}=\frac{1}{2} \hat{g}_{\mu}^{\mu(1)}\left(\bar{x}^{\alpha}\right)+\frac{1}{2}\left(\frac{\partial \hat{g}_{\mu}^{\mu}}{\partial \bar{x}^{\nu}}\right)^{(1)}\left(\bar{x}^{\alpha}\right) \Delta x^{\nu(1)}\right. \\
\delta{\sqrt{-\hat{g}\left(x^{\alpha}\right)}}^{(2)} & =\delta{\sqrt{-\hat{g}\left(\bar{x}^{\alpha}\right)}}^{(2)}
\end{aligned}
$$

\footnotetext{
${ }^{4}$ For a rank two tensor $\mathbb{M}$,

$$
M=\operatorname{det}(\mathbb{M})=M^{(0)}+M^{(1)}+M^{(2)} / 2, \quad \text { where } \quad M^{(0)}=\operatorname{det}\left(\mathbb{M}^{(0)}\right), \quad M^{(1)}=M^{(0)} \operatorname{Tr}\left[\mathbb{M}^{(0)-1} \mathbb{M}^{(1)}\right],
$$$$
M^{(2)}=M^{(0)}\left\{\left[M^{(1)} / M^{(0)}\right]^{2}-\operatorname{Tr}\left[\left(\mathbb{M}^{(0)-1} \mathbb{M}^{(1)}\right)\left(\mathbb{M}^{(0)-1} \mathbb{M}^{(1)}\right)\right]+\operatorname{Tr}\left[\mathbb{M}^{(0)-1} \mathbb{M}^{(2)}\right]\right\} \text {. }
$$ 
Rewriting

$$
{\sqrt{-\hat{g}\left(x^{\alpha}\right)}}^{2}+\Delta{\sqrt{-\hat{g}\left(\bar{x}^{\alpha}\right)}}^{(1)}+\frac{1}{2} \Delta{\sqrt{-\hat{g}\left(\bar{x}^{\alpha}\right)}}^{(2)}
$$

we find

$$
\begin{aligned}
& \Delta{\sqrt{-\hat{g}\left(\bar{x}^{\alpha}\right)}}^{(1)}=\frac{1}{2} \hat{g}_{\mu}^{\mu(1)}\left(\bar{x}^{\alpha}\right) \\
& \Delta{\sqrt{-\hat{g}\left(\bar{x}^{\alpha}\right)}}^{(2)}=\frac{1}{4} \hat{g}_{\mu}^{\mu(1)}\left(\bar{x}^{\alpha}\right) \hat{g}_{\nu}^{\nu(1)}\left(\bar{x}^{\alpha}\right)+\frac{1}{2} \hat{g}_{\mu}^{\mu(2)}\left(\bar{x}^{\alpha}\right)-\frac{1}{2} \hat{g}_{\mu}^{\nu(1)}\left(\bar{x}^{\alpha}\right) \hat{g}_{\nu}^{\mu(1)}\left(\bar{x}^{\alpha}\right)+\left(\frac{\partial \hat{g}_{\mu}^{\mu}}{\partial \bar{x}^{\nu}}\right)^{(1)}\left(\bar{x}^{\alpha}\right) \Delta x^{\nu(1)} .(5
\end{aligned}
$$

(2) We write Eq. 42 as

$$
\varepsilon_{\mu \nu \rho \sigma} E_{\hat{0}}^{\mu}\left(x^{\alpha}\right) \frac{\partial x^{\nu}}{\partial \bar{x}^{1}} \frac{\partial x^{\rho}}{\partial \bar{x}^{2}} \frac{\partial x^{\sigma}}{\partial \bar{x}^{3}}=E_{\hat{0}}^{0}\left(x^{\alpha}\right)\left|\frac{\partial \mathbf{x}}{\partial \overline{\mathbf{x}}}\right|+E_{\hat{0}}^{i}\left(x^{\alpha}\right) \Sigma_{i}
$$

where

$$
\left|\frac{\partial \mathbf{x}}{\partial \overline{\mathbf{x}}}\right|=\operatorname{det}\left(\frac{\partial x^{i}}{\partial \bar{x}^{j}}\right) \quad \text { and } \quad \Sigma_{i}=\epsilon_{i j k}\left(-\frac{\partial x^{0}}{\partial \bar{x}^{1}} \frac{\partial x^{j}}{\partial \bar{x}^{2}} \frac{\partial x^{k}}{\partial \bar{x}^{3}}+\frac{\partial x^{j}}{\partial \bar{x}^{1}} \frac{\partial x^{0}}{\partial \bar{x}^{2}} \frac{\partial x^{k}}{\partial \bar{x}^{3}}-\frac{\partial x^{j}}{\partial \bar{x}^{1}} \frac{\partial x^{k}}{\partial \bar{x}^{2}} \frac{\partial x^{0}}{\partial \bar{x}^{3}}\right) .
$$

The first term on the right of Eq. 51] is

$$
E_{\hat{0}}^{0}\left(x^{\alpha}\right)\left|\frac{\partial \mathbf{x}}{\partial \overline{\mathbf{x}}}\right|=\left[E_{\hat{0}}^{0(0)}\left(x^{\alpha}\right)+E_{\hat{0}}^{0(1)}\left(x^{\alpha}\right)+\frac{1}{2} E_{\hat{0}}^{0(2)}\left(x^{\alpha}\right)\right]\left[\left|\frac{\partial \mathbf{x}}{\partial \overline{\mathbf{x}}}\right|^{(0)}+\left|\frac{\partial \mathbf{x}}{\partial \overline{\mathbf{x}}}\right|^{(1)}+\frac{1}{2}\left|\frac{\partial \mathbf{x}}{\partial \overline{\mathbf{x}}}\right|^{(2)}\right]
$$

where

$$
\begin{aligned}
E_{\hat{0}}^{0(0)} & =1 \quad E_{\hat{0}}^{0(1)}\left(x^{\alpha}\right)=E_{\hat{0}}^{0(1)}\left(\bar{x}^{\alpha}\right)+\left(\frac{\partial E_{\hat{0}}^{0}}{\partial \bar{x}^{\mu}}\right)^{(1)}\left(\bar{x}^{\alpha}\right) \Delta x^{\mu(1)}, \\
\left|\frac{\partial \mathbf{x}}{\partial \overline{\mathbf{x}}}\right|^{(0)} & =1, \quad\left|\frac{\partial \mathbf{x}}{\partial \overline{\mathbf{x}}}\right|^{(1)}=\left(\frac{\partial \Delta x^{i}}{\partial \bar{x}^{i}}\right)^{(1)}, \\
\left|\frac{\partial \mathbf{x}}{\partial \overline{\mathbf{x}}}\right|^{(2)} & =\left(\frac{\partial \Delta x^{i}}{\partial \bar{x}^{i}}\right)^{(1)}\left(\frac{\partial \Delta x^{j}}{\partial \bar{x}^{j}}\right)^{(1)}-\left(\frac{\partial \Delta x^{i}}{\partial \bar{x}^{j}}\right)^{(1)}\left(\frac{\partial \Delta x^{j}}{\partial \bar{x}^{i}}\right)^{(1)}+\left(\frac{\partial \Delta x^{i}}{\partial \bar{x}^{i}}\right)^{(2)} .
\end{aligned}
$$

The second term on the right of Eq. 51 is

$$
E_{\hat{0}}^{i}\left(x^{\alpha}\right) \Sigma_{i}=\left[E_{\hat{0}}^{i(0)}\left(x^{\alpha}\right)+E_{\hat{0}}^{i(1)}\left(x^{\alpha}\right)+\frac{1}{2} E_{\hat{0}}^{i(2)}\left(x^{\alpha}\right)\right]\left[\Sigma_{i}^{(0)}+\Sigma_{i}^{(1)}+\frac{1}{2} \Sigma_{i}^{(2)}\right],
$$

where

$$
\begin{aligned}
& E_{\hat{0}}^{i(0)}=0 \quad E_{\hat{0}}^{i(1)}\left(x^{\alpha}\right)=E_{\hat{0}}^{i(1)}\left(\bar{x}^{\alpha}\right)+\left(\frac{\partial E_{\hat{0}}^{i}}{\partial \bar{x}^{\mu}}\right)^{(1)}\left(\bar{x}^{\alpha}\right) \Delta x^{\mu(1)} \\
& \Sigma_{i}^{(0)}=n_{i}, \quad \Sigma_{i}^{(1)}=-\left(\frac{\partial \Delta x^{0}}{\partial \bar{x}^{i}}\right)^{(1)}+\epsilon_{i j r} \epsilon^{p q r} n_{p}\left(\frac{\partial \Delta x^{j}}{\partial \bar{x}^{q}}\right)^{(1)} .
\end{aligned}
$$

Here we do not compute $\Sigma_{i}^{(2)}$ because $E_{\hat{0}}^{i(0)}=0$.

(3) From Eq. 19,

$$
a^{3}=\bar{a}^{3}\left[1+3 \Delta \ln a^{(1)}+3\left(\Delta \ln a^{(1)}\right)^{2}+\frac{3}{2} \Delta \ln a^{(2)}\right]
$$

Writing $n_{g}\left(x^{\alpha}\right)=n_{g}^{(0)}\left(x^{0}\right)+n_{g}^{(1)}\left(x^{\alpha}\right)+n_{g}^{(2)}\left(x^{\alpha}\right) / 2$, we have

$$
n_{g}^{(0)}\left(x^{0}\right)=n_{g}^{(0)}\left(\bar{x}^{0}+\Delta x^{0}\right)=\bar{n}_{g}\left(\bar{x}^{0}\right)+\frac{\partial \bar{n}_{g}}{\partial \bar{x}^{0}} \Delta x^{0(1)}+\frac{1}{2} \frac{\partial^{2} \bar{n}_{g}}{\partial \bar{x}^{0^{2}}}\left(\Delta x^{0(1)}\right)^{2}+\frac{1}{2} \frac{\partial \bar{n}_{g}}{\partial \bar{x}^{0}} \Delta x^{0(2)}
$$


where $n_{g}^{(0)}\left(\bar{x}^{0}\right)=\bar{n}_{g}\left(\bar{x}^{0}\right)$ and

$$
n_{g}^{(1)}\left(x^{\alpha}\right)=n_{g}^{(1)}\left(\bar{x}^{\alpha}+\Delta x^{\alpha(1)}\right)=n_{g}^{(1)}\left(\bar{x}^{\alpha}\right)+\left(\frac{\partial n_{g}}{\partial \bar{x}^{\alpha}}\right)^{(1)} \Delta x^{\alpha(1)}, \quad \frac{1}{2} n_{g}^{(2)}\left(x^{\alpha}\right)=\frac{1}{2} n_{g}^{(2)}\left(\bar{x}^{\alpha}\right) .
$$

Defining $\delta_{g}^{(n)}=n_{g}^{(n)}\left(\bar{x}^{\alpha}\right) / \bar{n}_{g}\left(\bar{x}^{0}\right)$ and considering Eqs. 36 and 37), we have

$$
\begin{aligned}
n_{g}\left(x^{\alpha}\right) & =\bar{n}_{g}\left\{1+\frac{\mathrm{d} \ln \bar{n}_{g}}{\mathrm{~d} \ln \bar{a}} \Delta \ln a^{(1)}+\delta_{g}^{(1)}+\left(\frac{\partial \delta_{g}}{\partial \bar{x}^{\mu}}\right)^{(1)} \Delta x^{\mu(1)}+\frac{\mathrm{d} \ln \bar{n}_{g}}{\mathrm{~d} \ln \bar{a}} \Delta \ln a^{(1)} \delta_{g}^{(1)}\right. \\
& \left.+\frac{1}{2}\left[-\frac{\mathrm{d} \ln \bar{n}_{g}}{\mathrm{~d} \ln \bar{a}}+\left(\frac{\mathrm{d} \ln \bar{n}_{g}}{\mathrm{~d} \ln \bar{a}}\right)^{2}+\frac{\mathrm{d}^{2} \ln \bar{n}_{g}}{\mathrm{~d} \ln \bar{a}^{2}}\right]\left(\Delta \ln a^{(1)}\right)^{2}+\frac{1}{2} \frac{\mathrm{d} \ln \bar{n}_{g}}{\mathrm{~d} \ln \bar{a}} \Delta \ln a^{(2)}+\frac{1}{2} \delta_{g}^{(2)}\right\} .
\end{aligned}
$$

At this point, it is useful to define the parallel and perpendicular projection operators to the observed line-of-sight direction (see also [5, 7). For any spatial vectors and tensors:

$$
A_{\|}=n^{i} n^{j} A_{i j}, \quad B_{\perp}^{i}=\mathcal{P}^{i j} B_{j}=B^{i}-n^{i} B_{\|}, \quad A_{\perp}=\mathcal{P}^{i j} A_{i j}, \quad \mathcal{P}_{j}^{i}=\delta_{j}^{i}-n^{i} n_{j} .
$$

The directional derivatives are defined as

$$
\partial_{\|}=n^{i} \frac{\partial}{\partial \bar{x}^{i}}, \quad \partial_{\|}^{2}=\partial_{\|} \partial_{\|}, \quad \partial_{\perp i}=\mathcal{P}_{i}^{j} \partial_{j}=\frac{\partial}{\partial \bar{x}^{i}}-n_{i} \partial_{\|},
$$

and we have

$$
\begin{array}{rc}
\frac{\partial n^{j}}{\partial \bar{x}^{i}}=\frac{1}{\bar{\chi}} \mathcal{P}_{i}^{j}, \quad n^{i} B_{\perp i}=0, & n^{i} \partial_{\perp i}=0 \\
\frac{\mathrm{d}}{\mathrm{d} \bar{\chi}} \partial_{\perp}^{i}=\partial_{\perp}^{i} \frac{\mathrm{d}}{\mathrm{d} \bar{\chi}}-\frac{1}{\bar{\chi}} \partial_{\perp}^{i}, & \partial_{\perp i}\left(n^{k} \mathcal{P}_{j}^{i}\right)=\frac{1}{\bar{\chi}}\left(\delta_{j}^{k}-3 n^{k} n_{j}\right) \\
\frac{\partial B^{i}}{\partial \bar{x}^{j}}=n^{i} n_{j} \partial_{\|} B_{\|}+n^{i} \partial_{\perp j} B_{\|}+\partial_{\perp j} B_{\perp}^{i}+n_{j} \partial_{\|} B_{\perp}^{i}+\frac{1}{\bar{\chi}} \mathcal{P}_{j}^{i} B_{\|} \\
\text {and } \\
\nabla_{\perp}^{2}=\partial_{\perp i} \partial_{\perp}^{i}=\delta^{i j} \frac{\partial}{\partial \bar{x}^{i}} \frac{\partial}{\partial \bar{x}^{j}}-\partial_{\|}^{2}-\frac{2}{\bar{\chi}} \partial_{\|} \cdot
\end{array}
$$

We also have

$$
\begin{aligned}
\operatorname{Tr}[\bar{\partial} B] & =\frac{\partial B^{i}}{\partial \bar{x}^{i}}=\partial_{\perp i} B_{\perp}^{i}+\partial_{\|} B_{\|}+\frac{2}{\bar{\chi}} B_{\|} \\
\operatorname{Tr}\left[(\bar{\partial} B)^{2}\right] & =\left(\frac{\partial B^{i}}{\partial \bar{x}^{j}}\right)\left(\frac{\partial B^{j}}{\partial \bar{x}^{i}}\right)=\left(\partial_{\|} B_{\|}\right)\left(\partial_{\|} B_{\|}\right)+\left(\partial_{\perp j} B_{\perp}^{i}\right)\left(\partial_{\perp i} B_{\perp}^{j}\right)-\frac{2}{\bar{\chi}} B_{\perp i} \partial_{\|} B_{\perp}^{i}+\frac{2}{\bar{\chi}} B_{\|} \partial_{\perp i} B_{\perp}^{i} \\
& +2\left(\partial_{\|} B_{\perp}^{i}\right)\left(\partial_{\perp i} B_{\|}\right)+\frac{2}{\bar{\chi}^{2}} B_{\|} B_{\|} .
\end{aligned}
$$

Using Eq. 64, we find $\Delta x^{i(1)}=n^{i} \Delta x_{\|}^{(1)}+\mathcal{P}_{j}^{i} \Delta x^{j(1)}=n^{i} \Delta x_{\|}^{(1)}+\Delta x_{\perp}^{i(1)}$ where

$$
\begin{aligned}
\Delta x_{\|}^{(1)} & =\delta \chi^{(1)}+\delta x_{\|}^{(1)}=\delta x^{0(1)}-\frac{\Delta \ln a^{(1)}}{\mathcal{H}}+\delta x_{\|}^{(1)}=\delta x^{0(1)}+\delta x_{\|}^{(1)}-\Delta x^{0(1)}, \\
\Delta x_{\perp}^{i(1)} & =\delta x_{\perp}^{i(1)} .
\end{aligned}
$$

For $\Delta x^{i(2)}=n^{i} \Delta x_{\|}^{(2)}+\mathcal{P}_{j}^{i} \Delta x^{j(2)}=n^{i} \Delta x_{\|}^{(2)}+\Delta x_{\perp}^{i(2)}$ we have

$$
\begin{aligned}
\Delta x_{\|}^{(2)}= & \delta \chi^{(2)}+2 \delta n_{\|}^{(1)} \delta \chi^{(1)}+\delta x_{\|}^{(2)}=-\frac{1}{\mathcal{H}} \Delta \ln a^{(2)}+\frac{\left(\mathcal{H}^{\prime}+\mathcal{H}^{2}\right)}{\mathcal{H}^{3}}\left(\Delta \ln a^{(1)}\right)^{2}-\frac{2}{\mathcal{H}} \delta \nu^{(1)} \Delta \ln a^{(1)} \\
& +2 \delta \nu^{(1)} \delta x^{0(1)}+\delta x^{0(2)}+2 \delta n_{\|}^{(1)} \delta x^{0(1)}-\frac{2}{\mathcal{H}} \delta n_{\|}^{(1)} \Delta \ln a^{(1)}+\delta x_{\|}^{(2)} \\
= & \delta x^{0(2)}+\delta x_{\|}^{(2)}-\Delta x^{0(2)}+2\left(\delta \nu^{(1)}+\delta n_{\|}^{(1)}\right) \delta \chi^{(1)} \\
\Delta x_{\perp}^{i(2)}= & 2 \delta n_{\perp}^{i(1)} \delta \chi^{(1)}+\delta x_{\perp}^{i(2)}=2 \delta n_{\perp}^{i(1)} \delta x^{0(1)}-\frac{2}{\mathcal{H}} \delta n_{\perp}^{i(1)} \Delta \ln a^{(1)}+\delta x_{\perp}^{i(2)} .
\end{aligned}
$$


We define the parallel and perpendicular parts of the tetrad in the comoving frame ${ }^{5}$ :

$$
\begin{aligned}
E_{\hat{\alpha}}^{i} & =n^{i} E_{\hat{\alpha}}^{\|}+E_{\hat{\alpha}}^{\perp i}, \quad \text { where } \quad E_{\hat{\alpha}}^{\|}=n_{i} E_{\hat{\alpha}}^{i} \quad \text { and } \quad E_{\hat{\alpha}}^{\perp i}=\mathcal{P}_{j}^{i} E_{\hat{\alpha}}^{j}, \\
E_{\hat{\alpha} i} & =n_{i} E_{\hat{\alpha} \|}+E_{\hat{\alpha} \perp i}, \quad \text { where } \quad E_{\hat{\alpha} \|}=n^{i} E_{\hat{\alpha} i} \quad \text { and } \quad E_{\hat{\alpha} \perp i}=\mathcal{P}_{i}^{j} E_{\hat{\alpha} j} .
\end{aligned}
$$

Taking into account Eqs. (64), (65), (66) and $(67)$ and observing that $\epsilon_{i j r} \epsilon^{p q r}=\left(\delta_{i}^{p} \delta_{j}^{q}-\delta_{j}^{p} \delta_{i}^{q}\right)$, we obtain the observed fractional number over-density

$$
\Delta_{g}=\frac{n_{g}\left(\bar{x}^{0}, \overline{\mathbf{x}}\right)-\bar{n}_{g}\left(\bar{x}^{0}\right)}{\bar{n}_{g}\left(\bar{x}^{0}\right)}=\Delta_{g}^{(1)}+\frac{1}{2} \Delta_{g}^{(2)}
$$

where

$$
\begin{aligned}
& \Delta_{g}^{(1)}=\delta_{g}^{(1)}+\frac{1}{2} \hat{g}_{\mu}^{\mu(1)}+b_{e} \Delta \ln a^{(1)}+\partial_{\|} \Delta x_{\|}^{(1)}+\frac{2}{\bar{\chi}} \Delta x_{\|}^{(1)}-2 \kappa^{(1)}+E_{\hat{0}}^{0(1)}+E_{\hat{0}}^{\|(1)}, \\
& \Delta_{g}^{(2)}=\delta_{g}^{(2)}+\frac{1}{2} \hat{g}_{\mu}^{\mu(2)}+b_{e} \Delta \ln a^{(2)}+\partial_{\|} \Delta x_{\|}^{(2)}+\frac{2}{\bar{\chi}} \Delta x_{\|}^{(2)}-2 \kappa^{(2)}+E_{\hat{0}}^{0(2)}+E_{\hat{0}}^{\|(2)} \\
& +\frac{1}{4} \hat{g}_{\mu}^{\mu(1)} \hat{g}_{\nu}^{\nu(1)}-\frac{1}{2} \hat{g}_{\mu}^{\nu(1)} \hat{g}_{\nu}^{\mu(1)}+\frac{1}{\mathcal{H}} \hat{g}_{\mu}^{\mu(1) \prime} \Delta \ln a^{(1)}+\left(\partial_{\|} \hat{g}_{\mu}^{\mu(1)}\right) \Delta x_{\|}^{(1)}+\left(\partial_{\perp i} \hat{g}_{\mu}^{\mu(1)}\right) \Delta x_{\perp}^{i(1)} \\
& +\left(-b_{e}+b_{e}^{2}+\frac{\mathrm{d} \ln b_{e}}{\mathrm{~d} \ln \bar{a}}\right)\left(\Delta \ln a^{(1)}\right)^{2}+2 b_{e} \Delta \ln a^{(1)} \delta_{g}^{(1)}+\frac{2}{\mathcal{H}} \delta_{g}^{(1) \prime} \Delta \ln a^{(1)}+2 \partial_{\|} \delta_{g}^{(1)} \Delta x_{\|}^{(1)} \\
& +2 \partial_{\perp}^{i} \delta_{g}^{(1)} \Delta x_{\perp i}^{(1)}+4\left(\kappa^{(1)}\right)^{2}+\frac{2}{\bar{\chi}^{2}}\left(\Delta x_{\|}^{(1)}\right)^{2}-4 \kappa^{(1)} \partial_{\|} \Delta x_{\|}^{(1)}-\frac{4}{\bar{\chi}} \kappa^{(1)} \Delta x_{\|}^{(1)}+\frac{4}{\bar{\chi}} \Delta x_{\|}^{(1)} \partial_{\|} \Delta x_{\|}^{(1)} \\
& -\left(\partial_{\perp j} \Delta x_{\perp}^{i(1)}\right)\left(\partial_{\perp i} \Delta x_{\perp}^{j(1)}\right)+\frac{2}{\bar{\chi}} \Delta x_{\perp i}^{(1)} \partial_{\|} \Delta x_{\perp}^{i(1)}-2\left(\partial_{\|} \Delta x_{\perp}^{i(1)}\right)\left(\partial_{\perp i} \Delta x_{\|}^{(1)}\right)+\frac{2}{\mathcal{H}} E_{\hat{0}}^{0(1) \prime} \Delta \ln a^{(1)} \\
& +2 \partial_{\|} E_{\hat{0}}^{0(1)} \Delta x_{\|}^{(1)}+2 \partial_{\perp i} E_{\hat{0}}^{0(1)} \Delta x_{\perp}^{i(1)}+2 E_{\hat{0}}^{0(1)} \partial_{\|} \Delta x_{\|}^{(1)}-4 E_{\hat{0}}^{0(1)} \kappa^{(1)}+\frac{4}{\bar{\chi}} E_{\hat{0}}^{0(1)} \Delta x_{\|}^{(1)}+\frac{2}{\mathcal{H}} E_{\hat{0}}^{\|(1) \prime} \Delta \ln a^{(1)} \\
& +2 \partial_{\|} E_{\hat{0}}^{\|(1)} \Delta x_{\|}^{(1)}+2 \partial_{\perp i} E_{\hat{0}}^{\|(1)} \Delta x_{\perp}^{i(1)}-2 E_{\hat{0}}^{\|(1)} \partial_{\|} \Delta x^{0(1)}-2 E_{\hat{0}}^{\perp i(1)} \partial_{\perp i} \Delta x^{0(1)}-4 E_{\hat{0}}^{\|(1)} \kappa^{(1)}+\frac{4}{\bar{\chi}} E_{\hat{0}}^{\|(1)} \Delta x_{\|}^{(1)} \\
& -2 E_{\hat{0}}^{\perp i(1)} \partial_{\perp i} \Delta x_{\|}^{(1)}+b_{e} \hat{g}_{\mu}^{\mu(1)} \Delta \ln a^{(1)}+\hat{g}_{\mu}^{\mu(1)} \delta_{g}^{(1)}+2\left(\frac{1}{2} \hat{g}_{\mu}^{\mu(1)}+b_{e} \Delta \ln a^{(1)}+\delta_{g}^{(1)}\right) \\
& \times\left(\partial_{\|} \Delta x_{\|}^{(1)}-2 \kappa^{(1)}+\frac{2}{\bar{\chi}} \Delta x_{\|}^{(1)}+E_{\hat{0}}^{0(1)}+E_{\hat{0}}^{\|(1)}\right) \\
& =\delta_{g}^{(2)}+\frac{1}{2} \hat{g}_{\mu}^{\mu(2)}+b_{e} \Delta \ln a^{(2)}+\partial_{\|} \Delta x_{\|}^{(2)}+\frac{2}{\bar{\chi}} \Delta x_{\|}^{(2)}-2 \kappa^{(2)}+E_{\hat{0}}^{0(2)}+E_{\hat{0}}^{\|(2)}+\left(\Delta_{g}^{(1)}\right)^{2} \\
& -\frac{1}{2} \hat{g}_{\mu}^{\nu(1)} \hat{g}_{\nu}^{\mu(1)}+\frac{1}{\mathcal{H}} \hat{g}_{\mu}^{\mu(1) \prime} \Delta \ln a^{(1)}+\left(\partial_{\|} \hat{g}_{\mu}^{\mu(1)}\right) \Delta x_{\|}^{(1)}+\left(\partial_{\perp i} \hat{g}_{\mu}^{\mu(1)}\right) \Delta x_{\perp}^{i(1)}+\frac{2}{\mathcal{H}} \delta_{g}^{(1) \prime} \Delta \ln a^{(1)} \\
& +2 \partial_{\|} \delta_{g}^{(1)} \Delta x_{\|}^{(1)}+2 \partial_{\perp}^{i} \delta_{g}^{(1)} \Delta x_{\perp i}^{(1)}+\left(-b_{e}+\frac{\mathrm{d} \ln b_{e}}{\mathrm{~d} \ln \bar{a}}\right)\left(\Delta \ln a^{(1)}\right)^{2}-\frac{2}{\bar{\chi}^{2}}\left(\Delta x_{\|}^{(1)}\right)^{2}+\frac{4}{\bar{\chi}} \kappa^{(1)} \Delta x_{\|}^{(1)} \\
& -\left(\partial_{\perp j} \Delta x_{\perp}^{i(1)}\right)\left(\partial_{\perp i} \Delta x_{\perp}^{j(1)}\right)+\frac{2}{\bar{\chi}} \Delta x_{\perp i}^{(1)} \partial_{\|} \Delta x_{\perp}^{i(1)}-2\left(\partial_{\|} \Delta x_{\perp}^{i(1)}\right)\left(\partial_{\perp i} \Delta x_{\|}^{(1)}\right)+\frac{2}{\mathcal{H}} E_{\hat{0}}^{0(1) \prime} \Delta \ln a^{(1)} \\
& +2 \partial_{\|} E_{\hat{0}}^{0(1)} \Delta x_{\|}^{(1)}+\frac{2}{\mathcal{H}} E_{\hat{0}}^{\|(1) \prime} \Delta \ln a^{(1)}+2 \partial_{\|} E_{\hat{0}}^{\|(1)} \Delta x_{\|}^{(1)}+2 \partial_{\perp i}\left(E_{\hat{0}}^{0(1)}+E_{\hat{0}}^{\|(1)}\right) \Delta x_{\perp}^{i(1)}-2\left(\delta n_{\|}^{(1)}+\delta \nu^{(1)}\right) E_{\hat{0}}^{\|(1)} \\
& -2 E_{\hat{0}}^{\perp i(1)} \partial_{\perp i}\left(\Delta x^{0(1)}+\Delta x_{\|}^{(1)}\right)-\left(\delta_{g}^{(1)}\right)^{2}-\left(\partial_{\|} \Delta x_{\|}^{(1)}\right)^{2}-\left(E_{\hat{0}}^{0(1)}+E_{\hat{0}}^{\|(1)}\right)^{2} .
\end{aligned}
$$

Here

$$
b_{e}=\frac{\mathrm{d} \ln \left(\bar{a}^{3} \bar{n}_{g}\right)}{\mathrm{d} \ln \bar{a}}
$$

\footnotetext{
${ }^{5}$ Note that in general $E_{\hat{\alpha}}^{i}$ is not a 3-space tensor in the index $i$, so that $E_{\hat{0}}^{\|} \neq E_{\hat{0} \|}$ and $E_{\hat{0}}^{\perp i} \neq \delta^{i j} E_{\hat{0} \perp j}$.
} 
is the evolution bias term related to the comoving number density [5]. Note that

$$
\partial_{\|} \Delta x^{\mu(n)}(\bar{\chi}, \mathbf{n})=\partial_{\bar{\chi}} \Delta x^{\mu(n)}
$$

where $\partial_{\bar{\chi}}$ is applied to all terms that are functions of $\bar{x}^{0}=\bar{\eta}(\bar{\chi})$ and $/$ or $\bar{x}^{i}=\bar{x}^{i}(\bar{\chi})$.

\section{E. Weak lensing terms}

In Eq. 777, we introduced the coordinate weak lensing convergence terms at order $n$ :

$$
\kappa^{(n)}=-\frac{1}{2} \partial_{\perp i} \Delta x_{\perp}^{i(n)} .
$$

Then the second-order transverse part of the volume distortion, which appears in Eq. (77), is

$$
-\kappa^{(2)}+2\left(\kappa^{(1)}\right)^{2}-\frac{1}{2}\left(\partial_{\perp j} \Delta x_{\perp}^{i(1)}\right)\left(\partial_{\perp i} \Delta x_{\perp}^{j(1)}\right) .
$$

The coordinate weak lensing shear $\gamma$ and rotation $\vartheta$ do not contribute to the observed number counts at first order but quadratic products do contribute at second order, via the third term above. They are defined by splitting $\partial_{\perp i} \Delta x_{\perp j}^{(1)}$ into its trace, tracefree and antisymmetric parts:

$$
\partial_{\perp i} \Delta x_{\perp j}^{(1)}=-\gamma_{i j}^{(1)}-\mathcal{P}_{i j} \kappa^{(1)}-\vartheta_{i j}^{(1)},
$$

where

$$
\gamma_{i j}^{(1)}=-\partial_{\perp(i} \Delta x_{\perp j)}^{(1)}-\mathcal{P}_{i j} \kappa^{(1)}, \quad \vartheta_{i j}^{(1)}=-\partial_{\perp[i} \Delta x_{\perp j]}^{(1)} .
$$

Then

$$
\left(\partial_{\perp j} \Delta x_{\perp}^{i(1)}\right)\left(\partial_{\perp i} \Delta x_{\perp}^{j(1)}\right)=2\left(\kappa^{(1)}\right)^{2}+2\left|\gamma^{(1)}\right|^{2}-\vartheta_{i j}^{(1)} \vartheta^{i j(1)}
$$

where $2\left|\gamma^{(1)}\right|^{2}=\gamma_{i j}^{(1)} \gamma^{i j(1)}$. Explicit expressions for $\gamma_{i j}^{(1)}$ and $\vartheta_{i j}^{(1)} \vartheta^{i j(1)}$ are given in Eqs. A15] and A16] in a general gauge.

\section{PERTURBED FLAT ROBERTSON-WALKER BACKGROUND IN A GENERAL GAUGE}

The results obtained in the previous section have not yet used the specific form of the metric. Here we assume a spatially flat RW background, perturbed in a general gauge to second order:

$$
\mathrm{d} s^{2}=a(\eta)^{2}\left[-\left(1+2 A^{(1)}+A^{(2)}\right) \mathrm{d} \eta^{2}-2\left(B_{i}^{(1)}+\frac{1}{2} B_{i}^{(2)}\right) \mathrm{d} \eta \mathrm{d} x^{i}+\left(\delta_{i j}+h_{i j}^{(1)}+\frac{1}{2} h_{i j}^{(2)}\right) \mathrm{d} x^{i} \mathrm{~d} x^{j}\right],
$$

where $B^{i(n)}=\partial_{i} B^{(n)}+\hat{B}_{i}^{(n)}$ and $\hat{B}_{i}^{(n)}$ is a solenoidal vector, i.e. $\partial^{i} \hat{B}_{i}^{(n)}=0$. The 3 -tensor is $h_{i j}^{(n)}=2 D^{(n)} \delta_{i j}+F_{i j}^{(n)}$, where $F_{i j}^{(n)}=\left(\partial_{i} \partial_{j}-\delta_{i j} \nabla^{2} / 3\right) F^{(n)}+\partial_{i} \hat{F}_{j}^{(n)}+\partial_{j} \hat{F}_{i}^{(n)}+\hat{h}_{i j}^{(n)}$. Here $D^{(n)}$ and $F^{(n)}$ are scalars and $\hat{F}_{i}^{(n)}$ is a solenoidal vector field, $\partial^{i} \hat{h}_{i j}^{(n)}=\hat{h}_{i}^{i(n)}=0$.

Up to second order, the geodesic equation for $k^{\mu}$ is [21]

$$
\frac{\mathrm{d} k^{\mu}}{\mathrm{d} \bar{\chi}}+\left(\hat{\Gamma}_{\alpha \beta}^{\mu}+\delta x^{\sigma} \frac{\partial \hat{\Gamma}_{\alpha \beta}^{\mu}}{\partial \bar{x}^{\sigma}}\right) k^{\alpha} k^{\beta}=0
$$

where $\hat{\Gamma}_{\alpha \beta}^{\mu}$ are the Christoffel symbols defined using the comoving metric $\hat{g}_{\mu \nu}$. At zeroth order, we obtain Eq. 10. At first order, Eq. (86) yields

$$
\begin{aligned}
\frac{\mathrm{d}}{\mathrm{d} \bar{\chi}}\left(\delta \nu^{(1)}-2 A^{(1)}+B_{\|}^{(1)}\right) & =A^{(1) \prime}-B_{\|}^{(1) \prime}-\frac{1}{2} h_{\|}^{(1) \prime}, \\
\frac{\mathrm{d}}{\mathrm{d} \bar{\chi}}\left(\delta n^{i(1)}+B^{i(1)}+h_{j}^{i(1)} n^{j}\right) & =-\partial^{i} A^{(1)}+\partial^{i} B_{\|}^{(1)}-\frac{1}{\bar{\chi}} B_{\perp}^{i(1)}+\frac{1}{2} \partial^{i} h_{\|}^{(1)}-\frac{1}{\bar{\chi}} \mathcal{P}^{i j} h_{j k}^{(1)} n^{k},
\end{aligned}
$$


in agreement with [7. At second order, we find

$$
\begin{aligned}
& \quad \frac{\mathrm{d}}{\mathrm{d} \bar{\chi}}\left[\delta \nu^{(2)}-2 A^{(2)}+B_{\|}^{(2)}+4 A^{(1)} \delta \nu^{(1)}+2 B_{i}^{(1)} \delta n^{i(1)}\right]=A^{(2) \prime}-B_{\|}^{(2) \prime}-\frac{1}{2} h_{\|}^{(2) \prime}+2 \delta n^{i(1)}\left[\partial_{i}\left(2 A^{(1)}-B_{\|}^{(1)}\right)\right. \\
& \left.-\left(B_{i}^{(1) \prime}+n^{j} h_{i j}^{(1) \prime}\right)+\frac{1}{\bar{\chi}} B_{\perp i}^{(1)}\right]+2\left[\frac{\mathrm{d}}{\mathrm{d} \bar{\chi}}\left(2 A^{(1)^{\prime}}-B_{\|}^{(1)}\right)+\left(A^{(1) \prime \prime}-B_{\|}^{(1) \prime \prime}-\frac{1}{2} h_{\|}^{(1) \prime \prime}\right)\right]\left(\delta x^{0(1)}+\delta x_{\|}^{(1)}\right) \\
& +2 \frac{\mathrm{d}}{\mathrm{d} \bar{\chi}}\left[\frac{\mathrm{d}}{\mathrm{d} \bar{\chi}}\left(2 A^{(1)}-B_{\|}^{(1)}\right)+\left(A^{(1) \prime}-B_{\|}^{(1) \prime}-\frac{1}{2} h_{\|}^{(1) \prime}\right)\right] \delta x_{\|}^{(1)}+2\left\{\partial _ { \perp i } \left[\frac{\mathrm{d}}{\mathrm{d} \bar{\chi}}\left(2 A^{(1)}-B_{\|}^{(1)}\right)\right.\right. \\
& \left.\left.+\left(A^{(1) \prime}-B_{\|}^{(1) \prime}-\frac{1}{2} h_{\|}^{(1) \prime}\right)\right]+\frac{1}{\bar{\chi}}\left[\frac{\mathrm{d} B_{\perp i}^{(1)}}{\mathrm{d} \bar{\chi}}-\partial_{\perp i}\left(2 A^{(1)}-B_{\|}^{(1)}\right)+B_{\perp i}^{(1) \prime}+n^{j} h_{j k}^{(1) \prime} \mathcal{P}_{i}^{k}-\frac{1}{\bar{\chi}} B_{\perp i}^{(1)}\right]\right\} \delta x_{\perp}^{i(1)}, \\
& \frac{\mathrm{d}}{\mathrm{d} \bar{\chi}}\left[\delta n^{i(2)}+B^{i(2)}+h_{j}^{i(2)} n^{j}-2 \delta \nu^{(1)} B^{i(1)}+2 \delta n^{j(1)} h_{j}^{i(1)}\right]=-\partial^{i} A^{(2)}+\partial^{i} B_{\|}^{(2)}-\frac{1}{\bar{\chi}} B_{\perp}^{i(2)}+\frac{1}{2} \partial^{i} h_{\|}^{(2)}-\frac{1}{\bar{\chi}} \mathcal{P}^{i j} h_{j k}^{(2)} n^{k} \\
& +2 \delta \nu^{(1)}\left[2 \partial^{i} A^{(1)}-B^{i(1) \prime}-\partial^{i} B_{\|}^{(1)}+\frac{1}{\bar{\chi}} B_{\perp}^{i(1)}-n^{j} h_{j}^{i(1) \prime}\right]+2 \delta n^{j(1)}\left[\partial^{i} B_{j}^{(1)}-\partial_{j} B^{i(1)}+n^{k} \partial^{i} h_{j k}^{(1)}-n^{k} \partial_{j} h_{k}^{i(1)}\right] \\
& \left.-2\left[\partial^{i}\left(A^{(1)}-B_{\|}^{(1)}-\frac{1}{2} h_{\|}^{(1)}\right)+\frac{\mathrm{d}}{\mathrm{d} \bar{\chi}}\left(B^{i(1)}+n^{j} h_{j}^{i(1)}\right)+\frac{1}{\bar{\chi}}\left(B_{\perp}^{i(1)}+n^{j} h_{j k}^{(1)} \mathcal{P}_{i}^{k}\right)\right]\right]^{\prime}\left(\delta x^{0(1)}+\delta x_{\|}^{(1)}\right) \\
& -2 \frac{\mathrm{d}}{\mathrm{d} \bar{\chi}}\left[\partial^{i}\left(A^{(1)}-B_{\|}^{(1)}-\frac{1}{2} h_{\|}^{(1)}\right)+\frac{\mathrm{d}}{\mathrm{d} \bar{\chi}}\left(B^{i(1)}+n^{j} h_{j}^{i(1)}\right)+\frac{1}{\bar{\chi}}\left(B_{\perp}^{i(1)}+n^{j} h_{j k}^{(1)} \mathcal{P}_{i}^{k}\right)\right] \delta x_{\|}^{(1)} \\
& -2\left\{\partial_{\perp l}\left[\partial^{i}\left(A^{(1)}-B_{\|}^{(1)}-\frac{1}{2} h_{\|}^{(1)}\right)+\frac{\mathrm{d}}{\mathrm{d} \bar{\chi}}\left(B^{i(1)}+n^{j} h_{j}^{i(1)}\right)+\frac{1}{\bar{\chi}}\left(B_{\perp}^{i(1)}+n^{j} h_{j k}^{(1)} \mathcal{P}^{i k}\right)\right]\right. \\
& \left.+\frac{1}{\bar{\chi}} \mathcal{P}_{l}^{j}\left(\partial^{i} B_{j}^{(1)}-\partial_{j} B^{i(1)}-\frac{\mathrm{d}}{\mathrm{d} \bar{\chi}} h_{j}^{i(1)}-n^{k} \partial_{j} h_{k}^{i(1)}+n^{k} \partial^{i} h_{j k}^{(1)}\right)\right\} \delta x_{\perp}^{l(1)} .
\end{aligned}
$$

To solve Eqs. (86), (87), (88) and 89 we require the values of $\delta \nu^{(1)}, \delta \nu^{(2)}, \delta n^{i(1)}$ and $\delta n^{i(2)}$ today. In this case we need all the components of the tetrads $\Lambda_{\mu}^{\hat{\alpha}}$ and $E_{\mu}^{\hat{\alpha}}$ (see Appendix A. Eq. A10) which are defined through the following relations

$$
\begin{aligned}
g^{\mu \nu} \Lambda_{\mu}^{\hat{\alpha}} \Lambda_{\nu}^{\hat{\beta}}=\eta^{\hat{\alpha} \hat{\beta}}, & \eta_{\hat{\alpha} \hat{\beta}} \Lambda_{\mu}^{\hat{\alpha}} \Lambda_{\nu}^{\hat{\beta}}=g_{\mu \nu}, \quad g^{\mu \nu} \Lambda_{\nu}^{\hat{\beta}}=\Lambda^{\hat{\beta} \mu}, \quad \eta_{\hat{\alpha} \hat{\beta}} \Lambda_{\nu}^{\hat{\beta}}=\Lambda_{\hat{\beta} \nu}, \\
\hat{g}^{\mu \nu} E_{\mu}^{\hat{\alpha}} E_{\nu}^{\hat{\beta}}=\eta^{\hat{\alpha} \hat{\beta}}, \quad \eta_{\hat{\alpha} \hat{\beta}} E_{\mu}^{\hat{\alpha}} E_{\nu}^{\hat{\beta}}=\hat{g}_{\mu \nu}, & \hat{g}^{\mu \nu} E_{\nu}^{\hat{\beta}}=E^{\hat{\beta} \mu}, \quad \eta_{\hat{\alpha} \hat{\beta}} E_{\nu}^{\hat{\beta}}=E_{\hat{\beta} \nu},
\end{aligned}
$$

where $\eta_{\hat{\alpha} \hat{\beta}}$ the comoving Minkowski metric. Using Eq. 28) we have, at first order,

$$
\begin{gathered}
\delta \nu_{o}^{(1)}=A_{o}^{(1)}+v_{\| o}^{(1)}-B_{\| o}^{(1)}, \\
\delta n_{o}^{\hat{a}(1)}=-v_{o}^{\hat{a}(1)}-\frac{1}{2} n^{i} h_{i o}^{\hat{a}(1)},
\end{gathered}
$$

and, at second order,

$$
\begin{aligned}
\delta \nu_{o}^{(2)} & =A_{o}^{(2)}+v_{\| o}^{(2)}-B_{\| o}^{(2)}-3\left(A_{o}^{(1)}\right)^{2}-2 v_{\| o}^{(1)} A_{o}^{(1)}+4 B_{\| o}^{(1)} A_{o}^{(1)}-v_{k o}^{(1)} v_{o}^{k(1)}+n^{i} h_{i k o}^{(1)} v_{o}^{k(1)} \\
& +n^{i} h_{i k o}^{(1)} B_{o}^{k(1)}+2 B_{k o}^{(1)} v_{o}^{k(1)}, \\
\delta n_{o}^{\hat{a}(2)} & =-v_{o}^{\hat{a}(2)}-\frac{1}{2} n^{i} h_{i o}^{\hat{a}(2)}+v_{o}^{\hat{a}(1)} v_{\| o}^{(1)}-v_{o}^{\hat{a}(1)} B_{\| o}^{(1)}+B_{o}^{\hat{a}(1)} v_{\| o}^{(1)}-B_{o}^{\hat{a}(1)} B_{\| o}^{(1)}+\frac{3}{4} n^{i} h_{i o}^{k(1)} h_{k o}^{\hat{a}(1)} .
\end{aligned}
$$


From Eqs. (86), 87) and the constraint from Eq. (91), we obtain at first order

$$
\begin{aligned}
\delta \nu^{(1)}= & -\left(A_{o}^{(1)}-v_{\| o}^{(1)}\right)+2 A^{(1)}-B_{\|}^{(1)}+\int_{0}^{\bar{\chi}} \mathrm{d} \tilde{\chi}\left(A^{(1) \prime}-B_{\|}^{(1) \prime}-\frac{1}{2} h_{\|}^{(1) \prime}\right) \\
= & -\left(A_{o}^{(1)}-v_{\| o}^{(1)}\right)+2 A^{(1)}-B_{\|}^{(1)}-2 I^{(1)}, \\
\delta n^{i(1)}= & +B_{o}^{i(1)}-v_{o}^{i(1)}+\frac{1}{2} n^{j} h_{j o}^{i(1)}-B^{i(1)}-n^{j} h_{j}^{i(1)} \\
& -\int_{0}^{\bar{\chi}} \mathrm{d} \tilde{\chi}\left[\tilde{\partial}^{i}\left(A^{(1)}-B_{\|}^{(1)}-\frac{1}{2} h_{\|}^{(1)}\right)+\frac{1}{\tilde{\chi}}\left(B_{\perp}^{i(1)}+\mathcal{P}^{i j} h_{j k}^{(1)} n^{k}\right)\right] \\
= & n^{i} \delta n_{\|}^{(1)}+\delta n_{\perp}^{i(1)},
\end{aligned}
$$

where

$$
\begin{aligned}
\delta n_{\|}^{(1)} & =A_{o}^{(1)}-v_{\| o}^{(1)}-A^{(1)}-\frac{1}{2} h_{\|}^{(1)}+2 I^{(1)} \\
\delta n_{\perp}^{i(1)} & =B_{\perp o}^{i(1)}-v_{\perp o}^{i(1)}+\frac{1}{2} n^{k} h_{k o}^{j(1)} \mathcal{P}_{j}^{i}-\left(B_{\perp}^{i(1)}+n^{k} h_{k}^{j(1)} \mathcal{P}_{j}^{i}\right)+2 S_{\perp}^{i(1)}
\end{aligned}
$$

Here we have defined

$$
\begin{aligned}
I^{(n)} & =-\frac{1}{2} \int_{0}^{\bar{\chi}} \mathrm{d} \tilde{\chi}\left(A^{(n) \prime}-B_{\|}^{(n) \prime}-\frac{1}{2} h_{\|}^{(n) \prime}\right), \\
S^{i(n)} & =-\frac{1}{2} \int_{0}^{\bar{\chi}} \mathrm{d} \tilde{\chi}\left[\tilde{\partial}^{i}\left(A^{(n)}-B_{\|}^{(n)}-\frac{1}{2} h_{\|}^{(n)}\right)+\frac{1}{\tilde{\chi}}\left(B^{i(n)}+n^{k} h_{k}^{i(n)}\right)\right],
\end{aligned}
$$

where $I^{(n)}$ is the ISW term at order $n, \tilde{\partial}_{i}=\partial / \partial \tilde{x}^{i}$ and

$$
\begin{aligned}
S_{\perp}^{i(n)} & =-\frac{1}{2} \int_{0}^{\bar{\chi}} \mathrm{d} \tilde{\chi}\left[\tilde{\partial}_{\perp}^{i}\left(A^{(n)}-B_{\|}^{(n)}-\frac{1}{2} h_{\|}^{(n)}\right)+\frac{1}{\tilde{\chi}}\left(B_{\perp}^{i(n)}+n^{k} h_{k j}^{(n)} \mathcal{P}^{i j}\right)\right] \\
S_{\|}^{(n)} & =\frac{1}{2}\left(A_{o}^{(n)}-B_{\| o}^{(n)}-\frac{1}{2} h_{\| o}^{(n)}\right)-\frac{1}{2}\left(A^{(n)}-B_{\|}^{(n)}-\frac{1}{2} h_{\|}^{(n)}\right)+I^{(n)}-\frac{1}{2} \int_{0}^{\bar{\chi}} \mathrm{d} \tilde{\chi} \frac{1}{\tilde{\chi}}\left(B_{\|}^{(n)}+h_{\|}^{(n)}\right) .
\end{aligned}
$$

Note the following useful relation

$$
\delta n_{\|}^{(1)}+\delta \nu^{(1)}=A^{(1)}-B_{\|}^{(1)}-\frac{1}{2} h_{\|}^{(1)}
$$

At second order we find

$$
\begin{aligned}
\delta \nu^{(2)}= & -A_{o}^{(2)}+v_{\| o}^{(2)}+\left(A_{o}^{(1)}\right)^{2}-2 A_{o}^{(1)} B_{\| o}^{(1)}+\left(B_{\| o}^{(1)}\right)^{2}+6 A_{o}^{(1)} v_{\| o}^{(1)}-2 B_{\| o}^{(1)} v_{\| o}^{(1)}-v_{k o}^{(1)} v_{o}^{k(1)} \\
& +n^{i} h_{i k o}^{(1)} v_{o}^{k(1)}+4\left(A_{o}^{(1)}-v_{\| o}^{(1)}\right)\left(2 A^{(1)}-B_{\|}^{(1)}-2 I^{(1)}\right)-2\left(B_{\perp o}^{i(1)}-v_{\perp o}^{i(1)}+\frac{1}{2} n^{k} h_{k o}^{j(1)} \mathcal{P}_{j}^{i}\right) B_{\perp i}^{(1)} \\
& +2\left(B_{\perp o}^{i(1)}-v_{\perp o}^{i(1)}+\frac{1}{2} n^{k} h_{k o}^{j(1)} \mathcal{P}_{j}^{i}\right) \int_{0}^{\bar{\chi}} \mathrm{d} \tilde{\chi}\left[\tilde{\partial}_{\perp i}\left(2 A^{(1)}-B_{\|}^{(1)}\right)-\left(B_{\perp i}^{(1) \prime}+n^{j} h_{j k}^{(1) \prime} \mathcal{P}_{i}^{k}\right)+\frac{1}{\tilde{\chi}} B_{\perp i}^{(1)}\right] \\
& +\left(2 A^{(2)}-B_{\|}^{(2)}\right)-3\left(2 A^{(1)}-B_{\|}^{(1)}\right)^{2}-2 B_{\|}^{(1)}\left(A^{(1)}-B_{\|}^{(1)}-\frac{1}{2} h_{\|}^{(1)}\right)+2 B_{\perp i}^{(1)}\left(B_{\perp}^{i(1)}+\mathcal{P}_{j}^{i} h_{k}^{j(1)} n^{k}\right) \\
& +8\left(2 A^{(1)}-B_{\|}^{(1)}\right) I^{(1)}-4 B_{\perp i}^{(1)} S_{\perp}^{i(1)}-2 I^{(2)}+2 \int_{0}^{\bar{\chi}} \mathrm{d} \tilde{\chi}\left\{\left(A^{(1)}-B_{\|}^{(1)}-\frac{1}{2} h_{\|}^{(1)}\right) \frac{\mathrm{d}}{\mathrm{d} \tilde{\chi}}\left(2 A^{(1)}-B_{\|}^{(1)}\right)\right. \\
& +\left(A^{(1) \prime}-B_{\|}^{(1) \prime}-\frac{1}{2} h_{\|}^{(1) \prime}\right)\left[-\left(B_{\|}^{(1)}+h_{\|}^{(1)}\right)+4 I^{(1)}\right]+\left[-\left(B_{\perp}^{i(1)}+n^{j} h_{j k}^{(1)} \mathcal{P}^{i k}\right)+2 S_{\perp}^{i(1)}\right] \\
& \left.\times\left[\tilde{\partial}_{\perp i}\left(2 A^{(1)}-B_{\|}^{(1)}\right)-\left(B_{\perp i}^{(1) \prime}+n^{j} h_{j k}^{(1) \prime} \mathcal{P}_{i}^{k}\right)+\frac{1}{\tilde{\chi}} B_{\perp i}^{(1)}\right]\right\}+\delta \nu_{\mathrm{post}-\mathrm{Born}}^{(2)},
\end{aligned}
$$


where

$$
\begin{aligned}
& \delta \nu_{\text {post }- \text { Born }}^{(2)}=-2\left(2 A_{o}^{(1)}-B_{\| o}^{(1)}\right)\left(3 v_{\| o}^{(1)}+2 h_{\| o}^{(1)}-\frac{1}{2} h_{j o}^{j(1)}\right)-2\left(A_{o}^{(1)}-v_{\| o}^{(1)}\right)\left(2 A^{(1)}-B_{\|}^{(1)}-2 I^{(1)}\right) \\
& -4\left(-B_{\| o}^{(1)}+v_{\| o}^{(1)}-\frac{3}{4} h_{\| o}^{(1)}+\frac{1}{4} h_{j o}^{j(1)}\right) \int_{0}^{\bar{\chi}} \frac{\mathrm{d} \tilde{\chi}}{\tilde{\chi}}\left(2 A^{(1)}-B_{\|}^{(1)}\right)-2\left(B_{\perp o}^{i(1)}-v_{\perp o}^{i(1)}+\frac{1}{2} n^{k} h_{k o}^{j(1)} \mathcal{P}_{j}^{i}\right) \\
& \times \int_{0}^{\bar{\chi}} \mathrm{d} \tilde{\chi}\left[\tilde{\partial}_{\perp i}\left(2 A^{(1)}-B_{\|}^{(1)}\right)+\frac{1}{\tilde{\chi}} B_{\perp i}^{(1)}\right]+2\left(2 A^{(1)}-B_{\|}^{(1)}\right)\left(A^{(1)}+\frac{1}{2} h_{\|}^{(1)}-2 I^{(1)}-2 \kappa^{(1)}\right) \\
& +2\left[\frac{\mathrm{d}}{\mathrm{d} \bar{\chi}}\left(2 A^{(1)}-B_{\|}^{(1)}\right)+\left(A^{(1) \prime}-B_{\|}^{(1) \prime}-\frac{1}{2} h_{\|}^{(1) \prime}\right)\right] \delta x_{\|}^{(1)}+2\left(2 A^{(1) \prime}-B_{\|}^{(1) \prime}\right)\left(\delta x^{0(1)}+\delta x_{\|}^{(1)}\right) \\
& +2\left[\partial_{\perp i}\left(2 A^{(1)}-B_{\|}^{(1)}\right)+\frac{1}{\bar{\chi}} B_{\perp i}^{(1)}\right] \delta x_{\perp}^{i(1)}+2 \int_{0}^{\bar{\chi}} \mathrm{d} \tilde{\chi}\left\{\left(A^{(1) \prime \prime}-B_{\|}^{(1) \prime \prime}-\frac{1}{2} h_{\|}^{(1) \prime \prime}\right)\left(\delta x^{0(1)}+\delta x_{\|}^{(1)}\right)\right. \\
& -\left(2 A^{(1) \prime}-B_{\|}^{(1) \prime}\right)\left(A^{(1)}-B_{\|}^{(1)}-\frac{1}{2} h_{\|}^{(1)}\right)+\left(A^{(1) \prime}-B_{\|}^{(1) \prime}-\frac{1}{2} h_{\|}^{(1) \prime}\right)\left(A^{(1)}+\frac{1}{2} h_{\|}^{(1)}-2 I^{(1)}\right) \\
& -\left(2 A^{(1)}-B_{\|}^{(1)}\right)\left[\frac{\mathrm{d}}{\mathrm{d} \tilde{\chi}}\left(A^{(1)}+\frac{1}{2} h_{\|}^{(1)}\right)+\left(A^{(1) \prime}-B_{\|}^{(1) \prime}-\frac{1}{2} h_{\|}^{(1) \prime}\right)\right]+\left(B_{\perp}^{i(1)}+n^{k} h_{k}^{j(1)} \mathcal{P}_{j}^{i}-2 S_{\perp}^{i(1)}\right) \\
& \times\left[\tilde{\partial}_{\perp i}\left(2 A^{(1)}-B_{\|}^{(1)}\right)+\frac{1}{\tilde{\chi}} B_{\perp i}^{(1)}\right]+\left[\tilde{\partial}_{\perp j}\left(B^{j(1)}+n^{k} h_{k}^{j(1)}\right)\left(2 A^{(1)}-B_{\|}^{(1)}\right)-\frac{2}{\tilde{\chi}}\left(B_{\|}^{(1)}+h_{\|}^{(1)}\right)\left(2 A^{(1)}-B_{\|}^{(1)}\right)\right. \\
& \left.-2\left(2 A^{(1)}-B_{\|}^{(1)}\right) \tilde{\partial}_{\perp j} S_{\perp}^{j(1)}\right]+2\left[\frac{\mathrm{d}}{\mathrm{d} \tilde{\chi}}\left(2 A^{(1)}-B_{\|}^{(1)}\right)-\frac{1}{\tilde{\chi}}\left(2 A^{(1)}-B_{\|}^{(1)}\right)\right] \kappa^{(1)}+\left[\tilde{\partial}_{\perp i}\left(A^{(1) \prime}-B_{\|}^{(1) \prime}-\frac{1}{2} h_{\|}^{(1) \prime}\right)\right. \\
& \left.\left.+\frac{1}{\tilde{\chi}}\left(B_{\perp i}^{(1) \prime}+n^{j} h_{j k}^{(1) \prime} \mathcal{P}_{i}^{k}\right)\right] \delta x_{\perp}^{i(1)}\right\} \cdot
\end{aligned}
$$

Splitting $\delta n^{i(2)}=n^{i} \delta n_{\|}^{(2)}+\delta n_{\perp}^{i(2)}$, we obtain

$$
\begin{aligned}
\delta n_{\|}^{(2)}= & +A_{o}^{(2)}-v_{\| o}^{(2)}+\left(v_{\| o}^{(1)}\right)^{2}-\frac{1}{4}\left(h_{\| o}^{(1)}\right)^{2}-\frac{1}{4} n^{i} h_{i j o}^{(1)} \mathcal{P}_{k}^{j} h_{p o}^{k(1)} n^{p}-4 A_{o}^{(1)} v_{\| o}^{(1)}-2 v_{\| o}^{(1)} h_{\| o}^{(1)}-2 n^{i} h_{i k o}^{(1)} \mathcal{P}_{j}^{k} v_{o}^{j(1)} \\
& -2\left(A_{o}^{(1)}-v_{\| o}^{(1)}\right)\left(2 A^{(1)}+h_{\|}^{(1)}\right)-2\left(B_{\perp o}^{i(1)}-v_{\perp o}^{i(1)}+\frac{1}{2} n^{k} h_{k o}^{j(1)} \mathcal{P}_{j}^{i}\right) h_{i}^{p(1)} n_{p}+8\left(A_{o}^{(1)}-v_{\| o}^{(1)}\right) I^{(1)} \\
& +2\left(B_{\perp o}^{i(1)}-v_{\perp o}^{i(1)}+\frac{1}{2} n^{k} h_{k o}^{j(1)} \mathcal{P}_{j}^{i}\right) \int_{0}^{\bar{\chi}} \mathrm{d} \tilde{\chi}\left[\partial_{\|}\left(B_{\perp i}^{(1)}+n^{j} h_{j k}^{(1)} \mathcal{P}_{i}^{k}\right)-\partial_{\perp i}\left(B_{\|}^{(1)}+h_{\|}^{(1)}\right)\right. \\
& \left.+\frac{1}{\tilde{\chi}}\left(B_{\perp i}^{(1)}+2 n^{j} h_{k j}^{(1)} \mathcal{P}_{i}^{k}\right)\right]-A^{(2)}-\frac{1}{2} h_{\|}^{(2)}-2 h_{\|}^{(1)}\left(A^{(1)}-B_{\|}^{(1)}-\frac{1}{2} h_{\|}^{(1)}\right)+\left(2 A^{(1)}-B_{\|}^{(1)}\right)^{2} \\
& +2\left(2 A^{(1)}-B_{\|}^{(1)}\right)\left(B_{\|}^{(1)}+h_{\|}^{(1)}\right)+2\left(B_{\perp}^{i(1)}+n^{k} h_{k}^{j(1)} \mathcal{P}_{j}^{i}\right) h_{i}^{p(1)} n_{p}-4\left(2 A^{(1)}+h_{\|}^{(1)}\right) I^{(1)}-4 n_{j} h_{i}^{j(1)} S_{\perp}^{i(1)} \\
& +2 I^{(2)}+2 \int_{0}^{\bar{\chi}} \mathrm{d} \tilde{\chi}\left\{\left(2 A^{(1)}-B_{\|}^{(1)}-4 I^{(1)}\right)\left(A^{(1) \prime}-B_{\|}^{(1) \prime}-\frac{1}{2} h_{\|}^{(1) \prime}\right)+\left[-\left(B_{\perp}^{i(1)}+n^{j} h_{j k}^{(1)} \mathcal{P}^{i k}\right)+2 S_{\perp}^{i(1)}\right]\right. \\
& \left.\times\left[\tilde{\partial}_{\|}\left(B_{\perp i}^{(1)}+n^{j} h_{j k}^{(1)} \mathcal{P}_{i}^{k}\right)-\tilde{\partial}_{\perp i}\left(B_{\|}^{(1)}+h_{\|}^{(1)}\right)+\frac{1}{\tilde{\chi}}\left(B_{\perp i}^{(1)}+2 n^{j} h_{j}^{k(1)} \mathcal{P}_{i k}\right)\right]\right\}+\delta n_{\| \text {post-Born }}^{(2)},
\end{aligned}
$$


where

$$
\begin{aligned}
& \delta n_{\| \text {post-Born }}^{(2)}=2\left(A_{o}^{(1)}+\frac{1}{2} h_{\| o}^{(1)}\right)\left(3 v_{\| o}^{(1)}+2 h_{\| o}^{(1)}-\frac{1}{2} h_{j o}^{j(1)}\right)-\left(A_{o}^{(1)}+\frac{1}{2} h_{\| o}^{(1)}\right)^{2}+2\left(A_{o}^{(1)}-v_{\| o}^{(1)}\right)\left(A^{(1)}+\frac{1}{2} h_{\|}^{(1)}\right. \\
& \left.-2 I^{(1)}\right)+4\left(-B_{\| o}^{(1)}+v_{\| o}^{(1)}-\frac{3}{4} h_{\| o}^{(1)}+\frac{1}{4} h_{j o}^{j(1)}\right) \int_{0}^{\bar{\chi}} \frac{\mathrm{d} \tilde{\chi}}{\tilde{\chi}}\left(A^{(1)}+\frac{1}{2} h_{\|}^{(1)}\right)+2\left(B_{\perp o}^{i(1)}-v_{\perp o}^{i(1)}+\frac{1}{2} n^{k} h_{k o}^{j(1)} \mathcal{P}_{j}^{i}\right) \\
& \times \int_{0}^{\bar{\chi}} \mathrm{d} \tilde{\chi}\left[\tilde{\partial}_{\perp i}\left(A^{(1)}+\frac{1}{2} h_{\|}^{(1)}\right)-\frac{1}{\tilde{\chi}} n^{j} h_{j}^{k(1)} \mathcal{P}_{i k}\right]-2\left[\frac{\mathrm{d}}{\mathrm{d} \bar{\chi}}\left(A^{(1)}+\frac{1}{2} h_{\|}^{(1)}\right)+\left(A^{(1) \prime}-B_{\|}^{(1) \prime}-\frac{1}{2} h_{\|}^{(1) \prime}\right)\right] \delta x_{\|}^{(1)} \\
& -2\left(A^{(1) \prime}+\frac{1}{2} h_{\|}^{(1) \prime}\right)\left(\delta x^{0(1)}+\delta x_{\|}^{(1)}\right)-\left(A^{(1)}+\frac{1}{2} h_{\|}^{(1)}\right)\left(A^{(1)}+\frac{1}{2} h_{\|}^{(1)}-4 I^{(1)}\right)+4\left(A^{(1)}+\frac{1}{2} h_{\|}^{(1)}\right) \kappa^{(1)} \\
& -2\left[\partial_{\perp i}\left(A^{(1)}+\frac{1}{2} h_{\|}^{(1)}\right)-\frac{1}{\bar{\chi}} n^{j} h_{j}^{k(1)} \mathcal{P}_{i k}\right] \delta x_{\perp}^{i(1)}+2 \int_{0}^{\bar{\chi}} \mathrm{d} \tilde{\chi}\left\{-\left(A^{(1) \prime \prime}-B_{\|}^{(1) \|}-\frac{1}{2} h_{\|}^{(1) \prime \prime}\right)\left(\delta x^{0(1)}+\delta x_{\|}^{(1)}\right)\right. \\
& +\left(A^{(1) \prime}+\frac{1}{2} h_{\|}^{(1) \prime}\right)\left(A^{(1)}-B_{\|}^{(1)}-\frac{1}{2} h_{\|}^{(1)}\right)+2\left(A^{(1) \prime}-B_{\|}^{(1) \prime}-\frac{1}{2} h_{\|}^{(1) \prime}\right) I^{(1)}-\left(B_{\perp}^{i(1)}+n^{k} h_{k}^{j(1)} \mathcal{P}_{j}^{i}-2 S_{\perp}^{i(1)}\right) \\
& \times\left[\tilde{\partial}_{\perp i}\left(A^{(1)}+\frac{1}{2} h_{\|}^{(1)}\right)-\frac{1}{\tilde{\chi}} n^{j} h_{j}^{k(1)} \mathcal{P}_{i k}\right]-\left(A^{(1)}+\frac{1}{2} h_{\|}^{(1)}\right)\left[\tilde{\partial}_{\perp j}\left(B^{j(1)}+n^{k} h_{k}^{j(1)}\right)-\frac{2}{\tilde{\chi}}\left(B_{\|}^{(1)}+h_{\|}^{(1)}\right)\right. \\
& \left.-2 \tilde{\partial}_{\perp j} S_{\perp}^{j(1)}\right]+2\left[-\frac{\mathrm{d}}{\mathrm{d} \tilde{\chi}}\left(A^{(1)}+\frac{1}{2} h_{\|}^{(1)}\right)+\frac{1}{\tilde{\chi}}\left(A^{(1)}+\frac{1}{2} h_{\|}^{(1)}\right)\right] \kappa^{(1)}-\left[\tilde{\partial}_{\perp i}\left(A^{(1) \prime}-B_{\|}^{(1) \prime}-\frac{1}{2} h_{\|}^{(1) \prime}\right)\right. \\
& \left.\left.+\frac{1}{\tilde{\chi}}\left(B_{\perp i}^{(1) \prime}+n^{j} h_{j k}^{(1) \prime} \mathcal{P}_{i}^{k}\right)\right] \delta x_{\perp}^{i(1)}\right\}
\end{aligned}
$$

and $^{6}$

$$
\begin{aligned}
\delta n_{\perp}^{i(2)}= & +B_{\perp o}^{i(2)}-v_{\perp o}^{i(2)}+\frac{1}{2} n^{j} h_{j k o}^{(2)} \mathcal{P}^{k i}+v_{\| o}^{(1)} v_{\perp o}^{i(1)}-3 v_{\| o}^{(1)} B_{\perp o}^{i(1)}+B_{\| o}^{(1)} B_{\perp o}^{i(1)}-B_{\| o}^{(1)} v_{\perp o}^{i(1)}+2 A_{o}^{(1)} n^{j} h_{j k o}^{(1)} \mathcal{P}^{k i} \\
& -4 v_{\| o}^{(1)} n^{j} h_{j k o}^{(1)} \mathcal{P}^{k i}-2 v_{\perp o}^{j(1)} \mathcal{P}_{j}^{l} h_{l o}^{k(1)} \mathcal{P}_{k}^{i(1)}-\frac{1}{4} h_{\| o}^{(1)} n^{j} h_{j k o}^{(1)} \mathcal{P}^{k i}-\frac{1}{4} n^{j} h_{j o}^{k(1)} \mathcal{P}_{k}^{l} h_{l o}^{p(1)} \mathcal{P}_{p}^{i} \\
& -4\left(A_{o}^{(1)}-v_{\| o}^{(1)}\right)\left(B_{\perp}^{i(1)}+n^{k} h_{k}^{j(1)} \mathcal{P}_{j}^{i}-2 S_{\perp}^{i(1)}\right)+2\left(B_{\perp o}^{j(1)}-v_{\perp o}^{j(1)}+\frac{1}{2} n^{k} h_{k o}^{p(1)} \mathcal{P}_{p}^{j}\right)\left\{-\mathcal{P}_{j}^{l} h_{l}^{k(1)} \mathcal{P}_{k}^{i}\right. \\
& \left.+2 \int_{0}^{\bar{\chi}} \mathrm{d} \tilde{\chi}\left[\tilde{\partial}_{\perp}^{[i} B_{\perp}^{j](1)}+\tilde{\partial}_{\perp}^{[i}\left(\mathcal{P}_{m}^{j]} h_{q}^{m(1)} n^{q}\right)-\frac{1}{\tilde{\chi}}\left(n^{[i} B_{\perp}^{i](1)}+n^{[i} \mathcal{P}_{m}^{j]} h_{q}^{m(1)} n^{q}\right)\right]\right\}-B_{\perp}^{i(2)}-n^{j} h_{j k}^{(2)} \mathcal{P}^{k i} \\
& +4 A^{(1)} B_{\perp}^{i(1)}-2 B_{\|}^{(1)} B_{\perp}^{i(1)}+2 A^{(1)} n^{j} h_{j k}^{(1)} \mathcal{P}^{k i}+h_{\|}^{(1)} n^{j} h_{j k}^{(1)} \mathcal{P}^{k i}+2\left(B_{\perp}^{j(1)}+n^{k} h_{k}^{p(1)} \mathcal{P}_{p}^{j}\right) \mathcal{P}_{j}^{l} h_{l}^{k(1)} \mathcal{P}_{k}^{i} \\
& -8\left(B_{\perp}^{i(1)}+n^{j} h_{j k}^{(1)} \mathcal{P}^{i k}\right) I^{(1)}-4 \mathcal{P}_{j}^{l} h_{l}^{k(1)} \mathcal{P}_{k}^{i} S_{\perp}^{j(1)}+2 S_{\perp}^{i(2)}+2 \int_{0}^{\bar{\chi}} \mathrm{d} \tilde{\chi}\left\{-\left(A^{(1) \prime}-B_{\|}^{(1) \prime}-\frac{1}{2} h_{\|}^{(1) \prime}\right)\right. \\
& \times\left(B_{\perp}^{i(1)}+n^{j} h_{j k}^{(1)} \mathcal{P}^{i k}\right)+\left(2 A^{(1)}-B_{\|}^{(1)}\right) \frac{\mathrm{d}}{\mathrm{d} \tilde{\chi}}\left(B_{\perp}^{i(1)}+n^{j} h_{j k}^{(1)} \mathcal{P}^{i k}\right)+2\left(2 A^{(1)}-B_{\|}^{(1)}-2 I^{(1)}\right) \\
& \times\left[\tilde{\partial}_{\perp}^{i}\left(A^{(1)}-B_{\|}^{(1)}-\frac{1}{2} h_{\|}^{(1)}\right)+\frac{1}{\tilde{\chi}}\left(B_{\perp}^{i(1)}+n^{k} h_{k j}^{(1)} \mathcal{P}^{i j}\right)\right]+2\left[-\left(B_{\perp j}^{(1)}+n^{p} h_{p k}^{(1)} \mathcal{P}_{j}^{k}\right)+2 \delta_{j p} S_{\perp}^{p(1)}\right] \\
& \times\left[\tilde{\partial}_{\perp}^{[i} B_{\perp}^{j](1)}+\tilde{\partial}_{\perp}^{[i}\left(\mathcal{P}_{m}^{j]} h_{q}^{m(1)} n^{q}\right)-\frac{1}{\tilde{\chi}}\left(n^{[i} B_{\perp}^{j](1)}+n^{[i} \mathcal{P}_{m}^{j]} h_{q}^{m(1)} n^{q}\right)\right]+\left(A^{(1)}-B_{\|}^{(1)}-\frac{1}{2} h_{\|}^{(1)}\right) \\
& \left.\times\left[\tilde{\partial}_{\perp}^{i}\left(B_{\|}^{(1)}+h_{\|}^{(1)}\right)-\partial_{\|}\left(B_{\perp}^{i(1)}+n^{p} h_{p q}^{(1)} \mathcal{P}^{i q}\right)-\frac{1}{\tilde{\chi}}\left(B_{\perp}^{i(1)}+2 n^{p} h_{p q}^{(1)} \mathcal{P}^{i q}\right)\right]\right\}+\delta n_{\perp \text { post-Born }}^{(2)},
\end{aligned}
$$

where

${ }^{6}$ From Eq. 66,

$\mathcal{P}_{k}^{i} \mathcal{P}^{j l}\left[\partial^{k} B_{l}^{(1)}-\partial_{l} B^{k(1)}+n^{m} \partial^{k} h_{l m}^{(1)}-n^{m} \partial_{l} h_{m}^{k(1)}\right]=2\left[\partial_{\perp}^{[i} B_{\perp}^{j](1)}+\partial_{\perp}^{[i}\left(\mathcal{P}_{m}^{j]} h_{q}^{m(1)} n^{q}\right)-\tilde{\chi}^{-1}\left(n^{[i} B_{\perp}^{j](1)}+n^{[i} \mathcal{P}_{m}^{j]} h_{q}^{m(1)} n^{q}\right)\right]$. 


$$
\begin{aligned}
& \delta n_{\perp \text { post-Born }}^{(2)}=+2\left(B_{\perp o}^{i(1)}+n^{k} h_{k o}^{j(1)} \mathcal{P}_{j}^{i}\right)\left(3 v_{\| o}^{(1)}+2 h_{\| o}^{(1)}-\frac{1}{2} h_{j o}^{j(1)}\right)-2\left(A_{o}^{(1)}-B_{\| o}^{(1)}-\frac{1}{2} h_{\| o}^{(1)}\right)\left(v_{\perp o}^{i(1)}+\frac{1}{2} n^{k} h_{k o}^{j(1)} \mathcal{P}_{j}^{i}\right) \\
& +2\left(A_{o}^{(1)}-v_{\| o}^{(1)}\right)\left(B_{\perp}^{i(1)}+n^{k} h_{k}^{j(1)} \mathcal{P}_{j}^{i}-2 S_{\perp}^{i(1)}\right)+4\left(-B_{\| o}^{(1)}+v_{\| o}^{(1)}-\frac{3}{4} h_{\| o}^{(1)}+\frac{1}{4} h_{m o}^{m(1)}\right) \\
& \times \int_{0}^{\bar{\chi}} \frac{\mathrm{d} \tilde{\chi}}{\tilde{\chi}}\left(B_{\perp}^{i(1)}+n^{k} h_{k}^{j(1)} \mathcal{P}_{j}^{i}\right)+2\left(B_{\perp o}^{j(1)}-v_{\perp o}^{j(1)}+\frac{1}{2} n^{k} h_{k o}^{p(1)} \mathcal{P}_{p}^{j}\right) \int_{0}^{\bar{\chi}} \mathrm{d} \tilde{\chi}\left[\mathcal{P}_{m}^{i} \partial_{\perp j}\left(B^{m(1)}+n^{k} h_{k}^{m(1)}\right)\right. \\
& \left.-\frac{1}{\tilde{\chi}} \mathcal{P}_{m}^{i} h_{j}^{m(1)}+\frac{1}{\tilde{\chi}} \mathcal{P}_{j}^{i}\left(A^{(1)}-B_{\|}^{(1)}-\frac{1}{2} h_{\|}^{(1)}\right)\right]-2\left(B_{\perp}^{i(1)}+n^{k} h_{k}^{j(1)} \mathcal{P}_{j}^{i}\right)\left(A^{(1)}+\frac{1}{2} h_{\|}^{(1)}-2 I^{(1)}\right) \\
& -2\left[\frac{\mathrm{d}}{\mathrm{d} \bar{\chi}}\left(B_{\perp}^{i(1)}+n^{k} h_{k}^{j(1)} \mathcal{P}_{j}^{i}\right)+\partial_{\perp i}\left(A^{(1)}-B_{\|}^{(1)}-\frac{1}{2} h_{\|}^{(1)}\right)+\frac{1}{\bar{\chi}}\left(B_{\perp}^{i(1)}+n^{k} h_{k}^{j(1)} \mathcal{P}_{j}^{i}\right)\right] \delta x_{\|}^{(1)} \\
& -2\left(B_{\perp i}^{(1) \prime}+n^{j} h_{j k}^{(1) \prime} \mathcal{P}_{i}^{k}\right)\left(\delta x^{0(1)}+\delta x_{\|}^{(1)}\right)+4\left(B_{\perp}^{i(1)}+n^{k} h_{k}^{j(1)} \mathcal{P}_{j}^{i}\right) \kappa^{(1)}-2\left[\mathcal{P}_{m}^{i} \partial_{\perp j}\left(B^{m(1)}+n^{k} h_{k}^{m(1)}\right)\right. \\
& \left.-\frac{1}{\bar{\chi}} \mathcal{P}_{m}^{i} h_{j}^{m(1)}+\frac{1}{\bar{\chi}} \mathcal{P}_{j}^{i}\left(A^{(1)}-B_{\|}^{(1)}-\frac{1}{2} h_{\|}^{(1)}\right)\right] \delta x_{\perp}^{j(1)}+2 \int_{0}^{\bar{\chi}} \mathrm{d} \tilde{\chi}\left\{-\left[\tilde{\partial}_{\perp}^{i}\left(A^{(1) \prime}-B_{\|}^{(1) \prime}-\frac{1}{2} h_{\|}^{(1) \prime}\right)\right.\right. \\
& \left.+\frac{1}{\tilde{\chi}^{2}}\left(B_{\perp}^{i(1)}+n^{k} h_{k}^{j(1)} \mathcal{P}_{j}^{i}\right)+\frac{1}{\tilde{\chi}}\left(B_{\perp}^{i(1) \prime}+n^{j} h_{j}^{k(1) \prime} \mathcal{P}_{k}^{i}\right)\right]\left(\delta x^{0(1)}+\delta x_{\|}^{(1)}\right)+\left(A^{(1)}-B_{\|}^{(1)}-\frac{1}{2} h_{\|}^{(1)}\right) \\
& \times\left(B_{\perp}^{i(1) \prime}+n^{j} h_{j}^{k(1) \prime} \mathcal{P}_{k}^{i}\right)-\left[\tilde{\partial}_{\perp}^{i}\left(A^{(1)}-B_{\|}^{(1)}-\frac{1}{2} h_{\|}^{(1)}\right)+\frac{1}{\tilde{\chi}}\left(B_{\perp}^{i(1)}+n^{k} h_{k}^{j(1)} \mathcal{P}_{j}^{i}\right)\right]\left(A^{(1)}+\frac{1}{2} h_{\|}^{(1)}-2 I^{(1)}\right) \\
& +\left(B_{\perp}^{i(1)}+n^{k} h_{k}^{j(1)} \mathcal{P}_{j}^{i}\right)\left[\frac{\mathrm{d}}{\mathrm{d} \tilde{\chi}}\left(A^{(1)}+\frac{1}{2} h_{\|}^{(1)}\right)+\left(A^{(1) \prime}-B_{\|}^{(1) \prime}-\frac{1}{2} h_{\|}^{(1) \prime}\right)\right] \\
& -\left[\mathcal{P}_{m}^{i} \tilde{\partial}_{\perp j}\left(B^{m(1)}+n^{k} h_{k}^{m(1)}\right)-\frac{1}{\tilde{\chi}} \mathcal{P}_{m}^{i} h_{j}^{m(1)}+\frac{1}{\tilde{\chi}} \mathcal{P}_{j}^{i}\left(A^{(1)}-B_{\|}^{(1)}-\frac{1}{2} h_{\|}^{(1)}\right)\right]\left[\left(B_{\perp}^{j(1)}+n^{k} h_{k}^{m(1)} \mathcal{P}_{m}^{j}\right)-2 S_{\perp}^{j(1)}\right] \\
& +\left(B_{\perp}^{i(1)}+n^{k} h_{k}^{j(1)} \mathcal{P}_{j}^{i}\right)\left[-\tilde{\partial}_{\perp m}\left(B^{m(1)}+n^{l} h_{l}^{m(1)}\right)+\frac{2}{\tilde{\chi}}\left(B_{\|}^{(1)}+h_{\|}^{(1)}\right)+2 \tilde{\partial}_{\perp m} S_{\perp}^{m(1)}\right] \\
& +2\left[-\frac{\mathrm{d}}{\mathrm{d} \tilde{\chi}}\left(B_{\perp}^{i(1)}+n^{k} h_{k}^{j(1)} \mathcal{P}_{j}^{i}\right)+\frac{1}{\tilde{\chi}}\left(B_{\perp}^{i(1)}+n^{k} h_{k}^{j(1)} \mathcal{P}_{j}^{i}\right)\right] \kappa^{(1)} \\
& -\left[\mathcal{P}^{i m} \tilde{\partial}_{\perp j} \tilde{\partial}_{\perp m}\left(A^{(1)}-B_{\|}^{(1)}-\frac{1}{2} h_{\|}^{(1)}\right)+\frac{1}{\tilde{\chi}} \mathcal{P}_{j}^{i}\left(A^{(1) \prime}-B_{\|}^{(1) \prime}-\frac{1}{2} h_{\|}^{(1) \prime}\right)+\frac{1}{\tilde{\chi}^{2}} \mathcal{P}_{j}^{i}\left(A^{(1)}-B_{\|}^{(1)}-\frac{1}{2} h_{\|}^{(1)}\right)\right. \\
& \left.\left.+\frac{1}{\tilde{\chi}} \mathcal{P}_{m}^{i} \tilde{\partial}_{\perp j}\left(B_{\perp}^{m(1)}+n^{k} h_{k}^{l(1)} \mathcal{P}_{l}^{m}\right)+\frac{1}{\tilde{\chi}} \mathcal{P}_{j}^{k} \partial_{\perp}^{i} B_{k}^{(1)}+\frac{1}{\tilde{\chi}} \partial_{\perp}^{i}\left(h_{j l}^{(1)} n^{l}\right)-\frac{1}{\tilde{\chi}^{2}} \mathcal{P}_{l}^{i} h_{j}^{l(1)}\right] \delta x_{\perp}^{j(1)}\right\} .
\end{aligned}
$$

Combining Eqs. 88 and 105 we obtain the useful relation 


$$
\begin{aligned}
\delta \nu^{(2)}+\delta n_{\|}^{(2)}= & +\left(A_{o}^{(1)}\right)^{2}-2 A_{o}^{(1)} B_{\| o}^{(1)}+\left(B_{\| o}^{(1)}\right)^{2}+2 A_{o}^{(1)} v_{\| o}^{(1)}-2 B_{\| o}^{(1)} v_{\| o}^{(1)}-v_{\| o}^{(1)} h_{\| o}^{(1)}-\frac{1}{4}\left(h_{\| o}^{(1)}\right)^{2}-v_{\perp k o}^{(1)} v_{\perp o}^{k(1)} \\
& +n^{i} h_{i k o}^{(1)} \mathcal{P}_{j}^{k} v_{o}^{j(1)}-n^{i} h_{i k o}^{(1)} \mathcal{P}_{j}^{k} B_{o}^{j(1)}-\frac{1}{4} n^{i} h_{i j o}^{(1)} \mathcal{P}_{k}^{j} h_{p o}^{k(1)} n^{p}-B_{\perp o}^{i(1)} B_{\perp i o}^{(1)}+2 v_{\perp o}^{i(1)} B_{\perp i o}^{(1)} \\
& +4\left(A_{o}^{(1)}-v_{\| o}^{(1)}\right)\left(A^{(1)}-B_{\|}^{(1)}-\frac{1}{2} h_{\|}^{(1)}\right)-8\left(B_{\perp o}^{i(1)}-v_{\perp o}^{i(1)}+\frac{1}{2} n^{k} h_{k o}^{j(1)} \mathcal{P}_{j}^{i}\right) \delta_{i l} S_{\perp}^{l(1)} \\
& +A^{(2)}-B_{\|}^{(2)}-\frac{1}{2} h_{\|}^{(2)}-2\left(2 A^{(1)}-B_{\|}^{(1)}\right)^{2}+\left(B_{\|}^{(1)}+h_{\|}^{(1)}\right)\left(2 A^{(1)}+h_{\|}^{(1)}\right) \\
& +\left(B_{\perp}^{i(1)}+n^{k} h_{k}^{j(1)} \mathcal{P}_{j}^{i}\right)\left(B_{\perp i}^{(1)}+n^{p} h_{p m}^{(1)} \mathcal{P}_{i}^{m}\right)+8\left(A^{(1)}-B_{\|}^{(1)}-\frac{1}{2} h_{\|}^{(1)}\right) I^{(1)}-8 S_{\perp}^{i(1)} S_{\perp}^{j(1)} \delta_{i j} \\
& +2 \int_{0}^{\bar{\chi}} \mathrm{d} \tilde{\chi}\left\{\left(A^{(1)}-B_{\|}^{(1)}-\frac{1}{2} h_{\|}^{(1)}\right)\left[2\left(A^{(1) \prime}-B_{\|}^{(1) \prime}-\frac{1}{2} h_{\|}^{(1) \prime}\right)+\frac{\mathrm{d}}{\mathrm{d} \tilde{\chi}}\left(2 A^{(1)}-B_{\|}^{(1)}\right)\right]\right. \\
& \left.-\left(B_{\perp}^{i(1)}+n^{k} h_{k}^{j(1)} \mathcal{P}_{j}^{i}\right)\left[\tilde{\partial}_{\perp i}\left(A^{(1)}-B_{\|}^{(1)}-\frac{1}{2} h_{\|}^{(1)}\right)+\frac{1}{\tilde{\chi}}\left(B_{\perp i}^{(1)}+n^{m} h_{m p}^{(1)} \mathcal{P}_{i}^{p}\right)\right]\right\} \\
& +\left(\delta \nu^{(2)}+\delta n_{\|}^{(2)}\right)_{\text {post }-\operatorname{Born}}
\end{aligned}
$$

where

$$
\begin{aligned}
& \left(\delta \nu^{(2)}+\delta n_{\|}^{(2)}\right)_{\text {post-Born }}=-2\left(A_{o}^{(1)}-B_{\| o}^{(1)}-\frac{1}{2} h_{\| o}^{(1)}\right)\left(3 v_{\| o}^{(1)}+2 h_{\| o}^{(1)}-\frac{1}{2} h_{j o}^{j(1)}\right) \\
& -2\left(A_{o}^{(1)}-v_{\| o}^{(1)}\right)\left(A^{(1)}-B_{\|}^{(1)}-\frac{1}{2} h_{\|}^{(1)}\right)+4\left(B_{\perp o}^{j(1)}-v_{\perp o}^{j(1)}+\frac{1}{2} n^{l} h_{l o}^{p(1)} \mathcal{P}_{p}^{j}\right) S_{\perp}^{j(1)} \\
& -4\left(-B_{\| o}^{(1)}+v_{\| o}^{(1)}-\frac{3}{4} h_{\| o}^{(1)}+\frac{1}{4} h_{m o}^{m(1)}\right) \int_{0}^{\bar{\chi}} \frac{\mathrm{d} \tilde{\chi}}{\tilde{\chi}}\left(A^{(1)}-B_{\|}^{(1)}-\frac{1}{2} h_{\|}^{(1)}\right) \\
& +2 \frac{\mathrm{d}}{\mathrm{d} \bar{\chi}}\left(A^{(1)}-B_{\|}^{(1)}-\frac{1}{2} h_{\|}^{(1)}\right) \delta x_{\|}^{(1)}+2\left(A^{(1) \prime}-B_{\|}^{(1) \prime}-\frac{1}{2} h_{\|}^{(1) \prime}\right)\left(\delta x^{0(1)}+\delta x_{\|}^{(1)}\right) \\
& +2\left(A^{(1)}-B_{\|}^{(1)}-\frac{1}{2} h_{\|}^{(1)}\right)\left(A^{(1)}+\frac{1}{2} h_{\|}^{(1)}-2 I^{(1)}\right)-4\left(A^{(1)}-B_{\|}^{(1)}-\frac{1}{2} h_{\|}^{(1)}\right) \kappa^{(1)} \\
& +2\left[\partial_{\perp i}\left(A^{(1)}-B_{\|}^{(1)}-\frac{1}{2} h_{\|}^{(1)}\right)+\frac{1}{\bar{\chi}}\left(B_{\perp i}^{(1)}+n^{j} h_{j}^{k(1)} \mathcal{P}_{i k}\right)\right] \delta x_{\perp}^{i(1)} \\
& +2 \int_{0}^{\bar{\chi}} \mathrm{d} \tilde{\chi}\left\{-\left(A^{(1)}-B_{\|}^{(1)}-\frac{1}{2} h_{\|}^{(1)}\right)\left[\frac{\mathrm{d}}{\mathrm{d} \tilde{\chi}}\left(A^{(1)}+\frac{1}{2} h_{\|}^{(1)}\right)+2\left(A^{(1) \prime}-B_{\|}^{(1) \prime}-\frac{1}{2} h_{\|}^{(1) \prime}\right)\right]\right. \\
& -\left[\tilde{\partial}_{\perp j}\left(A^{(1)}-B_{\|}^{(1)}-\frac{1}{2} h_{\|}^{(1)}\right)+\frac{1}{\tilde{\chi}}\left(B_{\perp j}^{(1)}+n^{m} h_{m}^{k(1)} \mathcal{P}_{j k}\right)\right]\left[-\left(B_{\perp}^{j(1)}+n^{l} h_{l}^{p(1)} \mathcal{P}_{p}^{j}\right)+2 S_{\perp}^{j(1)}\right] \\
& +\left(A^{(1)}-B_{\|}^{(1)}-\frac{1}{2} h_{\|}^{(1)}\right)\left[\tilde{\partial}_{\perp m}\left(B^{m(1)}+n^{l} h_{l}^{m(1)}\right)-\frac{2}{\tilde{\chi}}\left(B_{\|}^{(1)}+h_{\|}^{(1)}\right)-2 \tilde{\partial}_{\perp m} S_{\perp}^{m(1)}\right] \\
& \left.+2\left[\frac{\mathrm{d}}{\mathrm{d} \tilde{\chi}}\left(A^{(1)}-B_{\|}^{(1)}-\frac{1}{2} h_{\|}^{(1)}\right)-\frac{1}{\tilde{\chi}}\left(A^{(1)}-B_{\|}^{(1)}-\frac{1}{2} h_{\|}^{(1)}\right)\right] \kappa^{(1)}\right\}
\end{aligned}
$$


From Eqs. (16), (17) and (103), we find to first order that

$$
\begin{aligned}
\delta x^{0(1)}= & -\bar{\chi}\left(A_{o}^{(1)}-v_{\| o}^{(1)}\right)+\int_{0}^{\bar{\chi}} \mathrm{d} \tilde{\chi}\left[2 A^{(1)}-B_{\|}^{(1)}+(\bar{\chi}-\tilde{\chi})\left(A^{(1) \prime}-B_{\|}^{(1) \prime}-\frac{1}{2} h_{\|}^{(1) \prime}\right)\right] \\
\delta x_{\|}^{(1)}= & \bar{\chi}\left(A_{o}^{(1)}-v_{\| o}^{(1)}\right)-\int_{0}^{\bar{\chi}} \mathrm{d} \tilde{\chi}\left[\left(A^{(1)}+\frac{1}{2} h_{\|}^{(1)}\right)+(\bar{\chi}-\tilde{\chi})\left(A^{(1) \prime}-B_{\|}^{(1) \prime}-\frac{1}{2} h_{\|}^{(1) \prime}\right)\right], \\
\delta x_{\perp}^{i(1)}= & \bar{\chi}\left(B_{\perp o}^{i(1)}-v_{\perp o}^{i(1)}+\frac{1}{2} n^{k} h_{k o}^{j(1)} \mathcal{P}_{j}^{i}\right)-\int_{0}^{\bar{\chi}} \mathrm{d} \tilde{\chi}\left\{\left(B_{\perp}^{i(1)}+n^{k} h_{k}^{j(1)} \mathcal{P}_{j}^{i}\right)\right. \\
& \left.+(\bar{\chi}-\tilde{\chi})\left[\tilde{\partial}_{\perp}^{i}\left(A^{(1)}-B_{\|}^{(1)}-\frac{1}{2} h_{\|}^{(1)}\right)+\frac{1}{\tilde{\chi}}\left(B_{\perp}^{i(1)}+n^{k} h_{k j}^{(1)} \mathcal{P}^{i j}\right)\right]\right\} \\
\delta x^{0(1)}+\delta x_{\|}^{(1)}= & \int_{0}^{\bar{\chi}} \mathrm{d} \tilde{\chi}\left(A^{(1)}-B_{\|}^{(1)}-\frac{1}{2} h_{\|}^{(1)}\right)=-T^{(1)},
\end{aligned}
$$

where we defined

$$
T^{(n)}=-\int_{0}^{\bar{\chi}} \mathrm{d} \tilde{\chi}\left(A^{(n)}-B_{\|}^{(n)}-\frac{1}{2} h_{\|}^{(n)}\right)
$$

which is an integrated radial displacement corresponding to a time delay term at order $n$, which generalizes the usual (Shapiro) time delay term $T^{(1)}$ [].

At second order,

$$
\begin{aligned}
\delta x^{0(2)}= & \bar{\chi}\left[-A_{o}^{(2)}+v_{\| o}^{(2)}+\left(A_{o}^{(1)}\right)^{2}-2 A_{o}^{(1)} B_{\| o}^{(1)}+\left(B_{\| o}^{(1)}\right)^{2}+6 A_{o}^{(1)} v_{\| o}^{(1)}-2 B_{\| o}^{(1)} v_{\| o}^{(1)}-v_{k o o}^{(1)} v_{o}^{k(1)}\right. \\
& \left.+n^{i} h_{i k o}^{(1)} v_{o}^{k(1)}\right]+4\left(A_{o}^{(1)}-v_{\| o}^{(1)}\right) \int_{0}^{\bar{\chi}} \mathrm{d} \tilde{\chi}\left[\left(2 A^{(1)}-B_{\|}^{(1)}\right)+(\bar{\chi}-\tilde{\chi})\left(A^{(1) \prime}-B_{\|}^{(1) \prime}-\frac{1}{2} h_{\|}^{(1) \prime}\right)\right] \\
& +2\left(B_{\perp o}^{i(1)}-v_{\perp o}^{i(1)}+\frac{1}{2} n^{k} h_{k o}^{j(1)} \mathcal{P}_{j}^{i}\right) \int_{0}^{\bar{\chi}} \mathrm{d} \tilde{\chi}\left\{-B_{\perp i}^{(1)}+(\bar{\chi}-\tilde{\chi})\left[\tilde{\partial}_{\perp i}\left(2 A^{(1)}-B_{\|}^{(1)}\right)-\left(B_{\perp i}^{(1) \prime}+n^{j} h_{j k}^{(1) \prime} \mathcal{P}_{i}^{k}\right)\right.\right. \\
& \left.\left.+\frac{1}{\tilde{\chi}} B_{\perp i}^{(1)}\right]\right\}+\int_{0}^{\bar{\chi}} \mathrm{d} \tilde{\chi}\left[2 A^{(2)}-B_{\|}^{(2)}-12\left(A^{(1)}\right)^{2}+10 A^{(1)} B_{\|}^{(1)}-\left(B_{\|}^{(1)}\right)^{2}+B_{\|}^{(1)} h_{\|}^{(1)}\right. \\
& \left.+2 B_{\perp i}^{(1)}\left(B_{\perp}^{i(1)}+\mathcal{P}_{j}^{i} h_{k}^{j(1)} n^{k}\right)+8\left(2 A^{(1)}-B_{\|}^{(1)}\right) I^{(1)}-4 B_{\perp i}^{(1)} S_{\perp}^{i(1)}\right] \\
& +\int_{0}^{\bar{\chi}} \mathrm{d} \tilde{\chi}(\bar{\chi}-\tilde{\chi})\left\{A^{(2) \prime}-B_{\|}^{(2) \prime}-\frac{1}{2} h_{\|}^{(2) \prime}+2\left(A^{(1)}-B_{\|}^{(1)}-\frac{1}{2} h_{\|}^{(1)}\right) \frac{\mathrm{d}}{\mathrm{d} \tilde{\chi}}\left(2 A^{(1)}-B_{\|}^{(1)}\right)\right. \\
& +2\left(A^{(1) \prime}-B_{\|}^{(1) \prime}-\frac{1}{2} h_{\|}^{(1) \prime}\right)\left[-\left(B_{\|}^{(1)}+h_{\|}^{(1)}\right)+4 I^{(1)}\right]+2\left[-\left(B_{\perp}^{i(1)}+n^{j} h_{j k}^{(1)} \mathcal{P}^{i k}\right)+2 S_{\perp}^{i(1)}\right] \\
& \left.\times\left[\tilde{\partial}_{\perp i}\left(2 A^{(1)}-B_{\|}^{(1)}\right)-\left(B_{\perp i}^{(1) \prime}+n^{j} h_{j k}^{(1) \prime} \mathcal{P}_{i}^{k}\right)+\frac{1}{\tilde{\chi}} B_{\perp i}^{(1)}\right]\right\}+\delta x_{\text {post-Born }}^{0(2)}
\end{aligned}
$$


where

$$
\begin{aligned}
& \delta x_{\text {post-Born }}^{0(2)}=-2 \bar{\chi}\left(2 A_{o}^{(1)}-B_{\| o}^{(1)}\right)\left(3 v_{\| o}^{(1)}+2 h_{\| o}^{(1)}-\frac{1}{2} h_{j o}^{j(1)}\right)+4\left(A_{o}^{(1)}-v_{\| o}^{(1)}\right)\left[\frac{\bar{\chi}}{2}\left(2 A^{(1)}-B_{\|}^{(1)}\right)\right. \\
& \left.-\int_{0}^{\bar{\chi}} \mathrm{d} \tilde{\chi}\left(2 A^{(1)}-B_{\|}^{(1)}-I^{(1)}\right)\right]-4\left(-B_{\| o}^{(1)}+v_{\| o}^{(1)}-\frac{3}{4} h_{\| o}^{(1)}+\frac{1}{4} h_{m o}^{m(1)}\right) \int_{0}^{\bar{\chi}} \mathrm{d} \tilde{\chi} \frac{(\bar{\chi}-\tilde{\chi})}{\tilde{\chi}}\left(2 A^{(1)}-B_{\|}^{(1)}\right) \\
& -2\left(B_{\perp o}^{i(1)}-v_{\perp o}^{i(1)}+\frac{1}{2} n^{k} h_{k o}^{j(1)} \mathcal{P}_{j}^{i}\right) \int_{0}^{\bar{\chi}} \mathrm{d} \tilde{\chi}(\bar{\chi}-\tilde{\chi})\left[\tilde{\partial}_{\perp i}\left(2 A^{(1)}-B_{\|}^{(1)}\right)+\frac{1}{\tilde{\chi}} B_{\perp i}^{(1)}\right] \\
& -2\left(2 A^{(1)}-B_{\|}^{(1)}\right) \int_{0}^{\bar{\chi}} \mathrm{d} \tilde{\chi}\left(A^{(1)}+\frac{1}{2} h_{\|}^{(1)}-2 I^{(1)}\right)+2 \int_{0}^{\bar{\chi}} \mathrm{d} \tilde{\chi}\left\{\left(A^{(1) \prime}-B_{\|}^{(1) \prime}-\frac{1}{2} h_{\|}^{(1) \prime}\right) \delta x_{\|}^{(1)}\right. \\
& -\left(2 A^{(1) \prime}-B_{\|}^{(1) \prime}\right) T^{(1)}+2\left(2 A^{(1)}-B_{\|}^{(1)}\right)\left(A^{(1)}+\frac{1}{2} h_{\|}^{(1)}-2 I^{(1)}-\kappa^{(1)}\right) \\
& \left.+\left[\tilde{\partial}_{\perp i}\left(2 A^{(1)}-B_{\|}^{(1)}\right)+\frac{1}{\tilde{\chi}} B_{\perp i}^{(1)}\right] \delta x_{\perp}^{i(1)}\right\}+2 \int_{0}^{\bar{\chi}} \mathrm{d} \tilde{\chi}(\bar{\chi}-\tilde{\chi})\left\{-\left(A^{(1) \prime \prime}-B_{\|}^{(1) \prime \prime}-\frac{1}{2} h_{\|}^{(1) \prime \prime}\right) T^{(1)}\right. \\
& -\left(2 A^{(1) \prime}-B_{\|}^{(1) \prime}\right)\left(A^{(1)}-B_{\|}^{(1)}-\frac{1}{2} h_{\|}^{(1)}\right)-\left(A^{(1) \prime}-B_{\|}^{(1) \prime}-\frac{1}{2} h_{\|}^{(1) \prime}\right)\left(-A^{(1)}-\frac{1}{2} h_{\|}^{(1)}+2 I^{(1)}\right) \\
& -\left(2 A^{(1)}-B_{\|}^{(1)}\right)\left[\frac{\mathrm{d}}{\mathrm{d} \tilde{\chi}}\left(A^{(1)}+\frac{1}{2} h_{\|}^{(1)}\right)+\left(A^{(1) \prime}-B_{\|}^{(1) \prime}-\frac{1}{2} h_{\|}^{(1) \prime}\right)\right] \\
& +\left[\tilde{\partial}_{\perp i}\left(2 A^{(1)}-B_{\|}^{(1)}\right)+\frac{1}{\tilde{\chi}} B_{\perp i}^{(1)}\right]\left(B_{\perp}^{i(1)}+n^{k} h_{k}^{j(1)} \mathcal{P}_{j}^{i}-2 S_{\perp}^{i(1)}\right) \\
& -\left(2 A^{(1)}-B_{\|}^{(1)}\right)\left[-\tilde{\partial}_{\perp m}\left(B^{m(1)}+n^{l} h_{l}^{m(1)}\right)+\frac{2}{\tilde{\chi}}\left(B_{\|}^{(1)}+h_{\|}^{(1)}\right)+2 \tilde{\partial}_{\perp m} S_{\perp}^{m(1)}\right] \\
& \left.+2\left[\frac{\mathrm{d}}{\mathrm{d} \tilde{\chi}}\left(2 A^{(1)}-B_{\|}^{(1)}\right)-\frac{1}{\tilde{\chi}}\left(2 A^{(1)}-B_{\|}^{(1)}\right)\right] \kappa^{(1)}+\left[\tilde{\partial}_{\perp i}\left(A^{(1) \prime}-B_{\|}^{(1) \prime}-\frac{1}{2} h_{\|}^{(1) \prime}\right)+\frac{1}{\tilde{\chi}}\left(B_{\perp i}^{(1) \prime}+n^{j} h_{j k}^{(1) \prime} \mathcal{P}_{i}^{k}\right)\right] \delta x_{\perp}^{i(1)}\right\}, \\
& \delta x_{\|}^{(2)}=\bar{\chi}\left[A_{o}^{(2)}-v_{\| o}^{(2)}+\left(v_{\| o}^{(1)}\right)^{2}-\frac{1}{4}\left(h_{\| o}^{(1)}\right)^{2}-\frac{1}{4} n^{i} h_{i j o}^{(1)} \mathcal{P}_{k}^{j} h_{p o}^{k(1)} n^{p}-4 A_{o}^{(1)} v_{\| o}^{(1)}-2 v_{\| o}^{(1)} h_{\| o}^{(1)}-2 n^{i} h_{i k o}^{(1)} \mathcal{P}_{j}^{k} v_{o}^{j(1)}\right] \\
& -2\left(A_{o}^{(1)}-v_{\| o}^{(1)}\right) \int_{0}^{\bar{\chi}} \mathrm{d} \tilde{\chi}\left[\left(2 A^{(1)}+h_{\|}^{(1)}\right)+2(\bar{\chi}-\tilde{\chi})\left(A^{(1) \prime}-B_{\|}^{(1) \prime}-\frac{1}{2} h_{\|}^{(1) \prime}\right)\right] \\
& +2\left(B_{\perp o}^{i(1)}-v_{\perp o}^{i(1)}+\frac{1}{2} n^{k} h_{k o}^{j(1)} \mathcal{P}_{j}^{i}\right) \int_{0}^{\bar{\chi}} \mathrm{d} \tilde{\chi}\left\{-h_{i}^{p(1)} n_{p}+(\bar{\chi}-\tilde{\chi})\left[\tilde{\partial}_{\|}\left(B_{\perp i}^{(1)}+n^{j} h_{j k}^{(1)} \mathcal{P}_{i}^{k}\right)-\tilde{\partial}_{\perp i}\left(B_{\|}^{(1)}+h_{\|}^{(1)}\right)\right.\right. \\
& \left.\left.+\frac{1}{\tilde{\chi}}\left(B_{\perp i}^{(1)}+2 n^{j} h_{j}^{k(1)} \mathcal{P}_{k}^{i}\right)\right]\right\}+\int_{0}^{\bar{\chi}} \mathrm{d} \tilde{\chi}\left\{-A^{(2)}-\frac{1}{2} h_{\|}^{(2)}-2 h_{\|}^{(1)}\left(A^{(1)}-B_{\|}^{(1)}-\frac{1}{2} h_{\|}^{(1)}\right)\right. \\
& +\left(2 A^{(1)}-B_{\|}^{(1)}\right)^{2}+2\left(2 A^{(1)}-B_{\|}^{(1)}\right)\left(B_{\|}^{(1)}+h_{\|}^{(1)}\right)+2\left(B_{\perp}^{i(1)}+n^{k} h_{k}^{j(1)} \mathcal{P}_{j}^{i}\right) h_{i}^{p(1)} n_{p}-4\left(2 A^{(1)}+h_{\|}^{(1)}\right) I^{(1)} \\
& \left.-4 n_{j} h_{i}^{j(1)} S_{\perp}^{i(1)}\right\}+\int_{0}^{\bar{\chi}} \mathrm{d} \tilde{\chi}(\bar{\chi}-\tilde{\chi})\left\{-\left(A^{(2) \prime}-B_{\|}^{(2) \prime}-\frac{1}{2} h_{\|}^{(2) \prime}\right)+2\left(2 A^{(1)}-B_{\|}^{(1)}-4 I^{(1)}\right)\right. \\
& \times\left(A^{(1) \prime}-B_{\|}^{(1) \prime}-\frac{1}{2} h_{\|}^{(1) \prime}\right)+2\left[-\left(B_{\perp}^{i(1)}+n^{j} h_{j k}^{(1)} \mathcal{P}^{i k}\right)+2 S_{\perp}^{i(1)}\right]\left[\tilde{\partial}_{\|}\left(B_{\perp i}^{(1)}+n^{j} h_{j k}^{(1)} \mathcal{P}_{i}^{k}\right)\right. \\
& \left.\left.-\tilde{\partial}_{\perp i}\left(B_{\|}^{(1)}+h_{\|}^{(1)}\right)+\frac{1}{\tilde{\chi}}\left(B_{\perp i}^{(1)}+2 n^{j} h_{j}^{k(1)} \mathcal{P}_{k}^{i}\right)\right]\right\}+\delta x_{\| \text {post-Born }}^{(2)},
\end{aligned}
$$


where

$$
\begin{aligned}
& \delta x_{\| \text {post-Born }}^{(2)}=+2 \bar{\chi}\left(A_{o}^{(1)}+\frac{1}{2} h_{\| o}^{(1)}\right)\left(3 v_{\| o}^{(1)}+2 h_{\| o}^{(1)}-\frac{1}{2} h_{j o}^{j(1)}\right)-\bar{\chi}\left(A_{o}^{(1)}+\frac{1}{2} h_{\| o}^{(1)}\right)^{2}+4\left(A_{o}^{(1)}-v_{\| o}^{(1)}\right)\left[-\frac{\bar{\chi}}{2}\left(A^{(1)}+\frac{1}{2} h_{\|}^{(1)}\right)\right. \\
& \left.+\int_{0}^{\bar{\chi}} \mathrm{d} \tilde{\chi}\left(A^{(1)}+\frac{1}{2} h_{\|}^{(1)}-I^{(1)}\right)\right]+4\left(-B_{\| o}^{(1)}+v_{\| o}^{(1)}-\frac{3}{4} h_{\| o}^{(1)}+\frac{1}{4} h_{m o}^{m(1)}\right) \int_{0}^{\bar{\chi}} \mathrm{d} \tilde{\chi} \frac{(\bar{\chi}-\tilde{\chi})}{\tilde{\chi}}\left(A^{(1)}+\frac{1}{2} h_{\|}^{(1)}\right) \\
& +2\left(B_{\perp o}^{i(1)}-v_{\perp o}^{i(1)}+\frac{1}{2} n^{k} h_{k o}^{j(1)} \mathcal{P}_{j}^{i}\right) \int_{0}^{\bar{\chi}} \mathrm{d} \tilde{\chi}(\bar{\chi}-\tilde{\chi})\left[\tilde{\partial}_{\perp i}\left(A^{(1)}+\frac{1}{2} h_{\|}^{(1)}\right)-\frac{1}{\bar{\chi}} n^{j} h_{j}^{k(1)} \mathcal{P}_{i k}\right] \\
& +2\left(A^{(1)}+\frac{1}{2} h_{\|}^{(1)}\right) \int_{0}^{\bar{\chi}} \mathrm{d} \tilde{\chi}\left(A^{(1)}+\frac{1}{2} h_{\|}^{(1)}-2 I^{(1)}\right)+2 \int_{0}^{\bar{\chi}} \mathrm{d} \tilde{\chi}\left\{-\left(A^{(1) \prime}-B_{\|}^{(1) \prime}-\frac{1}{2} h_{\|}^{(1) \prime}\right) \delta x_{\|}^{(1)}\right. \\
& -\left(A^{(1)}+\frac{1}{2} h_{\|}^{(1)}\right)\left[\frac{3}{2}\left(A^{(1)}+\frac{1}{2} h_{\|}^{(1)}\right)-4 I^{(1)}\right]+\left(A^{(1) \prime}+\frac{1}{2} h_{\|}^{(1) \prime}\right) T^{(1)} \\
& \left.+2\left(A^{(1)}+\frac{1}{2} h_{\|}^{(1)}\right) \kappa^{(1)}-\left[\tilde{\partial}_{\perp j}\left(A^{(1)}+\frac{1}{2} h_{\|}^{(1)}\right)-\frac{1}{\tilde{\chi}} n^{j} h_{j}^{k(1)} \mathcal{P}_{j k}\right] \delta x_{\perp}^{j(1)}\right\} \\
& +2 \int_{0}^{\bar{\chi}} \mathrm{d} \tilde{\chi}(\bar{\chi}-\tilde{\chi})\left\{+\left(A^{(1) \prime}+\frac{1}{2} h_{\|}^{(1) \prime}\right)\left(A^{(1)}-B_{\|}^{(1)}-\frac{1}{2} h_{\|}^{(1)}\right)+\left(A^{(1) \prime \prime}-B_{\|}^{(1) \prime \prime}-\frac{1}{2} h_{\|}^{(1) \prime \prime}\right) T^{(1)}\right. \\
& +2\left(A^{(1) \prime}-B_{\|}^{(1) \prime}-\frac{1}{2} h_{\|}^{(1) \prime}\right) I^{(1)}-\left[\tilde{\partial}_{\perp i}\left(A^{(1)}+\frac{1}{2} h_{\|}^{(1)}\right)-\frac{1}{\tilde{\chi}} n^{j} h_{j}^{k(1)} \mathcal{P}_{i k}\right]\left(B_{\perp}^{i(1)}+n^{k} h_{k}^{j(1)} \mathcal{P}_{j}^{i}-2 S_{\perp}^{i(1)}\right) \\
& +\left(A^{(1)}+\frac{1}{2} h_{\|}^{(1)}\right)\left[-\tilde{\partial}_{\perp m}\left(B^{m(1)}+n^{l} h_{l}^{m(1)}\right)+\frac{2}{\tilde{\chi}}\left(B_{\|}^{(1)}+h_{\|}^{(1)}\right)+2 \tilde{\partial}_{\perp m} S_{\perp}^{m(1)}\right] \\
& +2\left[-\frac{\mathrm{d}}{\mathrm{d} \tilde{\chi}}\left(A^{(1)}+\frac{1}{2} h_{\|}^{(1)}\right)+\frac{1}{\tilde{\chi}}\left(A^{(1)}+\frac{1}{2} h_{\|}^{(1)}\right)\right] \kappa^{(1)} \\
& \left.-\left[\tilde{\partial} \perp i\left(A^{(1) \prime}-B_{\|}^{(1) \prime}-\frac{1}{2} h_{\|}^{(1) \prime}\right)+\frac{1}{\tilde{\chi}}\left(B_{\perp i}^{(1) \prime}+n^{j} h_{j k}^{(1) \prime} \mathcal{P}_{i}^{k}\right)\right] \delta x_{\perp}^{i(1)}\right\} \\
& +
\end{aligned}
$$

and 


$$
\begin{aligned}
& \delta x_{\perp}^{i(2)}=\bar{\chi}\left[B_{\perp o}^{i(2)}-v_{\perp o}^{i(2)}+\frac{1}{2} n^{j} h_{j k o}^{(2)} \mathcal{P}^{k i}+v_{\| o}^{(1)} v_{\perp o}^{i(1)}-3 v_{\| o}^{(1)} B_{\perp o}^{i(1)}+B_{\| o}^{(1)} B_{\perp o}^{i(1)}-B_{\| o}^{(1)} v_{\perp o}^{i(1)}+2 A_{o}^{(1)} n^{j} h_{j k o}^{(1)} \mathcal{P}^{k i}\right. \\
& \left.-4 v_{\| o}^{(1)} n^{j} h_{j k o}^{(1)} \mathcal{P}^{k i}-2 v_{\perp o}^{j(1)} \mathcal{P}_{j}^{l} h_{l o}^{k(1)} \mathcal{P}_{k}^{i}-\frac{1}{4} h_{\| o}^{(1)} n^{j} h_{j k o}^{(1)} \mathcal{P}^{k i}-\frac{1}{4} n^{j} h_{j o}^{k(1)} \mathcal{P}_{k}^{l} h_{l o}^{p(1)} \mathcal{P}_{p}^{i}\right] \\
& -4\left(A_{o}^{(1)}-v_{\| o}^{(1)}\right) \int_{0}^{\bar{\chi}} \mathrm{d} \tilde{\chi}\left\{\left(B_{\perp}^{i(1)}+n^{k} h_{k}^{j(1)} \mathcal{P}_{j}^{i}\right)+(\bar{\chi}-\tilde{\chi})\left[\tilde{\partial}_{\perp}^{i}\left(A^{(1)}-B_{\|}^{(1)}-\frac{1}{2} h_{\|}^{(1)}\right)\right.\right. \\
& \left.\left.+\frac{1}{\tilde{\chi}}\left(B_{\perp}^{i(1)}+n^{k} h_{k j}^{(1)} \mathcal{P}^{i j}\right)\right]\right\}+2\left(B_{\perp o}^{j(1)}-v_{\perp o}^{j(1)}+\frac{1}{2} n^{k} h_{k o}^{l(1)} \mathcal{P}_{l}^{j}\right) \int_{0}^{\bar{\chi}} \mathrm{d} \tilde{\chi}\left\{-\mathcal{P}_{j}^{m} h_{m}^{p(1)} \mathcal{P}_{p}^{i}\right. \\
& \left.+2(\bar{\chi}-\tilde{\chi})\left[\tilde{\partial}_{\perp}^{[i} B_{\perp}^{j](1)}+\tilde{\partial}_{\perp}^{[i}\left(\mathcal{P}_{m}^{j]} h_{q}^{m(1)} n^{q}\right)-\frac{1}{\tilde{\chi}}\left(n^{[i} B_{\perp}^{j](1)}+n^{[i} \mathcal{P}_{m}^{j]} h_{q}^{m(1)} n^{q}\right)\right]\right\} \\
& +\int_{0}^{\bar{\chi}} \mathrm{d} \tilde{\chi}\left\{-B_{\perp}^{i(2)}-n^{j} h_{j k}^{(2)} \mathcal{P}^{k i}+4 A^{(1)} B_{\perp}^{i(1)}-2 B_{\|}^{(1)} B_{\perp}^{i(1)}+2 A^{(1)} n^{j} h_{j k}^{(1)} \mathcal{P}^{k i}+h_{\|}^{(1)} n^{j} h_{j k}^{(1)} \mathcal{P}^{k i}\right. \\
& \left.+2\left(B_{\perp}^{j(1)}+n^{k} h_{k}^{p(1)} \mathcal{P}_{p}^{j}\right) \mathcal{P}_{j}^{l} h_{l}^{k(1)} \mathcal{P}_{k}^{i(1)}-8\left(B_{\perp}^{i(1)}+n^{j} h_{j k}^{(1)} \mathcal{P}^{i k}\right) I^{(1)}-4 \mathcal{P}_{j}^{l} h_{l}^{k(1)} \mathcal{P}_{k}^{i} S_{\perp}^{j(1)}\right\} \\
& +\int_{0}^{\bar{\chi}} \mathrm{d} \tilde{\chi}(\bar{\chi}-\tilde{\chi})\left\{-\left[\tilde{\partial}_{\perp}^{i}\left(A^{(2)}-B_{\|}^{(2)}-\frac{1}{2} h_{\|}^{(2)}\right)+\frac{1}{\tilde{\chi}}\left(B_{\perp}^{i(2)}+n^{k} h_{k j}^{(2)} \mathcal{P}^{i j}\right)\right]-2\left(A^{(1) \prime}-B_{\|}^{(1) \prime}-\frac{1}{2} h_{\|}^{(1) \prime}\right)\right. \\
& \times\left(B_{\perp}^{i(1)}+n^{j} h_{j k}^{(1)} \mathcal{P}^{i k}\right)+2\left(2 A^{(1)}-B_{\|}^{(1)}\right) \frac{\mathrm{d}}{\mathrm{d} \tilde{\chi}}\left(B_{\perp}^{i(1)}+n^{j} h_{j k}^{(1)} \mathcal{P}^{i k}\right)+4\left(2 A^{(1)}-B_{\|}^{(1)}-2 I^{(1)}\right) \\
& \times\left[\tilde{\partial}_{\perp}^{i}\left(A^{(1)}-B_{\|}^{(1)}-\frac{1}{2} h_{\|}^{(1)}\right)+\frac{1}{\tilde{\chi}}\left(B_{\perp}^{i(1)}+n^{k} h_{k j}^{(1)} \mathcal{P}^{i j}\right)\right]+4\left[-\left(B_{\perp j}^{(1)}+n^{p} h_{p k}^{(1)} \mathcal{P}_{j}^{k}\right)+2 \delta_{j p} S_{\perp}^{p(1)}\right] \\
& \times\left[\tilde{\partial}_{\perp}^{[i} B_{\perp}^{j](1)}+\tilde{\partial}_{\perp}^{[i}\left(\mathcal{P}_{m}^{j]} h_{q}^{m(1)} n^{q}\right)-\frac{1}{\tilde{\chi}}\left(n^{[i} B_{\perp}^{j](1)}+n^{[i} \mathcal{P}_{m}^{j]} h_{q}^{m(1)} n^{q}\right)\right]+2\left(A^{(1)}-B_{\|}^{(1)}-\frac{1}{2} h_{\|}^{(1)}\right) \\
& \left.\times\left[\tilde{\partial}_{\perp}^{i}\left(B_{\|}^{(1)}+h_{\|}^{(1)}\right)-\partial_{\|}\left(B_{\perp}^{i(1)}+n^{p} h_{p q}^{(1)} \mathcal{P}^{i q}\right)-\frac{1}{\tilde{\chi}}\left(B_{\perp}^{i(1)}+2 n^{p} h_{p q}^{(1)} \mathcal{P}^{i q}\right)\right]\right\}+\delta x_{\perp \text { post }- \text { Born }}^{i(2)},
\end{aligned}
$$


where

$$
\begin{aligned}
& \delta x_{\perp \text { post-Born }}^{i(2)}=2 \bar{\chi}\left(B_{\perp o}^{i(1)}+n^{k} h_{k o}^{j(1)} \mathcal{P}_{j}^{i}\right)\left(3 v_{\| o}^{(1)}+2 h_{\| o}^{(1)}-\frac{1}{2} h_{j o}^{j(1)}\right)-2 \bar{\chi}\left(A_{o}^{(1)}-B_{\| o}^{(1)}-\frac{1}{2} h_{\| o}^{(1)}\right)\left(v_{\perp o}^{i(1)}+\frac{1}{2} n^{k} h_{k o}^{j(1)} \mathcal{P}_{j}^{i}\right) \\
& +4\left(A_{o}^{(1)}-v_{\| o}^{(1)}\right)\left[-\frac{\bar{\chi}}{2}\left(B_{\perp}^{i(1)}+n^{k} h_{k}^{j(1)} \mathcal{P}_{j}^{i}\right)+\int_{0}^{\bar{\chi}} \mathrm{d} \tilde{\chi}\left(B_{\perp}^{i(1)}+n^{k} h_{k}^{j(1)} \mathcal{P}_{j}^{i}-S_{\perp}^{i(1)}\right)\right] \\
& +4\left(-B_{\| o}^{(1)}+v_{\| o}^{(1)}-\frac{3}{4} h_{\| o}^{(1)}+\frac{1}{4} h_{m o}^{m(1)}\right) \int_{0}^{\bar{\chi}} \mathrm{d} \tilde{\chi} \frac{(\bar{\chi}-\tilde{\chi})}{\tilde{\chi}}\left(B_{\perp}^{i(1)}+n^{k} h_{k}^{j(1)} \mathcal{P}_{j}^{i}\right)+2\left(B_{\perp o}^{j(1)}-v_{\perp o}^{j(1)}+\frac{1}{2} n^{l} h_{l o}^{p(1)} \mathcal{P}_{p}^{j}\right) \\
& \times \int_{0}^{\bar{\chi}} \mathrm{d} \tilde{\chi}(\bar{\chi}-\tilde{\chi})\left[\mathcal{P}_{m}^{i} \tilde{\partial}_{\perp j}\left(B^{m(1)}+n^{k} h_{k}^{m(1)}\right)-\frac{1}{\tilde{\chi}} \mathcal{P}_{m}^{i} h_{j}^{m(1)}+\frac{1}{\tilde{\chi}} \mathcal{P}_{j}^{i}\left(A^{(1)}-B_{\|}^{(1)}-\frac{1}{2} h_{\|}^{(1)}\right)\right] \\
& +2\left(B_{\perp}^{i(1)}+n^{k} h_{k}^{j(1)} \mathcal{P}_{j}^{i}\right) \int_{0}^{\bar{\chi}} \mathrm{d} \tilde{\chi}\left(A^{(1)}+\frac{1}{2} h_{\|}^{(1)}-2 I^{(1)}\right)+2 \int_{0}^{\bar{\chi}} \mathrm{d} \tilde{\chi}\left\{-\left[\tilde{\partial}_{\perp}^{i}\left(A^{(1)}-B_{\|}^{(1)}-\frac{1}{2} h_{\|}^{(1)}\right)\right.\right. \\
& \left.+\frac{1}{\tilde{\chi}}\left(B_{\perp}^{i(1)}+n^{k} h_{k}^{j(1)} \mathcal{P}_{j}^{i}\right)\right] \delta x_{\|}^{(1)}+\left(B_{\perp i}^{(1) \prime}+n^{j} h_{j k}^{(1) \prime} \mathcal{P}_{i}^{k}\right) T^{(1)}-2\left(B_{\perp}^{i(1)}+n^{k} h_{k}^{j(1)} \mathcal{P}_{j}^{i}\right)\left(A^{(1)}+\frac{1}{2} h_{\|}^{(1)}-2 I^{(1)}\right. \\
& \left.\left.-\kappa^{(1)}\right)-\left[\mathcal{P}_{m}^{i} \tilde{\partial}_{\perp j}\left(B^{m(1)}+n^{k} h_{k}^{m(1)}\right)-\frac{1}{\tilde{\chi}} \mathcal{P}_{m}^{i} h_{j}^{m(1)}+\frac{1}{\tilde{\chi}} \mathcal{P}_{j}^{i}\left(A^{(1)}-B_{\|}^{(1)}-\frac{1}{2} h_{\|}^{(1)}\right)\right] \delta x_{\perp}^{j(1)}\right\} \\
& +2 \int_{0}^{\bar{\chi}} \mathrm{d} \tilde{\chi}(\bar{\chi}-\tilde{\chi})\left\{-\left[\tilde{\partial}_{\perp}^{i}\left(A^{(1) \prime}-B_{\|}^{(1) \prime}-\frac{1}{2} h_{\|}^{(1) \prime}\right)-\frac{1}{\tilde{\chi}^{2}}\left(B_{\perp}^{i(1)}+n^{k} h_{k}^{j(1)} \mathcal{P}_{j}^{i}\right)+\frac{1}{\tilde{\chi}}\left(B_{\perp}^{i(1) \prime}+n^{j} h_{j}^{k(1) \prime} \mathcal{P}_{k}^{i}\right)\right] T^{(1)}\right. \\
& +\left(B_{\perp}^{i(1) \prime}+n^{j} h_{j}^{k(1) \prime} \mathcal{P}_{k}^{i}\right)\left(A^{(1)}-B_{\|}^{(1)}-\frac{1}{2} h_{\|}^{(1)}\right)-\left[\tilde{\partial}_{\perp}^{i}\left(A^{(1)}-B_{\|}^{(1)}-\frac{1}{2} h_{\|}^{(1)}\right)+\frac{1}{\tilde{\chi}}\left(B_{\perp}^{i(1)}+n^{k} h_{k}^{j(1)} \mathcal{P}_{j}^{i}\right)\right] \\
& \times\left(A^{(1)}+\frac{1}{2} h_{\|}^{(1)}-2 I^{(1)}\right)+\left(B_{\perp}^{i(1)}+n^{k} h_{k}^{j(1)} \mathcal{P}_{j}^{i}\right)\left[\frac{\mathrm{d}}{\mathrm{d} \tilde{\chi}}\left(A^{(1)}+\frac{1}{2} h_{\|}^{(1)}\right)+\left(A^{(1) \prime}-B_{\|}^{(1) \prime}-\frac{1}{2} h_{\|}^{(1) \prime}\right)\right] \\
& -\left[\mathcal{P}_{m}^{i} \tilde{\partial}_{\perp j}\left(B^{m(1)}+n^{k} h_{k}^{m(1)}\right)-\frac{1}{\tilde{\chi}} \mathcal{P}_{m}^{i} h_{j}^{m(1)}+\frac{1}{\tilde{\chi}} \mathcal{P}_{j}^{i}\left(A^{(1)}-B_{\|}^{(1)}-\frac{1}{2} h_{\|}^{(1)}\right)\right]\left[\left(B_{\perp}^{j(1)}+n^{l} h_{l}^{p(1)} \mathcal{P}_{p}^{j}\right)-2 S_{\perp}^{j(1)}\right] \\
& +\left(B_{\perp}^{i(1)}+n^{k} h_{k}^{j(1)} \mathcal{P}_{j}^{i}\right)\left[-\tilde{\partial}_{\perp m}\left(B^{m(1)}+n^{l} h_{l}^{m(1)}\right)+\frac{2}{\tilde{\chi}}\left(B_{\|}^{(1)}+h_{\|}^{(1)}\right)+2 \tilde{\partial}_{\perp m} S_{\perp}^{m(1)}\right] \\
& +2\left[-\frac{\mathrm{d}}{\mathrm{d} \tilde{\chi}}\left(B_{\perp}^{i(1)}+n^{k} h_{k}^{j(1)} \mathcal{P}_{j}^{i}\right)+\frac{1}{\tilde{\chi}}\left(B_{\perp}^{i(1)}+n^{k} h_{k}^{j(1)} \mathcal{P}_{j}^{i}\right)\right] \kappa^{(1)}-\left[\mathcal{P}^{i m} \tilde{\partial}_{\perp j} \tilde{\partial}_{\perp m}\left(A^{(1)}-B_{\|}^{(1)}-\frac{1}{2} h_{\|}^{(1)}\right)\right. \\
& +\frac{1}{\tilde{\chi}} \mathcal{P}_{j}^{i}\left(A^{(1) \prime}-B_{\|}^{(1) \prime}-\frac{1}{2} h_{\|}^{(1) \prime}\right)+\frac{1}{\tilde{\chi}^{2}} \mathcal{P}_{j}^{i}\left(A^{(1)}-B_{\|}^{(1)}-\frac{1}{2} h_{\|}^{(1)}\right)+\frac{1}{\tilde{\chi}} \mathcal{P}_{m}^{i} \tilde{\partial}_{\perp j}\left(B_{\perp}^{m(1)}+n^{k} h_{k}^{l(1)} \mathcal{P}_{l}^{m}\right) \\
& \left.\left.+\frac{1}{\tilde{\chi}} \mathcal{P}_{j}^{k} \partial_{\perp}^{i} B_{k}^{(1)}+\frac{1}{\tilde{\chi}} \partial_{\perp}^{i}\left(h_{j l}^{(1)} n^{l}\right)-\frac{1}{\tilde{\chi}^{2}} \mathcal{P}_{l}^{i} h_{j}^{l(1)}\right] \delta x_{\perp}^{j(1)}\right\}
\end{aligned}
$$


Combining Eqs. (113) and (114) [or integrating Eq. 107]], we have

$$
\begin{aligned}
\delta x^{0(2)}+\delta x_{\|}^{(2)}= & \bar{\chi}\left[\left(A_{o}^{(1)}\right)^{2}-2 A_{o}^{(1)} B_{\| o}^{(1)}+\left(B_{\| o}^{(1)}\right)^{2}+2 A_{o}^{(1)} v_{\| o}^{(1)}-2 B_{\| o}^{(1)} v_{\| o}^{(1)}-v_{\| o}^{(1)} h_{\| o}^{(1)}-\frac{1}{4}\left(h_{\| o}^{(1)}\right)^{2}-v_{\perp k o}^{(1)} v_{\perp o}^{k(1)}\right. \\
& \left.+n^{i} h_{i k o}^{(1)} \mathcal{P}_{j}^{k} v_{o}^{j(1)}-n^{i} h_{i k o}^{(1)} \mathcal{P}_{j}^{k} B_{o}^{j(1)}-\frac{1}{4} n^{i} h_{i j o}^{(1)} \mathcal{P}_{k}^{j} h_{p o}^{k(1)} n^{p}-B_{\perp o}^{i(1)} B_{\perp i o}^{(1)}+2 v_{\perp o}^{i(1)} B_{\perp i o}^{(1)}\right] \\
& -4\left(A_{o}^{(1)}-v_{\| o}^{(1)}\right) T^{(1)}+4\left(B_{\perp i o}^{(1)}-v_{\perp i o}^{(1)}+\frac{1}{2} n^{k} h_{k o}^{j(1)} \mathcal{P}_{i j}\right) \\
& \times \int_{0}^{\bar{\chi}} \mathrm{d} \tilde{\chi}(\bar{\chi}-\tilde{\chi})\left[\tilde{\partial}_{\perp}^{i}\left(A^{(1)}-B_{\|}^{(1)}-\frac{1}{2} h_{\|}^{(1)}\right)+\frac{1}{\tilde{\chi}}\left(B_{\perp}^{i(1)}+n^{k} h_{k j}^{(1)} \mathcal{P}^{i j}\right)\right] \\
& -T^{(2)}+2 \int_{0}^{\bar{\chi}} \mathrm{d} \tilde{\chi}\left[-\left(2 A^{(1)}-B_{\|}^{(1)}\right)^{2}+\frac{1}{2}\left(B_{\|}^{(1)}+h_{\|}^{(1)}\right)\left(2 A^{(1)}+h_{\|}^{(1)}\right)\right. \\
& \left.+\frac{1}{2}\left(B_{\perp}^{i(1)}+n^{k} h_{k}^{j(1)} \mathcal{P}_{j}^{i}\right)\left(B_{\perp i}^{(1)}+n^{p} h_{p m}^{(1)} \mathcal{P}_{i}^{m}\right)+4\left(A^{(1)}-B_{\|}^{(1)}-\frac{1}{2} h_{\|}^{(1)}\right) I^{(1)}-4 S_{\perp}^{i(1)} S_{\perp}^{j(1)} \delta_{i j}\right] \\
& +2 \int_{0}^{\bar{\chi}} \mathrm{d} \tilde{\chi}(\bar{\chi}-\tilde{\chi})\left\{\left(A^{(1)}-B_{\|}^{(1)}-\frac{1}{2} h_{\|}^{(1)}\right)\left[2\left(A^{(1) \prime}-B_{\|}^{(1) \prime}-\frac{1}{2} h_{\|}^{(1) \prime}\right)+\frac{\mathrm{d}}{\mathrm{d} \tilde{\chi}}\left(2 A^{(1)}-B_{\|}^{(1)}\right)\right]\right. \\
& \left.-\left(B_{\perp}^{i(1)}+n^{k} h_{k}^{j(1)} \mathcal{P}_{j}^{i}\right)\left[\tilde{\partial}_{\perp i}\left(A^{(1)}-B_{\|}^{(1)}-\frac{1}{2} h_{\|}^{(1)}\right)+\frac{1}{\tilde{\chi}}\left(B_{\perp i}^{(1)}+n^{m} h_{m p}^{(1)} \mathcal{P}_{i}^{p}\right)\right]\right\} \\
& +\left(\delta x^{0(2)}+\delta x_{\|}^{(2)}\right)_{\text {post-Born }},
\end{aligned}
$$

where

$$
\begin{aligned}
& \left(\delta x^{0(2)}+\delta x_{\|}^{(2)}\right)_{\text {post-Born }}=-2 \bar{\chi}\left(A_{o}^{(1)}-B_{\| o}^{(1)}-\frac{1}{2} h_{\| o}^{(1)}\right)\left(3 v_{\| o}^{(1)}+2 h_{\| o}^{(1)}-\frac{1}{2} h_{j o}^{j(1)}\right)+2\left(A_{o}^{(1)}-v_{\| o}^{(1)}\right) \\
& \times\left[\bar{\chi}\left(A^{(1)}-B_{\|}^{(1)}-\frac{1}{2} h_{\|}^{(1)}\right)+2 T^{(1)}\right]-4\left(-B_{\| o}^{(1)}+v_{\| o}^{(1)}-\frac{3}{4} h_{\| o}^{(1)}+\frac{1}{4} h_{m o}^{m(1)}\right) \int_{0}^{\bar{\chi}} \mathrm{d} \tilde{\chi} \frac{(\bar{\chi}-\tilde{\chi})}{\tilde{\chi}}\left(A^{(1)}-B_{\|}^{(1)}-\frac{1}{2} h_{\|}^{(1)}\right) \\
& +4\left(B_{\perp o}^{i(1)}-v_{\perp o}^{i(1)}+\frac{1}{2} n^{k} h_{k o}^{j(1)} \mathcal{P}_{j}^{i}\right) \int_{0}^{\bar{\chi}} \mathrm{d} \tilde{\chi} S_{\perp i}^{(1)}-2\left(A^{(1)}-B_{\|}^{(1)}-\frac{1}{2} h_{\|}^{(1)}\right) \int_{0}^{\bar{\chi}} \mathrm{d} \tilde{\chi}\left[\left(A^{(1)}+\frac{1}{2} h_{\|}^{(1)}\right)-2 I^{(1)}\right] \\
& +2 \int_{0}^{\bar{\chi}} \mathrm{d} \tilde{\chi}\left\{-\left(A^{(1) \prime}-B_{\|}^{(1) \prime}-\frac{1}{2} h_{\|}^{(1) \prime}\right) T^{(1)}+2\left(A^{(1)}-B_{\|}^{(1)}-\frac{1}{2} h_{\|}^{(1)}\right)\left(A^{(1)}+\frac{1}{2} h_{\|}^{(1)}-2 I^{(1)}-\kappa^{(1)}\right)\right. \\
& \left.+\left[\tilde{\partial}_{\perp i}\left(A^{(1)}-B_{\|}^{(1)}-\frac{1}{2} h_{\|}^{(1)}\right)+\frac{1}{\tilde{\chi}}\left(B_{\perp i}^{(1)}+n^{j} h_{j}^{k(1)} \mathcal{P}_{i k}\right)\right] \delta x_{\perp}^{i(1)}\right\} \\
& +2 \int_{0}^{\bar{\chi}} \mathrm{d} \tilde{\chi}(\bar{\chi}-\tilde{\chi})\left\{-\left(A^{(1)}-B_{\|}^{(1)}-\frac{1}{2} h_{\|}^{(1)}\right)\left[\frac{\mathrm{d}}{\mathrm{d} \tilde{\chi}}\left(A^{(1)}+\frac{1}{2} h_{\|}^{(1)}\right)+2\left(A^{(1) \prime}-B_{\|}^{(1) \prime}-\frac{1}{2} h_{\|}^{(1) \prime}\right)\right]\right. \\
& -\left[\tilde{\partial}{ }_{\perp j}\left(A^{(1)}-B_{\|}^{(1)}-\frac{1}{2} h_{\|}^{(1)}\right)+\frac{1}{\tilde{\chi}}\left(B_{\perp j}^{(1)}+n^{m} h_{m}^{k(1)} \mathcal{P}_{j k}\right)\right]\left[-\left(B_{\perp}^{i(1)}+n^{k} h_{k}^{j(1)} \mathcal{P}_{j}^{i}\right)+2 S_{\perp}^{i(1)}\right] \\
& -\left(A^{(1)}-B_{\|}^{(1)}-\frac{1}{2} h_{\|}^{(1)}\right)\left[-\tilde{\partial}_{\perp m}\left(B^{m(1)}+n^{l} h_{l}^{m(1)}\right)+\frac{2}{\tilde{\chi}}\left(B_{\|}^{(1)}+h_{\|}^{(1)}\right)+2 \tilde{\partial}_{\perp m} S_{\perp}^{m(1)}\right] \\
& \left.+2\left[\frac{\mathrm{d}}{\mathrm{d} \tilde{\chi}}\left(A^{(1)}-B_{\|}^{(1)}-\frac{1}{2} h_{\|}^{(1)}\right)-\frac{1}{\tilde{\chi}}\left(A^{(1)}-B_{\|}^{(1)}-\frac{1}{2} h_{\|}^{(1)}\right)\right] \kappa^{(1)}\right\} .
\end{aligned}
$$

To obtain all the second order terms we require $\Delta \ln a^{(1)}\left(\right.$ or $\left.\Delta x^{0(1)}\right), \delta \chi^{(1)}, \Delta x^{0(1)}, \Delta x_{\|}^{(1)}, \Delta x_{\perp}^{i(1)}$ and $\Delta_{g}^{(1)}$. From 
Eqs. (32) and (34) we have

$$
\begin{aligned}
\Delta \ln a^{(1)}= & -E_{\hat{0} 0}^{(1)}+E_{\hat{0} \|}^{(1)}-\delta \nu^{(1)}=\left(A_{o}^{(1)}-v_{\| o}^{(1)}\right)-A^{(1)}+v_{\|}^{(1)}+2 I^{(1)} \\
= & +\left(A_{o}^{(1)}-v_{\| o}^{(1)}\right)-A^{(1)}+v_{\|}^{(1)}-\int_{0}^{\bar{\chi}} \mathrm{d} \tilde{\chi}\left(A^{(1) \prime}-B_{\|}^{(1) \prime}-\frac{1}{2} h_{\|}^{(1) \prime}\right) \\
\delta \chi^{(1)}= & \delta x^{0(1)}-\Delta x^{0(1)}=\delta x^{0(1)}-\frac{1}{\mathcal{H}} \Delta \ln a^{(1)}=-\left(\bar{\chi}+\frac{1}{\mathcal{H}}\right)\left(A_{o}^{(1)}-v_{\| o}^{(1)}\right)+\frac{1}{\mathcal{H}}\left(A^{(1)}-v_{\|}^{(1)}\right) \\
& +\int_{0}^{\bar{\chi}} \mathrm{d} \tilde{\chi}\left[2 A^{(1)}-B_{\|}^{(1)}+(\bar{\chi}-\tilde{\chi})\left(A^{(1) \prime}-B_{\|}^{(1) \prime}-\frac{1}{2} h_{\|}^{(1) \prime}\right)\right]-\frac{2}{\mathcal{H}} I^{(1)} .
\end{aligned}
$$

Then, from Eq. (36)

$$
\begin{aligned}
\Delta x^{0(1)} & =\frac{1}{\mathcal{H}}\left[\left(A_{o}^{(1)}-v_{\| o}^{(1)}\right)-A^{(1)}+v_{\|}^{(1)}+2 I^{(1)}\right] \\
& =\frac{1}{\mathcal{H}}\left[\left(A_{o}^{(1)}-v_{\| o}^{(1)}\right)-A^{(1)}+v_{\|}^{(1)}-\int_{0}^{\bar{\chi}} \mathrm{d} \tilde{\chi}\left(A^{(1) \prime}-B_{\|}^{(1) \prime}-\frac{1}{2} h_{\|}^{(1) \prime}\right)\right],
\end{aligned}
$$

and from Eqs. 69 and 111

$$
\begin{aligned}
& \Delta x_{\|}^{(1)}=-T^{(1)}-\Delta x^{0(1)}=-T^{(1)}-\frac{1}{\mathcal{H}} \Delta \ln a^{(1)}=-T^{(1)}-\frac{1}{\mathcal{H}}\left[\left(A_{o}^{(1)}-v_{\| o}^{(1)}\right)-A^{(1)}+v_{\|}^{(1)}+2 I^{(1)}\right] \\
& =\int_{0}^{\bar{\chi}} \mathrm{d} \tilde{\chi}\left(A^{(1)}-B_{\|}^{(1)}-\frac{1}{2} h_{\|}^{(1)}\right)-\frac{1}{\mathcal{H}}\left[\left(A_{o}^{(1)}-v_{\| o}^{(1)}\right)-A^{(1)}+v_{\|}^{(1)}-\int_{0}^{\bar{\chi}} \mathrm{d} \tilde{\chi}\left(A^{(1) \prime}-B_{\|}^{(1) \prime}-\frac{1}{2} h_{\|}^{(1) \prime}\right)\right] .
\end{aligned}
$$

Using Eq. 70, we have

$$
\begin{aligned}
\Delta x_{\perp}^{i(1)}= & \bar{\chi}\left(B_{\perp o}^{i(1)}-v_{\perp o}^{i(1)}+\frac{1}{2} n^{k} h_{k o}^{j(1)} \mathcal{P}_{j}^{i}\right)-\int_{0}^{\bar{\chi}} \mathrm{d} \tilde{\chi}\left\{\left(B_{\perp}^{i(1)}+n^{k} h_{k}^{j(1)} \mathcal{P}_{j}^{i}\right)\right. \\
& \left.+(\bar{\chi}-\tilde{\chi})\left[\tilde{\partial}_{\perp}^{i}\left(A^{(1)}-B_{\|}^{(1)}-\frac{1}{2} h_{\|}^{(1)}\right)+\frac{1}{\tilde{\chi}}\left(B_{\perp}^{i(1)}+n^{k} h_{k j}^{(1)} \mathcal{P}^{i j}\right)\right]\right\}
\end{aligned}
$$

In Eq. (119) there is an ISW contribution and in Eq. (120) we have both time-delay and ISW contributions.

Now we can obtain $\Delta_{g}^{(1)}$. Using Eq. $\sqrt{79}$ for $\Delta x_{\|}^{(1)}$, we find

$$
\begin{aligned}
\partial_{\|} \Delta x_{\|}^{(1)} & =\partial_{\bar{\chi}} \Delta x_{\|}^{(1)}=-\partial_{\bar{\chi}}\left(T^{(1)}+\Delta x^{0(1)}\right)=\left(A^{(1)}-B_{\|}^{(1)}-\frac{1}{2} h_{\|}^{(1)}\right)-\frac{\mathcal{H}^{\prime}}{\mathcal{H}^{2}} \Delta \ln a^{(1)}-\frac{1}{\mathcal{H}}\left(\frac{\mathrm{d} \Delta \ln a}{\mathrm{~d} \bar{\chi}}\right)^{(1)} \\
& =\left(A^{(1)}-B_{\|}^{(1)}-\frac{1}{2} h_{\|}^{(1)}\right)+\frac{1}{\mathcal{H}}\left[\frac{\mathrm{d}}{\mathrm{d} \bar{\chi}}\left(A^{(1)}-v_{\|}^{(1)}\right)+\left(A^{(1) \prime}-B_{\|}^{(1) \prime}-\frac{1}{2} h_{\|}^{(1) \prime}\right)\right]-\frac{\mathcal{H}^{\prime}}{\mathcal{H}^{2}} \Delta \ln a^{(1)} .
\end{aligned}
$$

From Eqs. 117), 120) and (122), we find that Eq. (76) becomes

$$
\begin{aligned}
\Delta_{g}^{(1)}= & \delta_{g}^{(1)}+\left(b_{e}-\frac{\mathcal{H}^{\prime}}{\mathcal{H}^{2}}-\frac{2}{\bar{\chi} \mathcal{H}}\right) \Delta \ln a^{(1)}+\frac{1}{\mathcal{H}}\left[\frac{\mathrm{d}}{\mathrm{d} \bar{\chi}}\left(A^{(1)}-v_{\|}^{(1)}\right)+\left(A^{(1) \prime}-B_{\|}^{(1) \prime}-\frac{1}{2} h_{\|}^{(1) \prime}\right)\right] \\
& -\frac{2}{\bar{\chi}} T^{(1)}-2 \kappa^{(1)}+A^{(1)}+v_{\|}^{(1)}-B_{\|}^{(1)}-\frac{1}{2} h_{\|}^{(1)}+\frac{1}{2} h_{i}^{i(1)},
\end{aligned}
$$

in agreement with [1 1 .

Making explicit at first order the coordinate convergence lensing term defined in Eq. 80] (see also [7]), we find ${ }^{7}$

$$
\kappa^{(1)}=-\frac{1}{2} \partial_{\perp i} \Delta x_{\perp}^{i(1)}=\kappa_{1}^{(1)}+\kappa_{2}^{(1)}+\kappa_{3}^{(1)}
$$

\footnotetext{
7 To compute correctly the lensing term, we need further properties of the parallel and orthogonal derivatives. If $\tilde{x}^{j}$ is not necessarily the same as $\bar{x}^{i}$ (i.e. $\tilde{\chi}$ can be different from $\bar{\chi}$ ), then $\partial \bar{x}^{i}(\bar{\chi}) / \partial \tilde{x}^{j}=\partial \bar{\chi} / \partial \tilde{x}^{j} n^{i}+\bar{\chi} \partial n^{i} / \partial \tilde{x}^{j}=\left(\partial \bar{\chi} / \partial \tilde{x}^{j}\right) n^{i}+(\bar{\chi} / \tilde{\chi}) \mathcal{P}_{j}^{i}$. (We used $\partial n^{i} / \partial \tilde{x}^{j}=\mathcal{P}_{j}^{i} / \tilde{\chi}$.) If $\bar{\chi}=\tilde{\chi}$, then $\partial \bar{\chi} / \partial \tilde{x}^{j}=n_{j}$, and if $\bar{\chi} \neq \tilde{\chi}$, we have $\partial \bar{\chi} / \partial \tilde{x}^{j}=0$. Thus $\partial \bar{x}^{i}(\bar{\chi}) / \partial \tilde{x}^{j}=\delta_{j}^{i}$ for $\bar{\chi}=\tilde{\chi}$, and $=(\bar{\chi} / \tilde{\chi}) \mathcal{P}_{j}^{i}$ for $\bar{\chi} \neq \tilde{\chi}$. Note that for $\bar{\chi} \neq \tilde{\chi}$, the orthogonal part survives. Then

$$
\tilde{\partial}_{\perp j} F\left(\bar{x}^{i}\right)=\mathcal{P}_{j}^{k}\left(\partial \bar{x}^{i}(\bar{\chi}) / \partial \tilde{x}^{k}\right) \partial F / \partial \bar{x}^{i}=(\bar{\chi} / \tilde{\chi}) \mathcal{P}_{j}^{i} \partial F / \partial \bar{x}^{i}=(\bar{\chi} / \tilde{\chi}) \partial_{\perp j} F .
$$
}


where, using $\partial_{\perp i} \mathcal{P}_{j}^{i}=-2 n_{j} / \bar{\chi}$,

$$
\begin{array}{r}
\kappa_{1}^{(1)}=\frac{1}{2} \partial_{\perp i} \int_{0}^{\bar{\chi}} \mathrm{d} \tilde{\chi}(\bar{\chi}-\tilde{\chi}) \tilde{\partial}_{\perp}^{i}\left(A^{(1)}-B_{\|}^{(1)}-\frac{1}{2} h_{\|}^{(1)}\right)=\frac{1}{2} \int_{0}^{\bar{\chi}} \mathrm{d} \tilde{\chi}(\bar{\chi}-\tilde{\chi}) \frac{\tilde{\chi}}{\bar{\chi}} \tilde{\nabla}_{\perp}^{2}\left(A^{(1)}-B_{\|}^{(1)}-\frac{1}{2} h_{\|}^{(1)}\right), \\
\kappa_{2}^{(1)}=\frac{1}{2} \partial_{\perp i} \int_{0}^{\bar{\chi}} \mathrm{d} \tilde{\chi} \frac{\bar{\chi}}{\tilde{\chi}}\left(B_{\perp}^{i(1)}+n^{k} h_{k j}^{(1)} \mathcal{P}^{i j}\right)=\frac{1}{2} \int_{0}^{\bar{\chi}} \mathrm{d} \tilde{\chi}\left[\tilde{\partial}_{\perp}^{i} B_{i}^{(1)}-\frac{2}{\tilde{\chi}} B_{\|}^{(1)}+\mathcal{P}^{i j} n^{k} \tilde{\partial}_{i} h_{j k}^{(1)}+\frac{1}{\tilde{\chi}}\left(h_{i}^{i(1)}-3 h_{\|}^{(1)}\right)\right], \\
\kappa_{3}^{(1)}=-\frac{\bar{\chi}}{2}\left[\partial_{\perp i} B_{\perp o}^{i(1)}-\partial_{\perp i} v_{\perp o}^{i(1)}+\frac{1}{2} h_{k o}^{j(1)} \partial_{\perp i}\left(n^{k} \mathcal{P}_{j}^{i}\right)\right]=-\frac{1}{4}\left(h_{i o}^{i(1)}-3 h_{\| o}^{(1)}\right)+\left(B_{\| o}^{(1)}-v_{\| o}^{(1)}\right) .
\end{array}
$$

We can finally compute $\Delta \ln a^{(2)}, \Delta x^{0(2)}, \Delta x_{\|}^{(2)}$ and $\Delta x_{\perp}^{i(2)}$. From Eq. 33 we find

$$
\begin{aligned}
& \Delta \ln a^{(2)}=-\delta \nu^{(2)}+E_{\hat{0} \|}^{(2)}-E_{\hat{0} 0}^{(2)}-2 E_{\hat{0} \|}^{(1)}\left(\frac{\mathrm{d} T}{\mathrm{~d} \bar{\chi}}\right)^{(1)}-2 \partial_{\|}\left(E_{\hat{0} \|}^{(1)}-E_{\hat{0} 0}^{(1)}\right) T^{(1)}-\frac{2}{\mathcal{H}}\left(E_{\hat{0} \|}^{(1)}-E_{\hat{0} 0}^{(1)}\right)\left(\frac{\mathrm{d} \Delta \ln a}{\mathrm{~d} \bar{\chi}}\right)^{(1)} \\
& +2\left[-\left(E_{\hat{0} \|}^{(1)}-E_{\hat{0} 0}^{(1)}\right)+\frac{1}{\mathcal{H}}\left(\frac{\mathrm{d} \Delta \ln a}{\mathrm{~d} \bar{\chi}}\right)^{(1)}\right] \delta \nu^{(1)}-2 \delta x^{0(1)}\left(\frac{\mathrm{d} \delta \nu}{\mathrm{d} \bar{\chi}}\right)^{(1)}+2 E_{\hat{0} \perp i}^{(1)} \delta n_{\perp}^{i(1)} \\
& +2\left[\partial_{\perp i}\left(E_{\hat{0} \|}^{(1)}-E_{\hat{0} 0}^{(1)}\right)-\frac{1}{\bar{\chi}} E_{\hat{0} \perp i}^{(1)}\right] \delta x_{\perp}^{i(1)} \\
& =A_{o}^{(2)}-v_{\| o}^{(2)}-\left(A_{o}^{(1)}\right)^{2}+2 A_{o}^{(1)} B_{\| o}^{(1)}-\left(B_{\| o}^{(1)}\right)^{2}-6 A_{o}^{(1)} v_{\| o}^{(1)}+2 B_{\| o}^{(1)} v_{\| o}^{(1)}+v_{k o}^{(1)} v_{o}^{k(1)} \\
& -n^{i} h_{i j o}^{(1)} v_{o}^{j(1)}+2\left(A_{o}^{(1)}-v_{\| o}^{(1)}\right)\left\{-2\left(2 A^{(1)}-B_{\|}^{(1)}\right)+\left(A^{(1)}+v_{\|}^{(1)}-B_{\|}^{(1)}\right)\right. \\
& +\left(\bar{\chi}+\frac{1}{\mathcal{H}}\right) \frac{\mathrm{d}}{\mathrm{d} \bar{\chi}}\left(2 A^{(1)}-B_{\|}^{(1)}\right)-\frac{1}{\mathcal{H}} \frac{\mathrm{d}}{\mathrm{d} \bar{\chi}}\left(A^{(1)}+v_{\|}^{(1)}-B_{\|}^{(1)}\right)+\left(\bar{\chi}+\frac{1}{\mathcal{H}}\right)\left(A^{(1) \prime}-B_{\|}^{(1) \prime}-\frac{1}{2} h_{\|}^{(1) \prime}\right) \\
& \left.+4 I^{(1)}\right\}+2\left(B_{\perp o}^{i(1)}-v_{\perp o}^{i(1)}+\frac{1}{2} n^{k} h_{k o}^{j(1)} \mathcal{P}_{j}^{i}\right)\left\{B_{\perp i}^{(1)}+\bar{\chi} \partial_{\perp i}\left(A^{(1)}+v_{\|}^{(1)}-B_{\|}^{(1)}\right)\right. \\
& \left.-\int_{0}^{\bar{\chi}} \mathrm{d} \tilde{\chi}\left[\tilde{\partial}_{\perp i}\left(2 A^{(1)}-B_{\|}^{(1)}\right)-\left(B_{\perp i}^{(1) \prime}+n^{j} h_{j k}^{(1) \prime} \mathcal{P}_{i}^{k}\right)+\frac{1}{\tilde{\chi}} B_{\perp i}^{(1)}\right]\right\}-A^{(2)}+v_{\|}^{(2)}+7\left(A^{(1)}\right)^{2} \\
& +\left(B_{\|}^{(1)}\right)^{2}-4 A^{(1)} B_{\|}^{(1)}+\left(v_{\|}^{(1)}\right)^{2}+v_{\perp i}^{(1)} v_{\perp}^{i(1)}+v_{\|}^{(1)} h_{\|}^{(1)}-2 A^{(1)} v_{\|}^{(1)}-2 v_{\perp i}^{(1)} B_{\perp}^{i(1)} \\
& -\frac{2}{\mathcal{H}}\left(A^{(1)}-v_{\|}^{(1)}\right)\left[\frac{\mathrm{d}}{\mathrm{d} \bar{\chi}}\left(A^{(1)}-v_{\|}^{(1)}\right)+\left(A^{(1) \prime}-B_{\|}^{(1) \prime}-\frac{1}{2} h_{\|}^{(1) \prime}\right)\right]-4\left[2\left(2 A^{(1)}-B_{\|}^{(1)}\right)\right. \\
& -\left(A^{(1)}+v_{\|}^{(1)}-B_{\|}^{(1)}\right)-\frac{1}{\mathcal{H}} \frac{\mathrm{d}}{\mathrm{d} \bar{\chi}}\left(2 A^{(1)}-B_{\|}^{(1)}\right)+\frac{1}{\mathcal{H}} \frac{\mathrm{d}}{\mathrm{d} \bar{\chi}}\left(A^{(1)}+v_{\|}^{(1)}-B_{\|}^{(1)}\right) \\
& \left.-\frac{1}{\mathcal{H}}\left(A^{(1) \prime}-B_{\|}^{(1) \prime}-\frac{1}{2} h_{\|}^{(1) \prime}\right)\right] I^{(1)}+4 v_{\perp i}^{(1)} S_{\perp}^{i(1)}-2 \partial_{\|}\left(A^{(1)}+v_{\|}^{(1)}-B_{\|}^{(1)}\right) T^{(1)}-2\left[\frac{\mathrm{d}}{\mathrm{d} \bar{\chi}}\left(2 A^{(1)}-B_{\|}^{(1)}\right)\right. \\
& \left.+\left(A^{(1) \prime}-B_{\|}^{(1) \prime}-\frac{1}{2} h_{\|}^{(1) \prime}\right)\right] \int_{0}^{\bar{\chi}} \mathrm{d} \tilde{\chi}\left[2 A^{(1)}-B_{\|}^{(1)}+(\bar{\chi}-\tilde{\chi})\left(A^{(1) \prime}-B_{\|}^{(1) \prime}-\frac{1}{2} h_{\|}^{(1) \prime}\right)\right] \\
& -2\left[\partial_{\perp i}\left(A^{(1)}+v_{\|}^{(1)}-B_{\|}^{(1)}\right)-\frac{1}{\bar{\chi}}\left(v_{\perp i}^{(1)}-B_{\perp i}^{(1)}\right)\right] \int_{0}^{\bar{\chi}} \mathrm{d} \tilde{\chi}\left\{\left(B_{\perp}^{i(1)}+n^{k} h_{k}^{j(1)} \mathcal{P}_{j}^{i}\right)\right. \\
& \left.+(\bar{\chi}-\tilde{\chi})\left[\tilde{\partial}_{\perp}^{i}\left(A^{(1)}-B_{\|}^{(1)}-\frac{1}{2} h_{\|}^{(1)}\right)+\frac{1}{\tilde{\chi}}\left(B_{\perp}^{i(1)}+n^{k} h_{k j}^{(1)} \mathcal{P}^{i j}\right)\right]\right\}+2 I^{(2)} \\
& +2 \int_{0}^{\bar{\chi}} \mathrm{d} \tilde{\chi}\left\{\left(B_{\|}^{(1)}+h_{\|}^{(1)}-4 I^{(1)}\right)\left(A^{(1) \prime}-B_{\|}^{(1) \prime}-\frac{1}{2} h_{\|}^{(1) \prime}\right)-\left(A^{(1)}-B_{\|}^{(1)}-\frac{1}{2} h_{\|}^{(1)}\right)\right. \\
& \times \frac{\mathrm{d}}{\mathrm{d} \tilde{\chi}}\left(2 A^{(1)}-B_{\|}^{(1)}\right)+\left[\left(B_{\perp}^{i(1)}+n^{j} h_{j k}^{(1)} \mathcal{P}^{i k}\right)-2 S_{\perp}^{i(1)}\right]\left[\tilde{\partial}_{\perp i}\left(2 A^{(1)}-B_{\|}^{(1)}\right)\right. \\
& \left.\left.-\left(B_{\perp i}^{(1) \prime}+n^{j} h_{j k}^{(1) \prime} \mathcal{P}_{i}^{k}\right)+\frac{1}{\tilde{\chi}} B_{\perp i}^{(1)}\right]\right\}+\Delta \ln a_{\text {post-Born }}^{(2)},
\end{aligned}
$$


where

$$
\begin{aligned}
& \Delta \ln a_{\text {post-Born }}^{(2)}=+2\left(2 A_{o}^{(1)}-B_{\| o}^{(1)}\right)\left(3 v_{\| o}^{(1)}+2 h_{\| o}^{(1)}-\frac{1}{2} h_{j o}^{j(1)}\right)+2\left(A_{o}^{(1)}-v_{\| o}^{(1)}\right)\left\{2 A^{(1)}-B_{\|}^{(1)}-2 I^{(1)}\right. \\
& \left.-\bar{\chi}\left[\frac{\mathrm{d}}{\mathrm{d} \bar{\chi}}\left(2 A^{(1)}-B_{\|}^{(1)}\right)+\left(A^{(1) \prime}-B_{\|}^{(1) \prime}-\frac{1}{2} h_{\|}^{(1) \prime}\right)\right]\right\}+4\left(-B_{\| o}^{(1)}+v_{\| o}^{(1)}-\frac{3}{4} h_{\| o}^{(1)}+\frac{1}{4} h_{j o}^{j(1)}\right) \\
& \times \int_{0}^{\bar{\chi}} \frac{\mathrm{d} \tilde{\chi}}{\tilde{\chi}}\left(2 A^{(1)}-B_{\|}^{(1)}\right)+2\left(B_{\perp o}^{i(1)}-v_{\perp o}^{i(1)}+\frac{1}{2} n^{k} h_{k o}^{j(1)} \mathcal{P}_{j}^{i}\right)\left\{-\bar{\chi}\left[\partial_{\perp i}\left(2 A^{(1)}-B_{\|}^{(1)}\right)+\frac{1}{\bar{\chi}} B_{\perp i}^{(1)}\right]\right. \\
& \left.+\int_{0}^{\bar{\chi}} \mathrm{d} \tilde{\chi}\left[\tilde{\partial}_{\perp i}\left(2 A^{(1)}-B_{\|}^{(1)}\right)+\frac{1}{\tilde{\chi}} B_{\perp i}^{(1)}\right]\right\}-2\left(2 A^{(1)}-B_{\|}^{(1)}\right)\left(A^{(1)}+\frac{1}{2} h_{\|}^{(1)}-2 I^{(1)}-2 \kappa^{(1)}\right) \\
& +2\left[\frac{\mathrm{d}}{\mathrm{d} \bar{\chi}}\left(2 A^{(1)}-B_{\|}^{(1)}\right)+\left(A^{(1) \prime}-B_{\|}^{(1) \prime}-\frac{1}{2} h_{\|}^{(1) \prime}\right)\right] \int_{0}^{\bar{\chi}} \mathrm{d} \tilde{\chi}\left(A^{(1)}+\frac{1}{2} h_{\|}^{(1)}-2 I^{(1)}\right)+2\left(2 A^{(1) \prime}-B_{\|}^{(1) \prime}\right) T^{(1)} \\
& +2\left[\partial_{\perp i}\left(2 A^{(1)}-B_{\|}^{(1)}\right)+\frac{1}{\bar{\chi}} B_{\perp i}^{(1)}\right] \int_{0}^{\bar{\chi}} \mathrm{d} \tilde{\chi}\left(B_{\perp}^{i(1)}+n^{k} h_{k}^{j(1)} \mathcal{P}_{j}^{i}-2 S_{\perp}^{i(1)}\right) \\
& +2 \int_{0}^{\bar{\chi}} \mathrm{d} \tilde{\chi}\left\{+\left(A^{(1) \prime \prime}-B_{\|}^{(1) \prime \prime}-\frac{1}{2} h_{\|}^{(1) \prime \prime}\right) T^{(1)}+\left(2 A^{(1) \prime}-B_{\|}^{(1) \prime}\right)\left(A^{(1)}-B_{\|}^{(1)}-\frac{1}{2} h_{\|}^{(1)}\right)\right. \\
& -\left(A^{(1) \prime}-B_{\|}^{(1) \prime}-\frac{1}{2} h_{\|}^{(1) \prime}\right)\left(A^{(1)}+\frac{1}{2} h_{\|}^{(1)}-2 I^{(1)}\right)+\left(2 A^{(1)}-B_{\|}^{(1)}\right)\left[\frac{\mathrm{d}}{\mathrm{d} \tilde{\chi}}\left(A^{(1)}+\frac{1}{2} h_{\|}^{(1)}\right)\right. \\
& \left.+\left(A^{(1) \prime}-B_{\|}^{(1) \prime}-\frac{1}{2} h_{\|}^{(1) \prime}\right)\right]-\left(B_{\perp}^{i(1)}+n^{k} h_{k}^{j(1)} \mathcal{P}_{j}^{i}-2 S_{\perp}^{i(1)}\right)\left[\tilde{\partial}_{\perp i}\left(2 A^{(1)}-B_{\|}^{(1)}\right)+\frac{1}{\tilde{\chi}} B_{\perp i}^{(1)}\right] \\
& -\left[\tilde{\partial} \tilde{\perp}_{j}\left(B^{j(1)}+n^{k} h_{k}^{j(1)}\right)\left(2 A^{(1)}-B_{\|}^{(1)}\right)-\frac{2}{\tilde{\chi}}\left(B_{\|}^{(1)}+h_{\|}^{(1)}\right)\left(2 A^{(1)}-B_{\|}^{(1)}\right)-2\left(2 A^{(1)}-B_{\|}^{(1)}\right) \tilde{\partial} \tilde{\nu}_{\perp j} S_{\perp}^{j(1)}\right] \\
& -2\left[\frac{\mathrm{d}}{\mathrm{d} \tilde{\chi}}\left(2 A^{(1)}-B_{\|}^{(1)}\right)-\frac{1}{\tilde{\chi}}\left(2 A^{(1)}-B_{\|}^{(1)}\right)\right] \kappa^{(1)}-\left[\tilde{\partial} \tilde{\perp}_{\perp i}\left(A^{(1) \prime}-B_{\|}^{(1) \prime}-\frac{1}{2} h_{\|}^{(1) \prime}\right)\right. \\
& \left.\left.+\frac{1}{\tilde{\chi}}\left(B_{\perp i}^{(1) \prime}+n^{j} h_{j k}^{(1) \prime} \mathcal{P}_{i}^{k}\right)\right] \delta x_{\perp}^{i(1)}\right\} \cdot
\end{aligned}
$$


Using Eqs 117 and 165, Eq. 377 yields

$$
\begin{aligned}
& \Delta x^{0(2)}=\frac{1}{\mathcal{H}} \Delta \ln a^{(2)}-\frac{\left(\mathcal{H}^{\prime}+\mathcal{H}^{2}\right)}{\mathcal{H}^{3}}\left(\Delta \ln a^{(1)}\right)^{2} \\
& =+\frac{1}{\mathcal{H}} A_{o}^{(2)}-\frac{1}{\mathcal{H}} v_{\| o}^{(2)}-\left(\frac{\mathcal{H}^{\prime}}{\mathcal{H}^{3}}+\frac{2}{\mathcal{H}}\right)\left(A_{o}^{(1)}\right)^{2}-\frac{1}{\mathcal{H}}\left(B_{\| o}^{(1)}\right)^{2}+\frac{2}{\mathcal{H}} A_{o}^{(1)} B_{\| o}^{(1)}+2\left(\frac{\mathcal{H}^{\prime}}{\mathcal{H}^{3}}-\frac{2}{\mathcal{H}}\right) A_{o}^{(1)} v_{\| o}^{(1)} \\
& +\frac{2}{\mathcal{H}} B_{\| o}^{(1)} v_{\| o}^{(1)}-\frac{\mathcal{H}^{\prime}}{\mathcal{H}^{3}}\left(v_{\| o}^{(1)}\right)^{2}+\frac{1}{\mathcal{H}} v_{\perp i o}^{(1)} v_{\perp o}^{i(1)}-\frac{1}{\mathcal{H}} n^{i} h_{i j o}^{(1)} v_{o}^{j(1)}+2\left(A_{o}^{(1)}-v_{\| o}^{(1)}\right) \\
& \times\left\{\left(\frac{\mathcal{H}^{\prime}}{\mathcal{H}^{3}}-\frac{1}{\mathcal{H}}\right)\left(2 A^{(1)}-B_{\|}^{(1)}\right)-\frac{\mathcal{H}^{\prime}}{\mathcal{H}^{3}}\left(A^{(1)}+v_{\|}^{(1)}-B_{\|}^{(1)}\right)+\left(\frac{\bar{\chi}}{\mathcal{H}}+\frac{1}{\mathcal{H}^{2}}\right) \frac{\mathrm{d}}{\mathrm{d} \bar{\chi}}\left(2 A^{(1)}-B_{\|}^{(1)}\right)\right. \\
& \left.-\frac{1}{\mathcal{H}^{2}} \frac{\mathrm{d}}{\mathrm{d} \bar{\chi}}\left(A^{(1)}+v_{\|}^{(1)}-B_{\|}^{(1)}\right)+\left(\frac{\bar{\chi}}{\mathcal{H}}+\frac{1}{\mathcal{H}^{2}}\right)\left(A^{(1) \prime}-B_{\|}^{(1) \prime}-\frac{1}{2} h_{\|}^{(1) \prime}\right)-2\left(\frac{\mathcal{H}^{\prime}}{\mathcal{H}^{3}}-\frac{1}{\mathcal{H}}\right) I^{(1)}\right\} \\
& +2\left(B_{\perp o}^{i(1)}-v_{\perp o}^{i(1)}+\frac{1}{2} n^{k} h_{k o}^{j(1)} \mathcal{P}_{j}^{i}\right)\left\{\frac{1}{\mathcal{H}} B_{\perp i}^{(1)}+\frac{\bar{\chi}}{\mathcal{H}} \partial_{\perp i}\left(A^{(1)}+v_{\|}^{(1)}-B_{\|}^{(1)}\right)\right. \\
& \left.-\frac{1}{\mathcal{H}} \int_{0}^{\bar{\chi}} \mathrm{d} \tilde{\chi}\left[\tilde{\partial}_{\perp i}\left(2 A^{(1)}-B_{\|}^{(1)}\right)-\left(B_{\perp i}^{(1) \prime}+n^{j} h_{j k}^{(1) \prime} \mathcal{P}_{i}^{k}\right)+\frac{1}{\tilde{\chi}} B_{\perp i}^{(1)}\right]\right\}-\frac{1}{\mathcal{H}} A^{(2)}+\frac{1}{\mathcal{H}} v_{\|}^{(2)} \\
& +\left(-\frac{\mathcal{H}^{\prime}}{\mathcal{H}^{3}}+\frac{6}{\mathcal{H}}\right)\left(A^{(1)}\right)^{2}+\frac{1}{\mathcal{H}}\left(B_{\|}^{(1)}\right)^{2}-\frac{4}{\mathcal{H}} A^{(1)} B_{\|}^{(1)}-\frac{\mathcal{H}^{\prime}}{\mathcal{H}^{3}}\left(v_{\|}^{(1)}\right)^{2}+\frac{1}{\mathcal{H}} v_{\perp i}^{(1)} v_{\perp}^{i(1)} \\
& +\frac{1}{\mathcal{H}} v_{\|}^{(1)} h_{\|}^{(1)}+2 \frac{\mathcal{H}^{\prime}}{\mathcal{H}^{3}} A^{(1)} v_{\|}^{(1)}-\frac{2}{\mathcal{H}} v_{\perp i}^{(1)} B_{\perp}^{i(1)}-\frac{2}{\mathcal{H}^{2}}\left(A^{(1)}-v_{\|}^{(1)}\right)\left[\frac{\mathrm{d}}{\mathrm{d} \bar{\chi}}\left(A^{(1)}-v_{\|}^{(1)}\right)\right. \\
& \left.+\left(A^{(1) \prime}-B_{\|}^{(1) \prime}-\frac{1}{2} h_{\|}^{(1) \prime}\right)\right]-4\left[-\left(\frac{\mathcal{H}^{\prime}}{\mathcal{H}^{3}}-\frac{1}{\mathcal{H}}\right)\left(2 A^{(1)}-B_{\|}^{(1)}\right)+\frac{\mathcal{H}^{\prime}}{\mathcal{H}^{3}}\left(A^{(1)}+v_{\|}^{(1)}-B_{\|}^{(1)}\right)\right. \\
& \left.-\frac{1}{\mathcal{H}^{2}} \frac{\mathrm{d}}{\mathrm{d} \bar{\chi}}\left(A^{(1)}-v_{\|}^{(1)}\right)-\frac{1}{\mathcal{H}^{2}}\left(A^{(1) \prime}-B_{\|}^{(1) \prime}-\frac{1}{2} h_{\|}^{(1) \prime}\right)+\left(\frac{\mathcal{H}^{\prime}}{\mathcal{H}^{3}}+\frac{1}{\mathcal{H}}\right) I^{(1)}\right] I^{(1)} \\
& +\frac{4}{\mathcal{H}} v_{\perp i}^{(1)} S_{\perp}^{i(1)}-\frac{2}{\mathcal{H}} \partial_{\|}\left(A^{(1)}+v_{\|}^{(1)}-B_{\|}^{(1)}\right) T^{(1)}-\frac{2}{\mathcal{H}}\left[\frac{\mathrm{d}}{\mathrm{d} \bar{\chi}}\left(2 A^{(1)}-B_{\|}^{(1)}\right)+\left(A^{(1) \prime}-B_{\|}^{(1) \prime}-\frac{1}{2} h_{\|}^{(1) \prime}\right)\right] \\
& \times \int_{0}^{\bar{\chi}} \mathrm{d} \tilde{\chi}\left[2 A^{(1)}-B_{\|}^{(1)}+(\bar{\chi}-\tilde{\chi})\left(A^{(1) \prime}-B_{\|}^{(1) \prime}-\frac{1}{2} h_{\|}^{(1) \prime}\right)\right]-\frac{2}{\mathcal{H}}\left[\partial_{\perp i}\left(A^{(1)}+v_{\|}^{(1)}-B_{\|}^{(1)}\right)\right. \\
& \left.-\frac{1}{\bar{\chi}}\left(v_{\perp i}^{(1)}-B_{\perp i}^{(1)}\right)\right] \int_{0}^{\bar{\chi}} \mathrm{d} \tilde{\chi}\left\{\left(B_{\perp}^{i(1)}+n^{k} h_{k}^{j(1)} \mathcal{P}_{j}^{i}\right)+(\bar{\chi}-\tilde{\chi})\left[\tilde{\partial}_{\perp}^{i}\left(A^{(1)}-B_{\|}^{(1)}-\frac{1}{2} h_{\|}^{(1)}\right)\right.\right. \\
& \left.\left.+\frac{1}{\tilde{\chi}}\left(B_{\perp}^{i(1)}+n^{k} h_{k j}^{(1)} \mathcal{P}^{i j}\right)\right]\right\}+\frac{2}{\mathcal{H}} I^{(2)}+\frac{2}{\mathcal{H}} \int_{0}^{\bar{\chi}} \mathrm{d} \tilde{\chi}\left\{\left(B_{\|}^{(1)}+h_{\|}^{(1)}-4 I^{(1)}\right)\left(A^{(1) \prime}-B_{\|}^{(1) \prime}-\frac{1}{2} h_{\|}^{(1) \prime}\right)\right. \\
& -\left(A^{(1)}-B_{\|}^{(1)}-\frac{1}{2} h_{\|}^{(1)}\right) \frac{\mathrm{d}}{\mathrm{d} \tilde{\chi}}\left(2 A^{(1)}-B_{\|}^{(1)}\right)+\left[\left(B_{\perp}^{i(1)}+n^{j} h_{j k}^{(1)} \mathcal{P}^{i k}\right)-2 S_{\perp}^{i(1)}\right] \\
& \left.\times\left[\tilde{\partial}_{\perp i}\left(2 A^{(1)}-B_{\|}^{(1)}\right)-\left(B_{\perp i}^{(1) \prime}+n^{j} h_{j k}^{(1) \prime} \mathcal{P}_{i}^{k}\right)+\frac{1}{\tilde{\chi}} B_{\perp i}^{(1)}\right]\right\}+\Delta x_{\text {post-Born }}^{0(2)},
\end{aligned}
$$


where

$$
\begin{aligned}
& \Delta x_{\text {post-Born }}^{0(2)}=+\frac{2}{\mathcal{H}}\left(2 A_{o}^{(1)}-B_{\| o}^{(1)}\right)\left(3 v_{\| o}^{(1)}+2 h_{\| o}^{(1)}-\frac{1}{2} h_{j o}^{j(1)}\right)+\frac{2}{\mathcal{H}}\left(A_{o}^{(1)}-v_{\| o}^{(1)}\right)\left\{2 A^{(1)}-B_{\|}^{(1)}-2 I^{(1)}\right. \\
& \left.-\bar{\chi}\left[\frac{\mathrm{d}}{\mathrm{d} \bar{\chi}}\left(2 A^{(1)}-B_{\|}^{(1)}\right)+\left(A^{(1) \prime}-B_{\|}^{(1) \prime}-\frac{1}{2} h_{\|}^{(1) \prime}\right)\right]\right\}+\frac{4}{\mathcal{H}}\left(-B_{\| o}^{(1)}+v_{\| o}^{(1)}-\frac{3}{4} h_{\| o}^{(1)}+\frac{1}{4} h_{j o}^{j(1)}\right) \\
& \times \int_{0}^{\bar{\chi}} \frac{\mathrm{d} \tilde{\chi}}{\tilde{\chi}}\left(2 A^{(1)}-B_{\|}^{(1)}\right)+\frac{2}{\mathcal{H}}\left(B_{\perp o}^{i(1)}-v_{\perp o}^{i(1)}+\frac{1}{2} n^{k} h_{k o}^{j(1)} \mathcal{P}_{j}^{i}\right)\left\{-\bar{\chi}\left[\partial_{\perp i}\left(2 A^{(1)}-B_{\|}^{(1)}\right)+\frac{1}{\bar{\chi}} B_{\perp i}^{(1)}\right]\right. \\
& \left.+\int_{0}^{\bar{\chi}} \mathrm{d} \tilde{\chi}\left[\tilde{\partial}_{\perp i}\left(2 A^{(1)}-B_{\|}^{(1)}\right)+\frac{1}{\tilde{\chi}} B_{\perp i}^{(1)}\right]\right\}-\frac{2}{\mathcal{H}}\left(2 A^{(1)}-B_{\|}^{(1)}\right)\left(A^{(1)}+\frac{1}{2} h_{\|}^{(1)}-2 I^{(1)}-2 \kappa^{(1)}\right) \\
& +\frac{2}{\mathcal{H}}\left[\frac{\mathrm{d}}{\mathrm{d} \bar{\chi}}\left(2 A^{(1)}-B_{\|}^{(1)}\right)+\left(A^{(1) \prime}-B_{\|}^{(1) \prime}-\frac{1}{2} h_{\|}^{(1) \prime}\right)\right] \int_{0}^{\bar{\chi}} \mathrm{d} \tilde{\chi}\left(A^{(1)}+\frac{1}{2} h_{\|}^{(1)}-2 I^{(1)}\right)+\frac{2}{\mathcal{H}}\left(2 A^{(1) \prime}-B_{\|}^{(1) \prime}\right) T^{(1)} \\
& +\frac{2}{\mathcal{H}}\left[\partial_{\perp i}\left(2 A^{(1)}-B_{\|}^{(1)}\right)+\frac{1}{\bar{\chi}} B_{\perp i}^{(1)}\right] \int_{0}^{\bar{\chi}} \mathrm{d} \tilde{\chi}\left(B_{\perp}^{i(1)}+n^{k} h_{k}^{j(1)} \mathcal{P}_{j}^{i}-2 S_{\perp}^{i(1)}\right) \\
& +\frac{2}{\mathcal{H}} \int_{0}^{\bar{\chi}} \mathrm{d} \tilde{\chi}\left\{\left(A^{(1) \prime \prime}-B_{\|}^{(1) \prime \prime}-\frac{1}{2} h_{\|}^{(1) \prime \prime}\right) T^{(1)}+\left(2 A^{(1) \prime}-B_{\|}^{(1) \prime}\right)\left(A^{(1)}-B_{\|}^{(1)}-\frac{1}{2} h_{\|}^{(1)}\right)\right. \\
& -\left(A^{(1) \prime}-B_{\|}^{(1) \prime}-\frac{1}{2} h_{\|}^{(1) \prime}\right)\left(A^{(1)}+\frac{1}{2} h_{\|}^{(1)}-2 I^{(1)}\right)+\left(2 A^{(1)}-B_{\|}^{(1)}\right)\left[\frac{\mathrm{d}}{\mathrm{d} \bar{\chi}}\left(A^{(1)}+\frac{1}{2} h_{\|}^{(1)}\right)\right. \\
& \left.-\left(A^{(1) \prime}-B_{\|}^{(1) \prime}-\frac{1}{2} h_{\|}^{(1) \prime}\right)\right]-\left(B_{\perp}^{i(1)}+n^{k} h_{k}^{j(1)} \mathcal{P}_{j}^{i}-2 S_{\perp}^{i(1)}\right)\left[\tilde{\partial}_{\perp i}\left(2 A^{(1)}-B_{\|}^{(1)}\right)+\frac{1}{\tilde{\chi}} B_{\perp i}^{(1)}\right] \\
& -\left[\tilde{\partial} \perp j\left(B^{j(1)}+n^{k} h_{k}^{j(1)}\right)\left(2 A^{(1)}-B_{\|}^{(1)}\right)-\frac{2}{\tilde{\chi}}\left(B_{\|}^{(1)}+h_{\|}^{(1)}\right)\left(2 A^{(1)}-B_{\|}^{(1)}\right)-2\left(2 A^{(1)}-B_{\|}^{(1)}\right) \tilde{\partial} \tilde{\nu}_{\perp j} S_{\perp}^{j(1)}\right] \\
& -2\left[\frac{\mathrm{d}}{\mathrm{d} \tilde{\chi}}\left(2 A^{(1)}-B_{\|}^{(1)}\right)-\frac{1}{\tilde{\chi}}\left(2 A^{(1)}-B_{\|}^{(1)}\right)\right] \kappa^{(1)}-\left[\tilde{\partial}_{\perp i}\left(A^{(1) \prime}-B_{\|}^{(1) \prime}-\frac{1}{2} h_{\|}^{(1) \prime}\right)\right. \\
& \left.\left.+\frac{1}{\tilde{\chi}}\left(B_{\perp i}^{(1) \prime}+n^{j} h_{j k}^{(1) \prime} \mathcal{P}_{i}^{k}\right)\right] \delta x_{\perp}^{i(1)}\right\} \cdot
\end{aligned}
$$


From Eqs. (71) and 116 we deduce

$$
\begin{aligned}
& \Delta x_{\|}^{(2)}= \delta x^{0(2)}+\delta x_{\|}^{(2)}-\frac{1}{\mathcal{H}} \Delta \ln a^{(2)}+\left(\frac{\mathcal{H}^{\prime}}{\mathcal{H}^{3}}+\frac{1}{\mathcal{H}}\right)\left(\Delta \ln a^{(1)}\right)^{2}-2\left(\frac{\mathrm{d} T}{\mathrm{~d} \bar{\chi}}\right)^{(1)} \delta \chi^{(1)} \\
&= \bar{\chi}\left[\left(A_{o}^{(1)}\right)^{2}-2 A_{o}^{(1)} B_{\| o}^{(1)}+\left(B_{\| o}^{(1)}\right)^{2}+2 A_{o}^{(1)} v_{\| o}^{(1)}-2 B_{\| o}^{(1)} v_{\| o}^{(1)}-v_{\| o}^{(1)} h_{\| o}^{(1)}-\frac{1}{4}\left(h_{\| o}^{(1)}\right)^{2}-v_{\perp k o}^{(1)} v_{\perp o}^{k(1)}\right. \\
&\left.+n^{i} h_{i k o}^{(1)} \mathcal{P}_{j}^{k} v_{o}^{j(1)}-n^{i} h_{i k o}^{(1)} \mathcal{P}_{j}^{k} B_{o}^{j(1)}-\frac{1}{4} n^{i} h_{i j o}^{(1)} \mathcal{P}_{k}^{j} h_{p o}^{k(1)} n^{p}-B_{\perp o}^{i(1)} B_{\perp i o}^{(1)}+2 v_{\perp o}^{i(1)} B_{\perp i o}^{(1)}\right] \\
&-2\left(A_{o}^{(1)}-v_{\| o}^{(1)}\right)\left[\bar{\chi}\left(A^{(1)}-B_{\|}^{(1)}-\frac{1}{2} h_{\|}^{(1)}\right)+2 T^{(1)}\right]+4\left(B_{\perp o}^{i(1)}-v_{\perp o}^{i(1)}+\frac{1}{2} n^{k} h_{k o}^{j(1)} \mathcal{P}_{j}^{i}\right) \\
& \times \int_{0}^{\bar{\chi}} \mathrm{d} \tilde{\chi}(\bar{\chi}-\tilde{\chi})\left[\tilde{\partial}_{\perp}^{i}\left(A^{(1)}-B_{\|}^{(1)}-\frac{1}{2} h_{\|}^{(1)}\right)+\frac{1}{\tilde{\chi}}\left(B_{\perp}^{i(1)}+n^{k} h_{k j}^{(1)} \mathcal{P}^{i j}\right)\right] \\
&+2\left(A^{(1)}-B_{\|}^{(1)}-\frac{1}{2} h_{\|}^{(1)}\right) \int_{0}^{\bar{\chi}} \mathrm{d} \tilde{\chi}\left[2 A^{(1)}-B_{\|}^{(1)}+(\bar{\chi}-\tilde{\chi})\left(A^{(1) \prime}-B_{\|}^{(1) \prime}-\frac{1}{2} h_{\|}^{(1) \prime}\right)\right] \\
&-T^{(2)}+2 \int_{0}^{\bar{\chi}} \mathrm{d} \tilde{\chi}\left[-\left(2 A^{(1)}-B_{\|}^{(1)}\right)^{2}+\frac{1}{2}\left(B_{\|}^{(1)}+h_{\|}^{(1)}\right)\left(2 A^{(1)}+h_{\|}^{(1)}\right)\right. \\
&\left.+\frac{1}{2}\left(B_{\perp}^{i(1)}+\mathcal{P}_{j}^{i} h_{k}^{j(1)} n^{k}\right)\left(B_{i \perp}^{(1)}+n^{p} h_{p m}^{(1)} \mathcal{P}_{i}^{m}\right)+4\left(A^{(1)}-B_{\|}^{(1)}-\frac{1}{2} h_{\|}^{(1)}\right) I^{(1)}-4 S_{\perp}^{i(1)} S_{\perp}^{j(1)} \delta_{i j}\right] \\
&+2 \int_{0}^{\bar{\chi}} \mathrm{d} \tilde{\chi}(\bar{\chi}-\tilde{\chi})\left\{\left(A^{(1)}-B_{\|}^{(1)}-\frac{1}{2} h_{\|}^{(1)}\right)\left[2\left(A^{(1) \prime}-B_{\|}^{(1) \prime}-\frac{1}{2} h_{\|}^{(1) \prime}\right)+\frac{\mathrm{d}}{\mathrm{d} \tilde{\chi}}\left(2 A^{(1)}-B_{\|}^{(1)}\right)\right]\right. \\
&\left.-\left(B_{\perp}^{i(1)}+n^{k} h_{k}^{j(1)} \mathcal{P}_{j}^{i}\right)\left[\tilde{\partial}_{\perp i}\left(A^{(1)}-B_{\|}^{(1)}-\frac{1}{2} h_{\|}^{(1)}\right)+\frac{1}{\tilde{\chi}}\left(B_{\perp i}^{(1)}+n^{m} h_{m p}^{(1)} \mathcal{P}_{i}^{p}\right)\right]\right\} \\
&-\frac{2}{\mathcal{H}}\left(A^{(1)}-B_{\|}^{(1)}-\frac{1}{2} h_{\|}^{(1)}\right) \Delta \ln a^{(1)}-\frac{1}{\mathcal{H}} \Delta \ln a^{(2)}+\left(\frac{\mathcal{H}^{\prime}}{\mathcal{H}^{3}}+\frac{1}{\mathcal{H}}\right)\left(\Delta \ln a^{(1)}\right)^{2} \\
&+\left(\delta x^{0(2)}+\delta x_{\|}^{(2)}\right) \\
& \operatorname{post}-\operatorname{Born}
\end{aligned}
$$


and from Eqs. 72 and 115 we find

$$
\begin{aligned}
& \Delta x_{\perp}^{i(2)}=\delta x_{\perp}^{i(2)}+2 \delta n_{\perp}^{i(1)} \delta x^{0(1)}-\frac{2}{\mathcal{H}} \delta n_{\perp}^{i(1)} \Delta \ln a^{(1)} \\
& =\bar{\chi}\left[B_{\perp o}^{i(2)}-v_{\perp o}^{i(2)}+\frac{1}{2} n^{j} h_{j k o}^{(2)} \mathcal{P}^{k i}-2 A_{o}^{(1)} B_{\perp o}^{i(1)}+2 A_{o}^{(1)} v_{\perp o}^{i(1)}+A_{o}^{(1)} n^{k} h_{k o}^{j(1)} \mathcal{P}_{j}^{i}-v_{\| o}^{(1)} v_{\perp o}^{i(1)}\right. \\
& -v_{\| o}^{(1)} B_{\perp o}^{i(1)}+B_{\| o}^{(1)} B_{\perp o}^{i(1)}-B_{\| o}^{(1)} v_{\perp o}^{i(1)}-3 v_{\| o}^{(1)} n^{k} h_{k o}^{j(1)} \mathcal{P}_{j}^{i}-2 v_{\perp o}^{j(1)} \mathcal{P}_{j}^{l} h_{l o}^{k(1)} \mathcal{P}_{k}^{i}-\frac{1}{4} h_{\| o}^{(1)} n^{j} h_{j k o}^{(1)} \mathcal{P}^{k i} \\
& \left.-\frac{1}{4} n^{j} h_{j o}^{k(1)} \mathcal{P}_{k}^{l} h_{l o}^{p(1)} \mathcal{P}_{p}^{i(1)}\right]+2 \bar{\chi}\left(A_{o}^{(1)}-v_{\| o}^{(1)}\right)\left[\left(B_{\perp}^{i(1)}+n^{k} h_{k}^{j(1)} \mathcal{P}_{j}^{i}\right)-2 S_{\perp}^{i(1)}\right] \\
& -4\left(A_{o}^{(1)}-v_{\| o}^{(1)}\right) \int_{0}^{\bar{\chi}} \mathrm{d} \tilde{\chi}\left\{\left(B_{\perp}^{i(1)}+n^{k} h_{k}^{j(1)} \mathcal{P}_{j}^{i}\right)+(\bar{\chi}-\tilde{\chi})\left[\tilde{\partial}_{\perp}^{i}\left(A^{(1)}-B_{\|}^{(1)}-\frac{1}{2} h_{\|}^{(1)}\right)\right.\right. \\
& \left.\left.+\frac{1}{\tilde{\chi}}\left(B_{\perp}^{i(1)}+n^{k} h_{k j}^{(1)} \mathcal{P}^{i j}\right)\right]\right\}+2\left(B_{\perp j o}^{(1)}-v_{\perp j o}^{(1)}+\frac{1}{2} n^{k} h_{k o}^{l(1)} \mathcal{P}_{j l}\right) \int_{0}^{\bar{\chi}} \mathrm{d} \tilde{\chi}\left\{-\mathcal{P}^{j m} h_{m}^{p(1)} \mathcal{P}_{p}^{i}\right. \\
& \left.+2(\bar{\chi}-\tilde{\chi})\left[\tilde{\partial}_{\perp}^{[i} B_{\perp}^{j](1)}+\tilde{\partial}_{\perp}^{[i}\left(\mathcal{P}_{m}^{j]} h_{q}^{m(1)} n^{q}\right)-\frac{1}{\tilde{\chi}}\left(n^{[i} B_{\perp}^{j](1)}+n^{[i} \mathcal{P}_{m}^{j]} h_{q}^{m(1)} n^{q}\right)\right]\right\} \\
& +2\left(B_{\perp o}^{i(1)}-v_{\perp o}^{i(1)}+\frac{1}{2} n^{k} h_{k o}^{j(1)} \mathcal{P}_{j}^{i}\right)\left\{\int_{0}^{\bar{\chi}} \mathrm{d} \tilde{\chi}\left[2 A^{(1)}-B_{\|}^{(1)}+(\bar{\chi}-\tilde{\chi})\left(A^{(1) \prime}-B_{\|}^{(1) \prime}-\frac{1}{2} h_{\|}^{(1) \prime}\right)\right]\right. \\
& \left.-\frac{1}{\mathcal{H}} \Delta \ln a^{(1)}\right\}+\frac{2}{\mathcal{H}}\left(B_{\perp}^{i(1)}+n^{k} h_{k}^{j(1)} \mathcal{P}_{j}^{i}-2 S_{\perp}^{i(1)}\right) \Delta \ln a^{(1)} \\
& -2\left[\left(B_{\perp}^{i(1)}+n^{k} h_{k}^{j(1)} \mathcal{P}_{j}^{i}\right)-2 S_{\perp}^{i(1)}\right] \int_{0}^{\bar{\chi}} \mathrm{d} \tilde{\chi}\left[2 A^{(1)}-B_{\|}^{(1)}+(\bar{\chi}-\tilde{\chi})\left(A^{(1) \prime}-B_{\|}^{(1) \prime}-\frac{1}{2} h_{\|}^{(1) \prime}\right)\right] \\
& +\int_{0}^{\bar{\chi}} \mathrm{d} \tilde{\chi}\left\{-B_{\perp}^{i(2)}-n^{j} h_{j k}^{(2)} \mathcal{P}^{k i}+4 A^{(1)} B_{\perp}^{i(1)}-2 B_{\|}^{(1)} B_{\perp}^{i(1)}+2 A^{(1)} n^{j} h_{j k}^{(1)} \mathcal{P}^{k i}+h_{\|}^{(1)} n^{j} h_{j k}^{(1)} \mathcal{P}^{k i}\right. \\
& \left.+2\left(B_{\perp}^{j(1)}+n^{k} h_{k}^{p(1)} \mathcal{P}_{p}^{j}\right) \mathcal{P}_{j}^{l} h_{l}^{k(1)} \mathcal{P}_{k}^{i(1)}-8\left(B_{\perp}^{i(1)}+n^{j} h_{j k}^{(1)} \mathcal{P}^{i k}\right) I^{(1)}-4 \mathcal{P}_{j}^{l} h_{l}^{k(1)} \mathcal{P}_{k}^{i} S_{\perp}^{j(1)}\right\} \\
& +\int_{0}^{\bar{\chi}} \mathrm{d} \tilde{\chi}(\bar{\chi}-\tilde{\chi})\left\{-\left[\tilde{\partial}_{\perp}^{i}\left(A^{(2)}-B_{\|}^{(2)}-\frac{1}{2} h_{\|}^{(2)}\right)+\frac{1}{\tilde{\chi}}\left(B_{\perp}^{i(2)}+n^{k} h_{k j}^{(2)} \mathcal{P}^{i j}\right)\right]\right. \\
& -2\left(A^{(1) \prime}-B_{\|}^{(1) \prime}-\frac{1}{2} h_{\|}^{(1) \prime}\right)\left(B_{\perp}^{i(1)}+n^{j} h_{j k}^{(1)} \mathcal{P}^{i k}\right)+2\left(2 A^{(1)}-B_{\|}^{(1)}\right) \frac{\mathrm{d}}{\mathrm{d} \tilde{\chi}}\left(B_{\perp}^{i(1)}+n^{j} h_{j k}^{(1)} \mathcal{P}^{i k}\right) \\
& +4\left(2 A^{(1)}-B_{\|}^{(1)}-2 I^{(1)}\right)\left[\tilde{\partial}_{\perp}^{i}\left(A^{(1)}-B_{\|}^{(1)}-\frac{1}{2} h_{\|}^{(1)}\right)+\frac{1}{\tilde{\chi}}\left(B_{\perp}^{i(1)}+n^{k} h_{k j}^{(1)} \mathcal{P}^{i j}\right)\right] \\
& -4\left[\left(B_{\perp j}^{(1)}+n^{p} h_{p}^{k(1)} \mathcal{P}_{j k}\right)-2 \delta_{j p} S_{\perp}^{p(1)}\right]\left[\tilde{\partial}_{\perp}^{[i} B_{\perp}^{j](1)}+\tilde{\partial}_{\perp}^{[i}\left(\mathcal{P}_{m}^{j]} h_{q}^{m(1)} n^{q}\right)-\frac{1}{\tilde{\chi}}\left(n^{[i} B_{\perp}^{j](1)}+n^{[i} \mathcal{P}_{m}^{j]} h_{q}^{m(1)} n^{q}\right)\right] \\
& \left.+2\left(A^{(1)}-B_{\|}^{(1)}-\frac{1}{2} h_{\|}^{(1)}\right)\left[\tilde{\partial}_{\perp}^{i}\left(B_{\|}^{(1)}+h_{\|}^{(1)}\right)-\partial_{\|}\left(B_{\perp}^{i(1)}+n^{p} h_{p q}^{(1)} \mathcal{P}^{i q}\right)-\frac{1}{\tilde{\chi}}\left(B_{\perp}^{i(1)}+2 n^{p} h_{p q}^{(1)} \mathcal{P}^{i q}\right)\right]\right\} \\
& +\delta x_{\perp \text { post-Born }}^{i(2)} \text {. }
\end{aligned}
$$

The next step is to compute $\partial_{\|} \Delta x_{\|}^{(2)}$ and $\kappa^{(2)}$. From Eq. $\sqrt{79}$ we have $\partial_{\|} \Delta x_{\|}^{(2)}(\eta, \overline{\mathbf{x}})=\partial_{\bar{\chi}} \Delta x_{\|}^{(2)}(\bar{\chi}, \mathbf{n})$, so that

$$
\begin{aligned}
\partial_{\|} \Delta x_{\|}^{(2)}= & \delta \nu^{(2)}+\delta n_{\|}^{(2)}-\frac{\mathrm{d}}{\mathrm{d} \bar{\chi}}\left(\frac{1}{\mathcal{H}} \Delta \ln a^{(2)}\right)+\frac{\mathrm{d}}{\mathrm{d} \bar{\chi}}\left[\left(\frac{\mathcal{H}^{\prime}}{\mathcal{H}^{3}}+\frac{1}{\mathcal{H}}\right)\left(\Delta \ln a^{(1)}\right)^{2}\right]-2 \frac{\mathrm{d}}{\mathrm{d} \bar{\chi}}\left[\left(\frac{\mathrm{d} T}{\mathrm{~d} \bar{\chi}}\right)^{(1)} \delta \chi^{(1)}\right] \\
= & -\frac{\mathcal{H}^{\prime}}{\mathcal{H}^{2}} \Delta \ln a^{(2)}+\left[-\frac{\mathcal{H}^{\prime \prime}}{\mathcal{H}^{3}}+3\left(\frac{\mathcal{H}^{\prime}}{\mathcal{H}^{2}}\right)^{2}+\frac{\mathcal{H}^{\prime}}{\mathcal{H}^{2}}\right]\left(\Delta \ln a^{(1)}\right)^{2}+2\left[\frac{\mathcal{H}^{\prime}}{\mathcal{H}^{2}}\left(\frac{\mathrm{d} T}{\mathrm{~d} \bar{\chi}}\right)^{(1)}+\frac{1}{\mathcal{H}}\left(\frac{\mathrm{d}^{2} T}{\mathrm{~d} \bar{\chi}^{2}}\right)^{(1)}\right] \Delta \ln a^{(1)} \\
& +2\left(\frac{\mathcal{H}^{\prime}}{\mathcal{H}^{3}}+\frac{1}{\mathcal{H}}\right) \Delta \ln a^{(1)}\left(\frac{\mathrm{d} \Delta \ln a}{\mathrm{~d} \bar{\chi}}\right)^{(1)}-\frac{1}{\mathcal{H}}\left(\frac{\mathrm{d} \Delta \ln a}{\mathrm{~d} \bar{\chi}}\right)^{(2)}-2\left(\frac{\mathrm{d}^{2} T}{\mathrm{~d} \bar{\chi}^{2}}\right)^{(1)} \delta x^{0(1)}-2\left(\frac{\mathrm{d} T}{\mathrm{~d} \bar{\chi}}\right)^{(1)} \delta \nu^{(1)} \\
& +\frac{2}{\mathcal{H}}\left(\frac{\mathrm{d} T}{\mathrm{~d} \bar{\chi}}\right)^{(1)}\left(\frac{\mathrm{d} \Delta \ln a}{\mathrm{~d} \bar{\chi}}\right)^{(1)}+\delta \nu^{(2)}+\delta n_{\|}^{(2)}
\end{aligned}
$$


To obtain explicitly Eq. 132 we have to determine

$$
\begin{aligned}
& \left(\frac{\mathrm{d} \Delta \ln a}{\mathrm{~d} \bar{\chi}}\right)^{(2)}=+2\left(A_{o}^{(1)}-v_{\| o}^{(1)}\right)\left\{\left(\frac{\mathcal{H}^{\prime}}{\mathcal{H}^{2}}-1\right)\left[\frac{\mathrm{d}}{\mathrm{d} \bar{\chi}}\left(A^{(1)}-v_{\|}^{(1)}\right)+\left(A^{(1) \prime}-B_{\|}^{(1) \prime}-\frac{1}{2} h_{\|}^{(1) \prime}\right)\right]\right. \\
& +\frac{1}{\mathcal{H}} \frac{\mathrm{d}}{\mathrm{d} \bar{\chi}}\left[\frac{\mathrm{d}}{\mathrm{d} \bar{\chi}}\left(A^{(1)}-v_{\|}^{(1)}\right)+\left(A^{(1) \prime}-B_{\|}^{(1) \prime}-\frac{1}{2} h_{\|}^{(1) \prime}\right)\right]+\bar{\chi} \frac{\mathrm{d}}{\mathrm{d} \bar{\chi}}\left[\frac{\mathrm{d}}{\mathrm{d} \bar{\chi}}\left(2 A^{(1)}-B_{\|}^{(1)}\right)\right. \\
& \left.\left.+\left(A^{(1) \prime}-B_{\|}^{(1) \prime}-\frac{1}{2} h_{\|}^{(1) \prime}\right)\right]\right\}+2\left(B_{\perp o}^{i(1)}-v_{\perp o}^{i(1)}+\frac{1}{2} n^{k} h_{k o}^{j(1)} \mathcal{P}_{j}^{i}\right)\left[\frac{\mathrm{d}}{\mathrm{d} \bar{\chi}} B_{\perp i}^{(1)}-\partial_{\perp i}\left(A^{(1)}-v_{\|}^{(1)}\right)\right. \\
& \left.+\bar{\chi} \frac{\mathrm{d}}{\mathrm{d} \bar{\chi}} \partial_{\perp i}\left(A^{(1)}+v_{\|}^{(1)}-B_{\|}^{(1)}\right)+\left(B_{\perp i}^{(1) \prime}+n^{j} h_{j k}^{(1) \prime} \mathcal{P}_{i}^{k}\right)-\frac{1}{\tilde{\chi}} B_{\perp i}^{(1)}\right]+\frac{\mathrm{d}}{\mathrm{d} \bar{\chi}}\left(-A^{(2)}+v_{\|}^{(2)}\right) \\
& -\left(A^{(2) \prime}-B_{\|}^{(2) \prime}-\frac{1}{2} h_{\|}^{(2) \prime}\right)+\frac{\mathrm{d}}{\mathrm{d} \bar{\chi}}\left[7\left(A^{(1)}\right)^{2}+\left(B_{\|}^{(1)}\right)^{2}-4 A^{(1)} B_{\|}^{(1)}-2 A^{(1)} v_{\|}^{(1)}\right. \\
& \left.+\left(v_{\|}^{(1)}\right)^{2}+v_{\|}^{(1)} h_{\|}^{(1)}\right]-2\left[\frac{\mathrm{d}}{\mathrm{d} \bar{\chi}}\left(2 A^{(1)}-B_{\|}^{(1)}\right)+\left(A^{(1) \prime}-B_{\|}^{(1) \prime}-\frac{1}{2} h_{\|}^{(1) \prime}\right)\right]\left(2 A^{(1)}-B_{\|}^{(1)}\right) \\
& -2\left\{\frac{\mathcal{H}^{\prime}}{\mathcal{H}^{2}}\left(A^{(1)}-v_{\|}^{(1)}\right)+\frac{1}{\mathcal{H}}\left[\frac{\mathrm{d}}{\mathrm{d} \bar{\chi}}\left(A^{(1)}-v_{\|}^{(1)}\right)+\left(A^{(1) \prime}-B_{\|}^{(1) \prime}-\frac{1}{2} h_{\|}^{(1) \prime}\right)\right]\right\}\left[\frac{\mathrm{d}}{\mathrm{d} \bar{\chi}}\left(A^{(1)}-v_{\|}^{(1)}\right)\right. \\
& \left.+\left(A^{(1) \prime}-B_{\|}^{(1) \prime}-\frac{1}{2} h_{\|}^{(1) \prime}\right)\right]+2\left[\left(2 A^{(1)}+h_{\|}^{(1)}\right)+\left(A^{(1)}-v_{\|}^{(1)}\right)\right]\left(A^{(1) \prime}-B_{\|}^{(1) \prime}-\frac{1}{2} h_{\|}^{(1) \prime}\right) \\
& -\frac{2}{\mathcal{H}}\left(A^{(1)}-v_{\|}^{(1)}\right) \frac{\mathrm{d}}{\mathrm{d} \bar{\chi}}\left[\frac{\mathrm{d}}{\mathrm{d} \bar{\chi}}\left(A^{(1)}-v_{\|}^{(1)}\right)+\left(A^{(1) \prime}-B_{\|}^{(1) \prime}-\frac{1}{2} h_{\|}^{(1) \prime}\right)\right]+2\left(A^{(1)}-B_{\|}^{(1)}-\frac{1}{2} h_{\|}^{(1)}\right) \\
& \times\left[-\frac{\mathrm{d}}{\mathrm{d} \bar{\chi}}\left(2 A^{(1)}-B_{\|}^{(1)}\right)+\partial_{\|}\left(A^{(1)}+v_{\|}^{(1)}-B_{\|}^{(1)}\right)\right]+2\left(B_{\perp}^{i(1)}+n^{j} h_{j k}^{(1)} \mathcal{P}^{i k}\right)\left[\partial_{\perp i}\left(A^{(1)}-v_{\|}^{(1)}\right)\right. \\
& \left.-\left(B_{\perp i}^{(1) \prime}+n^{j} h_{j k}^{(1) \prime} \mathcal{P}_{i}^{k}\right)\right]-2 v_{\perp i}^{(1)} \partial_{\perp}^{i}\left(A^{(1)}-B_{\|}^{(1)}-\frac{1}{2} h_{\|}^{(1)}\right)+2 \frac{\mathrm{d}}{\mathrm{d} \bar{\chi}}\left[\frac{1}{2} v_{\perp i}^{(1)} v_{\perp}^{i(1)}-v_{\perp i}^{(1)} B_{\perp}^{i(1)}\right] \\
& +4\left\{\left(\frac{\mathcal{H}^{\prime}}{\mathcal{H}^{2}}-1\right)\left[\frac{\mathrm{d}}{\mathrm{d} \bar{\chi}}\left(A^{(1)}-v_{\|}^{(1)}\right)+\left(A^{(1) \prime}-B_{\|}^{(1) \prime}-\frac{1}{2} h_{\|}^{(1) \prime}\right)\right]+\frac{1}{\mathcal{H}} \frac{\mathrm{d}}{\mathrm{d} \bar{\chi}}\left[\frac{\mathrm{d}}{\mathrm{d} \bar{\chi}}\left(A^{(1)}-v_{\|}^{(1)}\right)\right.\right. \\
& \left.\left.+\left(A^{(1) \prime}-B_{\|}^{(1) \prime}-\frac{1}{2} h_{\|}^{(1) \prime}\right)\right]\right\} I^{(1)}-2\left[\frac{\mathrm{d}}{\mathrm{d} \bar{\chi}} \partial_{\|}\left(A^{(1)}+v_{\|}^{(1)}-B_{\|}^{(1)}\right)\right] T^{(1)}-2 \frac{\mathrm{d}}{\mathrm{d} \bar{\chi}}\left[\frac{\mathrm{d}}{\mathrm{d} \bar{\chi}}\left(2 A^{(1)}-B_{\|}^{(1)}\right)\right. \\
& \left.+\left(A^{(1) \prime}-B_{\|}^{(1) \prime}-\frac{1}{2} h_{\|}^{(1) \prime}\right)\right] \int_{0}^{\bar{\chi}} \mathrm{d} \tilde{\chi}\left[2 A^{(1)}-B_{\|}^{(1)}+(\bar{\chi}-\tilde{\chi})\left(A^{(1) \prime}-B_{\|}^{(1) \prime}-\frac{1}{2} h_{\|}^{(1) \prime}\right)\right] \\
& -4\left[\partial_{\perp i}\left(A^{(1)}-v_{\|}^{(1)}\right)-\left(B_{\perp i}^{(1) \prime}+n^{j} h_{j k}^{(1) \prime} \mathcal{P}_{i}^{k}\right)+\frac{1}{\tilde{\chi}} v_{\perp i}^{(1)}-\frac{\mathrm{d}}{\mathrm{d} \bar{\chi}} v_{\perp i}^{(1)}\right] S_{\perp}^{i(1)} \\
& -2 \frac{\mathrm{d}}{\mathrm{d} \bar{\chi}}\left[\partial_{\perp i}\left(A^{(1)}+v_{\|}^{(1)}-B_{\|}^{(1)}\right)-\frac{1}{\bar{\chi}}\left(v_{\perp i}^{(1)}-B_{\perp i}^{(1)}\right)\right] \int_{0}^{\bar{\chi}} \mathrm{d} \tilde{\chi}\left\{\left(B_{\perp}^{i(1)}+n^{k} h_{k}^{j(1)} \mathcal{P}_{j}^{i}\right)\right. \\
& \left.+(\bar{\chi}-\tilde{\chi})\left[\tilde{\partial}_{\perp}^{i}\left(A^{(1)}-B_{\|}^{(1)}-\frac{1}{2} h_{\|}^{(1)}\right)+\frac{1}{\tilde{\chi}}\left(B_{\perp}^{i(1)}+n^{k} h_{k j}^{(1)} \mathcal{P}^{i j}\right)\right]\right\}+\left(\frac{\mathrm{d} \Delta \ln a}{\mathrm{~d} \bar{\chi}}\right)_{\text {post-Born }}^{(2)},
\end{aligned}
$$


where

$$
\begin{aligned}
& \left(\frac{\mathrm{d} \Delta \ln a}{\mathrm{~d} \bar{\chi}}\right)_{\text {post-Born }}^{(2)}=-2 \bar{\chi}\left(A_{o}^{(1)}-v_{\| o}^{(1)}\right) \frac{\mathrm{d}}{\mathrm{d} \bar{\chi}}\left[\frac{\mathrm{d}}{\mathrm{d} \bar{\chi}}\left(2 A^{(1)}-B_{\|}^{(1)}\right)+\left(A^{(1) \prime}-B_{\|}^{(1) \prime}-\frac{1}{2} h_{\|}^{(1) \prime}\right)\right] \\
& -2 \bar{\chi}\left(B_{\perp o}^{i(1)}-v_{\perp o}^{i(1)}+\frac{1}{2} n^{k} h_{k o}^{j(1)} \mathcal{P}_{j}^{i}\right)\left\{\partial_{\perp i} \frac{\mathrm{d}}{\mathrm{d} \bar{\chi}}\left(2 A^{(1)}-B_{\|}^{(1)}\right)+\partial_{\perp i}\left(A^{(1) \prime}-B_{\|}^{(1) \prime}-\frac{1}{2} h_{\|}^{(1) \prime}\right)\right. \\
& \left.-\frac{1}{\bar{\chi}}\left[\partial_{\perp i}\left(2 A^{(1)}-B_{\|}^{(1)}\right)+\left(\frac{1}{\bar{\chi}} B_{\perp i}^{(1)}-\frac{\mathrm{d}}{\mathrm{d} \bar{\chi}} B_{\perp i}^{(1)}\right)-\left(B_{\perp i}^{(1) \prime}+n^{j} h_{j k}^{(1) \prime} \mathcal{P}_{i}^{k}\right)\right]\right\} \\
& +2 \frac{\mathrm{d}}{\mathrm{d} \bar{\chi}}\left[\frac{\mathrm{d}}{\mathrm{d} \bar{\chi}}\left(2 A^{(1)}-B_{\|}^{(1)}\right)+\left(A^{(1) \prime}-B_{\|}^{(1) \prime}-\frac{1}{2} h_{\|}^{(1) \prime}\right)\right] \int_{0}^{\bar{\chi}} \mathrm{d} \tilde{\chi}\left(A^{(1)}+\frac{1}{2} h_{\|}^{(1)}-2 I^{(1)}\right) \\
& +2\left[\frac{\mathrm{d}}{\mathrm{d} \bar{\chi}}\left(2 A^{(1) \prime}-B_{\|}^{(1) \prime}\right)+\left(A^{(1) \prime \prime}-B_{\|}^{(1) \prime \prime}-\frac{1}{2} h_{\|}^{(1) \prime \prime}\right)\right] T^{(1)} \\
& +2\left\{\partial_{\perp i} \frac{\mathrm{d}}{\mathrm{d} \bar{\chi}}\left(2 A^{(1)}-B_{\|}^{(1)}\right)+\partial_{\perp i}\left(A^{(1) \prime}-B_{\|}^{(1) \prime}-\frac{1}{2} h_{\|}^{(1) \prime}\right)-\frac{1}{\bar{\chi}}\left[\partial_{\perp i}\left(2 A^{(1)}-B_{\|}^{(1)}\right)+\left(\frac{1}{\bar{\chi}} B_{\perp i}^{(1)}-\frac{\mathrm{d}}{\mathrm{d} \bar{\chi}} B_{\perp i}^{(1)}\right)\right.\right. \\
& \left.\left.-\left(B_{\perp i}^{(1) \prime}+n^{j} h_{j k}^{(1) \prime} \mathcal{P}_{i}^{k}\right)\right]\right\} \int_{0}^{\bar{\chi}} \mathrm{d} \tilde{\chi}\left(B_{\perp}^{i(1)}+n^{k} h_{k}^{j(1)} \mathcal{P}_{j}^{i}-2 S_{\perp}^{i(1)}\right) \cdot
\end{aligned}
$$


Then we obtain

$$
\begin{aligned}
& \partial_{\|} \Delta x_{\|}^{(2)}=\left(A_{o}^{(1)}\right)^{2}-2 A_{o}^{(1)} B_{\| o}^{(1)}+\left(B_{\| o}^{(1)}\right)^{2}+2 A_{o}^{(1)} v_{\| o}^{(1)}-2 B_{\| o}^{(1)} v_{\| o}^{(1)}-v_{\| o}^{(1)} h_{\| o}^{(1)}-\frac{1}{4}\left(h_{\| o}^{(1)}\right)^{2}-v_{\perp k o}^{(1)} v_{\perp o}^{k(1)} \\
& +n^{i} h_{i k o}^{(1)} \mathcal{P}_{j}^{k} v_{o}^{j(1)}-n^{i} h_{i k o}^{(1)} \mathcal{P}_{j}^{k} B_{o}^{j(1)}-\frac{1}{4} n^{i} h_{i j o}^{(1)} \mathcal{P}_{k}^{j} h_{p o}^{k(1)} n^{p}-B_{\perp o}^{i(1)} B_{\perp i o}^{(1)}+2 v_{\perp o}^{i(1)} B_{\perp i o}^{(1)}+2\left(A_{o}^{(1)}-v_{\| o}^{(1)}\right) \\
& \times\left\{\left(-\frac{\mathcal{H}^{\prime}}{\mathcal{H}^{3}}+\frac{1}{\mathcal{H}}\right)\left[\frac{\mathrm{d}}{\mathrm{d} \bar{\chi}}\left(A^{(1)}-v_{\|}^{(1)}\right)+\left(A^{(1) \prime}-B_{\|}^{(1) \prime}-\frac{1}{2} h_{\|}^{(1) \prime}\right)\right]-\frac{\bar{\chi}}{\mathcal{H}} \frac{\mathrm{d}}{\mathrm{d} \bar{\chi}}\left[\frac{\mathrm{d}}{\mathrm{d} \bar{\chi}}\left(2 A^{(1)}-B_{\|}^{(1)}\right)\right.\right. \\
& \left.+\left(A^{(1) \prime}-B_{\|}^{(1) \prime}-\frac{1}{2} h_{\|}^{(1) \prime}\right)\right]+\left(A^{(1)}-B_{\|}^{(1)}-\frac{1}{2} h_{\|}^{(1)}\right)-\bar{\chi} \frac{\mathrm{d}}{\mathrm{d} \bar{\chi}}\left(A^{(1)}-B_{\|}^{(1)}-\frac{1}{2} h_{\|}^{(1)}\right)-\frac{1}{\mathcal{H}^{2}} \frac{\mathrm{d}}{\mathrm{d} \bar{\chi}}\left[\frac{\mathrm{d}}{\mathrm{d} \bar{\chi}}\left(A^{(1)}-v_{\|}^{(1)}\right)\right. \\
& \left.\left.+\left(A^{(1) \prime}-B_{\|}^{(1) \prime}-\frac{1}{2} h_{\|}^{(1) \prime}\right)\right]\right\}+2\left(B_{\perp o}^{i(1)}-v_{\perp o}^{i(1)}+\frac{1}{2} n^{k} h_{k o}^{j(1)} \mathcal{P}_{j}^{i}\right)\left[-\frac{1}{\mathcal{H}} \frac{\mathrm{d}}{\mathrm{d} \bar{\chi}} B_{\perp i}^{(1)}+\frac{1}{\mathcal{H}} \partial_{\perp i}\left(A^{(1)}-v_{\|}^{(1)}\right)\right. \\
& \left.-\frac{1}{\mathcal{H}}\left(B_{\perp i}^{(1) \prime}+n^{j} h_{j k}^{(1) \prime} \mathcal{P}_{i}^{k}\right)-2 \frac{\bar{\chi}}{\mathcal{H}} \frac{\mathrm{d}}{\mathrm{d} \bar{\chi}} \partial_{\perp i}\left(A^{(1)}+v_{\|}^{(1)}-B_{\|}^{(1)}\right)+\frac{1}{\mathcal{H} \bar{\chi}} B_{\perp i}^{(1)}-4 \delta_{i l} S_{\perp}^{l(1)}\right]+A^{(2)}-B_{\|}^{(2)}-\frac{1}{2} h_{\|}^{(2)} \\
& +\frac{1}{\mathcal{H}}\left[\frac{\mathrm{d}}{\mathrm{d} \bar{\chi}}\left(A^{(2)}-v_{\|}^{(2)}\right)+\left(A^{(2) \prime}-B_{\|}^{(2) \prime}-\frac{1}{2} h_{\|}^{(2) \prime}\right)\right]-2\left(2 A^{(1)}+h_{\|}^{(1)}\right)\left(A^{(1)}-B_{\|}^{(1)}-\frac{1}{2} h_{\|}^{(1)}\right)-\frac{1}{\mathcal{H}} \frac{\mathrm{d}}{\mathrm{d} \bar{\chi}}\left[7\left(A^{(1)}\right)^{2}\right. \\
& \left.+\left(B_{\|}^{(1)}\right)^{2}-4 A^{(1)} B_{\|}^{(1)}-2 A^{(1)} v_{\|}^{(1)}+\left(v_{\|}^{(1)}\right)^{2}+v_{\|}^{(1)} h_{\|}^{(1)}+v_{\perp i}^{(1)} v_{\perp}^{i(1)}-2 v_{\perp i}^{(1)} B_{\perp}^{i(1)}\right]+\frac{2}{\mathcal{H}^{2}}\left(A^{(1)}-v_{\|}^{(1)}\right) \\
& \times \frac{\mathrm{d}}{\mathrm{d} \bar{\chi}}\left[\frac{\mathrm{d}}{\mathrm{d} \bar{\chi}}\left(A^{(1)}-v_{\|}^{(1)}\right)+\left(A^{(1) \prime}-B_{\|}^{(1) \prime}-\frac{1}{2} h_{\|}^{(1) \prime}\right)\right]-\frac{2}{\mathcal{H}}\left[\left(2 A^{(1)}+h_{\|}^{(1)}\right)+\left(A^{(1)}-v_{\|}^{(1)}\right)\right] \\
& \times\left(A^{(1) \prime}-B_{\|}^{(1) \prime}-\frac{1}{2} h_{\|}^{(1) \prime}\right)+\frac{2}{\mathcal{H}}\left(2 A^{(1)}-B_{\|}^{(1)}\right)\left[\frac{\mathrm{d}}{\mathrm{d} \bar{\chi}}\left(2 A^{(1)}-B_{\|}^{(1)}\right)+\left(A^{(1) \prime}-B_{\|}^{(1) \prime}-\frac{1}{2} h_{\|}^{(1) \prime}\right)\right] \\
& +2\left\{\frac{1}{\mathcal{H}}\left(A^{(1)}-B_{\|}^{(1)}-\frac{1}{2} h_{\|}^{(1)}\right)+\frac{\mathcal{H}^{\prime}}{\mathcal{H}^{3}}\left(A^{(1)}-v_{\|}^{(1)}\right)+\frac{1}{\mathcal{H}^{2}}\left[\frac{\mathrm{d}}{\mathrm{d} \bar{\chi}}\left(A^{(1)}-v_{\|}^{(1)}\right)+\left(A^{(1) \prime}-B_{\|}^{(1) \prime}-\frac{1}{2} h_{\|}^{(1) \prime}\right)\right]\right\} \\
& \times\left[\frac{\mathrm{d}}{\mathrm{d} \bar{\chi}}\left(A^{(1)}-v_{\|}^{(1)}\right)+\left(A^{(1) \prime}-B_{\|}^{(1) \prime}-\frac{1}{2} h_{\|}^{(1) \prime}\right)\right]+\frac{2}{\mathcal{H}} v_{\perp i}^{(1)} \partial_{\perp}^{i}\left(A^{(1)}-B_{\|}^{(1)}-\frac{1}{2} h_{\|}^{(1)}\right)+\frac{2}{\mathcal{H}}\left(A^{(1)}-B_{\|}^{(1)}-\frac{1}{2} h_{\|}^{(1)}\right) \\
& \times\left[\frac{\mathrm{d}}{\mathrm{d} \bar{\chi}}\left(2 A^{(1)}-B_{\|}^{(1)}\right)-\partial_{\|}\left(A^{(1)}+v_{\|}^{(1)}-B_{\|}^{(1)}\right)\right]+\frac{2}{\mathcal{H}} \frac{\mathrm{d}}{\mathrm{d} \bar{\chi}} \partial_{\|}\left(A^{(1)}+v_{\|}^{(1)}-B_{\|}^{(1)}\right) T^{(1)}+2\left(B_{\perp}^{i(1)}+n^{j} h_{j k}^{(1)} \mathcal{P}^{i k}\right) \\
& \times\left[\frac{1}{2}\left(B_{i \perp}^{(1)}+n^{p} h_{p m}^{(1)} \mathcal{P}_{i}^{m}\right)-\frac{1}{\mathcal{H}} \partial_{\perp i}\left(A^{(1)}-v_{\|}^{(1)}\right)+\frac{1}{\mathcal{H}}\left(B_{\perp i}^{(1) \prime}+n^{j} h_{j k}^{(1) \prime} \mathcal{P}_{i}^{k}\right)\right]+4\left\{( - \frac { \mathcal { H } ^ { \prime } } { \mathcal { H } ^ { 3 } } + \frac { 1 } { \mathcal { H } } ) \left[\frac{\mathrm{d}}{\mathrm{d} \bar{\chi}}\left(A^{(1)}-v_{\|}^{(1)}\right)\right.\right. \\
& \left.\left.+\left(A^{(1) \prime}-B_{\|}^{(1) \prime}-\frac{1}{2} h_{\|}^{(1) \prime}\right)\right]-\frac{1}{\mathcal{H}^{2}} \frac{\mathrm{d}}{\mathrm{d} \bar{\chi}}\left[\frac{\mathrm{d}}{\mathrm{d} \bar{\chi}}\left(A^{(1)}-v_{\|}^{(1)}\right)+\left(A^{(1) \prime}-B_{\|}^{(1) \prime}-\frac{1}{2} h_{\|}^{(1) \prime}\right)\right]+\left(A^{(1)}-B_{\|}^{(1)}-\frac{1}{2} h_{\|}^{(1)}\right)\right\} I^{(1)} \\
& +2\left\{\frac{\mathrm{d}}{\mathrm{d} \bar{\chi}}\left(A^{(1)}-B_{\|}^{(1)}-\frac{1}{2} h_{\|}^{(1)}\right)+\frac{1}{\mathcal{H}} \frac{\mathrm{d}}{\mathrm{d} \bar{\chi}}\left[\frac{\mathrm{d}}{\mathrm{d} \bar{\chi}}\left(2 A^{(1)}-B_{\|}^{(1)}\right)+\left(A^{(1) \prime}-B_{\|}^{(1) \prime}-\frac{1}{2} h_{\|}^{(1) \prime}\right)\right]\right\} \int_{0}^{\bar{\chi}} \mathrm{d} \tilde{\chi}\left[2 A^{(1)}-B_{\|}^{(1)}\right. \\
& \left.+(\bar{\chi}-\tilde{\chi})\left(A^{(1) \prime}-B_{\|}^{(1) \prime}-\frac{1}{2} h_{\|}^{(1) \prime}\right)\right]+4\left[\frac{1}{\mathcal{H}} \partial_{\perp i}\left(A^{(1)}-v_{\|}^{(1)}\right)-\frac{1}{\mathcal{H}}\left(B_{\perp i}^{(1) \prime}+n^{j} h_{j k}^{(1) \prime} \mathcal{P}_{i}^{k}\right)+\frac{1}{\mathcal{H} \bar{\chi}} v_{\perp i}^{(1)}-\frac{1}{\mathcal{H}} \frac{\mathrm{d}}{\mathrm{d} \bar{\chi}} v_{\perp i}^{(1)}\right. \\
& \left.-2 S_{\perp}^{j(1)} \delta_{i j}\right] S_{\perp}^{i(1)}+\frac{2}{\mathcal{H}} \frac{\mathrm{d}}{\mathrm{d} \bar{\chi}}\left[\partial_{\perp i}\left(A^{(1)}+v_{\|}^{(1)}-B_{\|}^{(1)}\right)-\frac{1}{\bar{\chi}}\left(v_{\perp i}^{(1)}-B_{\perp i}^{(1)}\right)\right] \int_{0}^{\bar{\chi}} \mathrm{d} \tilde{\chi}\left\{\left(B_{\perp}^{i(1)}+n^{k} h_{k}^{j(1)} \mathcal{P}_{j}^{i}\right)\right. \\
& \left.+(\bar{\chi}-\tilde{\chi})\left[\tilde{\partial}_{\perp}^{i}\left(A^{(1)}-B_{\|}^{(1)}-\frac{1}{2} h_{\|}^{(1)}\right)+\frac{1}{\tilde{\chi}}\left(B_{\perp}^{i(1)}+n^{k} h_{k j}^{(1)} \mathcal{P}^{i j}\right)\right]\right\}-\frac{\mathcal{H}^{\prime}}{\mathcal{H}^{2}} \Delta \ln a^{(2)}+2\left\{-\frac{\mathcal{H}^{\prime}}{\mathcal{H}^{2}}\left(A^{(1)}-B_{\|}^{(1)}-\frac{1}{2} h_{\|}^{(1)}\right)\right. \\
& \left.-\frac{1}{\mathcal{H}} \frac{\mathrm{d}}{\mathrm{d} \bar{\chi}}\left(A^{(1)}-B_{\|}^{(1)}-\frac{1}{2} h_{\|}^{(1)}\right)-\left(\frac{\mathcal{H}^{\prime}}{\mathcal{H}^{3}}+\frac{1}{\mathcal{H}}\right)\left[\frac{\mathrm{d}}{\mathrm{d} \bar{\chi}}\left(A^{(1)}-v_{\|}^{(1)}\right)+\left(A^{(1) \prime}-B_{\|}^{(1) \prime}-\frac{1}{2} h_{\|}^{(1) \prime}\right)\right]\right\} \Delta \ln a^{(1)} \\
& +2 \int_{0}^{\bar{\chi}} \mathrm{d} \tilde{\chi}\left\{\left(A^{(1)}-B_{\|}^{(1)}-\frac{1}{2} h_{\|}^{(1)}\right)\left[2\left(A^{(1) \prime}-B_{\|}^{(1) \prime}-\frac{1}{2} h_{\|}^{(1) \prime}\right)+\frac{\mathrm{d}}{\mathrm{d} \tilde{\chi}}\left(2 A^{(1)}-B_{\|}^{(1)}\right)\right]-\left(B_{\perp}^{i(1)}+n^{k} h_{k}^{j(1)} \mathcal{P}_{j}^{i}\right)\right. \\
& \left.\times\left[\tilde{\partial}_{\perp i}\left(A^{(1)}-B_{\|}^{(1)}-\frac{1}{2} h_{\|}^{(1)}\right)+\frac{1}{\tilde{\chi}}\left(B_{\perp i}^{(1)}+n^{m} h_{m p}^{(1)} \mathcal{P}_{i}^{p}\right)\right]\right\}+\left[-\frac{\mathcal{H}^{\prime \prime}}{\mathcal{H}^{3}}+3\left(\frac{\mathcal{H}^{\prime}}{\mathcal{H}^{2}}\right)^{2}+\frac{\mathcal{H}^{\prime}}{\mathcal{H}^{2}}\right]\left(\Delta \ln a^{(1)}\right)^{2} \\
& +\partial_{\|} \Delta x_{\| \text {post-Born }}^{(2)}
\end{aligned}
$$


where

$$
\begin{aligned}
& \partial_{\|} \Delta x_{\| \text {post-Born }}^{(2)}=-2\left(A_{o}^{(1)}-B_{\| o}^{(1)}-\frac{1}{2} h_{\| o}^{(1)}\right)\left(3 v_{\| o}^{(1)}+2 h_{\| o}^{(1)}-\frac{1}{2} h_{j o}^{j(1)}\right)+2\left(A_{o}^{(1)}-v_{\| o}^{(1)}\right)\left\{-\left(A^{(1)}-B_{\|}^{(1)}-\frac{1}{2} h_{\|}^{(1)}\right)\right. \\
& \left.+\frac{\bar{\chi}}{\mathcal{H}} \frac{\mathrm{d}}{\mathrm{d} \bar{\chi}}\left[\frac{\mathrm{d}}{\mathrm{d} \bar{\chi}}\left(2 A^{(1)}-B_{\|}^{(1)}\right)+\left(A^{(1) \prime}-B_{\|}^{(1) \prime}-\frac{1}{2} h_{\|}^{(1) \prime}\right)\right]\right\}+2 \bar{\chi}\left(A_{o}^{(1)}-v_{\| o}^{(1)}\right) \frac{\mathrm{d}}{\mathrm{d} \bar{\chi}}\left(A^{(1)}-B_{\|}^{(1)}-\frac{1}{2} h_{\|}^{(1)}\right) \\
& +2\left(B_{\perp o}^{i(1)}-v_{\perp o}^{i(1)}+\frac{1}{2} n^{k} h_{k o}^{j(1)} \mathcal{P}_{j}^{i}\right)\left\{\bar{\chi} \partial_{\perp i}\left(A^{(1)}-B_{\|}^{(1)}-\frac{1}{2} h_{\|}^{(1)}\right)+\left(B_{\perp i}^{(1)}+n^{j} h_{j}^{k(1)} \mathcal{P}_{i k}\right)-\frac{1}{\mathcal{H}} \partial_{\perp i}\left(2 A^{(1)}-B_{\|}^{(1)}\right)\right. \\
& +\frac{\bar{\chi}}{\mathcal{H}} \partial_{\perp i} \frac{\mathrm{d}}{\mathrm{d} \bar{\chi}}\left(2 A^{(1)}-B_{\|}^{(1)}\right)+\frac{\bar{\chi}}{\mathcal{H}} \partial_{\perp i}\left(A^{(1) \prime}-B_{\|}^{(1) \prime}-\frac{1}{2} h_{\|}^{(1) \prime}\right)-\frac{1}{\bar{\chi} \mathcal{H}} B_{\perp i}^{(1)}+\frac{1}{\mathcal{H}} \frac{\mathrm{d}}{\mathrm{d} \bar{\chi}} B_{\perp i}^{(1)} \\
& \left.+\frac{1}{\mathcal{H}}\left(B_{\perp i}^{(1) \prime}+n^{j} h_{j k}^{(1) \prime} \mathcal{P}_{i}^{k}\right)+2 S_{\perp}^{j(1)}\right\}-4\left(-B_{\| o}^{(1)}+v_{\| o}^{(1)}-\frac{3}{4} h_{\| o}^{(1)}+\frac{1}{4} h_{m o}^{m(1)}\right) \int_{0}^{\bar{\chi}} \frac{\mathrm{d} \tilde{\chi}}{\tilde{\chi}}\left(A^{(1)}-B_{\|}^{(1)}-\frac{1}{2} h_{\|}^{(1)}\right) \\
& -2\left\{\left(A^{(1) \prime}-B_{\|}^{(1) \prime}-\frac{1}{2} h_{\|}^{(1) \prime}\right)+\frac{1}{\mathcal{H}}\left[\frac{\mathrm{d}}{\mathrm{d} \bar{\chi}}\left(2 A^{(1) \prime}-B_{\|}^{(1) \prime}\right)+\left(A^{(1) \prime \prime}-B_{\|}^{(1) \prime \prime}-\frac{1}{2} h_{\|}^{(1) \prime \prime}\right)\right]\right\} T^{(1)} \\
& -2\left\{\frac{\mathrm{d}}{\mathrm{d} \bar{\chi}}\left(A^{(1)}-B_{\|}^{(1)}-\frac{1}{2} h_{\|}^{(1)}\right)+\frac{1}{\mathcal{H}} \frac{\mathrm{d}}{\mathrm{d} \bar{\chi}}\left[\frac{\mathrm{d}}{\mathrm{d} \bar{\chi}}\left(2 A^{(1)}-B_{\|}^{(1)}\right)+\left(A^{(1) \prime}-B_{\|}^{(1) \prime}-\frac{1}{2} h_{\|}^{(1) \prime}\right)\right]\right\} \\
& \times \int_{0}^{\bar{\chi}} \mathrm{d} \tilde{\chi}\left(A^{(1)}+\frac{1}{2} h_{\|}^{(1)}-2 I^{(1)}\right)+2\left(A^{(1)}-B_{\|}^{(1)}-\frac{1}{2} h_{\|}^{(1)}\right)\left(A^{(1)}+\frac{1}{2} h_{\|}^{(1)}-2 I^{(1)}\right) \\
& -4\left(A^{(1)}-B_{\|}^{(1)}-\frac{1}{2} h_{\|}^{(1)}\right) \kappa^{(1)}-2\left\{\partial_{\perp i}\left(A^{(1)}-B_{\|}^{(1)}-\frac{1}{2} h_{\|}^{(1)}\right)+\frac{1}{\bar{\chi}}\left(B_{\perp i}^{(1)}+n^{j} h_{j}^{k(1)} \mathcal{P}_{i k}\right)\right. \\
& +\frac{1}{\mathcal{H}} \partial_{\perp i} \frac{\mathrm{d}}{\mathrm{d} \bar{\chi}}\left(2 A^{(1)}-B_{\|}^{(1)}\right)+\frac{1}{\mathcal{H}} \partial_{\perp i}\left(A^{(1) \prime}-B_{\|}^{(1) \prime}-\frac{1}{2} h_{\|}^{(1) \prime}\right)-\frac{1}{\bar{\chi} \mathcal{H}}\left[\partial_{\perp i}\left(2 A^{(1)}-B_{\|}^{(1)}\right)\right. \\
& \left.\left.+\left(\frac{1}{\bar{\chi}} B_{\perp i}^{(1)}-\frac{\mathrm{d}}{\mathrm{d} \bar{\chi}} B_{\perp i}^{(1)}\right)-\left(B_{\perp i}^{(1) \prime}+n^{j} h_{j k}^{(1) \prime} \mathcal{P}_{i}^{k}\right)\right]\right\} \int_{0}^{\bar{\chi}} \mathrm{d} \tilde{\chi}\left(B_{\perp}^{i(1)}+n^{k} h_{k}^{j(1)} \mathcal{P}_{j}^{i}-2 S_{\perp}^{i(1)}\right) \\
& +2 \int_{0}^{\bar{\chi}} \mathrm{d} \tilde{\chi}\left\{-\left(A^{(1)}-B_{\|}^{(1)}-\frac{1}{2} h_{\|}^{(1)}\right)\left[\frac{\mathrm{d}}{\mathrm{d} \tilde{\chi}}\left(A^{(1)}+\frac{1}{2} h_{\|}^{(1)}\right)+2\left(A^{(1) \prime}-B_{\|}^{(1) \prime}-\frac{1}{2} h_{\|}^{(1) \prime}\right)\right]\right. \\
& -\left[\tilde{\partial}_{\perp j}\left(A^{(1)}-B_{\|}^{(1)}-\frac{1}{2} h_{\|}^{(1)}\right)+\frac{1}{\bar{\chi}}\left(B_{\perp j}^{(1)}+n^{m} h_{m}^{k(1)} \mathcal{P}_{j k}\right)\right]\left[-\left(B_{\perp}^{j(1)}+n^{l} h_{l}^{p(1)} \mathcal{P}_{p}^{j}\right)+2 S_{\perp}^{j(1)}\right] \\
& +\left(A^{(1)}-B_{\|}^{(1)}-\frac{1}{2} h_{\|}^{(1)}\right)\left[\tilde{\partial}_{\perp m}\left(B^{m(1)}+n^{l} h_{l}^{m(1)}\right)-\frac{2}{\tilde{\chi}}\left(B_{\|}^{(1)}+h_{\|}^{(1)}\right)-2 \tilde{\partial}_{\perp m} S_{\perp}^{m(1)}\right] \\
& \left.+2\left[\frac{\mathrm{d}}{\mathrm{d} \bar{\chi}}\left(A^{(1)}-B_{\|}^{(1)}-\frac{1}{2} h_{\|}^{(1)}\right)-\frac{1}{\tilde{\chi}}\left(A^{(1)}-B_{\|}^{(1)}-\frac{1}{2} h_{\|}^{(1)}\right)\right] \kappa^{(1)}\right\} .
\end{aligned}
$$

From Eq. 131) and using Eqs. 125 (126) and (127) we obtain the coordinate convergence lensing term at second order

$$
\kappa^{(2)}=-\frac{1}{2} \partial_{\perp i} \Delta x_{\perp}^{i(2)}=\kappa_{1}^{(2)}+\kappa_{2}^{(2)}+\kappa_{3}^{(2)}+\kappa_{4}^{(2)}+\kappa_{\text {post-Born }}^{(2)}
$$

where

$$
\begin{aligned}
& \kappa_{1}^{(2)}=\frac{1}{2} \int_{0}^{\bar{\chi}} \mathrm{d} \tilde{\chi}(\bar{\chi}-\tilde{\chi}) \frac{\tilde{\chi}}{\bar{\chi}} \tilde{\nabla}_{\perp}^{2}\left(A^{(2)}-B_{\|}^{(2)}-\frac{1}{2} h_{\|}^{(2)}\right), \\
& \kappa_{2}^{(2)}=\frac{1}{2} \int_{0}^{\bar{\chi}} \mathrm{d} \tilde{\chi}\left[\tilde{\partial}_{\perp}^{i} B_{i}^{(2)}-\frac{2}{\tilde{\chi}} B_{\|}^{(2)}+\mathcal{P}^{i j} n^{k} \tilde{\partial}_{i} h_{j k}^{(2)}+\frac{1}{\tilde{\chi}}\left(h_{i}^{i(2)}-3 h_{\|}^{(2)}\right)\right],
\end{aligned}
$$




$$
\begin{aligned}
& \kappa_{3}^{(2)}=\left\{-\frac{2}{\bar{\chi}} B_{\|}^{(1)}+\partial_{\perp}^{i} B_{i}^{(1)}+\frac{1}{\bar{\chi}}\left(h_{i}^{i(1)}-3 h_{\|}^{(1)}\right)+\mathcal{P}^{i j} n^{k} \partial_{i} h_{j k}^{(1)}+\frac{1}{\bar{\chi}} \int_{0}^{\bar{\chi}} \mathrm{d} \tilde{\chi}\left[\tilde{\partial}_{\perp}^{i} B_{i}^{(1)}+\mathcal{P}^{i j} n^{k} \tilde{\partial}_{i} h_{j k}^{(1)}\right.\right. \\
& \left.\left.-\frac{1}{\tilde{\chi}}\left(2 B_{\|}^{(1)}+3 h_{\|}^{(1)}-h_{i}^{i(1)}\right)\right]+\int_{0}^{\bar{\chi}} \mathrm{d} \tilde{\chi} \frac{\tilde{\chi}}{\bar{\chi}} \tilde{\nabla}_{\perp}^{2}\left(A^{(1)}-B_{\|}^{(1)}-\frac{1}{2} h_{\|}^{(1)}\right)\right\} \times\left\{\int _ { 0 } ^ { \overline { \chi } } \mathrm { d } \tilde { \chi } \left[2 A^{(1)}-B_{\|}^{(1)}\right.\right. \\
& \left.\left.+(\bar{\chi}-\tilde{\chi})\left(A^{(1) \prime}-B_{\|}^{(1) \prime}-\frac{1}{2} h_{\|}^{(1) \prime}\right)\right]-\frac{1}{\mathcal{H}} \Delta \ln a^{(1)}\right\}+\left(B_{\perp}^{i(1)}+n^{k} h_{k}^{j(1)} \mathcal{P}_{j}^{i}-2 S_{\perp}^{i(1)}\right) \\
& \times\left\{\int_{0}^{\bar{\chi}} \mathrm{d} \tilde{\chi} \frac{\tilde{\chi}}{\bar{\chi}}\left[\tilde{\partial}_{\perp i}\left(2 A^{(1)}-B_{\|}^{(1)}\right)+(\bar{\chi}-\tilde{\chi}) \tilde{\partial}_{\perp i}\left(A^{(1) \prime}-B_{\|}^{(1) \prime}-\frac{1}{2} h_{\|}^{(1) \prime}\right)\right]-\frac{1}{\mathcal{H}} \partial_{\perp i} \Delta \ln a^{(1)}\right\} \\
& +\int_{0}^{\bar{\chi}} \mathrm{d} \tilde{\chi}\left[\frac{4}{\tilde{\chi}} A^{(1)} B_{\|}^{(1)}-4 A^{(1)} \tilde{\partial}_{\perp}^{i} B_{i}^{(1)}-\frac{2}{\tilde{\chi}}\left(B_{\|}^{(1)}\right)^{2}+B_{\|}^{(1)} \tilde{\partial}_{\perp}^{i} B_{i}^{(1)}-\frac{3}{\tilde{\chi}} A^{(1)} h_{i}^{i(1)}+\frac{9}{\tilde{\chi}} A^{(1)} h_{\|}^{(1)}\right. \\
& -3 A^{(1)} \mathcal{P}^{i j} n^{k} \tilde{\partial}_{i} h_{j k}^{(1)}+\frac{2}{\tilde{\chi}} B_{\|}^{(1)} h_{\|}^{(1)}-h_{\|}^{(1)} \tilde{\partial}_{\perp}^{i} B_{i}^{(1)}-\frac{3}{2 \tilde{\chi}} h_{\|}^{(1)} h_{i}^{i(1)}+\frac{9}{2 \tilde{\chi}}\left(h_{\|}^{(1)}\right)^{2}-\frac{3}{2} h_{\|}^{(1)} \mathcal{P}^{i j} n^{k} \tilde{\partial}_{i} h_{j k}^{(1)} \\
& \left.-4\left(\frac{2}{\tilde{\chi}} B_{\|}^{(1)}-\tilde{\partial}_{\perp}^{i} B_{i}^{(1)}-\frac{1}{\tilde{\chi}} h_{i}^{i(1)}+\frac{3}{\tilde{\chi}} h_{\|}^{(1)}-\mathcal{P}^{i j} n^{k} \tilde{\partial}_{i} h_{j k}^{(1)}\right) I^{(1)}\right]+\int_{0}^{\bar{\chi}} \mathrm{d} \tilde{\chi} \frac{\tilde{\chi}}{\bar{\chi}}\left[-\frac{4}{\tilde{\chi}} A^{(1)} B_{\|}^{(1)}+2 A^{(1)} \tilde{\partial}_{\perp}^{i} B_{i}^{(1)}\right. \\
& +\frac{2}{\tilde{\chi}} A^{(1)} h_{i}^{i(1)}-\frac{6}{\tilde{\chi}} A^{(1)} h_{\|}^{(1)}+2 A^{(1)} \mathcal{P}^{i j} n^{k} \tilde{\partial}_{i} h_{j k}^{(1)}+\frac{1}{\tilde{\chi}} B_{\|}^{(1)} h_{i}^{i(1)}-\frac{3}{\tilde{\chi}} B_{\|}^{(1)} h_{\|}^{(1)}-h_{\|}^{(1)} \tilde{\partial}_{\perp}^{i} B_{i}^{(1)}-\frac{4}{\tilde{\chi}}\left(h_{\|}^{(1)}\right)^{2} \\
& +h_{\|}^{(1)} \mathcal{P}^{i j} n^{k} \tilde{\partial}_{i} h_{j k}^{(1)}-2 \tilde{\partial}_{\perp i} A^{(1)} B_{\perp}^{i(1)}+\frac{1}{\tilde{\chi}} B_{\perp i}^{(1)} B_{\perp}^{i(1)}+n^{j} B_{\perp}^{i(1)} \tilde{\partial}_{\perp i} B_{j}^{(1)}-\tilde{\partial}_{i} A^{(1)} n^{j} h_{j k}^{(1)} \mathcal{P}^{k i}+\frac{2}{\tilde{\chi}} n^{p} h_{p q}^{(1)} \mathcal{P}^{q j} h_{j k}^{(1)} n^{k} \\
& -\frac{1}{2} n^{j} h_{j k}^{(1)} \mathcal{P}^{k i} n^{p} n^{q} \tilde{\partial}_{\perp i} h_{p q}^{(1)}-h_{l}^{k(1)} \mathcal{P}_{q}^{l} \tilde{\partial}_{\perp k} B^{q(1)}-\frac{1}{\tilde{\chi}} \mathcal{P}_{k}^{m} \mathcal{P}_{p}^{l} h_{m}^{p(1)} h_{l}^{k(1)}+\frac{3}{\tilde{\chi}} n^{l} B_{\perp k}^{(1)} h_{l}^{k(1)}-B_{\perp}^{l(1)} \tilde{\partial}_{\perp k} h_{l}^{k(1)} \\
& +\frac{2}{\tilde{\chi}} h_{\|}^{(1)} h_{i}^{i(1)}-n^{m} h_{m}^{p(1)} \mathcal{P}_{p}^{l} \tilde{\partial}_{\perp k} h_{l}^{k(1)}+4\left(B_{\perp}^{i(1)}+n^{j} h_{j k}^{(1)} \mathcal{P}^{i k}\right) \tilde{\partial}_{\perp i} I^{(1)}+2\left(-\frac{3}{\tilde{\chi}} n^{l} \mathcal{P}_{k j} h_{l}^{k(1)}+\mathcal{P}_{j}^{l} \tilde{\partial}_{\perp k} h_{l}^{k(1)}\right) S_{\perp}^{j(1)} \\
& \left.-\frac{2}{\tilde{\chi}} \mathcal{P}_{k}^{l} h_{l}^{k(1)} S_{\|}^{(1)}+2 \mathcal{P}_{m}^{l} h_{l}^{k(1)} \tilde{\partial}_{\perp k} S^{m(1)}\right]+\int_{0}^{\bar{\chi}} \mathrm{d} \tilde{\chi}(\bar{\chi}-\tilde{\chi}) \frac{\tilde{\chi}}{\bar{\chi}}\left\{\tilde{\partial}_{\perp i}\left(A^{(1) \prime}-B_{\|}^{(1) \prime}-\frac{1}{2} h_{\|}^{(1) \prime}\right)\right. \\
& \times\left(B_{\perp}^{i(1)}+n^{j} h_{j k}^{(1)} \mathcal{P}^{i k}\right)+\left(A^{(1) \prime}-B_{\|}^{(1) \prime}-\frac{1}{2} h_{\|}^{(1) \prime}\right)\left(-\frac{2}{\tilde{\chi}} B_{\|}^{(1)}+\tilde{\partial}_{\perp}^{i} B_{i}^{(1)}+\frac{1}{\tilde{\chi}} h_{i}^{i(1)}-\frac{3}{\tilde{\chi}} h_{\|}^{(1)}+n^{k} \tilde{\partial}_{\perp}^{j(1)} h_{j k}^{(1)}\right) \\
& -\left(2 \partial_{\perp i} A^{(1)}-\frac{1}{\tilde{\chi}} B_{\perp i}^{(1)}-n_{k} \tilde{\partial}_{\perp i} B_{k}^{(1)}\right) \frac{\mathrm{d}}{\mathrm{d} \tilde{\chi}}\left(B_{\perp}^{i(1)}+n^{j} h_{j k}^{(1)} \mathcal{P}^{i k}\right)-\left(2 A^{(1)}-B_{\|}^{(1)}\right) \frac{\mathrm{d}}{\mathrm{d} \tilde{\chi}}\left(-\frac{2}{\tilde{\chi}} B_{\|}^{(1)}+\tilde{\partial}_{\perp}^{i} B_{i}^{(1)}\right. \\
& \left.+\frac{1}{\tilde{\chi}} h_{i}^{i(1)}-\frac{3}{\tilde{\chi}} h_{\|}^{(1)}+n^{k} \tilde{\partial}_{\perp}^{j(1)} h_{j k}^{(1)}\right)-2\left(-\mathcal{P}_{j q} \tilde{\partial}_{\perp i} B^{q(1)}-n^{k} \mathcal{P}_{j q} \tilde{\partial}_{\perp i} h_{k}^{q(1)}+2 \mathcal{P}_{j m} \tilde{\partial}_{\perp j} S^{m(1)}\right) \\
& \times\left(\mathcal{P}^{l[j} \partial_{\perp}^{i]} B_{l}^{(1)}+n^{m} \mathcal{P}^{l[j} \partial_{\perp}^{i]} h_{l m}^{(1)}\right)+\left(B_{\perp j}^{(1)}+n^{p} h_{p}^{k(1)} \mathcal{P}_{j k}-2 \delta_{j p} S_{\perp}^{p(1)}\right)\left(\mathcal{P}^{j l} \tilde{\nabla}_{\perp}^{2} B_{l}^{(1)}-\tilde{\partial}_{\perp}^{j} \tilde{\partial}_{\perp}^{k} B_{k}^{(1)}\right. \\
& \left.+\mathcal{P}^{j l} n^{m} \tilde{\nabla}_{\perp}^{2} h_{l m}^{(1)}-n^{m} \tilde{\partial}_{\perp}^{j} \tilde{\partial}_{\perp}^{k} h_{k m}^{(1)}+\frac{1}{\tilde{\chi}} n^{m} n^{k} \tilde{\partial}_{\perp}^{j} h_{k m}^{(1)}+\frac{1}{\tilde{\chi}} \mathcal{P}^{j l} \tilde{\partial}_{\perp}^{m} h_{l m}^{(1)}-\frac{1}{\tilde{\chi}} \tilde{\partial}_{\perp}^{j} h_{i}^{i(1)}\right) \\
& -\tilde{\partial}_{\perp i}\left(A^{(1)}-B_{\|}^{(1)}-\frac{1}{2} h_{\|}^{(1)}\right)\left[\tilde{\partial}_{\perp}^{i}\left(B_{\|}^{(1)}+h_{\|}^{(1)}\right)-\partial_{\|}\left(B_{\perp}^{i(1)}+n^{p} h_{p q}^{(1)} \mathcal{P}^{i q}\right)-\frac{1}{\tilde{\chi}}\left(B_{\perp}^{i(1)}+2 n^{p} h_{p q}^{(1)} \mathcal{P}^{i q}\right)\right] \\
& -\left(A^{(1)}-B_{\|}^{(1)}-\frac{1}{2} h_{\|}^{(1)}\right)\left[\tilde{\nabla}_{\perp}^{2}\left(B_{\|}^{(1)}+h_{\|}^{(1)}\right)-\tilde{\partial}_{\|}\left(-\frac{2}{\tilde{\chi}} B_{\|}^{(1)}+\tilde{\partial}_{\perp}^{i} B_{i}^{(1)}+\frac{1}{\tilde{\chi}} h_{i}^{i(1)}-\frac{3}{\tilde{\chi}} h_{\|}^{(1)}+n^{k} \tilde{\partial}_{\perp}^{j(1)} h_{j k}^{(1)}\right)\right] \\
& -2\left(2 \tilde{\partial}_{\perp}^{i} A^{(1)}-\frac{1}{\tilde{\chi}} B_{\perp j}^{(1)}-n_{k} \tilde{\partial}_{\perp i} B_{k}^{(1)}-2 \tilde{\partial}_{\perp}^{i} I^{(1)}\right)\left[\tilde{\partial}_{\perp}^{i}\left(A^{(1)}-B_{\|}^{(1)}-\frac{1}{2} h_{\|}^{(1)}\right)+\frac{1}{\tilde{\chi}}\left(B_{\perp}^{i(1)}+n^{j} h_{j k}^{(1)} \mathcal{P}^{k i}\right)\right] \\
& \left.-2\left(2 A^{(1)}-B_{\|}^{(1)}-2 I^{(1)}\right) \tilde{\nabla}_{\perp}^{2}\left(A^{(1)}-B_{\|}^{(1)}-\frac{1}{2} h_{\|}^{(1)}\right)\right\},
\end{aligned}
$$




$$
\begin{aligned}
& \kappa_{4}^{(2)}=B_{\| o}^{(2)}-v_{\| o}^{(2)}+\frac{3}{4} h_{\| o}^{(2)}-\frac{1}{4} h_{i o}^{i(2)}-2 A_{o}^{(1)} B_{\| o}^{(1)}+2 A_{o}^{(1)} v_{\| o}^{(1)}-\frac{1}{2} A_{o}^{(1)} h_{i o}^{i(1)}+\frac{3}{2} A_{o}^{(1)} h_{\| o}^{(1)}+\frac{1}{2} v_{\perp i o}^{(1)} v_{\perp o}^{i(1)}-\left(v_{\| o}^{(1)}\right)^{2} \\
& +v_{\perp i o}^{(1)} B_{\perp o}^{i(1)}-2 v_{\| o}^{(1)} B_{\| o}^{(1)}-\frac{1}{2} B_{\perp i o}^{(1)} B_{\perp o}^{i(1)}+\left(B_{\| o}^{(1)}\right)^{2}-\frac{7}{2} v_{\| o}^{(1)} h_{\| o}^{(1)}+\frac{1}{2} v_{\| o}^{(1)} h_{i o}^{i(1)}-\frac{3}{2} v_{i o}^{(1)} n^{k} h_{k o}^{j(1)} \mathcal{P}_{j}^{i} \\
& +\frac{1}{8} h_{k o}^{j(1)} h_{j o}^{k(1)}-\frac{3}{8} n^{i} h_{i o}^{j(1)} h_{j o}^{k(1)} n_{k}+v_{\perp i o}^{(1)}\left\{\left(B_{\perp}^{i(1)}+n^{k} h_{k j}^{(1)} \mathcal{P}^{i j}\right)-2 S_{\perp}^{i(1)}-\int_{0}^{\bar{\chi}} \mathrm{d} \tilde{\chi}\left[\frac{2}{\tilde{\chi}}\left(B_{\perp}^{i(1)}+n^{k} h_{k}^{j(1)} \mathcal{P}_{j}^{i}\right)\right.\right. \\
& \left.\left.+2\left(1-\frac{\tilde{\chi}}{\bar{\chi}}\right) \tilde{\partial}_{\perp}^{i}\left(A^{(1)}-B_{\|}^{(1)}-\frac{1}{2} h_{\|}^{(1)}\right)\right]\right\}+\left(A_{o}^{(1)}-v_{\| o}^{(1)}\right)\left\{2 B_{\|}^{(1)}-\bar{\chi} \partial_{\perp i} B^{i(1)}+3 h_{\|}^{(1)}-h_{i}^{i(1)}\right. \\
& -\bar{\chi} n^{k} \partial_{\perp j} h_{k}^{j(1)}-\int_{0}^{\bar{\chi}} \mathrm{d} \tilde{\chi}\left[\tilde{\partial}_{\perp i} B^{i(1)}+n^{k} \tilde{\partial}_{\perp j} h_{k}^{j(1)}-\frac{2}{\tilde{\chi}} B_{\|}^{(1)}+\frac{1}{\tilde{\chi}}\left(h_{i}^{i(1)}-3 h_{\|}^{(1)}\right)+\tilde{\chi} \tilde{\nabla}_{\perp}^{2}\left(A^{(1)}-B_{\|}^{(1)}-\frac{1}{2} h_{\|}^{(1)}\right)\right] \\
& \left.+4 \kappa_{2}^{(1)}+4 \kappa_{1}^{(1)}\right\}-\frac{1}{\bar{\chi}}\left(B_{\| o}^{(1)}-v_{\| o}^{(1)}+\frac{1}{2} h_{\| o}^{(1)}\right) \int_{0}^{\bar{\chi}} \mathrm{d} \tilde{\chi}\left(h_{i}^{i(1)}-h_{\|}^{(1)}\right)+\frac{1}{2 \bar{\chi}} \mathcal{P}_{i}^{k} h_{k o}^{l(1)} \mathcal{P}_{j l} \int_{0}^{\bar{\chi}} \mathrm{d} \tilde{\chi}\left(\mathcal{P}^{j m} h_{m}^{p(1)} \mathcal{P}_{p}^{i(1)}\right) \\
& +\left(B_{\perp j o}^{(1)}-v_{\perp j o}^{(1)}+\frac{1}{2} n^{k} h_{k o}^{l(1)} \mathcal{P}_{j l}\right) \int_{0}^{\bar{\chi}} \mathrm{d} \tilde{\chi}\left\{-\mathcal{P}^{j l} \tilde{\partial}_{\perp}^{m} h_{l m}^{(1)}+\frac{\tilde{\chi}}{\bar{\chi}}\left[-\frac{3}{\tilde{\chi}} n_{p} h_{m}^{p(1)} \mathcal{P}^{m j}+2 \mathcal{P}^{j m} \partial_{\perp p} h_{m}^{p(1)}\right.\right. \\
& -(\bar{\chi}-\tilde{\chi})\left(\mathcal{P}^{j l} \tilde{\nabla}_{\perp}^{2} B_{l}^{(1)}-\tilde{\partial}_{\perp}^{j} \tilde{\partial}_{\perp}^{k} B_{k}^{(1)}+\mathcal{P}^{j l} n^{m} \tilde{\nabla}_{\perp}^{2} h_{l m}^{(1)}-n^{m} \tilde{\partial}_{\perp}^{j} \tilde{\partial}_{\perp}^{k} h_{k m}^{(1)}\right) \\
& \left.\left.-\left(\frac{\bar{\chi}}{\tilde{\chi}}-1\right)\left(n^{m} n^{k} \tilde{\partial}_{\perp}^{j} h_{k m}^{(1)}-\tilde{\partial}_{\perp}^{j} h_{k}^{k(1)}\right)\right]\right\}+\left[\frac{2}{\bar{\chi}}\left(B_{\| o}^{(1)}-v_{\| o}^{(1)}\right)-\frac{1}{2 \bar{\chi}} h_{i o}^{i(1)}+\frac{3}{2 \bar{\chi}} h_{\| o}^{(1)}\right] \\
& \times\left\{\int_{0}^{\bar{\chi}} \mathrm{d} \tilde{\chi}\left[2 A^{(1)}-B_{\|}^{(1)}+(\bar{\chi}-\tilde{\chi})\left(A^{(1) \prime}-B_{\|}^{(1) \prime}-\frac{1}{2} h_{\|}^{(1) \prime}\right)\right]-\frac{1}{\mathcal{H}} \Delta \ln a^{(1)}\right\} \\
& -\left(B_{\perp o}^{i(1)}-v_{\perp o}^{i(1)}+\frac{1}{2} n^{k} h_{k o}^{j(1)} \mathcal{P}_{j}^{i}\right)\left\{\int_{0}^{\bar{\chi}} \mathrm{d} \tilde{\chi} \frac{\tilde{\chi}}{\bar{\chi}}\left[\tilde{\partial}_{\perp i}\left(2 A^{(1)}-B_{\|}^{(1)}\right)+(\bar{\chi}-\tilde{\chi}) \tilde{\partial}_{\perp i}\left(A^{(1) \prime}-B_{\|}^{(1) \prime}-\frac{1}{2} h_{\|}^{(1) \prime}\right)\right]\right. \\
& \left.-\frac{1}{\mathcal{H}} \partial_{\perp i} \Delta \ln a^{(1)}\right\} \text {. }
\end{aligned}
$$

Here

$$
\kappa_{\text {post-Born }}^{(2)}=\kappa_{1 \text { post-Born }}^{(2)}+\kappa_{2 \text { post-Born }}^{(2)}+\kappa_{3 \text { post-Born }}^{(2)},
$$

where

$$
\begin{aligned}
& \kappa_{1 \text { post }- \text { Born }}^{(2)}=-\left(B_{\perp}^{(1) i}+n^{j} h_{j}^{k(1)} \mathcal{P}_{k}^{i}\right) \int_{0}^{\bar{\chi}} \mathrm{d} \tilde{\chi} \frac{\tilde{\chi}}{\bar{\chi}}\left[\tilde{\partial}_{\perp i}\left(A^{(1)}+\frac{1}{2} h_{\|}^{(1)}\right)-2 \tilde{\partial}_{\perp i} I^{(1)}\right]-\left[\partial_{\perp i}\left(B^{i(1)}+n^{k} h_{k}^{i(1)}\right)\right. \\
& \left.-\frac{2}{\tilde{\chi}}\left(B_{\|}^{(1)}+h_{\|}^{(1)}\right)\right] \int_{0}^{\bar{\chi}} \mathrm{d} \tilde{\chi}\left(A^{(1)}+\frac{1}{2} h_{\|}^{(1)}-2 I^{(1)}\right)+\int_{0}^{\bar{\chi}} \mathrm{d} \tilde{\chi} \frac{\tilde{\chi}}{\bar{\chi}}\left\{2\left[\tilde{\partial}_{\perp m}\left(B^{m(1)}+n^{l} h_{l}^{m(1)}\right)-\frac{2}{\tilde{\chi}}\left(B_{\|}^{(1)}+h_{\|}^{(1)}\right)\right]\right. \\
& \times\left(A^{(1)}+\frac{1}{2} h_{\|}^{(1)}-2 I^{(1)}\right)+2\left(B_{\perp}^{(1) i}+n^{j} h_{j}^{k(1)} \mathcal{P}_{k}^{i}\right)\left[\tilde{\partial}_{\perp i}\left(A^{(1)}+\frac{1}{2} h_{\|}^{(1)}\right)-2 \tilde{\partial}_{\perp i} I^{(1)}\right] \\
& +\left[\tilde{\nabla}_{\perp}^{2}\left(A^{(1)}-B_{\|}^{(1)}-\frac{1}{2} h_{\|}^{(1)}\right)+\frac{1}{\tilde{\chi}} \tilde{\partial}_{\perp m}\left(B^{m(1)}+n^{l} h_{l}^{m(1)}\right)-\frac{2}{\tilde{\chi}^{2}}\left(B_{\|}^{(1)}+h_{\|}^{(1)}\right)\right] \delta x_{\|}^{(1)} \\
& +\left[\partial_{\perp i}\left(A^{(1)}-B_{\|}^{(1)}-\frac{1}{2} h_{\|}^{(1)}\right)+\frac{1}{\bar{\chi}}\left(B_{\perp i}^{(1)}+n^{k} h_{k}^{j(1)} \mathcal{P}_{i j}\right)\right] \partial_{\perp}^{i} \delta x_{\|}^{(1)}-\left[\tilde{\partial}_{\perp m}\left(B^{m(1) \prime}+n^{l} h_{l}^{m(1) \prime}\right)\right. \\
& \left.-\frac{2}{\tilde{\chi}}\left(B_{\|}^{(1) \prime}+h_{\|}^{(1) \prime}\right)\right] T^{(1)}-\left(B_{\perp i}^{(1) \prime}+n^{j} h_{j k}^{(1) \prime} \mathcal{P}_{i}^{k}\right) \tilde{\partial}_{\perp}^{i} T^{(1)}+\left[-\frac{1}{\tilde{\chi}} \tilde{\partial}_{\perp i}\left(B_{\|}^{(1)}+h_{\|}^{(1)}\right)+\frac{1}{\tilde{\chi}^{2}}\left(B_{\perp i}^{(1)}+n^{k} h_{k}^{j(1)} \mathcal{P}_{i j}\right)\right. \\
& \left.+\frac{2}{\tilde{\chi}^{2}} n^{k} h_{k i}^{(1)}+\tilde{\partial}_{\perp i} \tilde{\partial}_{\perp m}\left(B^{m(1)}+n^{k} h_{k}^{m(1)}\right)-\frac{1}{\tilde{\chi}} \tilde{\partial}_{\perp m} h_{i}^{m(1)}+\frac{1}{\tilde{\chi}} \tilde{\partial}_{\perp i}\left(A^{(1)}-B_{\|}^{(1)}-\frac{1}{2} h_{\|}^{(1)}\right)\right] \delta x_{\perp}^{i(1)} \\
& -\frac{2}{\tilde{\chi}}\left(A^{(1)}-B_{\|}^{(1)}-\frac{1}{2} h_{\|}^{(1)}\right) \kappa^{(1)}+\frac{4}{\tilde{\chi}}\left(B_{\|}^{(1)}+h_{\|}^{(1)}\right) \kappa^{(1)}-2 \tilde{\partial}_{\perp m}\left(B^{m(1)}+n^{k} h_{k}^{m(1)}\right) \kappa^{(1)} \\
& \left.-2\left(B^{m(1)}+n^{l} h_{l}^{m(1)}\right) \tilde{\partial}_{\perp m} \kappa^{(1)}-\left[\partial_{\perp}^{j}\left(B^{m(1)}+n^{k} h_{k}^{m(1)}\right)-\frac{1}{\tilde{\chi}} h^{j m(1)}\right]\left(\gamma_{m j}^{(1)}+\mathcal{P}_{j m} \kappa^{(1)}+\theta_{j m}^{(1)}\right)\right\}
\end{aligned}
$$




$$
\begin{aligned}
& \kappa_{2 \text { post-Born }}^{(2)}=\int_{0}^{\bar{\chi}} \mathrm{d} \tilde{\chi}(\bar{\chi}-\tilde{\chi}) \frac{\tilde{\chi}}{\bar{\chi}}\left\{-\left[\tilde{\nabla}_{\perp}^{2}\left(A^{(1) \prime}-B_{\|}^{(1) \prime}-\frac{1}{2} h_{\|}^{(1) \prime}\right)+\frac{1}{\tilde{\chi}^{2}} \tilde{\partial}_{\perp i}\left(B^{i(1)}+n^{l} h_{l}^{i(1)}\right)-\frac{2}{\tilde{\chi}^{3}}\left(B_{\|}^{(1)}+h_{\|}^{(1)}\right)\right.\right. \\
& \left.+\frac{1}{\tilde{\chi}} \tilde{\partial}_{\perp i}\left(B^{i(1) \prime}+n^{l} h_{l}^{i(1) \prime}\right)-\frac{2}{\tilde{\chi}^{2}}\left(B_{\|}^{(1) \prime}+h_{\|}^{(1) \prime}\right)\right] T^{(1)}-\left(B_{\perp i}^{(1) \prime}+n^{j} h_{j k}^{(1) \prime} \mathcal{P}_{i}^{k}\right) \tilde{\partial}_{\perp}^{i}\left(A^{(1)}-B_{\|}^{(1)}-\frac{1}{2} h_{\|}^{(1)}\right) \\
& -\left[\tilde{\partial}_{\perp}^{i}\left(A^{(1) \prime}-B_{\|}^{(1) \prime}-\frac{1}{2} h_{\|}^{(1) \prime}\right)+\frac{1}{\tilde{\chi}^{2}}\left(B_{\perp}^{i(1)}+n^{k} h_{k}^{j(1)} \mathcal{P}_{j}^{i}\right)+\frac{1}{\tilde{\chi}}\left(B_{\perp}^{i(1) \prime}+n^{j} h_{j}^{k(1) \prime} \mathcal{P}_{k}^{i}\right)\right] \tilde{\partial}_{\perp i} T^{(1)} \\
& -\left[\tilde{\partial}_{\perp m}\left(B^{m(1) \prime}+n^{l} h_{l}^{m(1) \prime}\right)-\frac{2}{\tilde{\chi}}\left(B_{\|}^{(1) \prime}+h_{\|}^{(1) \prime}\right)\right]\left(A^{(1)}-B_{\|}^{(1)}-\frac{1}{2} h_{\|}^{(1)}\right)+\left[\tilde{\nabla}_{\perp}^{2}\left(A^{(1)}-B_{\|}^{(1)}-\frac{1}{2} h_{\|}^{(1)}\right)\right. \\
& \left.+\frac{1}{\tilde{\chi}} \tilde{\partial}_{\perp m}\left(B^{m(1)}+n^{l} h_{l}^{m(1)}\right)-\frac{2}{\tilde{\chi}^{2}}\left(B_{\|}^{(1)}+h_{\|}^{(1)}\right)\right]\left(A^{(1)}+\frac{1}{2} h_{\|}^{(1)}-2 I^{(1)}\right)+\left[\partial_{\perp i}\left(A^{(1)}-B_{\|}^{(1)}-\frac{1}{2} h_{\|}^{(1)}\right)\right. \\
& \left.+\frac{1}{\bar{\chi}}\left(B_{\perp i}^{(1)}+n^{k} h_{k}^{j(1)} \mathcal{P}_{i j}\right)\right]\left[\tilde{\partial}_{\perp}^{i}\left(A^{(1)}+\frac{1}{2} h_{\|}^{(1)}\right)-2 \tilde{\partial}_{\perp}^{i} I^{(1)}\right]-\left[\tilde{\partial}_{\perp m}\left(B^{m(1)}+n^{l} h_{l}^{m(1)}\right)-\frac{2}{\tilde{\chi}}\left(B_{\|}^{(1)}+h_{\|}^{(1)}\right)\right] \\
& \times\left[\frac{\mathrm{d}}{\mathrm{d} \bar{\chi}}\left(A^{(1)}+\frac{1}{2} h_{\|}^{(1)}\right)+\left(A^{(1) \prime}-B_{\|}^{(1) \prime}-\frac{1}{2} h_{\|}^{(1) \prime}\right)\right]-\left(B_{\perp}^{i(1)}+n^{k} h_{k}^{j(1)} \mathcal{P}_{j}^{i}\right) \tilde{\partial}_{\perp i}\left[\frac{\mathrm{d}}{\mathrm{d} \bar{\chi}}\left(A^{(1)}+\frac{1}{2} h_{\|}^{(1)}\right)\right. \\
& \left.+\left(A^{(1) \prime}-B_{\|}^{(1) \prime}-\frac{1}{2} h_{\|}^{(1) \prime}\right)\right]+2 \frac{\mathrm{d}}{\mathrm{d} \tilde{\chi}}\left[\tilde{\partial}_{\perp m}\left(B^{m(1)}+n^{l} h_{l}^{m(1)}\right)-\frac{2}{\tilde{\chi}}\left(B_{\|}^{(1)}+h_{\|}^{(1)}\right)\right] \kappa^{(1)} \\
& +\frac{2}{\tilde{\chi}}\left[-\frac{1}{\tilde{\chi}}\left(A^{(1)}-B_{\|}^{(1)}-\frac{1}{2} h_{\|}^{(1)}\right)+\frac{\mathrm{d}}{\mathrm{d} \tilde{\chi}}\left(A^{(1)}-B_{\|}^{(1)}-\frac{1}{2} h_{\|}^{(1)}\right)\right] \kappa^{(1)}+2\left[\frac{\mathrm{d}}{\mathrm{d} \tilde{\chi}}\left(B_{\perp}^{i(1)}+n^{k} h_{k}^{j(1)} \mathcal{P}_{j}^{i}\right)\right. \\
& \left.-\frac{1}{\tilde{\chi}}\left(B_{\perp}^{i(1)}+n^{k} h_{k}^{j(1)} \mathcal{P}_{j}^{i}\right)\right] \tilde{\partial}_{\perp i} \kappa^{(1)}-\left[-\tilde{\partial}_{\perp i} \tilde{\partial}_{\perp m}\left(B^{m(1)}+n^{k} h_{k}^{m(1)}\right)-\frac{1}{\tilde{\chi}} \tilde{\partial}_{\perp i}\left(A^{(1)}-B_{\|}^{(1)}-\frac{1}{2} h_{\|}^{(1)}\right)\right. \\
& \left.+\frac{1}{\tilde{\chi}} \tilde{\partial}_{\perp i}\left(B_{\|}^{(1)}+h_{\|}^{(1)}\right)+\frac{1}{\tilde{\chi}^{2}}\left(B_{\perp i}^{(1)}+n^{k} h_{k}^{j(1)} \mathcal{P}_{i j}\right)+\frac{1}{\tilde{\chi}} \tilde{\partial}_{\perp m} h_{i}^{m(1)}\right]\left(B_{\perp}^{i(1)}+n^{k} h_{k}^{j(1)} \mathcal{P}_{j}^{i}-2 S_{\perp}^{i(1)}\right) \\
& +\left[\tilde{\partial}_{\perp}^{j}\left(B^{m(1)}+n^{k} h_{k}^{m(1)}\right)-\frac{1}{\tilde{\chi}} h^{j m(1)}\right] \tilde{\partial}_{\perp m}\left(B_{j \perp}^{(1)}+n^{l} h_{l}^{p(1)} \mathcal{P}_{p j}-2 S_{\perp j}^{(1)}\right)-\left(B_{\perp i}^{(1)}+n^{k} h_{k}^{j(1)} \mathcal{P}_{i j}\right) \\
& \times\left[-\tilde{\partial}_{\perp}^{i} \tilde{\partial}_{\perp m}\left(B^{m(1)}+n^{l} h_{l}^{m(1)}\right)+\frac{2}{\tilde{\chi}} \tilde{\partial}_{\perp}^{i}\left(B_{\|}^{(1)}+h_{\|}^{(1)}\right)+2 \tilde{\partial}_{\perp}^{i} \tilde{\partial}_{\perp m} S_{\perp}^{m(1)}\right]+\left[-\tilde{\partial}_{\perp m}\left(B^{m(1)}+n^{l} h_{l}^{m(1)}\right)\right. \\
& \left.-\frac{1}{\tilde{\chi}}\left(A^{(1)}-B_{\|}^{(1)}-\frac{1}{2} h_{\|}^{(1)}\right)+\frac{2}{\tilde{\chi}}\left(B_{\|}^{(1)}+h_{\|}^{(1)}\right)\right]\left[-\tilde{\partial}_{\perp m}\left(B^{m(1)}+n^{l} h_{l}^{m(1)}\right)+\frac{2}{\tilde{\chi}}\left(B_{\|}^{(1)}+h_{\|}^{(1)}\right)+2 \tilde{\partial}_{\perp m} S_{\perp}^{m(1)}\right] \\
& +\left[+\tilde{\partial}_{\perp i} \tilde{\nabla}_{\perp}^{2}\left(A^{(1)}-B_{\|}^{(1)}-\frac{1}{2} h_{\|}^{(1)}\right)+\frac{1}{\tilde{\chi}} \tilde{\partial}_{\perp i}\left(A^{(1) \prime}-B_{\|}^{(1) \prime}-\frac{1}{2} h_{\|}^{(1) \prime}\right)+\frac{2}{\tilde{\chi}^{3}}\left(B_{\perp i}^{(1)}+n^{k} h_{k}^{j(1)} \mathcal{P}_{i j}\right)\right. \\
& -\frac{3}{\tilde{\chi}^{2}} \tilde{\partial}_{\perp i}\left(B_{\|}^{(1)}+h_{\|}^{(1)}\right)+\frac{1}{\tilde{\chi}} \tilde{\partial}_{\perp i} \tilde{\partial}_{\perp m}\left(B^{m(1)}+n^{k} h_{k}^{m(1)}\right)+\frac{2}{\tilde{\chi}^{2}} \tilde{\partial}_{\perp i}\left(A^{(1)}-B_{\|}^{(1)}-\frac{1}{2} h_{\|}^{(1)}\right)-\frac{1}{\tilde{\chi}^{2}} \tilde{\partial}_{\perp k} h_{i}^{k(1)} \\
& \left.+\frac{1}{\tilde{\chi}} \mathcal{P}_{i}^{l} \tilde{\nabla}_{\perp}^{2}\left(B_{l}^{(1)}+n^{m} h_{l m}^{(1)}\right)+\frac{2}{\tilde{\chi}^{3}} n^{k} h_{k}^{j(1)} \mathcal{P}_{i j}\right] \delta x_{\perp}^{i(1)}+\left[-\tilde{\partial}_{\perp}^{(j} \tilde{\partial}_{\perp}^{m)}\left(A^{(1)}-B_{\|}^{(1)}-\frac{1}{2} h_{\|}^{(1)}\right)\right. \\
& -\frac{1}{\tilde{\chi}} \mathcal{P}^{j m}\left(A^{(1) \prime}-B_{\|}^{(1) \prime}-\frac{1}{2} h_{\|}^{(1) \prime}\right)-\frac{1}{\tilde{\chi}^{2}} \mathcal{P}^{j m}\left(A^{(1)}-B_{\|}^{(1)}-\frac{1}{2} h_{\|}^{(1)}\right)-\frac{1}{\tilde{\chi}} \tilde{\partial}_{\perp}^{j}\left(B^{m(1)}+n^{k} h_{k}^{l(1)} \mathcal{P}_{l}^{m}\right) \\
& \left.\left.-\frac{1}{\tilde{\chi}} n^{[j} \partial_{\perp}^{m]}\left(A^{(1)}-B_{\|}^{(1)}-\frac{1}{2} h_{\|}^{(1)}\right)-\frac{1}{\tilde{\chi}} \mathcal{P}^{k j} \tilde{\partial}_{\perp}^{m}\left(B_{k}^{(1)}+n^{l} h_{k l}^{(1)}\right)+\frac{1}{\tilde{\chi}^{2}} \mathcal{P}_{l}^{m} h^{l j(1)}\right]\left(\gamma_{m j}^{(1)}+\mathcal{P}_{m j} \kappa^{(1)}+\theta_{m j}^{(1)}\right)\right\}
\end{aligned}
$$

and 


$$
\begin{aligned}
& \kappa_{3 \text { post-Born }}^{(2)}=-\left(A_{o}^{(1)}-B_{\| o}^{(1)}-\frac{1}{2} h_{\| o}^{(1)}\right)\left(2 v_{\| o}^{(1)}+\frac{3}{2} h_{\| o}^{(1)}-\frac{1}{2} h_{j o}^{j(1)}\right)+\left(2 B_{\| o}^{(1)}+3 h_{\| o}^{(1)}-h_{j o}^{j(1)}\right)\left(3 v_{\| o}^{(1)}+2 h_{\| o}^{(1)}-\frac{1}{2} h_{j o}^{j(1)}\right) \\
& -\left(B_{\perp o}^{i(1)}+n^{k} h_{k o}^{j(1)} \mathcal{P}_{j}^{i}\right)\left(4 v_{\perp i o}^{(1)}+\frac{9}{2} n^{l} h_{l o}^{m(1)} \mathcal{P}_{m i}\right)+\left(B_{\| o}^{(1)}-v_{\| o}^{(1)}+\frac{1}{2} h_{\| o}^{(1)}\right) \int_{0}^{\bar{\chi}} \mathrm{d} \tilde{\chi} \frac{(\bar{\chi}-\tilde{\chi})}{\bar{\chi}} \\
& \times\left[\tilde{\partial}_{\perp}^{m}\left(B^{m(1)}+n^{k} h_{k}^{m(1)}\right)-\frac{1}{\tilde{\chi}} \mathcal{P}_{m j} h^{j m(1)}\right]+2\left(-B_{\| o}^{(1)}+v_{\| o}^{(1)}-\frac{3}{4} h_{\| o}^{(1)}+\frac{1}{4} h_{m o}^{m(1)}\right) \int_{0}^{\bar{\chi}} \mathrm{d} \tilde{\chi} \frac{(\bar{\chi}-\tilde{\chi})}{\bar{\chi}} \\
& \times\left[-\tilde{\partial}_{\perp m}\left(B^{m(1)}+n^{l} h_{l}^{m(1)}\right)-\frac{1}{\tilde{\chi}}\left(A^{(1)}-B_{\|}^{(1)}-\frac{1}{2} h_{\|}^{(1)}\right)+\frac{2}{\tilde{\chi}}\left(B_{\|}^{(1)}+h_{\|}^{(1)}\right)\right]+\left(A_{o}^{(1)}-v_{\| o}^{(1)}\right) \\
& \times\left\{\bar{\chi}\left[\partial_{\perp i}\left(B^{i(1)}+n^{k} h_{k}^{i(1)}\right)-\frac{2}{\tilde{\chi}}\left(B_{\|}^{(1)}+h_{\|}^{(1)}\right)\right]-2 \int_{0}^{\bar{\chi}} \mathrm{d} \tilde{\chi} \frac{\tilde{\chi}}{\bar{\chi}}\left[\tilde{\partial}_{\perp m}\left(B^{m(1)}+n^{l} h_{l}^{m(1)}\right)-\frac{2}{\tilde{\chi}}\left(B_{\|}^{(1)}+h_{\|}^{(1)}\right)\right]\right. \\
& \left.-\int_{0}^{\bar{\chi}} \mathrm{d} \tilde{\chi}(\bar{\chi}-\tilde{\chi}) \frac{\tilde{\chi}}{\bar{\chi}}\left[\tilde{\nabla}_{\perp}^{2}\left(A^{(1)}-B_{\|}^{(1)}-\frac{1}{2} h_{\|}^{(1)}\right)+\frac{1}{\tilde{\chi}} \tilde{\partial}_{\perp m}\left(B^{m(1)}+n^{l} h_{l}^{m(1)}\right)-\frac{2}{\tilde{\chi}^{2}}\left(B_{\|}^{(1)}+h_{\|}^{(1)}\right)\right]\right\} \\
& +v_{\perp i o}^{(1)}\left\{-\left(B_{\perp}^{(1) i}+n^{j} h_{j}^{k(1)} \mathcal{P}_{k}^{i}\right)+\frac{2}{\bar{\chi}} \int_{0}^{\bar{\chi}} \mathrm{d} \tilde{\chi}\left(B_{\perp}^{(1) i}+n^{j} h_{j}^{k(1)} \mathcal{P}_{k}^{i}\right)\right. \\
& \left.+\frac{1}{\bar{\chi}} \int_{0}^{\bar{\chi}} \mathrm{d} \tilde{\chi}(\bar{\chi}-\tilde{\chi})\left[\partial_{\perp}^{i}\left(A^{(1)}-B_{\|}^{(1)}-\frac{1}{2} h_{\|}^{(1)}\right)+\frac{1}{\bar{\chi}}\left(B_{\perp}^{i(1)}+n^{k} h_{k}^{j(1)} \mathcal{P}_{j}^{i}\right)\right]\right\} \\
& +\left(B_{\perp o}^{i(1)}-v_{\perp o}^{i(1)}+\frac{1}{2} n^{k} h_{k o}^{j(1)} \mathcal{P}_{j}^{i}\right) \int_{0}^{\bar{\chi}} \mathrm{d} \tilde{\chi}(\bar{\chi}-\tilde{\chi}) \frac{\tilde{\chi}}{\bar{\chi}}\left[-\tilde{\partial}_{\perp i} \tilde{\partial}_{\perp m}\left(B^{m(1)}+n^{k} h_{k}^{m(1)}\right)\right. \\
& \left.\left.-\frac{1}{\tilde{\chi}} \tilde{\partial}_{\perp i}\left(A^{(1)}-B_{\|}^{(1)}-\frac{1}{2} h_{\|}^{(1)}\right)+\frac{1}{\tilde{\chi}} \tilde{\partial}_{\perp i}\left(B_{\|}^{(1)}+h_{\|}^{(1)}\right)+\frac{1}{\tilde{\chi}^{2}}\left(B_{\perp i}^{(1)}+n^{k} h_{k}^{j(1)} \mathcal{P}_{i j}\right)+\frac{1}{\tilde{\chi}} \tilde{\partial}_{\perp m} h_{i}^{m(1)}\right]\right] \\
& -2\left(B_{\perp o}^{i(1)}-v_{\perp o}^{i(1)}-\frac{3}{2} n^{k} h_{k o}^{m(1)} \mathcal{P}_{m}^{i}\right) \int_{0}^{\bar{\chi}} \mathrm{d} \tilde{\chi} \frac{(\bar{\chi}-\tilde{\chi})}{\bar{\chi} \tilde{\chi}}\left(B_{\perp i}^{(1)}+n^{k} h_{k}^{j(1)} \mathcal{P}_{i j}\right) \\
& -\frac{1}{2} \mathcal{P}_{m}^{k} \mathcal{P}_{j}^{l} h_{l k o}^{(1)} \int_{0}^{\bar{\chi}} \mathrm{d} \tilde{\chi} \frac{(\bar{\chi}-\tilde{\chi})}{\bar{\chi}}\left[\tilde{\partial}_{\perp}^{j}\left(B^{m(1)}+n^{k} h_{k}^{m(1)}\right)-\frac{1}{\tilde{\chi}} h^{j m(1)}\right] .
\end{aligned}
$$

Finally, in order to obtain $\Delta_{g}^{(2)}$ [see Eq. 77$]$ ], we need the following

$$
\begin{gathered}
\hat{g}_{\mu}^{\mu(2)}-\hat{g}_{\mu}^{\nu(1)} \hat{g}_{\nu}^{\mu(1)}=2 A^{(2)}+h_{i}^{i(2)}-4\left(A^{(1)}\right)^{2}+2 B_{i}^{(1)} B^{i(1)}-h_{i}^{k(1)} h_{k}^{i(1)}, \\
E_{\hat{0}}^{0(2)}+E_{\hat{0}}^{\|(2)}=-A^{(2)}+v_{\|}^{(2)}+3\left(A^{(1)}\right)^{2}+v_{i}^{(1)} v^{i(1)}-2 v_{i}^{(1)} B^{i(1)}, \\
\frac{1}{\mathcal{H}} \hat{g}_{\mu}^{\mu(1) \prime} \Delta \ln a^{(1)}+\left(\partial_{\|} \hat{g}_{\mu}^{\mu(1)}\right) \Delta x_{\|}^{(1)}=-\frac{1}{\mathcal{H}} \Delta \ln a^{(1)} \frac{\mathrm{d}}{\mathrm{d} \bar{\chi}}\left(\hat{g}_{\mu}^{\mu(1)}\right)-\left(\partial_{\|} \hat{g}_{\mu}^{\mu(1)}\right) T^{(1)}\left(2 A^{(1)}+h_{i}^{i(1)}\right) \Delta \ln a^{(1)}-\partial_{\|}\left(2 A^{(1)}+h_{i}^{i(1)}\right) T^{(1)}, \\
\left(\partial_{\perp i} \hat{g}_{\mu}^{\mu(1)}\right) \Delta x_{\perp}^{i(1)}=\partial_{\perp i}\left(2 A^{(1)}+h_{i}^{i(1)}\right) \delta x_{\perp}^{i(1)}=\bar{\chi}\left(B_{\perp o}^{i(1)}-v_{\perp o}^{i(1)}+\frac{1}{2} n^{k} h_{k o}^{j(1)} \mathcal{P}_{j}^{i}\right) \partial_{\perp i}\left(2 A^{(1)}+h_{i}^{i(1)}\right) \\
-\partial_{\perp i}\left(2 A^{(1)}+h_{i}^{i(1)}\right) \int_{0}^{\bar{\chi}} \mathrm{d} \tilde{\chi}\left\{\left(B_{\perp}^{i(1)}+n^{k} h_{k}^{j(1)} \mathcal{P}_{j}^{i}\right)+(\bar{\chi}-\tilde{\chi})\right. \\
\left.\times\left[\tilde{\partial}_{\perp}^{i}\left(A^{(1)}-B_{\|}^{(1)}-\frac{1}{2} h_{\|}^{(1)}\right)+\frac{1}{\tilde{\chi}}\left(B_{\perp}^{i(1)}+n^{k} h_{k j}^{(1)} \mathcal{P}^{i j}\right)\right]\right\},
\end{gathered}
$$




$$
\begin{aligned}
& \frac{1}{\mathcal{H}} \delta_{g}^{(1) \prime} \Delta \ln a^{(1)}+\left(\partial_{\|} \delta_{g}^{(1)}\right) \Delta x_{\|}^{(1)}=-\frac{1}{\mathcal{H}} \frac{\mathrm{d}}{\mathrm{d} \bar{\chi}}\left(\delta_{g}^{(1)}\right) \Delta \ln a^{(1)}-\left(\partial_{\|} \delta_{g}^{(1)}\right) T^{(1)}, \\
& \Delta x_{\perp i}^{(1)} \partial_{\perp}^{i} \delta_{g}^{(1)}=\delta x_{\perp i}^{(1)} \partial_{\perp}^{i} \delta_{g}^{(1)}=\bar{\chi}\left(B_{\perp o}^{i(1)}-v_{\perp o}^{i(1)}+\frac{1}{2} n^{k} h_{k o}^{j(1)} \mathcal{P}_{j}^{i}\right) \partial_{\perp i} \delta_{g}^{(1)}-\left(\partial_{\perp i} \delta_{g}^{(1)}\right) \int_{0}^{\bar{\chi}} \mathrm{d} \tilde{\chi}\left\{\left(B_{\perp}^{i(1)}+n^{k} h_{k}^{j(1)} \mathcal{P}_{j}^{i}\right)\right. \\
& \left.+(\bar{\chi}-\tilde{\chi})\left[\tilde{\partial}_{\perp}^{i}\left(A^{(1)}-B_{\|}^{(1)}-\frac{1}{2} h_{\|}^{(1)}\right)+\frac{1}{\tilde{\chi}}\left(B_{\perp}^{i(1)}+n^{k} h_{k j}^{(1)} \mathcal{P}^{i j}\right)\right]\right\} \\
& -\frac{1}{\bar{\chi}^{2}}\left(\Delta x_{\|}^{(1)}\right)^{2}=-\frac{1}{\bar{\chi}^{2}}\left(T^{(1)}\right)^{2}-\frac{1}{\bar{\chi}^{2} \mathcal{H}^{2}}\left(\Delta \ln a^{(1)}\right)^{2}-\frac{2}{\bar{\chi}^{2} \mathcal{H}} \Delta \ln a^{(1)} T^{(1)}, \\
& \frac{2}{\bar{\chi}} \Delta x_{\|}^{(1)} \kappa^{(1)}=-\frac{2}{\bar{\chi} \mathcal{H}} \Delta \ln a^{(1)} \kappa^{(1)}-\frac{2}{\bar{\chi}} T^{(1)} \kappa^{(1)}, \\
& -\frac{1}{2}\left(\partial_{\perp j} \Delta x_{\perp}^{i(1)}\right)\left(\partial_{\perp i} \Delta x_{\perp}^{j(1)}\right)=-\left(v_{\| o}^{(1)}\right)^{2}-\left(A_{o}^{(1)}\right)^{2}-\frac{1}{2} v_{\| o}^{(1)} h_{i o}^{i(1)}+\frac{1}{2} v_{\| o}^{(1)} h_{\| o}^{(1)}-\frac{1}{8} \mathcal{P}_{p}^{m} \mathcal{P}_{n}^{k} h_{m o}^{n(1)} h_{k o}^{p(1)} \\
& +2 A_{o}^{(1)} v_{\| o}^{(1)}+\frac{1}{2} A_{o}^{(1)} h_{i o}^{i(1)}-\frac{1}{2} A_{o}^{(1)} h_{\| o}^{(1)}+\left(2 A_{o}^{(1)}-2 v_{\| o}^{(1)}-\frac{1}{2} h_{i o}^{i(1)}+\frac{1}{2} h_{\| o}^{(1)}\right)\left(A^{(1)}-B_{\|}^{(1)}-\frac{1}{2} h_{\|}^{(1)}-2 I^{(1)}\right) \\
& +\left(\mathcal{P}_{j}^{i} A_{o}^{(1)}-\mathcal{P}_{j}^{i} v_{\| o}^{(1)}-\frac{1}{2} \mathcal{P}_{j}^{k} \mathcal{P}_{p}^{i} h_{k o}^{p(1)}\right) \int_{0}^{\bar{\chi}} \mathrm{d} \tilde{\chi}\left\{\frac{1}{\tilde{\chi}} \mathcal{P}_{i}^{j} B_{\|}^{(1)}-\mathcal{P}_{m}^{j} \tilde{\partial}_{\perp i} B^{m(1)}-\frac{1}{\tilde{\chi}} \mathcal{P}_{i}^{n} \mathcal{P}_{m}^{j} h_{n}^{m(1)}+\frac{1}{\tilde{\chi}} \mathcal{P}_{i}^{j} h_{\|}^{(1)}-n^{m} \mathcal{P}_{n}^{j} \tilde{\partial}_{\perp i} h_{m}^{n(1)}\right. \\
& \left.-\frac{\tilde{\chi}}{\bar{\chi}}\left[\mathcal{P}_{i}^{j} \tilde{\partial}_{\|}+(\bar{\chi}-\tilde{\chi}) \mathcal{P}_{i}^{n} \mathcal{P}^{j m} \tilde{\partial}_{m} \tilde{\partial}_{n}\right]\left(A^{(1)}-B_{\|}^{(1)}-\frac{1}{2} h_{\|}^{(1)}\right)\right\}-\left(A^{(1)}-B_{\|}^{(1)}-\frac{1}{2} h_{\|}^{(1)}-2 I^{(1)}\right)^{2} \\
& -\left(A^{(1)}-B_{\|}^{(1)}-\frac{1}{2} h_{\|}^{(1)}-2 I^{(1)}\right) \int_{0}^{\bar{\chi}} \mathrm{d} \tilde{\chi}\left\{\frac{2}{\tilde{\chi}} B_{\|}^{(1)}-\tilde{\partial}_{\perp m} B^{m(1)}-\frac{1}{\tilde{\chi}} h_{i}^{i(1)}+\frac{3}{\tilde{\chi}} h_{\|}^{(1)}-n^{m} \tilde{\partial}_{\perp n} h_{m}^{n(1)}\right. \\
& \left.-\frac{\tilde{\chi}}{\bar{\chi}}\left[2 \tilde{\partial}_{\|}+(\bar{\chi}-\tilde{\chi}) \mathcal{P}^{m n} \tilde{\partial}_{m} \tilde{\partial}_{n}\right]\left(A^{(1)}-B_{\|}^{(1)}-\frac{1}{2} h_{\|}^{(1)}\right)\right\}-\frac{1}{2} \int_{0}^{\bar{\chi}} \mathrm{d} \tilde{\chi}\left\{\frac{1}{\tilde{\chi}} \mathcal{P}_{j}^{i} B_{\|}^{(1)}-\mathcal{P}_{p}^{i} \tilde{\partial}_{\perp j} B^{p(1)}-\frac{1}{\tilde{\chi}} \mathcal{P}_{j}^{p} \mathcal{P}_{q}^{i} h_{p}^{q(1)}\right. \\
& \left.+\frac{1}{\tilde{\chi}} \mathcal{P}_{j}^{i} h_{\|}^{(1)}-n^{p} \mathcal{P}_{q}^{i} \tilde{\partial}_{\perp j} h_{p}^{q(1)}-\frac{\tilde{\chi}}{\bar{\chi}}\left[\mathcal{P}_{j}^{i} \tilde{\partial}_{\|}+(\bar{\chi}-\tilde{\chi}) \mathcal{P}_{j}^{p} \mathcal{P}^{i q} \tilde{\partial}_{q} \tilde{\partial}_{p}\right]\left(A^{(1)}-B_{\|}^{(1)}-\frac{1}{2} h_{\|}^{(1)}\right)\right\} \int_{0}^{\bar{\chi}} \mathrm{d} \tilde{\chi}\left\{\frac{1}{\tilde{\chi}} \mathcal{P}_{i}^{j} B_{\|}^{(1)}\right. \\
& -\mathcal{P}_{m}^{j} \tilde{\partial}_{\perp i} B^{m(1)}-\frac{1}{\tilde{\chi}} \mathcal{P}_{i}^{n} \mathcal{P}_{m}^{j} h_{n}^{m(1)}+\frac{1}{\tilde{\chi}} \mathcal{P}_{i}^{j} h_{\|}^{(1)}-n^{m} \mathcal{P}_{n}^{j} \tilde{\partial}_{\perp i} h_{m}^{n(1)}-\frac{\tilde{\chi}}{\bar{\chi}}\left[\mathcal{P}_{i}^{j} \tilde{\partial}_{\|}+(\bar{\chi}-\tilde{\chi}) \mathcal{P}_{i}^{n} \mathcal{P}^{j m} \tilde{\partial}_{m} \tilde{\partial}_{n}\right] \\
& \left.\times\left(A^{(1)}-B_{\|}^{(1)}-\frac{1}{2} h_{\|}^{(1)}\right)\right\}, \\
& \left(\frac{1}{\bar{\chi}} \Delta x_{\perp i}^{(1)}-\partial_{\perp i} \Delta x_{\|}^{(1)}\right) \partial_{\|} \Delta x_{\perp}^{i(1)}=\left(\frac{1}{\bar{\chi}} \delta x_{\perp i}^{(1)}-\partial_{\perp i} \Delta x_{\|}^{(1)}\right) \delta n_{\perp}^{i(1)}=\left(B_{\perp i o}^{(1)}-v_{\perp i o}^{(1)}+\frac{1}{2} n^{k} h_{k o}^{j(1)} \mathcal{P}_{i j}\right) \\
& \times\left(B_{\perp o}^{i(1)}-v_{\perp o}^{i(1)}+\frac{1}{2} n^{k} h_{k o}^{j(1)} \mathcal{P}_{j}^{i}\right)+\left(B_{\perp i o}^{(1)}-v_{\perp i o}^{(1)}+\frac{1}{2} n^{k} h_{k o}^{j(1)} \mathcal{P}_{i j}\right)\left\{-\left(B_{\perp}^{i(1)}+n^{k} h_{k}^{j(1)} \mathcal{P}_{j}^{i}\right)+2 S_{\perp}^{i(1)}\right. \\
& \left.+\partial_{\perp i}\left(\frac{1}{\mathcal{H}} \Delta \ln a^{(1)}+T^{(1)}\right)-\frac{1}{\bar{\chi}} \int_{0}^{\bar{\chi}} \mathrm{d} \tilde{\chi}\left[\frac{\bar{\chi}}{\tilde{\chi}}\left(B_{\perp}^{i(1)}+n^{k} h_{k}^{j(1)} \mathcal{P}_{j}^{i}\right)+(\bar{\chi}-\tilde{\chi}) \tilde{\partial}_{\perp}^{i}\left(A^{(1)}-B_{\|}^{(1)}-\frac{1}{2} h_{\|}^{(1)}\right)\right]\right\} \\
& +\left[-\left(B_{\perp}^{i(1)}+n^{k} h_{k}^{j(1)} \mathcal{P}_{j}^{i}\right)+2 S_{\perp}^{i(1)}\right]\left\{-\frac{1}{\bar{\chi}} \int_{0}^{\bar{\chi}} \mathrm{d} \tilde{\chi}\left[\frac{\bar{\chi}}{\tilde{\chi}}\left(B_{\perp}^{i(1)}+n^{k} h_{k}^{j(1)} \mathcal{P}_{j}^{i}\right)+(\bar{\chi}-\tilde{\chi}) \tilde{\partial}_{\perp}^{i}\left(A^{(1)}-B_{\|}^{(1)}-\frac{1}{2} h_{\|}^{(1)}\right)\right]\right. \\
& \left.+\partial_{\perp i}\left(\frac{1}{\mathcal{H}} \Delta \ln a^{(1)}+T^{(1)}\right)\right\} \\
& \frac{1}{\mathcal{H}}\left(E_{\hat{0}}^{0(1)}+E_{\hat{0}}^{\|(1)}\right)^{\prime} \Delta \ln a^{(1)}+\partial_{\|}\left(E_{\hat{0}}^{0(1)}+E_{\hat{0}}^{\|(1)}\right) \Delta x_{\|}^{(1)}=\frac{1}{\mathcal{H}} \frac{\mathrm{d}}{\mathrm{d} \bar{\chi}}\left(A^{(1)}-v_{\|}^{(1)}\right) \Delta \ln a^{(1)}+\partial_{\|}\left(A^{(1)}-v_{\|}^{(1)}\right) T^{(1)}
\end{aligned}
$$




$$
\begin{gathered}
\partial_{\perp i}\left(E_{\hat{0}}^{0(1)}+E_{\hat{0}}^{\|(1)}\right) \Delta x_{\perp}^{i(1)}=-\bar{\chi}\left(B_{\perp o}^{i(1)}-v_{\perp o}^{i(1)}+\frac{1}{2} n^{k} h_{k o}^{j(1)} \mathcal{P}_{j}^{i}\right) \partial_{\perp i}\left(A^{(1)}-v_{\|}^{(1)}\right)+\partial_{\perp i}\left(A^{(1)}-v_{\|}^{(1)}\right) \\
\times \int_{0}^{\bar{\chi}} \mathrm{d} \tilde{\chi}\left\{\left(B_{\perp}^{i(1)}+n^{k} h_{k}^{j(1)} \mathcal{P}_{j}^{i}\right)+(\bar{\chi}-\tilde{\chi})\left[\tilde{\partial}_{\perp}^{i}\left(A^{(1)}-B_{\|}^{(1)}-\frac{1}{2} h_{\|}^{(1)}\right)+\frac{1}{\tilde{\chi}}\left(B_{\perp}^{i(1)}+n^{k} h_{k j}^{(1)} \mathcal{P}^{i j}\right)\right]\right\}, \quad(153) \\
-\left(\delta n_{\|}^{(1)}+\delta \nu^{(1)}\right) E_{\hat{0}}^{\|(1)}=v_{\|}^{(1)}\left(\frac{\mathrm{d} T}{\mathrm{~d} \bar{\chi}}\right)^{(1)}=-v_{\|}^{(1)}\left(A^{(1)}-B_{\|}^{(1)}-\frac{1}{2} h_{\|}^{(1)}\right), \\
-E_{\hat{0}}^{\perp i(1)} \partial_{\perp i}\left(\Delta x^{0(1)}+\Delta x_{\|}^{(1)}\right)=-E_{\hat{0}}^{\perp i(1)} \partial_{\perp i}\left(\delta x^{0(1)}+\delta x_{\|}^{(1)}\right)=v_{\perp}^{i(1)} \partial_{\perp i} T^{(1)}, \\
-\frac{1}{2}\left(\partial_{\|} \Delta x_{\|}^{(1)}\right)^{2}=-\frac{1}{2}\left(A^{(1)}-B_{\|}^{(1)}-\frac{1}{2} h_{\|}^{(1)}\right)^{2}-\frac{1}{2 \mathcal{H}^{2}}\left[\frac{\mathrm{d}}{\mathrm{d} \bar{\chi}}\left(A^{(1)}-v_{\|}^{(1)}\right)+\left(A^{(1) \prime}-B_{\|}^{(1) \prime}-\frac{1}{2} h_{\|}^{(1) \prime}\right)\right]^{2} \\
-\frac{1}{\mathcal{H}}\left(A^{(1)}-B_{\|}^{(1)}-\frac{1}{2} h_{\|}^{(1)}\right)\left[\frac{\mathrm{d}}{\mathrm{d} \bar{\chi}}\left(A^{(1)}-v_{\|}^{(1)}\right)+\left(A^{(1) \prime}-B_{\|}^{(1) \prime}-\frac{1}{2} h_{\|}^{(1) \prime}\right)\right]+\frac{\mathcal{H}^{\prime}}{\mathcal{H}^{2}}\left(A^{(1)}-B_{\|}^{(1)}-\frac{1}{2} h_{\|}^{(1)}\right) \Delta \ln a^{(1)} \\
+\frac{\mathcal{H}^{\prime}}{\mathcal{H}^{3}}\left[\frac{\mathrm{d}}{\mathrm{d} \bar{\chi}}\left(A^{(1)}-v_{\|}^{(1)}\right)+\left(A^{(1) \prime}-B_{\|}^{(1) \prime}-\frac{1}{2} h_{\|}^{(1) \prime}\right)\right] \Delta \ln a^{(1)}-\frac{1}{2}\left(\frac{\mathcal{H}^{\prime}}{\mathcal{H}^{2}}\right)^{2}\left(\Delta \ln a^{(1)}\right)^{2}, \\
-\frac{1}{2}\left(E_{\hat{0}}^{0(1)}+E_{\hat{0}}^{\|(1)}\right)^{2}=-\frac{1}{2}\left(A^{(1)}-v_{\|}^{(1)}\right)^{2} \cdot
\end{gathered}
$$


Putting together the above relations we obtain finally

$$
\begin{aligned}
& \Delta_{g}^{(2)}=\delta_{g}^{(2)}+b_{e} \Delta \ln a^{(2)}+\partial_{\|} \Delta x_{\|}^{(2)}+\frac{2}{\bar{\chi}} \Delta x_{\|}^{(2)}-2 \kappa^{(2)}+A^{(2)}+v_{\|}^{(2)}+\frac{1}{2} h_{i}^{i(2)}+\left(\Delta_{g}^{(1)}\right)^{2}-3\left(A^{(1)}\right)^{2}+6 A^{(1)} B_{\|}^{(1)} \\
& +3 A^{(1)} h_{\|}^{(1)}-3 B_{\|}^{(1)} h_{\|}^{(1)}+v_{\perp i}^{(1)} v_{\perp}^{i(1)}-2 v_{\perp i}^{(1)} B_{\perp}^{i(1)}-2\left(B_{\|}^{(1)}\right)^{2}+B_{\perp i}^{(1)} B_{\perp}^{i(1)}-\frac{1}{2} h_{i}^{k(1)} h_{k}^{i(1)}-\frac{3}{4}\left(h_{\|}^{(1)}\right)^{2}-8\left(I^{(1)}\right)^{2} \\
& +8 A^{(1)} I^{(1)}-8 B_{\|}^{(1)} I^{(1)}-4 h_{\|}^{(1)} I^{(1)}-\left(\delta_{g}^{(1)}\right)^{2}+v_{\|}^{(1)} h_{\|}^{(1)}-\frac{1}{\mathcal{H}^{2}}\left[\frac{\mathrm{d}}{\mathrm{d} \bar{\chi}}\left(A^{(1)}-v_{\|}^{(1)}\right)+\left(A^{(1) \prime}-B_{\|}^{(1) \prime}-\frac{1}{2} h_{\|}^{(1) \prime}\right)\right]^{2} \\
& -\frac{2}{\mathcal{H}}\left(A^{(1)}-B_{\|}^{(1)}-\frac{1}{2} h_{\|}^{(1)}\right)\left[\frac{\mathrm{d}}{\mathrm{d} \bar{\chi}}\left(A^{(1)}-v_{\|}^{(1)}\right)+\left(A^{(1) \prime}-B_{\|}^{(1) \prime}-\frac{1}{2} h_{\|}^{(1) \prime}\right)\right]-\frac{1}{\mathcal{H}} \frac{\mathrm{d}}{\mathrm{d} \bar{\chi}}\left(2 v_{\|}^{(1)}+h_{i}^{i(1)}+2 \delta_{g}^{(1)}\right) \Delta \ln a^{(1)} \\
& -\partial_{\|}\left(2 v_{\|}^{(1)}+h_{i}^{i(1)}+2 \delta_{g}^{(1)}\right) T^{(1)}-\frac{4}{\bar{\chi}^{2} \mathcal{H}} \Delta \ln a^{(1)} T^{(1)}+2 v_{\perp}^{i(1)} \partial_{\perp i} T^{(1)}-\frac{4}{\bar{\chi} \mathcal{H}} \Delta \ln a^{(1)} \kappa^{(1)}-\frac{4}{\bar{\chi}} T^{(1)} \kappa^{(1)} \\
& +\left[-b_{e}+\frac{\mathrm{d} \ln b_{e}}{\mathrm{~d} \ln \bar{a}}-\left(\frac{\mathcal{H}^{\prime}}{\mathcal{H}^{2}}\right)^{2}-\frac{2}{\bar{\chi}^{2} \mathcal{H}^{2}}\right]\left(\Delta \ln a^{(1)}\right)^{2}-\frac{2}{\bar{\chi}^{2}}\left(T^{(1)}\right)^{2}+2 \frac{\mathcal{H}^{\prime}}{\mathcal{H}^{2}}\left(A^{(1)}-B_{\|}^{(1)}-\frac{1}{2} h_{\|}^{(1)}\right) \Delta \ln a^{(1)} \\
& +2 \frac{\mathcal{H}^{\prime}}{\mathcal{H}^{3}}\left[\frac{\mathrm{d}}{\mathrm{d} \bar{\chi}}\left(A^{(1)}-v_{\|}^{(1)}\right)+\left(A^{(1) \prime}-B_{\|}^{(1) \prime}-\frac{1}{2} h_{\|}^{(1) \prime}\right)\right] \Delta \ln a^{(1)}+2\left[-\left(B_{\perp}^{i(1)}+n^{k} h_{k}^{j(1)} \mathcal{P}_{j}^{i}\right)+2 S_{\perp}^{i(1)}\right] \\
& \times \partial_{\perp i}\left(\frac{1}{\mathcal{H}} \Delta \ln a^{(1)}+T^{(1)}\right)-\left[-\frac{2}{\bar{\chi}}\left(B_{\perp}^{i(1)}+n^{k} h_{k}^{j(1)} \mathcal{P}_{j}^{i}\right)+\frac{4}{\bar{\chi}} S_{\perp}^{i(1)}+\partial_{\perp}^{i}\left(2 v_{\|}^{(1)}+h_{l}^{l(1)}+2 \delta_{g}^{(1)}\right)\right] \\
& \times \int_{0}^{\bar{\chi}} \mathrm{d} \tilde{\chi}\left[\frac{\bar{\chi}}{\tilde{\chi}}\left(B_{\perp i}^{(1)}+n^{k} h_{k}^{j(1)} \mathcal{P}_{i j}\right)+(\bar{\chi}-\tilde{\chi}) \tilde{\partial}_{\perp i}\left(A^{(1)}-B_{\|}^{(1)}-\frac{1}{2} h_{\|}^{(1)}\right)\right] \\
& -2\left(A^{(1)}-B_{\|}^{(1)}-\frac{1}{2} h_{\|}^{(1)}-2 I^{(1)}\right) \int_{0}^{\bar{\chi}} \mathrm{d} \tilde{\chi}\left\{\frac{2}{\tilde{\chi}} B_{\|}^{(1)}-\tilde{\partial}_{\perp m} B^{m(1)}-\frac{1}{\tilde{\chi}} h_{i}^{i(1)}+\frac{3}{\tilde{\chi}} h_{\|}^{(1)}-n^{m} \tilde{\partial}_{\perp n} h_{m}^{n(1)}\right. \\
& \left.-\frac{\tilde{\chi}}{\bar{\chi}}\left[2 \tilde{\partial}_{\|}+(\bar{\chi}-\tilde{\chi}) \mathcal{P}^{m n} \tilde{\partial}_{m} \tilde{\partial}_{n}\right]\left(A^{(1)}-B_{\|}^{(1)}-\frac{1}{2} h_{\|}^{(1)}\right)\right\}-\int_{0}^{\bar{\chi}} \mathrm{d} \tilde{\chi}\left\{\frac{1}{\tilde{\chi}} \mathcal{P}_{j}^{i} B_{\|}^{(1)}-\mathcal{P}_{p}^{i} \tilde{\partial}_{\perp j} B^{p(1)}-\frac{1}{\tilde{\chi}} \mathcal{P}_{j}^{p} \mathcal{P}_{q}^{i} h_{p}^{q(1)}+\frac{1}{\tilde{\chi}} \mathcal{P}_{j}^{i} h_{\|}^{(1)}\right. \\
& \left.-n^{p} \mathcal{P}_{q}^{i} \tilde{\partial}_{\perp j} h_{p}^{q(1)}-\frac{\tilde{\chi}}{\bar{\chi}}\left[\mathcal{P}_{j}^{i} \tilde{\partial}_{\|}+(\bar{\chi}-\tilde{\chi}) \mathcal{P}_{j}^{p} \mathcal{P}^{i q} \tilde{\partial}_{q} \tilde{\partial}_{p}\right]\left(A^{(1)}-B_{\|}^{(1)}-\frac{1}{2} h_{\|}^{(1)}\right)\right\} \int_{0}^{\bar{\chi}} \mathrm{d} \tilde{\chi}\left\{\frac{1}{\tilde{\chi}} \mathcal{P}_{i}^{j} B_{\|}^{(1)}-\mathcal{P}_{m}^{j} \tilde{\partial}_{\perp i} B^{m(1)}\right. \\
& \left.+\frac{1}{\tilde{\chi}} \mathcal{P}_{i}^{j} h_{\|}^{(1)}-\frac{1}{\tilde{\chi}} \mathcal{P}_{i}^{n} \mathcal{P}_{m}^{j} h_{n}^{m(1)}-n^{m} \mathcal{P}_{n}^{j} \tilde{\partial}_{\perp i} h_{m}^{n(1)}-\frac{\tilde{\chi}}{\bar{\chi}}\left[\mathcal{P}_{i}^{j} \tilde{\partial}_{\|}+(\bar{\chi}-\tilde{\chi}) \mathcal{P}_{i}^{n} \mathcal{P}^{j m} \tilde{\partial}_{m} \tilde{\partial}_{n}\right]\left(A^{(1)}-B_{\|}^{(1)}-\frac{1}{2} h_{\|}^{(1)}\right)\right\}-2\left(v_{\| o}^{(1)}\right)^{2} \\
& +v_{\| o}^{(1)} h_{\| o}^{(1)}+4 A_{o}^{(1)} v_{\| o}^{(1)}+A_{o}^{(1)} h_{i o}^{i(1)}-A_{o}^{(1)} h_{\| o}^{(1)}+2\left(B_{\perp i o}^{(1)}-v_{\perp i o}^{(1)}+\frac{1}{2} n^{k} h_{k o}^{j(1)} \mathcal{P}_{i j}\right)\left(B_{\perp o}^{i(1)}-v_{\perp o}^{i(1)}+\frac{1}{2} n^{k} h_{k o}^{j(1)} \mathcal{P}_{j}^{i}\right) \\
& -2\left(A_{o}^{(1)}\right)^{2}-v_{\| o}^{(1)} h_{i o}^{i(1)}-\frac{1}{4} \mathcal{P}_{p}^{m} \mathcal{P}_{n}^{k} h_{m o}^{n(1)} h_{k o}^{p(1)}+2\left(2 A_{o}^{(1)}-2 v_{\| o}^{(1)}-\frac{1}{2} h_{i o}^{i(1)}+\frac{1}{2} h_{\| o}^{(1)}\right)\left(A^{(1)}-B_{\|}^{(1)}-\frac{1}{2} h_{\|}^{(1)}-2 I^{(1)}\right) \\
& +2\left(\mathcal{P}_{j}^{i} A_{o}^{(1)}-\mathcal{P}_{j}^{i} v_{\| o}^{(1)}-\frac{1}{2} \mathcal{P}_{j}^{k} \mathcal{P}_{p}^{i} h_{k o}^{p(1)}\right) \int_{0}^{\bar{\chi}} \mathrm{d} \tilde{\chi}\left\{\frac{1}{\tilde{\chi}} \mathcal{P}_{i}^{j} B_{\|}^{(1)}-\mathcal{P}_{m}^{j} \tilde{\partial}_{\perp i} B^{m(1)}-\frac{1}{\tilde{\chi}} \mathcal{P}_{i}^{n} \mathcal{P}_{m}^{j} h_{n}^{m(1)}+\frac{1}{\tilde{\chi}} \mathcal{P}_{i}^{j} h_{\|}^{(1)}\right. \\
& \left.-n^{m} \mathcal{P}_{n}^{j} \tilde{\partial}_{\perp i} h_{m}^{n(1)}-\frac{\tilde{\chi}}{\bar{\chi}}\left[\mathcal{P}_{i}^{j} \tilde{\partial}_{\|}+(\bar{\chi}-\tilde{\chi}) \mathcal{P}_{i}^{n} \mathcal{P}^{j m} \tilde{\partial}_{m} \tilde{\partial}_{n}\right]\left(A^{(1)}-B_{\|}^{(1)}-\frac{1}{2} h_{\|}^{(1)}\right)\right\} \\
& +\left(B_{\perp i o}^{(1)}-v_{\perp i o}^{(1)}+\frac{1}{2} n^{k} h_{k o}^{j(1)} \mathcal{P}_{i j}\right)\left\{-2\left(B_{\perp}^{i(1)}+n^{m} h_{m}^{l(1)} \mathcal{P}_{l}^{i}\right)+4 S_{\perp}^{i(1)}+\bar{\chi} \partial_{\perp}^{i}\left(2 v_{\|}^{(1)}+h_{l}^{l(1)}+2 \delta_{g}^{(1)}\right)\right. \\
& \left.+2 \partial_{\perp i}\left(\frac{1}{\mathcal{H}} \Delta \ln a^{(1)}+T^{(1)}\right)-2 \frac{1}{\bar{\chi}} \int_{0}^{\bar{\chi}} \mathrm{d} \tilde{\chi}\left[\frac{\bar{\chi}}{\tilde{\chi}}\left(B_{\perp}^{i(1)}+n^{k} h_{k}^{j(1)} \mathcal{P}_{j}^{i}\right)+(\bar{\chi}-\tilde{\chi}) \tilde{\partial}_{\perp}^{i}\left(A^{(1)}-B_{\|}^{(1)}-\frac{1}{2} h_{\|}^{(1)}\right)\right]\right\}
\end{aligned}
$$

where for $\Delta \ln a^{(2)} / 2$ see Eq. $\sqrt{165}$, for $\Delta x_{\|}^{(2)}$ see Eq. 132 , for $\partial_{\|} \Delta x_{\|}^{(2)} / 2$ see Eq. 134 and for $\kappa^{(2)}$ see Eq. 136 .

Equation 158 is the main result of this paper - giving the number counts at second order in a general gauge, valid for any dark energy model and also in metric theories of modified gravity.

We can simplify the result by explicitly introducing the weak lensing shear and rotation, defined in Eqs. 82. 83 . 
This leads to

$$
\begin{aligned}
& \Delta_{g}^{(2)}=\delta_{g}^{(2)}+b_{e} \Delta \ln a^{(2)}+\partial_{\|} \Delta x_{\|}^{(2)}+\frac{2}{\bar{\chi}} \Delta x_{\|}^{(2)}-2 \kappa^{(2)}+A^{(2)}+v_{\|}^{(2)}+\frac{1}{2} h_{i}^{i(2)}+\left(\Delta_{g}^{(1)}\right)^{2}-\left(A^{(1)}\right)^{2}+2 A^{(1)} B_{\|}^{(1)} \\
& +A^{(1)} h_{\|}^{(1)}-B_{\|}^{(1)} h_{\|}^{(1)}+v_{\perp i}^{(1)} v_{\perp}^{i(1)}-2 v_{\perp i}^{(1)} B_{\perp}^{i(1)}+B_{\perp i}^{(1)} B_{\perp}^{i(1)}-\frac{1}{2} h_{i}^{k(1)} h_{k}^{i(1)}-\frac{1}{4}\left(h_{\|}^{(1)}\right)^{2}-\left(\delta_{g}^{(1)}\right)^{2}+v_{\|}^{(1)} h_{\|}^{(1)} \\
& -2\left|\gamma^{(1)}\right|^{2}-2\left(\kappa^{(1)}\right)^{2}+\vartheta_{i j}^{(1)} \vartheta^{i j(1)}-\frac{1}{\mathcal{H}^{2}}\left[\frac{\mathrm{d}}{\mathrm{d} \bar{\chi}}\left(A^{(1)}-v_{\|}^{(1)}\right)+\left(A^{(1) \prime}-B_{\|}^{(1) \prime}-\frac{1}{2} h_{\|}^{(1) \prime}\right)\right]^{2} \\
& -\frac{2}{\mathcal{H}}\left(A^{(1)}-B_{\|}^{(1)}-\frac{1}{2} h_{\|}^{(1)}\right)\left[\frac{\mathrm{d}}{\mathrm{d} \bar{\chi}}\left(A^{(1)}-v_{\|}^{(1)}\right)+\left(A^{(1) \prime}-B_{\|}^{(1) \prime}-\frac{1}{2} h_{\|}^{(1) \prime}\right)\right]-\frac{1}{\mathcal{H}} \frac{\mathrm{d}}{\mathrm{d} \bar{\chi}}\left(2 v_{\|}^{(1)}+h_{i}^{i(1)}+2 \delta_{g}^{(1)}\right) \Delta \ln a^{(1)} \\
& -\partial_{\|}\left(2 v_{\|}^{(1)}+h_{i}^{i(1)}+2 \delta_{g}^{(1)}\right) T^{(1)}-\frac{4}{\bar{\chi}^{2} \mathcal{H}} \Delta \ln a^{(1)} T^{(1)}+2 v_{\perp}^{i(1)} \partial_{\perp i} T^{(1)}-\frac{4}{\bar{\chi} \mathcal{H}} \Delta \ln a^{(1)} \kappa^{(1)}-\frac{4}{\bar{\chi}} T^{(1)} \kappa^{(1)} \\
& +\left[-b_{e}+\frac{\mathrm{d} \ln b_{e}}{\mathrm{~d} \ln \bar{a}}-\left(\frac{\mathcal{H}^{\prime}}{\mathcal{H}^{2}}\right)^{2}-\frac{2}{\bar{\chi}^{2} \mathcal{H}^{2}}\right]\left(\Delta \ln a^{(1)}\right)^{2}-\frac{2}{\bar{\chi}^{2}}\left(T^{(1)}\right)^{2}+2 \frac{\mathcal{H}^{\prime}}{\mathcal{H}^{2}}\left(A^{(1)}-B_{\|}^{(1)}-\frac{1}{2} h_{\|}^{(1)}\right) \Delta \ln a^{(1)} \\
& +2 \frac{\mathcal{H}^{\prime}}{\mathcal{H}^{3}}\left[\frac{\mathrm{d}}{\mathrm{d} \bar{\chi}}\left(A^{(1)}-v_{\|}^{(1)}\right)+\left(A^{(1) \prime}-B_{\|}^{(1) \prime}-\frac{1}{2} h_{\|}^{(1) \prime}\right)\right] \Delta \ln a^{(1)}+2\left[-\left(B_{\perp}^{i(1)}+n^{k} h_{k}^{j(1)} \mathcal{P}_{j}^{i}\right)+2 S_{\perp}^{i(1)}\right] \\
& \times \partial_{\perp i}\left(\frac{1}{\mathcal{H}} \Delta \ln a^{(1)}+T^{(1)}\right)-\left[-\frac{2}{\bar{\chi}}\left(B_{\perp}^{i(1)}+n^{k} h_{k}^{j(1)} \mathcal{P}_{j}^{i}\right)+\frac{4}{\bar{\chi}} S_{\perp}^{i(1)}+\partial_{\perp}^{i}\left(2 v_{\|}^{(1)}+h_{l}^{l(1)}+2 \delta_{g}^{(1)}\right)\right] \\
& \times \int_{0}^{\bar{\chi}} \mathrm{d} \tilde{\chi}\left[\frac{\bar{\chi}}{\tilde{\chi}}\left(B_{\perp i}^{(1)}+n^{k} h_{k}^{j(1)} \mathcal{P}_{i j}\right)+(\bar{\chi}-\tilde{\chi}) \tilde{\partial}_{\perp i}\left(A^{(1)}-B_{\|}^{(1)}-\frac{1}{2} h_{\|}^{(1)}\right)\right] \\
& +2\left(B_{\perp i o}^{(1)}-v_{\perp i o}^{(1)}+\frac{1}{2} n^{k} h_{k o}^{j(1)} \mathcal{P}_{i j}\right)\left(B_{\perp o}^{i(1)}-v_{\perp o}^{i(1)}+\frac{1}{2} n^{k} h_{k o}^{j(1)} \mathcal{P}_{j}^{i}\right) \\
& +\left(B_{\perp i o}^{(1)}-v_{\perp i o}^{(1)}+\frac{1}{2} n^{k} h_{k o}^{j(1)} \mathcal{P}_{i j}\right)\left\{-2\left(B_{\perp}^{i(1)}+n^{m} h_{m}^{l(1)} \mathcal{P}_{l}^{i}\right)+4 S_{\perp}^{i(1)}+\bar{\chi} \partial_{\perp}^{i}\left(2 v_{\|}^{(1)}+h_{l}^{l(1)}+2 \delta_{g}^{(1)}\right)\right. \\
& \left.+2 \partial_{\perp i}\left(\frac{1}{\mathcal{H}} \Delta \ln a^{(1)}+T^{(1)}\right)-2 \frac{1}{\bar{\chi}} \int_{0}^{\bar{\chi}} \mathrm{d} \tilde{\chi}\left[\frac{\bar{\chi}}{\tilde{\chi}}\left(B_{\perp}^{i(1)}+n^{k} h_{k}^{j(1)} \mathcal{P}_{j}^{i}\right)+(\bar{\chi}-\tilde{\chi}) \tilde{\partial}_{\perp}^{i}\left(A^{(1)}-B_{\|}^{(1)}-\frac{1}{2} h_{\|}^{(1)}\right)\right]\right\} .
\end{aligned}
$$

\section{Assuming no velocity bias}

Up to now, we have made no assumption about the CDM velocity $u_{m}^{\mu}$ or about the conservation equations. If we define

$$
\mathcal{E}_{m}^{\mu}=\nabla_{\nu} T_{m}^{\mu \nu}, \quad T_{m}^{\mu \nu}=\rho_{m} u_{m}^{\mu} u_{m}^{\nu}
$$

then $\mathcal{E}_{m}^{\mu}=0$ in General Relativity, in the absence of interactions between CDM and dark energy. However, to include interacting CDM and some modified gravity models, we allow for nonzero $\mathcal{E}_{m}^{\mu}$.

We now assume that galaxy velocities follow the matter velocity field (at first and second order), i.e. $u_{m}^{\mu}=u^{\mu}$. Then the expressions for $\mathcal{E}_{m}^{\mu(n)}$ are given in Eqs. (A11)-(A13).

At first order

$$
\partial_{\|} \Delta x_{\|}^{(1)}=A^{(1)}-v_{\|}^{(1)}-\frac{1}{2} h_{\|}^{(1)}-\frac{1}{\mathcal{H}} \partial_{\|} v_{\|}^{(1)}-\frac{1}{2 \mathcal{H}} h_{\|}^{(1) \prime}+\frac{\bar{a}^{2}}{\mathcal{H} \bar{\rho}_{m}}\left(\mathcal{E}_{m}^{\|(1)}-\mathcal{E}_{m}^{0(0)} v_{\|}^{(1)}\right)-\frac{\mathcal{H}^{\prime}}{\mathcal{H}^{2}} \Delta \ln a^{(1)},
$$

where

$$
\begin{aligned}
& \mathcal{E}_{m}^{\|(1)}=n_{i} \mathcal{E}_{m}^{i(1)}=\frac{\bar{\rho}_{m}}{a^{2}}\left(v_{\|}^{(1) \prime}-B_{\|}^{(1) \prime}+\mathcal{H} v_{\|}^{(1)}-\mathcal{H} B_{\|}^{(1)}+\partial_{\|} A^{(1)}\right)+\mathcal{E}_{m}^{0(0)} v_{\|}^{(1)}, \\
& \mathcal{E}_{m}^{0(0)}=\frac{1}{\bar{a}^{2}}\left(\bar{\rho}_{m}^{\prime}+3 \mathcal{H} \bar{\rho}_{m}\right)=\frac{\mathcal{H} \bar{\rho}_{m}}{\bar{a}^{2}} b_{m} .
\end{aligned}
$$

Here $b_{m}=\mathrm{d}\left(a^{3} \bar{\rho}_{m}\right) / \mathrm{d} \ln \bar{a}$. Then

$$
\begin{aligned}
\Delta_{g}^{(1)}= & \delta_{g}^{(1)}+\left(b_{e}-\frac{\mathcal{H}^{\prime}}{\mathcal{H}^{2}}-\frac{2}{\bar{\chi} \mathcal{H}}\right) \Delta \ln a^{(1)}-\frac{1}{\mathcal{H}} \partial_{\|} v_{\|}^{(1)}-\frac{1}{2 \mathcal{H}} h_{\|}^{(1) \prime}+A^{(1)}-\frac{1}{2} h_{\|}^{(1)}+\frac{1}{2} h_{i}^{i(1)}-b_{m} v_{\|}^{(1)} \\
& +\frac{a^{2}}{\mathcal{H} \bar{\rho}_{m}} \mathcal{E}_{m}^{\|(1)}-\frac{2}{\bar{\chi}} T^{(1)}-2 \kappa^{(1)}
\end{aligned}
$$


This relation generalizes for any gauge the results previously obtained in Refs. 1 5 , and can be apply to general dark energy models, including those where dark energy interacts non-gravitationally with dark matter, and to metric theories of modified gravity as an alternative to dark energy.

At second order, using Eqs. A13 we have

$$
\begin{aligned}
\Delta \ln a^{(2)}= & +A_{o}^{(2)}-v_{\| o}^{(2)}-\left(A_{o}^{(1)}\right)^{2}+2 A_{o}^{(1)} B_{\| o}^{(1)}-\left(B_{\| o}^{(1)}\right)^{2}-6 A_{o}^{(1)} v_{\| o}^{(1)}+2 B_{\| o}^{(1)} v_{\| o}^{(1)}+v_{k o}^{(1)} v_{o}^{k(1)} \\
& -n^{i} h_{i j o}^{(1)} v_{o}^{j(1)}+2\left(A_{o}^{(1)}-v_{\| o}^{(1)}\right)\left[-3 A^{(1)}+2 B_{\|}^{(1)}-\frac{1}{\mathcal{H}} \partial_{\|} v_{\|}^{(1)}-\frac{1}{2 \mathcal{H}} h_{\|}^{(1) \prime}-b_{m} v_{\|}^{(1)}\right. \\
& \left.+\bar{\chi} \partial_{\|}\left(2 A^{(1)}-B_{\|}^{(1)}\right)-\bar{\chi}\left(A^{(1) \prime}+\frac{1}{2} h_{\|}^{(1) \prime}\right)+\frac{a^{2}}{\mathcal{H} \bar{\rho}_{m}} \mathcal{E}_{m}^{\|(1)}+4 I^{(1)}\right]+2\left(B_{\perp o}^{i(1)}-v_{\perp o}^{i(1)}+\frac{1}{2} n^{k} h_{k o}^{j(1)} \mathcal{P}_{j}^{i}\right) \\
& +\left\{B_{\perp i}^{(1)}+\bar{\chi} \partial_{\perp i}\left(A^{(1)}+v_{\|}^{(1)}-B_{\|}^{(1)}\right)-\int_{0}^{\bar{\chi}} \mathrm{d} \tilde{\chi}\left[\tilde{\partial}_{\perp i}\left(2 A^{(1)}-B_{\|}^{(1)}\right)-\left(B_{\perp i}^{(1) \prime}+n^{j} h_{j k}^{(1) \prime} \mathcal{P}_{i}^{k}\right)+\frac{1}{\tilde{\chi}} B_{\perp i}^{(1)}\right]\right\} \\
& -A^{(2)}+v_{\|}^{(2)}+7\left(A^{(1)}\right)^{2}+\left(B_{\|}^{(1)}\right)^{2}-6 A^{(1)} B_{\|}^{(1)}-2\left(\frac{1}{2}+b_{m}\right)\left(v_{\|}^{(1)}\right)^{2}+v_{\perp i}^{(1)} v_{\perp}^{i(1)}+v_{\|}^{(1)} h_{\|}^{(1)} \\
& +2 b_{m} A^{(1)} v_{\|}^{(1)}+2 v_{\|}^{(1)} B_{\|}^{(1)}-2 v_{\perp i}^{(1)} B_{\perp}^{i(1)}+\frac{2}{\mathcal{H}}\left(A^{(1)}-v_{\|}^{(1)}\right)\left(\frac{1}{2} h_{\|}^{(1) \prime}+\partial_{\|} v_{\|}^{(1)}-\frac{a^{2}}{\bar{\rho}_{m}} \mathcal{E}_{m}^{\|(1)}\right) \\
& +4\left[-3 A^{(1)}+2 B_{\|}^{(1)}-\frac{1}{\mathcal{H}} \partial_{\|} v_{\|}^{(1)}-\frac{1}{2 \mathcal{H}} h_{\|}^{(1) \prime}-b_{m} v_{\|}^{(1)}+\frac{\bar{a}^{2}}{\mathcal{H} \bar{\rho}_{m}} \mathcal{E}_{m}^{\|(1)}\right] I^{(1)}+4 v_{\perp i}^{(1)} S_{\perp}^{i(1)} \\
& -2 \partial_{\|}\left(A^{(1)}+v_{\|}^{(1)}-B_{\|}^{(1)}\right) T^{(1)}-2\left[\partial_{\|}\left(2 A^{(1)}-B_{\|}^{(1)}\right)-\left(A^{(1) \prime}+\frac{1}{2} h_{\|}^{(1) \prime}\right)\right] \int_{0}^{\bar{\chi}} \mathrm{d} \tilde{\chi}\left[2 A^{(1)}-B_{\|}^{(1)}\right. \\
& \left.+(\bar{\chi}-\tilde{\chi})\left(A^{(1) \prime}-B_{\|}^{(1) \prime}-\frac{1}{2} h_{\|}^{(1) \prime}\right)\right]-2\left[\partial_{\perp i}\left(A^{(1)}+v_{\|}^{(1)}-B_{\|}^{(1)}\right)-\frac{1}{\bar{\chi}}\left(v_{\perp i}^{(1)}-B_{\perp i}^{(1)}\right)\right] \\
& \int_{0}^{\bar{\chi}} \mathrm{d} \tilde{\chi}\left\{\left(B_{\perp}^{i(1)}+n^{k} h_{k}^{j(1)} \mathcal{P}_{j}^{i}\right)+(\bar{\chi}-\tilde{\chi})\left[\tilde{\partial}_{\perp}^{i}\left(A^{(1)}-B_{\|}^{(1)}-\frac{1}{2} h_{\|}^{(1)}\right)+\frac{1}{\tilde{\chi}}\left(B_{\perp}^{i(1)}+n^{k} h_{k j}^{(1)} \mathcal{P}^{i j}\right)\right]\right\}+2 I^{(2)} \\
& +2 \int_{0}^{\bar{\chi}} \mathrm{d} \tilde{\chi}\left\{\left(B_{\|}^{(1)}+h_{\|}^{(1)}-4 I^{(1)}\right)\left(A^{(1) \prime}-B_{\|}^{(1) \prime}-\frac{1}{2} h_{\|}^{(1) \prime}\right)-\left(A^{(1)}-B_{\|}^{(1)}-\frac{1}{2} h_{\|}^{(1)}\right) \frac{\mathrm{d}}{\mathrm{d} \tilde{\chi}}\left(2 A^{(1)}-B_{\|}^{(1)}\right)\right. \\
& \left.+\left[\left(B_{\perp}^{i(1)}+n^{j} h_{j k}^{(1)} \mathcal{P}^{i k}\right)-2 S_{\perp}^{i(1)}\right]\left[\tilde{\partial}_{\perp i}\left(2 A^{(1)}-B_{\|}^{(1)}\right)-\left(B_{\perp i}^{(1) \prime}+n^{j} h_{j k}^{(1) \prime} \mathcal{P}_{i}^{k}\right)+\frac{1}{\tilde{\chi}} B_{\perp i}^{(1)}\right]\right\} \\
& +\Delta \ln a_{\mathrm{post}-\operatorname{Born}}^{(2)}
\end{aligned}
$$




$$
\begin{aligned}
& \Delta x^{0(2)}=+\frac{1}{\mathcal{H}} A_{o}^{(2)}-\frac{1}{\mathcal{H}} v_{\| o}^{(2)}-\left(\frac{\mathcal{H}^{\prime}}{\mathcal{H}^{3}}+\frac{2}{\mathcal{H}}\right)\left(A_{o}^{(1)}\right)^{2}-\frac{1}{\mathcal{H}}\left(B_{\| o}^{(1)}\right)^{2}+\frac{2}{\mathcal{H}} A_{o}^{(1)} B_{\| o}^{(1)}+2\left(\frac{\mathcal{H}^{\prime}}{\mathcal{H}^{3}}-\frac{2}{\mathcal{H}}\right) A_{o}^{(1)} v_{\| o}^{(1)} \\
& +\frac{2}{\mathcal{H}} B_{\| o}^{(1)} v_{\| o}^{(1)}-\frac{\mathcal{H}^{\prime}}{\mathcal{H}^{3}}\left(v_{\| o}^{(1)}\right)^{2}+\frac{1}{\mathcal{H}} v_{\perp i o}^{(1)} v_{\perp o}^{i(1)}-\frac{1}{\mathcal{H}} n^{i} h_{i j o}^{(1)} v_{o}^{j(1)}+2\left(A_{o}^{(1)}-v_{\| o}^{(1)}\right) \\
& \times\left\{\left(\frac{\mathcal{H}^{\prime}}{\mathcal{H}^{3}}-\frac{2}{\mathcal{H}}\right) A^{(1)}-\left[\frac{\mathcal{H}^{\prime}}{\mathcal{H}^{3}}+\frac{1}{\mathcal{H}}\left(1+b_{m}\right)\right] v_{\|}^{(1)}+\frac{2}{\mathcal{H}} B_{\|}^{(1)}-\frac{\bar{\chi}}{\mathcal{H}} A^{(1) \prime}-\frac{1}{\mathcal{H}^{2}}\left(\partial_{\|} v_{\|}^{(1)}-\frac{\bar{a}^{2}}{\bar{\rho}_{m}} \mathcal{E}^{\|(1)}\right)\right. \\
& \left.-\frac{1}{2}\left(\frac{\bar{\chi}}{\mathcal{H}}+\frac{1}{\mathcal{H}^{2}}\right) h_{\|}^{(1) \prime}+\frac{\bar{\chi}}{\mathcal{H}} \partial_{\|}\left(2 A^{(1)}-B_{\|}^{(1)}\right)-2\left(\frac{\mathcal{H}^{\prime}}{\mathcal{H}^{3}}-\frac{1}{\mathcal{H}}\right) I^{(1)}\right\}+2\left(B_{\perp o}^{i(1)}-v_{\perp o}^{i(1)}+\frac{1}{2} n^{k} h_{k o}^{j(1)} \mathcal{P}_{j}^{i}\right) \\
& \times\left\{\frac{1}{\mathcal{H}} B_{\perp i}^{(1)}+\frac{\bar{\chi}}{\mathcal{H}} \partial_{\perp i}\left(A^{(1)}+v_{\|}^{(1)}-B_{\|}^{(1)}\right)-\frac{1}{\mathcal{H}} \int_{0}^{\bar{\chi}} \mathrm{d} \tilde{\chi}\left[\tilde{\partial}_{\perp i}\left(2 A^{(1)}-B_{\|}^{(1)}\right)-\left(B_{\perp i}^{(1) \prime}+n^{j} h_{j k}^{(1) \prime} \mathcal{P}_{i}^{k}\right)\right.\right. \\
& \left.\left.+\frac{1}{\tilde{\chi}} B_{\perp i}^{(1)}\right]\right\}-\frac{1}{\mathcal{H}} A^{(2)}+\frac{1}{\mathcal{H}} v_{\|}^{(2)}+\left(-\frac{\mathcal{H}^{\prime}}{\mathcal{H}^{3}}+\frac{6}{\mathcal{H}}\right)\left(A^{(1)}\right)^{2}+\frac{1}{\mathcal{H}}\left(B_{\|}^{(1)}\right)^{2}-\frac{6}{\mathcal{H}} A^{(1)} B_{\|}^{(1)}+\frac{2}{\mathcal{H}} v_{\|}^{(1)} B_{\|}^{(1)} \\
& -\left[\frac{\mathcal{H}^{\prime}}{\mathcal{H}^{3}}+\frac{2}{\mathcal{H}}\left(1+b_{m}\right)\right]\left(v_{\|}^{(1)}\right)^{2}+\frac{1}{\mathcal{H}} v_{\perp i}^{(1)} v_{\perp}^{i(1)}+\frac{1}{\mathcal{H}} v_{\|}^{(1)} h_{\|}^{(1)}+2\left[\frac{\mathcal{H}^{\prime}}{\mathcal{H}^{3}}+\frac{1}{\mathcal{H}}\left(1+b_{m}\right)\right] A^{(1)} v_{\|}^{(1)}-\frac{2}{\mathcal{H}} v_{\perp i}^{(1)} B_{\perp}^{i(1)} \\
& +\frac{2}{\mathcal{H}^{2}}\left(A^{(1)}-v_{\|}^{(1)}\right)\left(\frac{1}{2} h_{\|}^{(1) \prime}+\partial_{\|} v_{\|}^{(1)}-\frac{\bar{a}^{2}}{\bar{\rho}_{m}} \mathcal{E}_{m}^{\|(1)}\right)+4\left\{\left(\frac{\mathcal{H}^{\prime}}{\mathcal{H}^{3}}-\frac{2}{\mathcal{H}}\right) A^{(1)}-\left[\frac{\mathcal{H}^{\prime}}{\mathcal{H}^{3}}+\frac{1}{\mathcal{H}}\left(1+b_{m}\right)\right] v_{\|}^{(1)}\right. \\
& \left.+\frac{2}{\mathcal{H}} B_{\|}^{(1)}-\frac{1}{\mathcal{H}^{2}}\left(\frac{1}{2} h_{\|}^{(1) \prime}+\partial_{\|} v_{\|}^{(1)}-\frac{\bar{a}^{2}}{\bar{\rho}_{m}} \mathcal{E}_{m}^{\|(1)}\right)-\left(\frac{\mathcal{H}^{\prime}}{\mathcal{H}^{3}}+\frac{1}{\mathcal{H}}\right) I^{(1)}\right\} I^{(1)}+\frac{4}{\mathcal{H}} v_{\perp i}^{(1)} S_{\perp}^{i(1)} \\
& -\frac{2}{\mathcal{H}} \partial_{\|}\left(A^{(1)}+v_{\|}^{(1)}-B_{\|}^{(1)}\right) T^{(1)}-\frac{2}{\mathcal{H}}\left[\partial_{\|}\left(2 A^{(1)}-B_{\|}^{(1)}\right)-\left(A^{(1) \prime}+\frac{1}{2} h_{\|}^{(1) \prime}\right)\right] \\
& \times \int_{0}^{\bar{\chi}} \mathrm{d} \tilde{\chi}\left[2 A^{(1)}-B_{\|}^{(1)}+(\bar{\chi}-\tilde{\chi})\left(A^{(1) \prime}-B_{\|}^{(1) \prime}-\frac{1}{2} h_{\|}^{(1) \prime}\right)\right]-\frac{2}{\mathcal{H}}\left[\partial_{\perp i}\left(A^{(1)}+v_{\|}^{(1)}-B_{\|}^{(1)}\right)\right. \\
& \left.-\frac{1}{\bar{\chi}}\left(v_{\perp i}^{(1)}-B_{\perp i}^{(1)}\right)\right] \int_{0}^{\bar{\chi}} \mathrm{d} \tilde{\chi}\left\{\left(B_{\perp}^{i(1)}+n^{k} h_{k}^{j(1)} \mathcal{P}_{j}^{i}\right)+(\bar{\chi}-\tilde{\chi})\left[\tilde{\partial}_{\perp}^{i}\left(A^{(1)}-B_{\|}^{(1)}-\frac{1}{2} h_{\|}^{(1)}\right)\right.\right. \\
& \left.\left.+\frac{1}{\tilde{\chi}}\left(B_{\perp}^{i(1)}+n^{k} h_{k j}^{(1)} \mathcal{P}^{i j}\right)\right]\right\}+\frac{2}{\mathcal{H}} I^{(2)}+\frac{2}{\mathcal{H}} \int_{0}^{\bar{\chi}} \mathrm{d} \tilde{\chi}\left\{\left(B_{\|}^{(1)}+h_{\|}^{(1)}-4 I^{(1)}\right)\left(A^{(1) \prime}-B_{\|}^{(1) \prime}-\frac{1}{2} h_{\|}^{(1) \prime}\right)\right. \\
& -\left(A^{(1)}-B_{\|}^{(1)}-\frac{1}{2} h_{\|}^{(1)}\right) \frac{\mathrm{d}}{\mathrm{d} \tilde{\chi}}\left(2 A^{(1)}-B_{\|}^{(1)}\right)+\left[\left(B_{\perp}^{i(1)}+n^{j} h_{j k}^{(1)} \mathcal{P}^{i k}\right)-2 S_{\perp}^{i(1)}\right] \\
& \left.\times\left[\tilde{\partial}_{\perp i}\left(2 A^{(1)}-B_{\|}^{(1)}\right)-\left(B_{\perp i}^{(1) \prime}+n^{j} h_{j k}^{(1) \prime} \mathcal{P}_{i}^{k}\right)+\frac{1}{\tilde{\chi}} B_{\perp i}^{(1)}\right]\right\}+\Delta x_{\text {post-Born }}^{0(2)},
\end{aligned}
$$


$\partial_{\|} \Delta x_{\|}^{(2)}=\left(A_{o}^{(1)}\right)^{2}-2 A_{o}^{(1)} B_{\| o}^{(1)}+\left(B_{\| o}^{(1)}\right)^{2}+2 A_{o}^{(1)} v_{\| o}^{(1)}-2 B_{\| o}^{(1)} v_{\| o}^{(1)}-v_{\| o}^{(1)} h_{\| o}^{(1)}-\frac{1}{4}\left(h_{\| o}^{(1)}\right)^{2}-v_{\perp k o}^{(1)} v_{\perp o}^{k(1)}$

$+n^{i} h_{i k o}^{(1)} \mathcal{P}_{j}^{k} v_{o}^{j(1)}-n^{i} h_{i k o}^{(1)} \mathcal{P}_{j}^{k} B_{o}^{j(1)}-\frac{1}{4} n^{i} h_{i j o}^{(1)} \mathcal{P}_{k}^{j} h_{p o}^{k(1)} n^{p}-B_{\perp o}^{i(1)} B_{\perp i o}^{(1)}+2 v_{\perp o}^{i(1)} B_{\perp i o}^{(1)}+2\left(A_{o}^{(1)}-v_{\| o}^{(1)}\right)$

$\times\left\{\left(-\frac{\mathcal{H}^{\prime}}{\mathcal{H}^{3}}+\frac{1}{\mathcal{H}}\right)\left[\frac{\bar{a}^{2}}{\bar{\rho}_{m}} \mathcal{E}_{m}^{\|(1)}-\partial_{\|} v_{\|}^{(1)}-\mathcal{H}\left(b_{m}+1\right) v_{\|}^{(1)}+\mathcal{H} B_{\|}^{(1)}-\frac{1}{2} h_{\|}^{(1) \prime}\right]-\frac{\bar{\chi}}{\mathcal{H}} \frac{\mathrm{d}}{\mathrm{d} \bar{\chi}}\left[\partial_{\|}\left(2 A^{(1)}-B_{\|}^{(1)}\right)\right.\right.$

$\left.-\left(A^{(1) \prime}+\frac{1}{2} h_{\|}^{(1) \prime}\right)\right]+\left(A^{(1)}-B_{\|}^{(1)}-\frac{1}{2} h_{\|}^{(1)}\right)-\bar{\chi} \frac{\mathrm{d}}{\mathrm{d} \bar{\chi}}\left(A^{(1)}-B_{\|}^{(1)}-\frac{1}{2} h_{\|}^{(1)}\right)-\frac{1}{\mathcal{H}^{2}} \frac{\mathrm{d}}{\mathrm{d} \bar{\chi}}\left[\frac{\bar{a}^{2}}{\bar{\rho}_{m}} \mathcal{E}_{m}^{\|(1)}-\partial_{\|} v_{\|}^{(1)}\right.$

$\left.\left.-\mathcal{H}\left(b_{m}+1\right) v_{\|}^{(1)}+\mathcal{H} B_{\|}^{(1)}-\frac{1}{2} h_{\|}^{(1),}\right]\right\}+2\left(B_{\perp o}^{i(1)}-v_{\perp o}^{i(1)}+\frac{1}{2} n^{k} h_{k o}^{j(1)} \mathcal{P}_{j}^{i}\right)\left[-\frac{1}{\mathcal{H}} \frac{\mathrm{d}}{\mathrm{d} \bar{\chi}} B_{\perp i}^{(1)}+\frac{1}{\mathcal{H}} \partial_{\perp i}\left(A^{(1)}-v_{\|}^{(1)}\right)\right.$

$\left.-\frac{1}{\mathcal{H}}\left(B_{\perp i}^{(1) \prime}+n^{j} h_{j k}^{(1) \prime} \mathcal{P}_{i}^{k}\right)-\frac{\bar{\chi}}{\mathcal{H}} \frac{\mathrm{d}}{\mathrm{d} \bar{\chi}} \partial_{\perp i}\left(A^{(1)}+v_{\|}^{(1)}-B_{\|}^{(1)}\right)+\frac{1}{\mathcal{H} \bar{\chi}} B_{\perp i}^{(1)}-4 \delta_{i l} S_{\perp}^{l(1)}\right]+A^{(2)}-B_{\|}^{(2)}-\frac{1}{2} h_{\|}^{(2)}-\frac{1}{2 \mathcal{H}} h_{\|}^{(2) \prime}$

$+2 \frac{\bar{a}^{2}}{\mathcal{H} \bar{\rho}_{m}}\left[\frac{1}{2} \mathcal{E}_{m}^{\|(2)}-\mathcal{E}_{m}^{0(1)} v_{\|}^{(1)}-\mathcal{E}_{m}^{\|(1)}\left(\delta_{m}^{(1)}-A^{(1)}\right)\right]-2 b_{m}\left(\frac{1}{2} v_{\|}^{(2)}-\delta_{m}^{(1)} v_{\|}^{(1)}+2 A^{(1)} v_{\|}^{(1)}\right)-\frac{2}{\mathcal{H}}\left[\frac{1}{2} \partial_{\|} v_{\|}^{(2)}-v_{\|}^{(1)} \partial_{\|} v_{\|}^{(1)}\right.$

$+\mathcal{H}\left(\frac{1}{2} v_{\|}^{(2)}-\frac{1}{2} B_{\|}^{(2)}\right)-\frac{2}{\bar{\chi}}\left(v_{\|}^{(1)}\right)^{2}-v_{\|}^{(1)} \partial_{\perp j} v_{\perp}^{j(1)}+A^{(1)} B_{\|}^{(1) \prime}+A^{(1) \prime} B_{\|}^{(1)}+\mathcal{H} A^{(1)} B_{\|}^{(1)}+v_{\|}^{(1)} h_{\|}^{(1) \prime}+\mathcal{H} B_{\|}^{(1)} h_{\|}^{(1)}$

$+B_{\|}^{(1) \prime} h_{\|}^{(1)}-A^{(1)} \partial_{\|} A^{(1)}-\partial_{\|} A^{(1)} h_{\|}^{(1)}+v_{\perp k}^{(1)} \partial_{\|} B_{\perp}^{k(1)}-v_{\perp}^{j(1)} \partial_{\perp j} B_{\|}^{(1)}+\frac{1}{\bar{\chi}} v_{\perp}^{j(1)} B_{\perp j}^{(1)}+\mathcal{H} B_{k}^{(1)} \mathcal{P}_{j}^{k} h^{i j(1)} n_{i}$

$\left.+v_{k}^{(1)} \mathcal{P}_{j}^{k} h^{i j(1) \prime} n_{i}+B_{k}^{(1) \prime} \mathcal{P}_{j}^{k} h^{i j(1)} n_{i}-\partial_{k} A^{(1)} \mathcal{P}_{j}^{k} h^{i j(1)} n_{i}\right]-2\left(2 A^{(1)}+h_{\|}^{(1)}\right)\left(A^{(1)}-B_{\|}^{(1)}-\frac{1}{2} h_{\|}^{(1)}\right)$

$-\frac{2}{\mathcal{H}} \frac{\mathrm{d}}{\mathrm{d} \bar{\chi}}\left[\frac{7}{2}\left(A^{(1)}\right)^{2}+\frac{1}{2}\left(B_{\|}^{(1)}\right)^{2}-2 A^{(1)} B_{\|}^{(1)}-A^{(1)} v_{\|}^{(1)}+\frac{1}{2}\left(v_{\|}^{(1)}\right)^{2}+\frac{1}{2} v_{\|}^{(1)} h_{\|}^{(1)}+\frac{1}{2} v_{\perp i}^{(1)} v_{\perp}^{i(1)}-v_{\perp i}^{(1)} B_{\perp}^{i(1)}\right]$

$+\frac{2}{\mathcal{H}^{2}}\left(A^{(1)}-v_{\|}^{(1)}\right) \frac{\mathrm{d}}{\mathrm{d} \bar{\chi}}\left[\frac{\bar{a}^{2}}{\bar{\rho}_{m}} \mathcal{E}_{m}^{\|(1)}-\partial_{\|} v_{\|}^{(1)}-\mathcal{H}\left(b_{m}+1\right) v_{\|}^{(1)}+\mathcal{H} B_{\|}^{(1)}-\frac{1}{2} h_{\|}^{(1) \prime}\right]-\frac{2}{\mathcal{H}}\left(3 A^{(1)}+h_{\|}^{(1)}-v_{\|}^{(1)}\right)$

$\times\left(A^{(1) \prime}-B_{\|}^{(1) \prime}-\frac{1}{2} h_{\|}^{(1) \prime}\right)+\frac{2}{\mathcal{H}}\left(2 A^{(1)}-B_{\|}^{(1)}\right)\left[\partial_{\|}\left(2 A^{(1)}-B_{\|}^{(1)}\right)-\left(A^{(1) \prime}+\frac{1}{2} h_{\|}^{(1) \prime}\right)\right]+\left\{2 \frac{\mathcal{H}^{\prime}}{\mathcal{H}^{3}}\left(A^{(1)}-v_{\|}^{(1)}\right)\right.$

$\left.+\frac{2}{\mathcal{H}}\left(A^{(1)}-B_{\|}^{(1)}-\frac{1}{2} h_{\|}^{(1)}\right)+\frac{2}{\mathcal{H}^{2}}\left[\frac{\bar{a}^{2}}{\bar{\rho}_{m}} \mathcal{E}_{m}^{\|(1)}-\partial_{\|} v_{\|}^{(1)}-\mathcal{H}\left(b_{m}+1\right) v_{\|}^{(1)}+\mathcal{H} B_{\|}^{(1)}-\frac{1}{2} h_{\|}^{(1) \prime}\right]\right\}\left[\frac{\bar{a}^{2}}{\bar{\rho}_{m}} \mathcal{E}_{m}^{\|(1)}-\partial_{\|} v_{\|}^{(1)}\right.$

$\left.-\mathcal{H}\left(b_{m}+1\right) v_{\|}^{(1)}+\mathcal{H} B_{\|}^{(1)}-\frac{1}{2} h_{\|}^{(1),}\right]+\frac{2}{\mathcal{H}}\left[\frac{\mathrm{d}}{\mathrm{d} \bar{\chi}}\left(2 A^{(1)}-B_{\|}^{(1)}\right)-\partial_{\|}\left(A^{(1)}+v_{\|}^{(1)}-B_{\|}^{(1)}\right)\right]\left(A^{(1)}-B_{\|}^{(1)}-\frac{1}{2} h_{\|}^{(1)}\right)$

$+2\left(B_{\perp}^{i(1)}+n^{j} h_{j k}^{(1)} \mathcal{P}^{i k}\right)\left[\frac{1}{2}\left(B_{i \perp}^{(1)}+n^{p} h_{p m}^{(1)} \mathcal{P}_{i}^{m}\right)-\frac{1}{\mathcal{H}} \partial_{\perp i}\left(A^{(1)}-v_{\|}^{(1)}\right)+\frac{1}{\mathcal{H}}\left(B_{\perp i}^{(1) \prime}+n^{j} h_{j k}^{(1) \prime} \mathcal{P}_{i}^{k}\right)\right]+\frac{2}{\mathcal{H}} v_{\perp i}^{(1)}$

$\times \partial_{\perp}^{i}\left(A^{(1)}-B_{\|}^{(1)}-\frac{1}{2} h_{\|}^{(1)}\right)+\frac{2}{\mathcal{H}} \frac{\mathrm{d}}{\mathrm{d} \bar{\chi}} \partial_{\|}\left(A^{(1)}+v_{\|}^{(1)}-B_{\|}^{(1)}\right) T^{(1)}+4\left\{\left(-\frac{\mathcal{H}^{\prime}}{\mathcal{H}^{3}}+\frac{1}{\mathcal{H}}\right)\left[\frac{\bar{a}^{2}}{\bar{\rho}_{m}} \mathcal{E}_{m}^{\|(1)}-\partial_{\|} v_{\|}^{(1)}+\mathcal{H} B_{\|}^{(1)}\right.\right.$

$\left.-\mathcal{H}\left(b_{m}+1\right) v_{\|}^{(1)}-\frac{1}{2} h_{\|}^{(1) \prime}\right]-\frac{1}{\mathcal{H}^{2}} \frac{\mathrm{d}}{\mathrm{d} \bar{\chi}}\left[\frac{\bar{a}^{2}}{\bar{\rho}_{m}} \mathcal{E}_{m}^{\|(1)}-\partial_{\|} v_{\|}^{(1)}-\mathcal{H}\left(b_{m}+1\right) v_{\|}^{(1)}+\mathcal{H} B_{\|}^{(1)}-\frac{1}{2} h_{\|}^{(1) \prime}\right]+\left(A^{(1)}-B_{\|}^{(1)}\right.$

$\left.\left.-\frac{1}{2} h_{\|}^{(1)}\right)\right\} I^{(1)}+2\left\{\frac{\mathrm{d}}{\mathrm{d} \bar{\chi}}\left(A^{(1)}-B_{\|}^{(1)}-\frac{1}{2} h_{\|}^{(1)}\right)+\frac{1}{\mathcal{H}} \frac{\mathrm{d}}{\mathrm{d} \bar{\chi}}\left[\partial_{\|}\left(2 A^{(1)}-B_{\|}^{(1)}\right)-\left(A^{(1) \prime}+\frac{1}{2} h_{\|}^{(1) \prime}\right)\right]\right\} \int_{0}^{\bar{\chi}} \mathrm{d} \tilde{\chi}\left[2 A^{(1)}\right.$

$\left.-B_{\|}^{(1)}+(\bar{\chi}-\tilde{\chi})\left(A^{(1) \prime}-B_{\|}^{(1) \prime}-\frac{1}{2} h_{\|}^{(1) \prime}\right)\right]+4\left[\frac{1}{\mathcal{H}} \partial_{\perp i}\left(A^{(1)}-v_{\|}^{(1)}\right)-\frac{1}{\mathcal{H}}\left(B_{\perp i}^{(1) \prime}+n^{j} h_{j k}^{(1) \prime} \mathcal{P}_{i}^{k}\right)+\frac{1}{\mathcal{H} \bar{\chi}} v_{\perp i}^{(1)}\right.$

$\left.-\frac{1}{\mathcal{H}} \frac{\mathrm{d}}{\mathrm{d} \bar{\chi}} v_{\perp i}^{(1)}-2 S_{\perp}^{j(1)} \delta_{i j}\right] S_{\perp}^{i(1)}+\frac{2}{\mathcal{H}} \frac{\mathrm{d}}{\mathrm{d} \bar{\chi}}\left[\partial_{\perp i}\left(A^{(1)}+v_{\|}^{(1)}-B_{\|}^{(1)}\right)-\frac{1}{\bar{\chi}}\left(v_{\perp i}^{(1)}-B_{\perp i}^{(1)}\right)\right] \int_{0}^{\bar{\chi}} \mathrm{d} \tilde{\chi}\left\{\left(B_{\perp}^{i(1)}+n^{k} h_{k}^{j(1)} \mathcal{P}_{j}^{i}\right)\right.$

$\left.+(\bar{\chi}-\tilde{\chi})\left[\tilde{\partial}_{\perp}^{i}\left(A^{(1)}-B_{\|}^{(1)}-\frac{1}{2} h_{\|}^{(1)}\right)+\frac{1}{\tilde{\chi}}\left(B_{\perp}^{i(1)}+n^{k} h_{k j}^{(1)} \mathcal{P}^{i j}\right)\right]\right\}-\frac{\mathcal{H}^{\prime}}{\mathcal{H}^{2}} \Delta \ln a^{(2)}+\left\{-2 \frac{\mathcal{H}^{\prime}}{\mathcal{H}^{2}}\left(A^{(1)}-B_{\|}^{(1)}-\frac{1}{2} h_{\|}^{(1)}\right)\right.$

$\left.-\frac{2}{\mathcal{H}} \frac{\mathrm{d}}{\mathrm{d} \bar{\chi}}\left(A^{(1)}-B_{\|}^{(1)}-\frac{1}{2} h_{\|}^{(1)}\right)-2\left(\frac{\mathcal{H}^{\prime}}{\mathcal{H}^{3}}+\frac{1}{\mathcal{H}}\right)\left[\frac{\bar{a}^{2}}{\bar{\rho}_{m}} \mathcal{E}_{m}^{\|(1)}-\partial_{\|} v_{\|}^{(1)}+\mathcal{H} B_{\|}^{(1)}-\mathcal{H}\left(b_{m}+1\right) v_{\|}^{(1)}-\frac{1}{2} h_{\|}^{(1),}\right]\right\} \Delta \ln a^{(1)}$

$+\left[-\frac{\mathcal{H}^{\prime \prime}}{\mathcal{H}^{3}}+3\left(\frac{\mathcal{H}^{\prime}}{\mathcal{H}^{2}}\right)^{2}+\frac{\mathcal{H}^{\prime}}{\mathcal{H}^{2}}\right]\left(\Delta \ln a^{(1)}\right)^{2}+2 \int_{0}^{\bar{\chi}} \mathrm{d} \tilde{\chi}\left\{\left(A^{(1)}-B_{\|}^{(1)}-\frac{1}{2} h_{\|}^{(1)}\right)\left[2\left(A^{(1) \prime}-B_{\|}^{(1) \prime}-\frac{1}{2} h_{\|}^{(1) \prime}\right)+\frac{\mathrm{d}}{\mathrm{d} \tilde{\chi}}\left(2 A^{(1)}\right.\right.\right.$

$\left.\left.\left.-B_{\|}^{(1)}\right)\right]-2\left(B_{\perp}^{i(1)}+n^{k} h_{k}^{j(1)} \mathcal{P}_{j}^{i}\right)\left[\tilde{\partial}_{\perp i}\left(A^{(1)}-B_{\|}^{(1)}-\frac{1}{2} h_{\|}^{(1)}\right)+\frac{1}{\tilde{\chi}}\left(B_{\perp i}^{(1)}+n^{m} h_{m p}^{(1)} \mathcal{P}_{i}^{p}\right)\right]\right\}+\partial_{\|} \Delta x_{\| \text {post }- \text { Born }}^{(2)}$. 
We can see immediately that $\partial_{\|} \Delta x_{\|}^{(2)}$ can be further expanded. We apply again Eq. A11, in the following terms

$$
\begin{aligned}
& -\frac{1}{\mathcal{H}} \frac{\mathrm{d}}{\mathrm{d} \bar{\chi}} v_{\perp i}^{(1)}=\frac{1}{\mathcal{H}} \frac{\bar{a}^{2}}{\bar{\rho}_{m}} \mathcal{P}_{i j} \mathcal{E}_{m}^{j(1)}-\frac{1}{\mathcal{H}} \partial_{\|} v_{\perp i}^{(1)}-\left(b_{m}+1\right) v_{\perp i}^{(1)}+B_{\perp i}^{(1)}-\frac{1}{\mathcal{H}} \partial_{\perp i} A^{(1)}+\frac{1}{\mathcal{H}} B_{\perp i}^{(1) \prime} \\
& +\frac{2}{\mathcal{H}} \frac{\mathrm{d}}{\mathrm{d} \bar{\chi}} \partial_{\|}\left(A^{(1)}+v_{\|}^{(1)}-B_{\|}^{(1)}\right) T^{(1)}=+\frac{2}{\mathcal{H}}\left[-\frac{\bar{a}^{2}}{\bar{\rho}_{m}} \partial_{\|} \mathcal{E}_{m}^{\|(1)}+\partial_{\|}^{2} v_{\|}^{(1)}+\mathcal{H}\left(b_{m}+1\right) \partial_{\|} v_{\|}^{(1)}-\mathcal{H} \partial_{\|} B_{\|}^{(1)}-\partial_{\|} A^{(1) \prime}\right. \\
& \left.+2 \partial_{\|}^{2} A^{(1)}-\partial_{\|}^{2} B_{\|}^{(1)}\right] T^{(1)} \\
& -\frac{\bar{\chi}}{\mathcal{H}} \frac{\mathrm{d}}{\mathrm{d} \bar{\chi}} \partial_{\perp i}\left(A^{(1)}+v_{\|}^{(1)}-B_{\|}^{(1)}\right)=-\frac{\bar{\chi}}{\mathcal{H}} \partial_{\perp i}\left[-A^{(1) \prime}-\frac{\bar{a}^{2}}{\bar{\rho}_{m}} \mathcal{E}_{m}^{\|(1)}+\partial_{\|} v_{\|}^{(1)}+\mathcal{H}\left(b_{m}+1\right) v_{\|}^{(1)}-\mathcal{H} B_{\|}^{(1)}+2 \partial_{\|} A^{(1)}\right. \\
& \left.-\partial_{\|} B_{\|}^{(1)}\right]+\frac{1}{\mathcal{H}} \partial_{\perp i}\left(A^{(1)}+v_{\|}^{(1)}-B_{\|}^{(1)}\right), \\
& +\frac{2}{\mathcal{H}} \frac{\mathrm{d}}{\mathrm{d} \bar{\chi}}\left[\partial_{\perp i}\left(A^{(1)}+v_{\|}^{(1)}-B_{\|}^{(1)}\right)-\frac{1}{\bar{\chi}}\left(v_{\perp i}^{(1)}-B_{\perp i}^{(1)}\right)\right]=\frac{2}{\mathcal{H}}\left[\partial _ { \perp i } \left(-A^{(1) \prime}-\frac{\bar{a}^{2}}{\bar{\rho}_{m}} \mathcal{E}_{m}^{\|(1)}+\partial_{\|} v_{\|}^{(1)}+\mathcal{H}\left(b_{m}+1\right) v_{\|}^{(1)}\right.\right. \\
& \left.-\mathcal{H} B_{\|}^{(1)}+2 \partial_{\|} A^{(1)}-\partial_{\|} B_{\|}^{(1)}\right)+\frac{1}{\bar{\chi}}\left(-2 \partial_{\perp i} A^{(1)}-\partial_{\perp i} v_{\|}^{(1)}-\partial_{\|} v_{\perp i}^{(1)}+\partial_{\perp i} B_{\|}^{(1)}+\frac{\bar{a}^{2}}{\bar{\rho}_{m}} \mathcal{P}_{i j} \mathcal{E}_{m}^{j(1)}-\mathcal{H}\left(b_{m}+1\right) v_{\perp i}^{(1)}\right. \\
& \left.\left.+\mathcal{H} B_{\perp i}^{(1)}+\partial_{\|} B_{\perp i}^{(1)}\right)+\frac{1}{\bar{\chi}^{2}}\left(v_{\perp i}^{(1)}-B_{\perp i}^{(1)}\right)\right] \\
& \frac{\mathrm{d}}{\mathrm{d} \bar{\chi}}\left[\frac{\bar{a}^{2}}{\bar{\rho}_{m}} \mathcal{E}_{m}^{\|(1)}-\partial_{\|} v_{\|}^{(1)}-\mathcal{H}\left(b_{m}+1\right) v_{\|}^{(1)}+\mathcal{H} B_{\|}^{(1)}-\frac{1}{2} h_{\|}^{(1) \prime}\right]=-\left(\frac{\bar{a}^{2}}{\bar{\rho}_{m}} \mathcal{E}_{m}^{\|(1)}\right)^{\prime}+\frac{\bar{a}^{2} \mathcal{H}}{\bar{\rho}_{m}}\left(b_{m}+1\right) \mathcal{E}_{m}^{\|(1)} \\
& +\mathcal{H}^{2} \frac{\mathrm{d} b_{m}}{\mathrm{~d} \ln a} v_{\|}^{(1)}+b_{m}\left[-\mathcal{H}^{2}\left(b_{m}+1\right) v_{\|}^{(1)}+\mathcal{H} B_{\|}^{(1) \prime}-2 \mathcal{H} \partial_{\|} v_{\|}^{(1)}+\left(\mathcal{H}^{\prime}-\mathcal{H}^{2}\right) v_{\|}^{(1)}+\mathcal{H}^{2} B_{\|}^{(1)}-\mathcal{H} \partial_{\|} A^{(1)}\right] \\
& -2 \mathcal{H} \partial_{\|} v_{\|}^{(1)}-\partial_{\|}^{2} A^{(1)}+\partial_{\|} B_{\|}^{(1) \prime}-\partial_{\|}^{2} v_{\|}^{(1)}+2 \mathcal{H} \partial_{\|} B_{\|}^{(1)}+\left(\mathcal{H}^{\prime}-\mathcal{H}^{2}\right)\left(v_{\|}^{(1)}-B_{\|}^{(1)}\right)-\mathcal{H} \partial_{\|} A^{(1)}-\frac{1}{2} \frac{\mathrm{d}}{\mathrm{d} \bar{\chi}} h_{\|}^{(1) \prime}, \\
& \frac{\mathrm{d}}{\mathrm{d} \bar{\chi}}\left[\frac{7}{2}\left(A^{(1)}\right)^{2}+\frac{1}{2}\left(B_{\|}^{(1)}\right)^{2}-2 A^{(1)} B_{\|}^{(1)}-A^{(1)} v_{\|}^{(1)}+\frac{1}{2}\left(v_{\|}^{(1)}\right)^{2}+\frac{1}{2} v_{\|}^{(1)} h_{\|}^{(1)}+\frac{1}{2} v_{\perp i}^{(1)} v_{\perp}^{i(1)}-v_{\perp i}^{(1)} B_{\perp}^{i(1)}\right] \\
& =6 A^{(1)} \frac{\mathrm{d}}{\mathrm{d} \bar{\chi}} A^{(1)}-A^{(1)} A^{(1) \prime}+B_{\|}^{(1)} \frac{\mathrm{d}}{\mathrm{d} \bar{\chi}} B_{\|}^{(1)}-2 \frac{\mathrm{d}}{\mathrm{d} \bar{\chi}}\left(A^{(1)} B_{\|}^{(1)}\right)+A^{(1)} B_{\|}^{(1) \prime}+v_{\|}^{(1)} A^{(1) \prime}+\frac{1}{2} v_{\|}^{(1)} \frac{\mathrm{d}}{\mathrm{d} \bar{\chi}} h_{\|}^{(1)} \\
& -A^{(1)} \partial_{\|} v_{\|}^{(1)}-\mathcal{H} A^{(1)} v_{\|}^{(1)}+\mathcal{H} A^{(1)} B_{\|}^{(1)}-v_{\|}^{(1)} B_{\|}^{(1) \prime}+v_{\|}^{(1)} \partial_{\|} v_{\|}^{(1)}+\mathcal{H}\left(v_{\|}^{(1)}\right)^{2}-\mathcal{H} v_{\|}^{(1)} B_{\|}^{(1)}+\frac{1}{2} h_{\|}^{(1)} \partial_{\|} A^{(1)}-\frac{1}{2} h_{\|}^{(1)} B_{\|}^{(1) \prime} \\
& +\frac{1}{2} h_{\|}^{(1)} \partial_{\|} v_{\|}^{(1)}+\frac{\mathcal{H}}{2} v_{\|}^{(1)} h_{\|}^{(1)}-\frac{\mathcal{H}}{2} B_{\|}^{(1)} h_{\|}^{(1)}+\left(B_{\perp i}^{(1)}-v_{\perp i}^{(1)}\right)\left(-\partial_{\|} v_{\perp}^{i(1)}+B_{\perp}^{i(1) \prime}-\mathcal{H} v_{\perp}^{i(1)}+\mathcal{H} B_{\perp}^{i(1)}-\partial_{\perp}^{i} A^{(1)}\right) \\
& -\left(A^{(1)}-v_{\|}^{(1)}-\frac{1}{2} h_{\|}^{(1)}\right)\left(\frac{\bar{a}^{2}}{\bar{\rho}_{m}} \mathcal{E}_{m}^{\|(1)}-\mathcal{H} b_{m} v_{\|}^{(1)}\right)+\left(B_{\perp i}^{(1)}-v_{\perp i}^{(1)}\right)\left(\frac{\bar{a}^{2}}{\bar{\rho}_{m}} \mathcal{P}_{j}^{i} \mathcal{E}_{m}^{j(1)}-\mathcal{H} b_{m} v_{\perp}^{i(1)}\right) .
\end{aligned}
$$


Finally, we get

$$
\begin{aligned}
& \Delta_{g}^{(2)}=\delta_{g}^{(2)}+b_{e} \Delta \ln a^{(2)}+\partial_{\|} \Delta x_{\|}^{(2)}+\frac{2}{\bar{\chi}} \Delta x_{\|}^{(2)}-2 \kappa^{(2)}+A^{(2)}+v_{\|}^{(2)}+\frac{1}{2} h_{i}^{i(2)}+\left(\Delta_{g}^{(1)}\right)^{2}-3\left(A^{(1)}\right)^{2}+4 A^{(1)} B_{\|}^{(1)} \\
& +3 A^{(1)} h_{\|}^{(1)}-2 B_{\|}^{(1)} h_{\|}^{(1)}-\left(v_{\|}^{(1)}\right)^{2}-\left(B_{\|}^{(1)}\right)^{2}-\frac{1}{2} h_{i}^{k(1)} h_{k}^{i(1)}-\frac{3}{4}\left(h_{\|}^{(1)}\right)^{2}+2 A^{(1)} v_{\|}^{(1)}-\frac{1}{\mathcal{H}^{2}}\left(\partial_{\|} v_{\|}^{(1)}\right)^{2}+\frac{1}{\mathcal{H}} A^{(1)} h_{\|}^{(1),} \\
& -\frac{1}{4 \mathcal{H}^{2}}\left(h_{\|}^{(1) \prime}\right)^{2}-\frac{1}{\mathcal{H}} v_{\|}^{(1)} h_{\|}^{(1) \prime}-\frac{1}{2 \mathcal{H}} h_{\|}^{(1)} h_{\|}^{(1) \prime}+\frac{2}{\mathcal{H}} A^{(1)} \partial_{\|} v_{\|}^{(1)}-\frac{2}{\mathcal{H}} v_{\|}^{(1)} \partial_{\|} v_{\|}^{(1)}-\frac{1}{\mathcal{H}} h_{\|}^{(1)} \partial_{\|} v_{\|}^{(1)}-\frac{1}{\mathcal{H}^{2}} h_{\|}^{(1) \prime} \partial_{\|} v_{\|}^{(1)} \\
& +B_{\perp i}^{(1)} B_{\perp}^{i(1)}+v_{\perp i}^{(1)} v_{\perp}^{i(1)}-2 v_{\perp i}^{(1)} B_{\perp}^{i(1)}-8\left(I^{(1)}\right)^{2}+8 A^{(1)} I^{(1)}-8 B_{\|}^{(1)} I^{(1)}-4 h_{\|}^{(1)} I^{(1)}-\left(\delta_{g}^{(1)}\right)^{2}+2 v_{\perp}^{i(1)} \partial_{\perp i} T^{(1)} \\
& +\frac{2}{\mathcal{H}}\left(-\partial_{\|} A^{(1)}+B_{\|}^{(1) \prime}-\partial_{\|} v_{\|}^{(1)}-\mathcal{H} v_{\|}^{(1)}+\mathcal{H} B_{\|}^{(1)}\right) \Delta \ln a^{(1)}-\frac{1}{\mathcal{H}} \frac{\mathrm{d}}{\mathrm{d} \bar{\chi}}\left(h_{i}^{i(1)}+2 \delta_{g}^{(1)}\right) \Delta \ln a^{(1)}-\frac{4}{\bar{\chi}^{2} \mathcal{H}} \Delta \ln a^{(1)} T^{(1)} \\
& +2 \frac{\mathcal{H}^{\prime}}{\mathcal{H}^{2}}\left(A^{(1)}-v_{\|}^{(1)}-\frac{1}{2} h_{\|}^{(1)}-\frac{1}{\mathcal{H}} \partial_{\|} v_{\|}^{(1)}-\frac{1}{2 \mathcal{H}} h_{\|}^{(1) \prime}\right) \Delta \ln a^{(1)}-\partial_{\|}\left(2 v_{\|}^{(1)}+h_{i}^{i(1)}+2 \delta_{g}^{(1)}\right) T^{(1)}-\frac{4}{\bar{\chi} \mathcal{H}} \Delta \ln a^{(1)} \kappa^{(1)} \\
& -\frac{4}{\bar{\chi}} T^{(1)} \kappa^{(1)}+\left[-b_{e}+\frac{\mathrm{d} \ln b_{e}}{\mathrm{~d} \ln \bar{a}}-\left(\frac{\mathcal{H}^{\prime}}{\mathcal{H}^{2}}\right)^{2}-\frac{2}{\bar{\chi}^{2} \mathcal{H}^{2}}\right]\left(\Delta \ln a^{(1)}\right)^{2}-\frac{2}{\bar{\chi}^{2}}\left(T^{(1)}\right)^{2}+2\left[-\left(B_{\perp}^{i(1)}+n^{k} h_{k}^{j(1)} \mathcal{P}_{j}^{i}\right)+2 S_{\perp}^{i(1)}\right] \\
& \times \partial_{\perp i}\left(\frac{1}{\mathcal{H}} \Delta \ln a^{(1)}+T^{(1)}\right)-\left[-\frac{2}{\bar{\chi}}\left(B_{\perp}^{i(1)}+n^{k} h_{k}^{j(1)} \mathcal{P}_{j}^{i}\right)+\frac{4}{\bar{\chi}} S_{\perp}^{i(1)}+\partial_{\perp}^{i}\left(2 v_{\|}^{(1)}+h_{l}^{l(1)}+2 \delta_{g}^{(1)}\right)\right] \\
& \times \int_{0}^{\bar{\chi}} \mathrm{d} \tilde{\chi}\left[\frac{\bar{\chi}}{\tilde{\chi}}\left(B_{\perp i}^{(1)}+n^{k} h_{k}^{j(1)} \mathcal{P}_{i j}\right)+(\bar{\chi}-\tilde{\chi}) \tilde{\partial}_{\perp i}\left(A^{(1)}-B_{\|}^{(1)}-\frac{1}{2} h_{\|}^{(1)}\right)\right] \\
& -2\left(A^{(1)}-B_{\|}^{(1)}-\frac{1}{2} h_{\|}^{(1)}-2 I^{(1)}\right) \int_{0}^{\bar{\chi}} \mathrm{d} \tilde{\chi}\left\{\frac{2}{\tilde{\chi}} B_{\|}^{(1)}-\tilde{\partial}_{\perp m} B^{m(1)}-\frac{1}{\tilde{\chi}} h_{i}^{i(1)}+\frac{3}{\tilde{\chi}} h_{\|}^{(1)}-n^{m} \tilde{\partial}_{\perp n} h_{m}^{n(1)}\right. \\
& \left.-\frac{\tilde{\chi}}{\bar{\chi}}\left[2 \tilde{\partial}_{\|}+(\bar{\chi}-\tilde{\chi}) \mathcal{P}^{m n} \tilde{\partial}_{m} \tilde{\partial}_{n}\right]\left(A^{(1)}-B_{\|}^{(1)}-\frac{1}{2} h_{\|}^{(1)}\right)\right\}-\int_{0}^{\bar{\chi}} \mathrm{d} \tilde{\chi}\left\{\frac{1}{\tilde{\chi}} \mathcal{P}_{j}^{i} B_{\|}^{(1)}-\mathcal{P}_{p}^{i} \tilde{\partial}_{\perp j} B^{p(1)}-\frac{1}{\tilde{\chi}} \mathcal{P}_{j}^{p} \mathcal{P}_{q}^{i} h_{p}^{q(1)}\right. \\
& \left.+\frac{1}{\tilde{\chi}} \mathcal{P}_{j}^{i} h_{\|}^{(1)}-n^{p} \mathcal{P}_{q}^{i} \tilde{\partial}_{\perp j} h_{p}^{q(1)}-\frac{\tilde{\chi}}{\bar{\chi}}\left[\mathcal{P}_{j}^{i} \tilde{\partial}_{\|}+(\bar{\chi}-\tilde{\chi}) \mathcal{P}_{j}^{p} \mathcal{P}^{i q} \tilde{\partial}_{q} \tilde{\partial}_{p}\right]\left(A^{(1)}-B_{\|}^{(1)}-\frac{1}{2} h_{\|}^{(1)}\right)\right\} \\
& \int_{0}^{\bar{\chi}} \mathrm{d} \tilde{\chi}\left\{\frac{1}{\tilde{\chi}} \mathcal{P}_{i}^{j} B_{\|}^{(1)}-\mathcal{P}_{m}^{j} \tilde{\partial}_{\perp i} B^{m(1)}-\frac{1}{\tilde{\chi}} \mathcal{P}_{i}^{n} \mathcal{P}_{m}^{j} h_{n}^{m(1)}+\frac{1}{\tilde{\chi}} \mathcal{P}_{i}^{j} h_{\|}^{(1)}-n^{m} \mathcal{P}_{n}^{j} \tilde{\partial}_{\perp i} h_{m}^{n(1)}\right. \\
& \left.-\frac{\tilde{\chi}}{\bar{\chi}}\left[\mathcal{P}_{i}^{j} \tilde{\partial}_{\|}+(\bar{\chi}-\tilde{\chi}) \mathcal{P}_{i}^{n} \mathcal{P}^{j m} \tilde{\partial}_{m} \tilde{\partial}_{n}\right]\left(A^{(1)}-B_{\|}^{(1)}-\frac{1}{2} h_{\|}^{(1)}\right)\right\}-2\left(v_{\| o}^{(1)}\right)^{2}-2\left(A_{o}^{(1)}\right)^{2}-v_{\| o}^{(1)} h_{i o}^{i(1)}+v_{\| o}^{(1)} h_{\| o}^{(1)} \\
& +4 A_{o}^{(1)} v_{\| o}^{(1)}+A_{o}^{(1)} h_{i o}^{i(1)}-A_{o}^{(1)} h_{\| o}^{(1)}+2\left(B_{\perp i o}^{(1)}-v_{\perp i o}^{(1)}+\frac{1}{2} n^{k} h_{k o}^{j(1)} \mathcal{P}_{i j}\right)\left(B_{\perp o}^{i(1)}-v_{\perp o}^{i(1)}+\frac{1}{2} n^{k} h_{k o}^{j(1)} \mathcal{P}_{j}^{i}\right) \\
& -\frac{1}{4} \mathcal{P}_{p}^{m} \mathcal{P}_{n}^{k} h_{m o}^{n(1)} h_{k o}^{p(1)}+2\left(2 A_{o}^{(1)}-2 v_{\| o}^{(1)}-\frac{1}{2} h_{i o}^{i(1)}+\frac{1}{2} h_{\| o}^{(1)}\right)\left(A^{(1)}-B_{\|}^{(1)}-\frac{1}{2} h_{\|}^{(1)}-2 I^{(1)}\right) \\
& +2\left(\mathcal{P}_{j}^{i} A_{o}^{(1)}-\mathcal{P}_{j}^{i} v_{\| o}^{(1)}-\frac{1}{2} \mathcal{P}_{j}^{k} \mathcal{P}_{p}^{i} h_{k o}^{p(1)}\right) \int_{0}^{\bar{\chi}} \mathrm{d} \tilde{\chi}\left\{\frac{1}{\tilde{\chi}} \mathcal{P}_{i}^{j} B_{\|}^{(1)}-\mathcal{P}_{m}^{j} \tilde{\partial}_{\perp i} B^{m(1)}-\frac{1}{\tilde{\chi}} \mathcal{P}_{i}^{n} \mathcal{P}_{m}^{j} h_{n}^{m(1)}+\frac{1}{\tilde{\chi}} \mathcal{P}_{i}^{j} h_{\|}^{(1)}\right. \\
& \left.-n^{m} \mathcal{P}_{n}^{j} \tilde{\partial}_{\perp i} h_{m}^{n(1)}-\frac{\tilde{\chi}}{\bar{\chi}}\left[\mathcal{P}_{i}^{j} \tilde{\partial}_{\|}+(\bar{\chi}-\tilde{\chi}) \mathcal{P}_{i}^{n} \mathcal{P}^{j m} \tilde{\partial}_{m} \tilde{\partial}_{n}\right]\left(A^{(1)}-B_{\|}^{(1)}-\frac{1}{2} h_{\|}^{(1)}\right)\right\} \\
& +\left(B_{\perp i o}^{(1)}-v_{\perp i o}^{(1)}+\frac{1}{2} n^{k} h_{k o}^{j(1)} \mathcal{P}_{i j}\right)\left\{-2\left(B_{\perp}^{i(1)}+n^{m} h_{m}^{l(1)} \mathcal{P}_{l}^{i}\right)+4 S_{\perp}^{i(1)}+\bar{\chi} \partial_{\perp}^{i}\left(2 v_{\|}^{(1)}+h_{l}^{l(1)}+2 \delta_{g}^{(1)}\right)\right. \\
& \left.+2 \partial_{\perp i}\left(\frac{1}{\mathcal{H}} \Delta \ln a^{(1)}+T^{(1)}\right)-2 \frac{1}{\bar{\chi}} \int_{0}^{\bar{\chi}} \mathrm{d} \tilde{\chi}\left[\frac{\bar{\chi}}{\tilde{\chi}}\left(B_{\perp}^{i(1)}+n^{k} h_{k}^{j(1)} \mathcal{P}_{j}^{i}\right)+(\bar{\chi}-\tilde{\chi}) \tilde{\partial}_{\perp}^{i}\left(A^{(1)}-B_{\|}^{(1)}-\frac{1}{2} h_{\|}^{(1)}\right)\right]\right\} \\
& -\left(\frac{\bar{a}^{2}}{\bar{\rho}_{m} \mathcal{H}} \mathcal{E}_{m}^{\|(1)}-b_{m} v_{\|}^{(1)}\right)^{2}-2\left[A^{(1)}-v_{\|}^{(1)}-\frac{1}{2} h_{\|}^{(1)}-\frac{1}{\mathcal{H}} \partial_{\|} v_{\|}^{(1)}-\frac{1}{2 \mathcal{H}} h_{\|}^{(1) \prime}-\left(1+\frac{\mathcal{H}^{\prime}}{\mathcal{H}^{2}}\right) \Delta \ln a^{(1)}\right] \\
& \times\left(\frac{\bar{a}^{2}}{\bar{\rho}_{m} \mathcal{H}} \mathcal{E}_{m}^{\|(1)}-b_{m} v_{\|}^{(1)}\right) .
\end{aligned}
$$

This is the main result in a general gauge with no velocity bias. If we explicitly identify the weak lensing shear and rotation contributions, we arrive at Eq. (A17). 


\section{PERTURBATIONS IN THE POISSON GAUGE}

The Poisson gauge 35] is defined by $\partial^{i} B_{i}{ }^{(n)}=\partial^{j} h_{i j}{ }^{(n)}=0$. In this case, one scalar degree of freedom is eliminated from $g_{0 i}$ and one scalar and two vector degrees of freedom from $g_{i j}$. In addition, here we neglect vector and tensor perturbations at first order. First-order vector perturbations have a decreasing amplitude and are not generated by inflation. The first-order tensor perturbations give a negligible contribution to second-order perturbations. Then the metric is

$$
\mathrm{d} s^{2}=a(\eta)^{2}\left\{-\left(1+2 \Phi^{(1)}+\Phi^{(2)}\right) \mathrm{d} \eta^{2}+2 \omega_{i}^{(2)} \mathrm{d} \eta \mathrm{d} x^{i}+\left[\delta_{i j}\left(1-2 \Psi^{(1)}-\Psi^{(2)}\right)+\frac{1}{2} \hat{h}_{i j}^{(2)}\right] \mathrm{d} x^{i} \mathrm{~d} x^{j}\right\},
$$

where $A^{(n)}=\Phi^{(n)}, B_{i}^{(1)}=0, B^{(2)}=0, \hat{B}_{i}^{(2)}=-2 \omega_{i}^{(2)}, D^{(n)}=-\Psi^{(n)}, F^{(n)}=0, \hat{F}_{j}^{(n)}=0\left(\right.$ i.e. $h_{i j}^{(1)}=-2 \delta_{i j} \Psi^{(1)}$ and $\left.h_{i j}^{(2)}=-2 \delta_{i j} \Psi^{(2)}+\hat{h}_{i j}^{(2)}\right)$.

For the geodesic equation we obtain, at first order,

$$
\frac{\mathrm{d}}{\mathrm{d} \bar{\chi}}\left(\delta \nu^{(1)}-2 \Phi^{(1)}\right)=\Phi^{(1) \prime}+\Psi^{(1) \prime}, \quad \frac{\mathrm{d}}{\mathrm{d} \bar{\chi}}\left(\delta n^{i(1)}-2 \Psi^{(1)} n^{i}\right)=-\partial^{i}\left(\Phi^{(1)}+\Psi^{(1)}\right),
$$

and, at second order,

$$
\begin{aligned}
& \frac{\mathrm{d}}{\mathrm{d} \bar{\chi}}\left(\delta \nu^{(2)}-2 \Phi^{(2)}-2 \omega_{\|}^{(2)}+4 \Phi^{(1)} \delta \nu^{(1)}\right)=\Phi^{(2) \prime}+2 \omega_{\|}^{(2) \prime}+\Psi^{(2) \prime}-\frac{1}{2} \hat{h}_{\|}^{(2) \prime}+4 \delta n^{i(1)} \partial_{i} \Phi^{(1)}+4 \delta n_{\|}^{(1)} \Psi^{(1) \prime} \\
& +2\left[2 \frac{\mathrm{d}}{\mathrm{d} \bar{\chi}} \Phi^{(1) \prime}+\left(\Phi^{(1) \prime \prime}+\Psi^{(1) \prime \prime}\right)\right]\left(\delta x^{0(1)}+\delta x_{\|}^{(1)}\right)+2 \frac{\mathrm{d}}{\mathrm{d} \bar{\chi}}\left[2 \frac{\mathrm{d}}{\mathrm{d} \bar{\chi}} \Phi^{(1)}+\left(\Phi^{(1) \prime}+\Psi^{(1) \prime}\right)\right] \delta x_{\|}^{(1)} \\
& +2\left\{\partial_{\perp i}\left[2 \frac{\mathrm{d}}{\mathrm{d} \bar{\chi}} \Phi^{(1)}+\left(\Phi^{(1) \prime}+\Psi^{(1) \prime}\right)\right]-\frac{2}{\bar{\chi}} \partial_{\perp i} \Phi^{(1)}\right\} \delta x_{\perp}^{i(1)}, \\
& \frac{\mathrm{d}}{\mathrm{d} \bar{\chi}}\left(\delta n^{i(2)}-2 \omega^{i(2)}-2 \Psi^{(2)} n^{i}+\hat{h}_{j}^{i(2)} n^{j}-4 \delta n^{i(1)} \Psi^{(1)}\right)=-\partial^{i}\left(\Phi^{(2)}+\Psi^{(2)}\right)-2 \partial^{i} \omega_{\|}^{(2)}+\frac{2}{\bar{\chi}} \omega_{\perp}^{i(2)}+\frac{1}{2} \partial^{i} \hat{h}_{\|}^{(2)} \\
& -\frac{1}{\bar{\chi}} \mathcal{P}^{i j} \hat{h}_{j k}^{(2)} n^{k}+4 \delta \nu^{(1)}\left(\partial^{i} \Phi^{(1)}+n^{i} \Psi^{(1) \prime}\right)-4 \delta n_{\|}^{(1)} \partial^{i} \Psi^{(1)}+4 \delta n^{j(1)} n^{i} \partial_{j} \Psi^{(1)} \\
& -2\left[\partial^{i}\left(\Phi^{(1)}+\Psi^{(1)}\right)-2 n^{i} \frac{\mathrm{d}}{\mathrm{d} \bar{\chi}} \Psi^{(1)}\right]^{\prime}\left(\delta x^{0(1)}+\delta x_{\|}^{(1)}\right)-2 \frac{\mathrm{d}}{\mathrm{d} \bar{\chi}}\left[\partial^{i}\left(\Phi^{(1)}+\Psi^{(1)}\right)-2 n^{i} \frac{\mathrm{d}}{\mathrm{d} \bar{\chi}} \Psi^{(1)}\right] \delta x_{\|}^{(1)} \\
& -2\left\{\partial_{\perp l}\left[\partial^{i}\left(\Phi^{(1)}+\Psi^{(1)}\right)-2 n^{i} \frac{\mathrm{d}}{\mathrm{d} \bar{\chi}} \Psi^{(1)}\right]+\frac{2}{\bar{\chi}} \mathcal{P}_{l}^{j}\left(\delta_{j}^{i} \frac{\mathrm{d}}{\mathrm{d} \bar{\chi}} \Psi^{(1)}+n^{i} \partial_{j} \Psi^{(1)}\right)\right\} \delta x_{\perp}^{l(1)} .
\end{aligned}
$$

Using the constraints

$$
\begin{aligned}
\delta \nu_{o}^{(1)} & =\Phi_{o}^{(1)}+v_{\| o}^{(1)}, \quad \delta n_{o}^{\hat{a}(1)}=-v_{o}^{\hat{a}(1)}+n^{\hat{a}} \Psi_{o}^{(1)}, \\
\delta \nu_{o}^{(2)} & =\Phi_{o}^{(2)}+v_{\| o}^{(2)}+2 \omega_{\| o}^{(2)}-3\left(\Phi_{o}^{(1)}\right)^{2}-2 v_{\| o}^{(1)} \Phi_{o}^{(1)}-v_{k o}^{(1)} v_{o}^{k(1)}-2 \Psi_{o}^{(1)} v_{\| o}^{(1)} \\
\delta n_{o}^{\hat{a}(2)} & =-v_{o}^{\hat{a}(2)}+n^{\hat{a}} \Psi_{o}^{(2)}-\frac{1}{2} n^{i} \hat{h}_{i o}^{\hat{a}(2)}+v_{o}^{\hat{a}(1)} v_{\| o}^{(1)}+3 n^{\hat{a}}\left(\Psi_{o}^{(1)}\right)^{2},
\end{aligned}
$$

we obtain at first order

$$
\begin{aligned}
& \delta \nu^{(1)}=-\left(\Phi_{o}^{(1)}-v_{\| o}^{(1)}\right)+2 \Phi^{(1)}+\int_{0}^{\bar{\chi}} \mathrm{d} \tilde{\chi}\left(\Phi^{(1) \prime}+\Psi^{(1) \prime}\right)=-\left(\Phi_{o}^{(1)}-v_{\| o}^{(1)}\right)+2 \Phi^{(1)}-2 I^{(1)}, \\
& \delta n^{i(1)}=-v_{o}^{i(1)}-n^{i} \Psi_{o}^{(1)}+2 n^{i} \Psi^{(1)}-\int_{0}^{\bar{\chi}} \mathrm{d} \tilde{\chi} \tilde{\partial}^{i}\left(\Phi^{(1)}+\Psi^{(1)}\right)=n^{i} \delta n_{\|}^{(1)}+\delta n_{\perp}^{i(1)},
\end{aligned}
$$

where

$$
\delta n_{\|}^{(1)}=\Phi_{o}^{(1)}-v_{\| o}^{(1)}-\Phi^{(1)}+\Psi^{(1)}+2 I^{(1)}, \quad \delta n_{\perp}^{i(1)}=-v_{\perp o}^{i(1)}+2 S_{\perp}^{i(1)}
$$




$$
\begin{aligned}
I^{(1)} & =-\frac{1}{2} \int_{0}^{\bar{\chi}} \mathrm{d} \tilde{\chi}\left(\Phi^{(1) \prime}+\Psi^{(1) \prime}\right), \\
S^{i(1)} & =-\frac{1}{2} \int_{0}^{\bar{\chi}} \mathrm{d} \tilde{\chi}\left[\tilde{\partial}^{i}\left(\Phi^{(1)}+\Psi^{(1)}\right)-\frac{2}{\tilde{\chi}} n^{i} \Psi^{(1)}\right], \\
S_{\perp}^{i(1)} & =-\frac{1}{2} \int_{0}^{\bar{\chi}} \mathrm{d} \tilde{\chi} \tilde{\partial}_{\perp}^{i}\left(\Phi^{(1)}+\Psi^{(1)}\right) \\
S_{\|}^{(1)} & =n_{i} S^{i(1)}=\frac{1}{2}\left(\Phi_{o}^{(1)}+\Psi_{\| o}^{(1)}\right)-\frac{1}{2}\left(\Phi^{(1)}+\Psi^{(1)}\right)+I^{(1)}+\int_{0}^{\bar{\chi}} \mathrm{d} \tilde{\chi} \frac{\Psi^{(1)}}{\tilde{\chi}} .
\end{aligned}
$$

Note the following useful relation

$$
\delta n_{\|}^{(1)}+\delta \nu^{(1)}=\Phi^{(1)}+\Psi^{(1)} .
$$

At second order we find

$$
\begin{aligned}
\delta \nu^{(2)}= & -\Phi_{o}^{(2)}+v_{\| o}^{(2)}+\left(\Phi_{o}^{(1)}\right)^{2}+6 \Phi_{o}^{(1)} v_{\| o}^{(1)}-v_{k o}^{(1)} v_{o}^{k(1)}-2 \Psi_{o}^{(1)} v_{\| o}^{(1)}+4\left(\Phi_{o}^{(1)}-v_{\| o}^{(1)}\right)\left(2 \Phi^{(1)}-2 I^{(1)}\right) \\
& -4 v_{\perp o}^{i(1)} \int_{0}^{\bar{\chi}} \mathrm{d} \tilde{\chi}\left(\tilde{\partial}_{\perp i} \Phi^{(1)}\right)+2 \Phi^{(2)}+2 \omega_{\|}^{(2)}-12\left(\Phi^{(1)}\right)^{2}+16 \Phi^{(1)} I^{(1)}-2 I^{(2)} \\
& +4 \int_{0}^{\bar{\chi}} \mathrm{d} \tilde{\chi}\left\{\left(\Phi^{(1)}+\Psi^{(1)}\right) \frac{\mathrm{d}}{\mathrm{d} \tilde{\chi}} \Phi^{(1)}+\left(\Phi^{(1) \prime}+\Psi^{(1) \prime}\right)\left(\Psi^{(1)}+2 I^{(1)}\right)+2 \tilde{\partial}_{\perp i}\left(\Phi^{(1)}\right) S_{\perp}^{i(1)}\right\} \\
& +\delta \nu_{\text {post-Born }}^{(2)},
\end{aligned}
$$

where

$$
\begin{aligned}
& \delta \nu_{\text {post-Born }}^{(2)}=-4 \Phi_{o}^{(1)}\left(3 v_{\| o}^{(1)}-\Psi_{o}^{(1)}\right)-4\left(\Phi_{o}^{(1)}-v_{\| o}^{(1)}\right)\left(\Phi^{(1)}-I^{(1)}\right)-8 v_{\| o}^{(1)} \int_{0}^{\bar{\chi}} \frac{\mathrm{d} \tilde{\chi}}{\tilde{\chi}} \Phi^{(1)}+4 v_{\perp o}^{i(1)} \int_{0}^{\bar{\chi}} \mathrm{d} \tilde{\chi} \tilde{\partial}_{\perp i} \Phi^{(1)} \\
& +4 \Phi^{(1)}\left(\Phi^{(1)}-\Psi^{(1)}-2 I^{(1)}-2 \kappa^{(1)}\right)+2\left[2 \frac{\mathrm{d}}{\mathrm{d} \bar{\chi}} \Phi^{(1)}+\left(\Phi^{(1) \prime}+\Psi^{(1) \prime}\right)\right] \delta x_{\|}^{(1)}+4 \Phi^{(1) \prime}\left(\delta x^{0(1)}+\delta x_{\|}^{(1)}\right) \\
& +4 \partial_{\perp i} \Phi^{(1)} \delta x_{\perp}^{i(1)}+2 \int_{0}^{\bar{\chi}} \mathrm{d} \tilde{\chi}\left\{\left(\Phi^{(1) \prime \prime}+\Psi^{(1) \prime \prime}\right)\left(\delta x^{0(1)}+\delta x_{\|}^{(1)}\right)-2 \Phi^{(1) \prime}\left(\Phi^{(1)}+\Psi^{(1)}\right)\right. \\
& +\left(\Phi^{(1) \prime}+\Psi^{(1) \prime}\right)\left(\Phi^{(1)}-\Psi^{(1)}-2 I^{(1)}\right)-2 \Phi^{(1)}\left[\frac{\mathrm{d}}{\mathrm{d} \tilde{\chi}}\left(\Phi^{(1)}-\Psi^{(1)}\right)+\left(\Phi^{(1) \prime}+\Psi^{(1) \prime}\right)\right] \\
& \left.-4 \tilde{\partial}_{\perp i} \Phi^{(1)} S_{\perp}^{i(1)}-4 \Phi^{(1)} \tilde{\partial}_{\perp j} S_{\perp}^{j(1)}+4\left(\frac{\mathrm{d}}{\mathrm{d} \tilde{\chi}} \Phi^{(1)}-\frac{1}{\tilde{\chi}} \Phi^{(1)}\right) \kappa^{(1)}+\left[\tilde{\partial}_{\perp i}\left(\Phi^{(1) \prime}+\Psi^{(1) \prime}\right)\right] \delta x_{\perp}^{i(1)}\right\}
\end{aligned}
$$

and, splitting $\delta n^{i(2)}=n^{i} \delta n_{\|}^{(2)}+\delta n_{\perp}^{i(2)}$, we obtain

$$
\begin{aligned}
\delta n_{\|}^{(2)} & =\Phi_{o}^{(2)}-v_{\| o}^{(2)}+\left(v_{\| o}^{(1)}\right)^{2}-\left(\Psi_{o}^{(1)}\right)^{2}-4 \Phi_{o}^{(1)} v_{\| o}^{(1)}+4 v_{\| o}^{(1)} \Psi_{o}^{(1)}-4\left(\Phi_{o}^{(1)}-v_{\| o}^{(1)}\right)\left(\Phi^{(1)}-\Psi^{(1)}\right)+8\left(\Phi_{o}^{(1)}-v_{\| o}^{(1)}\right) I^{(1)} \\
& -4 v_{\perp o}^{i(1)} \int_{0}^{\bar{\chi}} \mathrm{d} \tilde{\chi}\left(\partial_{\perp i} \Psi^{(1)}\right)-\Phi^{(2)}+\Psi^{(2)}-\frac{1}{2} \hat{h}_{\|}^{(2)}+4\left(\Psi^{(1)}\right)^{2}+4\left(\Phi^{(1)}\right)^{2}-4 \Phi^{(1)} \Psi^{(1)}-8\left(\Phi^{(1)}-\Psi^{(1)}\right) I^{(1)} \\
& +2 I^{(2)}+4 \int_{0}^{\bar{\chi}} \mathrm{d} \tilde{\chi}\left[\left(\Phi^{(1)}-2 I^{(1)}\right)\left(\Phi^{(1) \prime}+\Psi^{(1) \prime}\right)+2 \tilde{\partial}_{\perp i} \Psi^{(1)} S_{\perp}^{i(1)}\right]+\delta n_{\| \text {post-Born }}^{(2)},
\end{aligned}
$$


where

$$
\begin{aligned}
& \delta n_{\| \text {post-Born }}^{(2)}=2\left(\Phi_{o}^{(1)}-\Psi_{o}^{(1)}\right)\left(3 v_{\| o}^{(1)}-\Psi_{o}^{(1)}\right)-\left(\Phi_{o}^{(1)}-\Psi_{o}^{(1)}\right)^{2}+2\left(\Phi_{o}^{(1)}-v_{\| o}^{(1)}\right)\left(\Phi^{(1)}-\Psi^{(1)}-2 I^{(1)}\right) \\
& +4 v_{\| o}^{(1)} \int_{0}^{\bar{\chi}} \frac{\mathrm{d} \tilde{\chi}}{\tilde{\chi}}\left(\Phi^{(1)}-\Psi^{(1)}-2 v_{\perp o}^{i(1)} \int_{0}^{\bar{\chi}} \mathrm{d} \tilde{\chi}\left[\tilde{\partial}_{\perp i}\left(\Phi^{(1)}-\Psi^{(1)}\right)\right]-2\left[\frac{\mathrm{d}}{\mathrm{d} \bar{\chi}}\left(\Phi^{(1)}-\Psi^{(1)}\right)+\left(\Phi^{(1) \prime}+\Psi^{(1) \prime}\right)\right] \delta x_{\|}^{(1)}\right. \\
& -2\left(\Phi^{(1) \prime}-\Psi^{(1) \prime}\right)\left(\delta x^{0(1)}+\delta x_{\|}^{(1)}\right)-\left(\Phi^{(1)}-\Psi^{(1)}\right)\left(\Phi^{(1)}-\Psi^{(1)}-4 I^{(1)}\right)+4\left(\Phi^{(1)}-\Psi^{(1)}\right) \kappa^{(1)} \\
& -2\left[\partial_{\perp i}\left(\Phi^{(1)}-\Psi^{(1)}\right)\right] \delta x_{\perp}^{i(1)}+2 \int_{0}^{\bar{\chi}} \mathrm{d} \tilde{\chi}\left\{-\left(\Phi^{(1) \prime \prime}+\Psi^{(1) \prime \prime}\right)\left(\delta x^{0(1)}+\delta x_{\|}^{(1)}\right)\right. \\
& +\left(\Phi^{(1) \prime}-\Psi^{(1) \prime}\right)\left(\Phi^{(1)}+\Psi^{(1)}\right)+2\left(\Phi^{(1) \prime}+\Psi^{(1) \prime}\right) I^{(1)}+2 S_{\perp}^{i(1)} \tilde{\partial}_{\perp i}\left(\Phi^{(1)}-\Psi^{(1)}\right) \\
& \left.+2\left(\Phi^{(1)}-\Psi^{(1)}\right) \tilde{\partial}_{\perp j} S_{\perp}^{j(1)}+2\left[-\frac{\mathrm{d}}{\mathrm{d} \tilde{\chi}}\left(\Phi^{(1)}-\Psi^{(1)}\right)+\frac{1}{\tilde{\chi}}\left(\Phi^{(1)}-\Psi^{(1)}\right)\right] \kappa^{(1)}-\tilde{\partial}_{\perp i}\left(\Phi^{(1) \prime}+\Psi^{(1) \prime}\right) \delta x_{\perp}^{i(1)}\right\}
\end{aligned}
$$

and

$$
\begin{aligned}
& \delta n_{\perp}^{i(2)}=-2 \omega_{\perp o}^{i(2)}-v_{\perp o}^{i(2)}+\frac{1}{2} n^{j} \hat{h}_{j k o}^{(2)} \mathcal{P}^{k i}+v_{\| o}^{(1)} v_{\perp o}^{i(1)}+4 v_{\perp o}^{i(1)} \Psi_{o}^{(1)}+8\left(\Phi_{o}^{(1)}-v_{\| o}^{(1)}\right) S_{\perp}^{i(1)}-4 v_{\perp o}^{i(1)} \Psi^{(1)}+2 \omega_{\perp}^{i(2)} \\
& -n^{j} \hat{h}_{j k}^{(2)} \mathcal{P}^{k i}+8 \Psi^{(1)} S_{\perp}^{i(1)}+2 S_{\perp}^{i(2)}+4 \int_{0}^{\bar{\chi}} \mathrm{d} \tilde{\chi}\left\{+2\left(\Phi^{(1)}-I^{(1)}\right) \tilde{\partial}_{\perp}^{i}\left(\Phi^{(1)}+\Psi^{(1)}\right)-\left(\Phi^{(1)}+\Psi^{(1)}\right) \tilde{\partial}_{\perp}^{i} \Psi^{(1)}\right\} \\
& +\delta n_{\perp \text { post-Born }}^{(2)},
\end{aligned}
$$

where

$$
\begin{aligned}
& +\delta n_{\perp \text { post-Born }}^{(2)}=-2 v_{\perp o}^{i(1)}\left(\Phi_{o}^{(1)}+\Psi_{o}^{(1)}\right)-4\left(\Phi_{o}^{(1)}-v_{\| o}^{(1)}\right) S_{\perp}^{i(1)}-2 v_{\perp o}^{i(1)} \int_{0}^{\bar{\chi}} \frac{\mathrm{d} \tilde{\chi}}{\tilde{\chi}}\left(\Phi^{(1)}+\Psi^{(1)}\right) \\
& -2 \partial_{\perp}^{i}\left(\Phi^{(1)}+\Psi^{(1)}\right) \delta x_{\|}^{(1)}-\frac{2}{\bar{\chi}}\left(\Phi^{(1)}+\Psi^{(1)}\right) \delta x_{\perp}^{i(1)}+2 \int_{0}^{\bar{\chi}} \mathrm{d} \tilde{\chi}\left\{-\tilde{\partial}_{\perp}^{i}\left(\Phi^{(1) \prime}+\Psi^{(1) \prime}\right)\left(\delta x^{0(1)}+\delta x_{\|}^{(1)}\right)\right. \\
& -\tilde{\partial}_{\perp}^{i}\left(\Phi^{(1)}+\Psi^{(1)}\right)\left(\Phi^{(1)}-\Psi^{(1)}-2 I^{(1)}\right)+\frac{2}{\tilde{\chi}}\left(\Phi^{(1)}+\Psi^{(1)}\right) S_{\perp}^{i(1)} \\
& \left.-\left[\mathcal{P}^{i m} \tilde{\partial}_{\perp j} \partial_{\perp m}\left(\Phi^{(1)}+\Psi^{(1)}\right)+\frac{1}{\tilde{\chi}} \mathcal{P}_{j}^{i}\left(\Phi^{(1) \prime}+\Psi^{(1) \prime}\right)+\frac{1}{\tilde{\chi}^{2}} \mathcal{P}_{j}^{i}\left(\Phi^{(1)}+\Psi^{(1)}\right)\right] \delta x_{\perp}^{j(1)}\right\} .
\end{aligned}
$$

Here

$$
\begin{aligned}
I^{(2)} & =-\frac{1}{2} \int_{0}^{\bar{\chi}} \mathrm{d} \tilde{\chi}\left(\Phi^{(2) \prime}+2 \omega_{\|}^{(2) \prime}+\Psi^{(2) \prime}-\frac{1}{2} \hat{h}_{\|}^{(2) \prime}\right), \\
S_{\perp}^{i(2)} & =-\frac{1}{2} \int_{0}^{\bar{\chi}} \mathrm{d} \tilde{\chi}\left[\tilde{\partial}_{\perp}^{i}\left(\Phi^{(2)}+2 \omega_{\|}^{(2)}+\Psi^{(2)}-\frac{1}{2} \hat{h}_{\|}^{(2)}\right)+\frac{1}{\tilde{\chi}}\left(-2 \omega_{\perp}^{i(2)}+n^{k} \hat{h}_{k j}^{(2)} \mathcal{P}^{i j}\right)\right] .
\end{aligned}
$$

Combining Eqs. 88 and 105, we obtain

$$
\begin{aligned}
\delta \nu^{(2)}+\delta n_{\|}^{(2)}= & +\left(\Phi_{o}^{(1)}\right)^{2}+2 \Phi_{o}^{(1)} v_{\| o}^{(1)}+2 v_{\| o}^{(1)} \Psi_{o}^{(1)}-\left(\Psi_{o}^{(1)}\right)^{2}-v_{\perp k o}^{(1)} v_{\perp o}^{k(1)}+4\left(\Phi_{o}^{(1)}-v_{\| o}^{(1)}\right)\left(\Phi^{(1)}+\Psi^{(1)}\right) \\
& +8 v_{\perp i o}^{(1)} S_{\perp}^{i(1)}+\Phi^{(2)}+2 \omega_{\|}^{(2)}+\Psi^{(2)}-\frac{1}{2} \hat{h}_{\|}^{(2)}-8\left(\Phi^{(1)}\right)^{2}-4 \Psi^{(1)}\left(\Phi^{(1)}-\Psi^{(1)}\right)+8\left(\Phi^{(1)}+\Psi^{(1)}\right) I^{(1)} \\
& -8 S_{\perp}^{i(1)} S_{\perp}^{j(1)} \delta_{i j}+4 \int_{0}^{\bar{\chi}} \mathrm{d} \tilde{\chi}\left\{\left(\Phi^{(1)}+\Psi^{(1)}\right)\left[\left(\Phi^{(1) \prime}+\Psi^{(1) \prime}\right)+\frac{\mathrm{d}}{\mathrm{d} \tilde{\chi}} \Phi^{(1)}\right]\right\} \\
& +\left(\delta \nu^{(2)}+\delta n_{\|}^{(2)}\right)_{\text {post-Born }}
\end{aligned}
$$


where

$$
\begin{aligned}
& \left(\delta \nu^{(2)}+\delta n_{\|}^{(2)}\right)_{\text {post-Born }}=-2\left(\Phi_{o}^{(1)}+\Psi_{o}^{(1)}\right)\left(3 v_{\| o}^{(1)}-\Psi_{o}^{(1)}\right)-2\left(\Phi_{o}^{(1)}-v_{\| o}^{(1)}\right)\left(\Phi^{(1)}+\Psi^{(1)}\right)-4 v_{\perp o}^{j(1)} S_{\perp}^{j(1)}-4 v_{\| o}^{(1)} \int_{0}^{\bar{\chi}} \frac{\mathrm{d} \tilde{\chi}}{\tilde{\chi}}\left(\Phi^{(1)}\right. \\
& +2 \frac{\mathrm{d}}{\mathrm{d} \bar{\chi}}\left(\Phi^{(1)}+\Psi^{(1)}\right) \delta x_{\|}^{(1)}+2\left(\Phi^{(1) \prime}+\Psi^{(1) \prime}\right)\left(\delta x^{0(1)}+\delta x_{\|}^{(1)}\right)+2\left(\Phi^{(1)}+\Psi^{(1)}\right)\left(\Phi^{(1)}-\Psi^{(1)}-2 I^{(1)}\right) \\
& +2 \partial_{\perp i}\left(\Phi^{(1)}+\Psi^{(1)}\right) \delta x_{\perp}^{i(1)}-4\left(\Phi^{(1)}+\Psi^{(1)}\right) \kappa^{(1)}+2 \int_{0}^{\bar{\chi}} \mathrm{d} \tilde{\chi}\left\{-\left(\Phi^{(1)}+\Psi^{(1)}\right)\left[\frac{\mathrm{d}}{\mathrm{d} \tilde{\chi}}\left(\Phi^{(1)}-\Psi^{(1)}\right)\right.\right. \\
& \left.+2\left(\Phi^{(1) \prime}+\Psi^{(1) \prime}\right)\right]-2 \tilde{\partial}_{\perp j}\left(\Phi^{(1)}+\Psi^{(1)}\right) S_{\perp}^{j(1)}-2\left(\Phi^{(1)}+\Psi^{(1)}\right) \tilde{\partial}_{\perp m} S_{\perp}^{m(1)} \\
& \left.+2\left[\frac{\mathrm{d}}{\mathrm{d} \tilde{\chi}}\left(\Phi^{(1)}+\Psi^{(1)}\right)-\frac{1}{\tilde{\chi}}\left(\Phi^{(1)}+\Psi^{(1)}\right)\right] \kappa^{(1)}\right\}
\end{aligned}
$$

From Eqs 16) and (17), we find

$$
\begin{aligned}
\delta x^{0(1)} & =-\bar{\chi}\left(\Phi_{o}^{(1)}-v_{\| o}^{(1)}\right)+\int_{0}^{\bar{\chi}} \mathrm{d} \tilde{\chi}\left[2 \Phi^{(1)}+(\bar{\chi}-\tilde{\chi})\left(\Phi^{(1) \prime}+\Psi^{(1) \prime}\right)\right] \\
\delta x_{\|}^{(1)} & =\bar{\chi}\left(\Phi_{o}^{(1)}-v_{\| o}^{(1)}\right)-\int_{0}^{\bar{\chi}} \mathrm{d} \tilde{\chi}\left[\left(\Phi^{(1)}-\Psi^{(1)}\right)+(\bar{\chi}-\tilde{\chi})\left(\Phi^{(1) \prime}+\Psi^{(1) \prime}\right)\right], \\
\delta x_{\perp}^{i(1)} & =-\bar{\chi} v_{\perp o}^{i(1)}-\int_{0}^{\bar{\chi}} \mathrm{d} \tilde{\chi}\left[(\bar{\chi}-\tilde{\chi}) \tilde{\partial}_{\perp}^{i}\left(\Phi^{(1)}+\Psi^{(1)}\right)\right],
\end{aligned}
$$

to first order. Using Eq. 103, we note that

$$
T^{(1)}=-\left(\delta x^{0(1)}+\delta x_{\|}^{(1)}\right)=-\int_{0}^{\bar{\chi}} \mathrm{d} \tilde{\chi}\left(\Phi^{(1)}+\Psi^{(1)}\right) .
$$

At second order,

$$
\begin{aligned}
\delta x^{0(2)} & =\bar{\chi}\left[-\Phi_{o}^{(2)}+v_{\| o}^{(2)}+\left(\Phi_{o}^{(1)}\right)^{2}+6 \Phi_{o}^{(1)} v_{\| o}^{(1)}-v_{k o}^{(1)} v_{o}^{k(1)}-2 \Psi_{o}^{(1)} v_{\| o}^{(1)}\right] \\
& +4\left(\Phi_{o}^{(1)}-v_{\| o}^{(1)}\right) \int_{0}^{\bar{\chi}} \mathrm{d} \tilde{\chi}\left[2 \Phi^{(1)}+(\bar{\chi}-\tilde{\chi})\left(\Phi^{(1) \prime}+\Psi^{(1) \prime}\right)\right] \\
& -4 v_{\perp o}^{i(1)} \int_{0}^{\bar{\chi}} \mathrm{d} \tilde{\chi}(\bar{\chi}-\tilde{\chi}) \tilde{\partial}_{\perp i} \Phi^{(1)}+2 \int_{0}^{\bar{\chi}} \mathrm{d} \tilde{\chi}\left[\Phi^{(2)}+\omega_{\|}^{(2)}-6\left(\Phi^{(1)}\right)^{2}+8 \Phi^{(1)} I^{(1)}\right] \\
& +\int_{0}^{\bar{\chi}} \mathrm{d} \tilde{\chi}(\bar{\chi}-\tilde{\chi})\left\{\Phi^{(2) \prime}+2 \omega_{\|}^{(2) \prime}+\Psi^{(2)}-\frac{1}{2} \hat{h}_{\|}^{(2) \prime}+4\left(\Phi^{(1)}+\Psi^{(1)}\right) \frac{\mathrm{d}}{\mathrm{d} \tilde{\chi}} \Phi^{(1)}\right. \\
& \left.+4\left(\Phi^{(1) \prime}+\Psi^{(1) \prime}\right)\left(\Psi^{(1)}+2 I^{(1)}\right)+8 S_{\perp}^{i(1)} \tilde{\partial}_{\perp i} \Phi^{(1)}\right\}+\delta x_{\text {post-Born }}^{0(2)},
\end{aligned}
$$

where

$$
\begin{aligned}
& \delta x_{\text {post-Born }}^{0(2)}=-4 \bar{\chi} \Phi_{o}^{(1)}\left(3 v_{\| o}^{(1)}-\Psi_{o}^{(1)}\right)+4\left(\Phi_{o}^{(1)}-v_{\| o}^{(1)}\right)\left[\bar{\chi} \Phi^{(1)}-\int_{0}^{\bar{\chi}} \mathrm{d} \tilde{\chi}\left(2 \Phi^{(1)}-I^{(1)}\right)\right]-8 v_{\| o}^{(1)} \int_{0}^{\bar{\chi}} \mathrm{d} \tilde{\chi} \frac{(\bar{\chi}-\tilde{\chi})}{\tilde{\chi}} \Phi^{(1)} \\
& +4 v_{\perp o}^{i(1)} \int_{0}^{\bar{\chi}} \mathrm{d} \tilde{\chi}(\bar{\chi}-\tilde{\chi}) \tilde{\partial}_{\perp i} \Phi^{(1)}-4 \Phi^{(1)} \int_{0}^{\bar{\chi}} \mathrm{d} \tilde{\chi}\left(\Phi^{(1)}-\Psi^{(1)}-2 I^{(1)}\right)+2 \int_{0}^{\bar{\chi}} \mathrm{d} \tilde{\chi}\left\{\left(\Phi^{(1) \prime}+\Psi^{(1) \prime}\right) \delta x_{\|}^{(1)}\right. \\
& \left.-2 \Phi^{(1) \prime} T^{(1)}+4 \Phi^{(1)}\left(\Phi^{(1)}-\Psi^{(1)}-2 I^{(1)}-\kappa^{(1)}\right)+2 \tilde{\partial}_{\perp i} \Phi^{(1)} \delta x_{\perp}^{i(1)}\right\}+2 \int_{0}^{\bar{\chi}} \mathrm{d} \tilde{\chi}(\bar{\chi}-\tilde{\chi})\left\{-\left(\Phi^{(1) \prime \prime}+\Psi^{(1) \prime \prime}\right) T^{(1)}\right. \\
& -2 \Phi^{(1) \prime}\left(\Phi^{(1)}+\Psi^{(1)}\right)+\left(\Phi^{(1) \prime}+\Psi^{(1) \prime}\right)\left(\Phi^{(1)}-\Psi^{(1)}-2 I^{(1)}\right)-2 \Phi^{(1)}\left[\frac{\mathrm{d}}{\mathrm{d} \tilde{\chi}}\left(\Phi^{(1)}-\Psi^{(1)}\right)+\left(\Phi^{(1) \prime}+\Psi^{(1) \prime}\right)\right] \\
& \left.-4 \tilde{\partial}_{\perp i} \Phi^{(1)} S_{\perp}^{i(1)}-4 \Phi^{(1)} \tilde{\partial}_{\perp m} S_{\perp}^{m(1)}+4\left(\frac{\mathrm{d}}{\mathrm{d} \tilde{\chi}} \Phi^{(1)}-\frac{1}{\tilde{\chi}} \Phi^{(1)}\right) \kappa^{(1)}+\tilde{\partial}_{\perp i}\left(\Phi^{(1) \prime}+\Psi^{(1) \prime}\right) \delta x_{\perp}^{i(1)}\right\}
\end{aligned}
$$




$$
\begin{aligned}
\delta x_{\|}^{(2)} & =\bar{\chi}\left[\Phi_{o}^{(2)}-v_{\| o}^{(2)}+\left(v_{\| o}^{(1)}\right)^{2}-\left(\Psi_{o}^{(1)}\right)^{2}-4 \Phi_{o}^{(1)} v_{\| o}^{(1)}+4 v_{\| o}^{(1)} \Psi_{o}^{(1)}\right] \\
& -4\left(\Phi_{o}^{(1)}-v_{\| o}^{(1)}\right) \int_{0}^{\bar{\chi}} \mathrm{d} \tilde{\chi}\left[\left(\Phi^{(1)}-\Psi^{(1)}\right)+(\bar{\chi}-\tilde{\chi})\left(\Phi^{(1) \prime}+\Psi^{(1) \prime}\right)\right]-4 v_{\perp o}^{i(1)} \int_{0}^{\bar{\chi}} \mathrm{d} \tilde{\chi}(\bar{\chi}-\tilde{\chi}) \tilde{\partial}_{\perp i} \Psi^{(1)} \\
& +\int_{0}^{\bar{\chi}} \mathrm{d} \tilde{\chi}\left\{-\Phi^{(2)}+\Psi^{(2)}-\frac{1}{2} \hat{h}_{\|}^{(2)}-4 \Psi^{(1)} \Phi^{(1)}+4\left(\Psi^{(1)}\right)^{2}+4\left(\Phi^{(1)}\right)^{2}-8\left(\Phi^{(1)}-\Psi^{(1)}\right) I^{(1)}\right\} \\
& +\int_{0}^{\bar{\chi}} \mathrm{d} \tilde{\chi}(\bar{\chi}-\tilde{\chi})\left\{-\left(\Phi^{(2) \prime}+2 \omega_{\|}^{(2) \prime}+\Psi^{(2) \prime}-\frac{1}{2} \hat{h}_{\|}^{(2) \prime}\right)+4\left(\Phi^{(1)}-2 I^{(1)}\right)\left(\Phi^{(1) \prime}+\Psi^{(1) \prime}\right)+8 S_{\perp}^{i(1)} \tilde{\partial}_{\perp i} \Psi^{(1)}\right\} \\
& +\delta x_{\| \text {post-Born }}^{(2)}
\end{aligned}
$$

where

$$
\begin{aligned}
& \delta x_{\| \text {post-Born }}^{(2)}=2 \bar{\chi}\left(\Phi_{o}^{(1)}-\Psi_{o}^{(1)}\right)\left(3 v_{\| o}^{(1)}-\Psi_{o}^{(1)}\right)-\bar{\chi}\left(\Phi_{o}^{(1)}-\Psi_{o}^{(1)}\right)^{2}+4\left(\Phi_{o}^{(1)}-v_{\| o}^{(1)}\right)\left[-\frac{\bar{\chi}}{2}\left(\Phi^{(1)}-\Psi^{(1)}\right)\right. \\
& \left.+\int_{0}^{\bar{\chi}} \mathrm{d} \tilde{\chi}\left(\Phi^{(1)}-\Psi^{(1)}-I^{(1)}\right)\right]+4 v_{\| o}^{(1)} \int_{0}^{\bar{\chi}} \mathrm{d} \tilde{\chi} \frac{(\bar{\chi}-\tilde{\chi})}{\tilde{\chi}}\left(\Phi^{(1)}-\Psi^{(1)}\right) \\
& -2 v_{\perp o}^{i(1)} \int_{0}^{\bar{\chi}} \mathrm{d} \tilde{\chi}\left[(\bar{\chi}-\tilde{\chi}) \tilde{\partial}_{\perp i}\left(\Phi^{(1)}-\Psi^{(1)}\right)\right]+2\left(\Phi^{(1)}-\Psi^{(1)}\right) \int_{0}^{\bar{\chi}} \mathrm{d} \tilde{\chi}\left(\Phi^{(1)}-\Psi^{(1)}-2 I^{(1)}\right) \\
& +2 \int_{0}^{\bar{\chi}} \mathrm{d} \tilde{\chi}\left\{-\left(\Phi^{(1) \prime}+\Psi^{(1) \prime}\right) \delta x_{\|}^{(1)}-\left(\Phi^{(1)}-\Psi^{(1)}\right)\left[\frac{3}{2}\left(\Phi^{(1)}-\Psi^{(1)}\right)-4 I^{(1)}\right]+\left(\Phi^{(1) \prime}-\Psi^{(1) \prime}\right) T^{(1)}\right. \\
& \left.+2\left(\Phi^{(1)}-\Psi^{(1)}\right) \kappa^{(1)}-\tilde{\partial}_{\perp j}\left(\Phi^{(1)}-\Psi^{(1)}\right) \delta x_{\perp}^{j(1)}\right\}+2 \int_{0}^{\bar{\chi}} \mathrm{d} \tilde{\chi}(\bar{\chi}-\tilde{\chi})\left\{\left(\Phi^{(1) \prime}-\Psi^{(1) \prime}\right)\left(\Phi^{(1)}+\Psi^{(1)}\right)\right. \\
& +\left(\Phi^{(1) \prime \prime}+\Psi^{(1) \prime \prime}\right) T^{(1)}+2\left(\Phi^{(1) \prime}+\Psi^{(1) \prime}\right) I^{(1)}+2 \tilde{\partial}_{\perp i}\left(\Phi^{(1)}-\Psi^{(1)}\right) S_{\perp}^{i(1)} \\
& \left.+2\left(\Phi^{(1)}-\Psi^{(1)}\right) \tilde{\partial}_{\perp m} S_{\perp}^{m(1)}+2\left[-\frac{\mathrm{d}}{\mathrm{d} \tilde{\chi}}\left(\Phi^{(1)}-\Psi^{(1)}\right)+\frac{1}{\tilde{\chi}}\left(\Phi^{(1)}-\Psi^{(1)}\right)\right] \kappa^{(1)}-\left[\tilde{\partial}_{\perp i}\left(\Phi^{(1) \prime}+\Psi^{(1) \prime}\right)\right] \delta x_{\perp}^{i(1)}\right\}
\end{aligned}
$$

and

$$
\begin{aligned}
\delta x_{\perp}^{i(2)} & =\bar{\chi}\left[-2 \omega_{\perp o}^{i(2)}-v_{\perp o}^{i(2)}+\frac{1}{2} n^{j} \hat{h}_{j k o}^{(2)} \mathcal{P}^{k i}+v_{\| o}^{(1)} v_{\perp o}^{i(1)}+4 v_{\perp o}^{i(1)} \Psi_{o}^{(1)}\right] \\
& -4\left(\Phi_{o}^{(1)}-v_{\| o}^{(1)}\right) \int_{0}^{\bar{\chi}} \mathrm{d} \tilde{\chi}(\bar{\chi}-\tilde{\chi}) \tilde{\partial}_{\perp}^{i}\left(\Phi^{(1)}+\Psi^{(1)}\right)-4 v_{\perp o}^{i(1)} \int_{0}^{\bar{\chi}} \mathrm{d} \tilde{\chi} \Psi^{(1)} \\
& +\int_{0}^{\bar{\chi}} \mathrm{d} \tilde{\chi}\left(2 \omega_{\perp}^{i(2)}-n^{j} \hat{h}_{j k}^{(2)} \mathcal{P}^{k i}+8 \Psi^{(1)} S_{\perp}^{i(1)}\right)+\int_{0}^{\bar{\chi}} \mathrm{d} \tilde{\chi}(\bar{\chi}-\tilde{\chi})\left\{-\left[\tilde{\partial}_{\perp}^{i}\left(\Phi^{(2)}+2 \omega_{\|}^{(2)}+\Psi^{(2)}-\frac{1}{2} \hat{h}_{\|}^{(2)}\right)\right.\right. \\
& \left.\left.\left.+\frac{1}{\tilde{\chi}}\left(-2 \omega_{\perp}^{i(2)}+n^{k} \hat{h}_{k j}^{(2)} \mathcal{P}^{i j}\right)\right]+8\left(\Phi^{(1)}-I^{(1)}\right) \tilde{\partial}_{\perp}^{i}\left(\Phi^{(1)}+\Psi^{(1)}\right)-4\left(\Phi^{(1)}+\Psi^{(1)}\right) \tilde{\partial}_{\perp}^{i} \Psi^{(1)}\right]\right\} \\
& +\delta x_{\perp \text { post-Born }}^{i(2)},
\end{aligned}
$$

where

$$
\begin{aligned}
& \delta x_{\perp \text { post-Born }}^{i(2)}=-2 \bar{\chi} v_{\perp o}^{i(1)}\left(\Phi_{o}^{(1)}+\Psi_{o}^{(1)}\right)-4\left(\Phi_{o}^{(1)}-v_{\| o}^{(1)}\right) \int_{0}^{\bar{\chi}} \mathrm{d} \tilde{\chi} S_{\perp}^{i(1)}-2 v_{\perp o}^{i(1)} \int_{0}^{\bar{\chi}} \mathrm{d} \tilde{\chi}(\bar{\chi}-\tilde{\chi}) \frac{1}{\tilde{\chi}}\left(\Phi^{(1)}+\Psi^{(1)}\right) \\
& -2 \int_{0}^{\bar{\chi}} \mathrm{d} \tilde{\chi}\left[\tilde{\partial}_{\perp}^{i}\left(\Phi^{(1)}+\Psi^{(1)}\right) \delta x_{\|}^{(1)}+\frac{1}{\tilde{\chi}}\left(\Phi^{(1)}+\Psi^{(1)}\right) \delta x_{\perp}^{i(1)}\right]+2 \int_{0}^{\bar{\chi}} \mathrm{d} \tilde{\chi}(\bar{\chi}-\tilde{\chi})\left\{-\tilde{\partial}_{\perp}^{i}\left(\Phi^{(1) \prime}+\Psi^{(1) \prime}\right) T^{(1)}\right. \\
& -\tilde{\partial}_{\perp}^{i}\left(\Phi^{(1)}+\Psi^{(1)}\right)\left(\Phi^{(1)}-\Psi^{(1)}-2 I^{(1)}\right)+\frac{2}{\tilde{\chi}}\left(\Phi^{(1)}+\Psi^{(1)}\right) S_{\perp}^{i(1)} \\
& \left.-\left[\mathcal{P}^{i m} \tilde{\partial}_{\perp j} \partial_{\perp m}\left(\Phi^{(1)}+\Psi^{(1)}\right)+\frac{1}{\tilde{\chi}} \mathcal{P}_{j}^{i}\left(\Phi^{(1) \prime}+\Psi^{(1) \prime}\right)+\frac{1}{\tilde{\chi}^{2}} \mathcal{P}_{j}^{i}\left(\Phi^{(1)}+\Psi^{(1)}\right)\right] \delta x_{\perp}^{j(1)}\right\} .
\end{aligned}
$$


Combining Eqs. 113) and 114 we have

$$
\begin{aligned}
\delta x^{0(2)}+\delta x_{\|}^{(2)}= & \bar{\chi}\left[\left(\Phi_{o}^{(1)}\right)^{2}+2 \Phi_{o}^{(1)} v_{\| o}^{(1)}+2 v_{\| o}^{(1)} \Psi_{o}^{(1)}-\left(\Psi_{o}^{(1)}\right)^{2}-v_{\perp k o}^{(1)} v_{\perp o}^{k(1)}\right]-4\left(\Phi_{o}^{(1)}-v_{\| o}^{(1)}\right) T^{(1)} \\
& -4 v_{\perp o}^{i(1)} \int_{0}^{\bar{\chi}} \mathrm{d} \tilde{\chi}(\bar{\chi}-\tilde{\chi})\left[\tilde{\partial}_{\perp}^{i}\left(\Phi^{(1)}+\Psi^{(1)}\right)\right]-T^{(2)}+4 \int_{0}^{\bar{\chi}} \mathrm{d} \tilde{\chi}\left[-2\left(\Phi^{(1)}\right)^{2}\right. \\
& \left.-\Psi^{(1)}\left(\Phi^{(1)}-\Psi^{(1)}\right)+2\left(\Phi^{(1)}+\Psi^{(1)}\right) I^{(1)}-2 S_{\perp}^{i(1)} S_{\perp}^{j(1)} \delta_{i j}\right] \\
& +4 \int_{0}^{\bar{\chi}} \mathrm{d} \tilde{\chi}(\bar{\chi}-\tilde{\chi})\left\{\left(\Phi^{(1)}+\Psi^{(1)}\right)\left[\left(\Phi^{(1) \prime}+\Psi^{(1) \prime}\right)+\frac{\mathrm{d}}{\mathrm{d} \tilde{\chi}} \Phi^{(1)}\right]\right\} \\
& +\left(\delta x^{0(2)}+\delta x_{\|}^{(2)}\right)_{\text {post-Born }},
\end{aligned}
$$

where

$$
\begin{aligned}
& \left(\delta x^{0(2)}+\delta x_{\|}^{(2)}\right)_{\text {post-Born }}=-2 \bar{\chi}\left(\Phi_{o}^{(1)}+\Psi_{o}^{(1)}\right)\left(3 v_{\| o}^{(1)}-\Psi_{o}^{(1)}\right)+4\left(\Phi_{o}^{(1)}-v_{\| o}^{(1)}\right)\left[\frac{\bar{\chi}}{2}\left(\Phi^{(1)}+\Psi^{(1)}\right)+T^{(1)}\right] \\
& -4 v_{\| o}^{(1)} \int_{0}^{\bar{\chi}} \mathrm{d} \tilde{\chi} \frac{(\bar{\chi}-\tilde{\chi})}{\tilde{\chi}}\left(\Phi^{(1)}+\Psi^{(1)}\right)-4 v_{\perp o}^{i(1)} \int_{0}^{\bar{\chi}} \mathrm{d} \tilde{\chi} S_{\perp i}^{(1)}-2\left(\Phi^{(1)}+\Psi^{(1)}\right) \int_{0}^{\bar{\chi}} \mathrm{d} \tilde{\chi}\left(\Phi^{(1)}-\Psi^{(1)}-2 I^{(1)}\right) \\
& +2 \int_{0}^{\bar{\chi}} \mathrm{d} \tilde{\chi}\left\{-\left(\Phi^{(1) \prime}+\Psi^{(1) \prime}\right) T^{(1)}+2\left(\Phi^{(1)}+\Psi^{(1)}\right)\right)\left(\Phi^{(1)}-\Psi^{(1)}-2 I^{(1)}-\kappa^{(1)}\right) \\
& \left.+\left[\tilde{\partial}_{\perp i}\left(\Phi^{(1)}+\Psi^{(1)}\right)\right] \delta x_{\perp}^{i(1)}\right\}+2 \int_{0}^{\bar{\chi}} \mathrm{d} \tilde{\chi}(\bar{\chi}-\tilde{\chi})\left\{-\left(\Phi^{(1)}+\Psi^{(1)}\right)\left[\frac{\mathrm{d}}{\mathrm{d} \tilde{\chi}}\left(\Phi^{(1)}-\Psi^{(1)}\right)+2\left(\Phi^{(1) \prime}+\Psi^{(1)}\right)^{\prime}\right)\right] \\
& \left.-2\left[\tilde{\partial}_{\perp j}\left(\Phi^{(1)}+\Psi^{(1)}\right)\right] S_{\perp}^{i(1)}-2\left(\Phi^{(1)}+\Psi^{(1)}\right) \tilde{\partial}_{\perp m} S_{\perp}^{m(1)}+2\left[\frac{\mathrm{d}}{\mathrm{d} \tilde{\chi}}\left(\Phi^{(1)}+\Psi^{(1)}\right)-\frac{1}{\tilde{\chi}}\left(\Phi^{(1)}+\Psi^{(1)}\right)\right] \kappa^{(1)}\right\} .
\end{aligned}
$$

To obtain all the second order terms we require $\Delta \ln a^{(1)}\left(\right.$ or $\left.\Delta x^{0(1)}\right), \delta \chi^{(1)}, \Delta x^{0(1)}, \Delta x_{\|}^{(1)}, \Delta x_{\perp}^{i(1)}$ and $\Delta_{g}^{(1)}$. From Eqs. 32 and (34) we have

$$
\begin{aligned}
\Delta \ln a^{(1)} & =\left(\Phi_{o}^{(1)}-v_{\| o}^{(1)}\right)-\Phi^{(1)}+v_{\|}^{(1)}+2 I^{(1)}=\left(\Phi_{o}^{(1)}-v_{\| o}^{(1)}\right)-\Phi^{(1)}+v_{\|}^{(1)}-\int_{0}^{\bar{\chi}} \mathrm{d} \tilde{\chi}\left(\Phi^{(1) \prime}+\Psi^{(1) \prime}\right), \\
\delta \chi^{(1)} & =-\left(\bar{\chi}+\frac{1}{\mathcal{H}}\right)\left(\Phi_{o}^{(1)}-v_{\| o}^{(1)}\right)+\frac{1}{\mathcal{H}}\left(\Phi^{(1)}-v_{\|}^{(1)}\right)+\int_{0}^{\bar{\chi}} \mathrm{d} \tilde{\chi}\left[2 \Phi^{(1)}+(\bar{\chi}-\tilde{\chi})\left(\Phi^{(1) \prime}+\Psi^{(1) \prime}\right)\right]-\frac{2}{\mathcal{H}} I^{(1)} .
\end{aligned}
$$

Then, from Eq. (36)

$$
\Delta x^{0(1)}=\frac{1}{\mathcal{H}}\left[\left(\Phi_{o}^{(1)}-v_{\| o}^{(1)}\right)-\Phi^{(1)}+v_{\|}^{(1)}+2 I^{(1)}\right]=\frac{1}{\mathcal{H}}\left[\left(\Phi_{o}^{(1)}-v_{\| o}^{(1)}\right)-\Phi^{(1)}+v_{\|}^{(1)}-\int_{0}^{\bar{\chi}} \mathrm{d} \tilde{\chi}\left(\Phi^{(1) \prime}+\Psi^{(1) \prime}\right)\right]
$$

and from Eqs. 69 and 111

$$
\begin{aligned}
\Delta x_{\|}^{(1)} & =-T^{(1)}-\frac{1}{\mathcal{H}}\left[\left(\Phi_{o}^{(1)}-v_{\| o}^{(1)}\right)-\Phi^{(1)}+v_{\|}^{(1)}+2 I^{(1)}\right] \\
& =\int_{0}^{\bar{\chi}} \mathrm{d} \tilde{\chi}\left(\Phi^{(1)}+\Psi^{(1)}\right)-\frac{1}{\mathcal{H}}\left[\left(\Phi_{o}^{(1)}-v_{\| o}^{(1)}\right)-\Phi^{(1)}+v_{\|}^{(1)}-\int_{0}^{\bar{\chi}} \mathrm{d} \tilde{\chi}\left(\Phi^{(1) \prime}+\Psi^{(1) \prime}\right)\right] .
\end{aligned}
$$

Using Eq. 270, we have

$$
\Delta x_{\perp}^{i(1)}=-\bar{\chi} v_{\perp o}^{i(1)}-\int_{0}^{\bar{\chi}} \mathrm{d} \tilde{\chi}(\bar{\chi}-\tilde{\chi}) \tilde{\partial}_{\perp}^{i}\left(\Phi^{(1)}+\Psi^{(1)}\right)
$$

In Eq. (119) there is an ISW contribution and in Eq. 120 we have both time-delay and ISW contributions. 
Now we can obtain $\Delta_{g}^{(1)}$. Using Eq. $\sqrt{79}$ for $\Delta x_{\|}^{(1)}$, we find

$$
\begin{aligned}
\partial_{\|} \Delta x_{\|}^{(1)} & =\left(\Phi^{(1)}+\Psi^{(1)}\right)-\frac{\mathcal{H}^{\prime}}{\mathcal{H}^{2}} \Delta \ln a^{(1)}-\frac{1}{\mathcal{H}}\left(\frac{\mathrm{d} \Delta \ln a}{\mathrm{~d} \bar{\chi}}\right)^{(1)} \\
& =\left(\Phi^{(1)}+\Psi^{(1)}\right)+\frac{1}{\mathcal{H}}\left[\frac{\mathrm{d}}{\mathrm{d} \bar{\chi}}\left(\Phi^{(1)}-v_{\|}^{(1)}\right)+\left(\Phi^{(1) \prime}+\Psi^{(1) \prime}\right)\right]-\frac{\mathcal{H}^{\prime}}{\mathcal{H}^{2}} \Delta \ln a^{(1)} .
\end{aligned}
$$

With Eqs. 117), 120 and (122, Eq. (76) becomes

$$
\begin{aligned}
\Delta_{g}^{(1)}= & \delta_{g}^{(1)}+\left(b_{e}-\frac{\mathcal{H}^{\prime}}{\mathcal{H}^{2}}-\frac{2}{\bar{\chi} \mathcal{H}}\right) \Delta \ln a^{(1)}+\frac{1}{\mathcal{H}}\left[\frac{\mathrm{d}}{\mathrm{d} \bar{\chi}}\left(\Phi^{(1)}-v_{\|}^{(1)}\right)+\left(\Phi^{(1) \prime}+\Psi^{(1) \prime}\right)\right]-\frac{2}{\bar{\chi}} T^{(1)}-2 \kappa^{(1)} \\
& +\Phi^{(1)}+v_{\|}^{(1)}-2 \Psi^{(1)}
\end{aligned}
$$

in agreement with $[3,4$. At first order, the coordinate convergence lensing term defined in Eq. 80 yields

$$
\kappa^{(1)}=-\frac{1}{2} \partial_{\perp i} \Delta x_{\perp}^{i(1)}=\frac{1}{2} \int_{0}^{\bar{\chi}} \mathrm{d} \tilde{\chi}(\bar{\chi}-\tilde{\chi}) \frac{\tilde{\chi}}{\bar{\chi}} \tilde{\nabla}_{\perp}^{2}\left(\Phi^{(1)}+\Psi^{(1)}\right)-v_{\| o}^{(1)} .
$$

At this point, we can finally compute $\Delta \ln a^{(2)}, \Delta x^{0(2)}, \Delta x_{\|}^{(2)}$ and $\Delta x_{\perp}^{i(2)}$. From Eq. (33) we find

$$
\begin{aligned}
\Delta \ln a^{(2)}= & \Phi_{o}^{(2)}-v_{\| o}^{(2)}-\left(\Phi_{o}^{(1)}\right)^{2}-6 \Phi_{o}^{(1)} v_{\| o}^{(1)}+v_{k o}^{(1)} v_{o}^{k(1)}+2 \Psi_{o}^{(1)} v_{\| o}^{(1)}+2\left(\Phi_{o}^{(1)}-v_{\| o}^{(1)}\right)\left[-3 \Phi^{(1)}+v_{\|}^{(1)}\right. \\
& \left.+\left(2 \bar{\chi}+\frac{1}{\mathcal{H}}\right) \frac{\mathrm{d}}{\mathrm{d} \bar{\chi}} \Phi^{(1)}-\frac{1}{\mathcal{H}} \frac{\mathrm{d}}{\mathrm{d} \bar{\chi}} v_{\|}^{(1)}+\left(\bar{\chi}+\frac{1}{\mathcal{H}}\right)\left(\Phi^{(1) \prime}+\Psi^{(1) \prime}\right)+4 I^{(1)}\right] \\
& -2 v_{\perp o}^{i(1)}\left[\bar{\chi} \partial_{\perp i}\left(\Phi^{(1)}+v_{\|}^{(1)}\right)-2 \int_{0}^{\bar{\chi}} \mathrm{d} \tilde{\chi} \tilde{\partial}_{\perp i} \Phi^{(1)}\right]-\Phi^{(2)}+v_{\|}^{(2)}+7\left(\Phi^{(1)}\right)^{2}+v_{i}^{(1)} v^{i(1)} \\
& -2 v_{\|}^{(1)}\left(\Psi^{(1)}+\Phi^{(1)}\right)-\frac{2}{\mathcal{H}}\left(\Phi^{(1)}-v_{\|}^{(1)}\right)\left[\frac{\mathrm{d}}{\mathrm{d} \bar{\chi}}\left(\Phi^{(1)}-v_{\|}^{(1)}\right)+\left(\Phi^{(1) \prime}+\Psi^{(1) \prime}\right)\right] \\
& -4\left[3 \Phi^{(1)}-v_{\|}^{(1)}-\frac{1}{\mathcal{H}} \frac{\mathrm{d}}{\mathrm{d} \bar{\chi}}\left(\Phi^{(1)}-v_{\|}^{(1)}\right)-\frac{1}{\mathcal{H}}\left(\Phi^{(1) \prime}+\Psi^{(1) \prime}\right)\right] I^{(1)}+4 v_{\perp i}^{(1)} S_{\perp}^{i(1)} \\
& -2 \partial_{\|}\left(\Phi^{(1)}+v_{\|}^{(1)}\right) T^{(1)}-2\left[2 \frac{\mathrm{d}}{\mathrm{d} \bar{\chi}} \Phi^{(1)}+\left(\Phi^{(1) \prime}+\Psi^{(1) \prime}\right)\right] \int_{0}^{\bar{\chi}} \mathrm{d} \tilde{\chi}\left[2 \Phi^{(1)}+(\bar{\chi}-\tilde{\chi})\left(\Phi^{(1) \prime}+\Psi^{(1) \prime}\right)\right] \\
& -2\left[\partial_{\perp i}\left(\Phi^{(1)}+v_{\|}^{(1)}\right)-\frac{1}{\bar{\chi}} v_{\perp i}^{(1)}\right] \int_{0}^{\bar{\chi}} \mathrm{d} \tilde{\chi}\left[\left(\bar{\chi}-\tilde{\chi}_{\perp} \tilde{\partial}_{\perp}^{i}\left(\Phi^{(1)}+\Psi^{(1)}\right)\right]+2 I^{(2)}\right. \\
& -4 \int_{0}^{\bar{\chi}} \mathrm{d} \tilde{\chi}\left\{\left(\Psi^{(1)}+2 I^{(1)}\right)\left(\Phi^{(1) \prime}+\Psi^{(1) \prime}\right)+\left(\Phi^{(1)}+\Psi^{(1)}\right) \frac{\mathrm{d}}{\mathrm{d} \tilde{\chi}} \Phi^{(1)}+2 S_{\perp}^{i(1)} \tilde{\partial}_{\perp i} \Phi^{(1)}\right\} \\
+ & \left.\Delta \ln a_{\mathrm{post}-\operatorname{Born}}^{(2)}\right\}
\end{aligned}
$$

where

$$
\begin{aligned}
& \Delta \ln a_{\text {post-Born }}^{(2)}=4 \Phi_{o}^{(1)}\left(3 v_{\| o}^{(1)}-\Psi_{o}^{(1)}\right)+2\left(\Phi_{o}^{(1)}-v_{\| o}^{(1)}\right)\left\{2 \Phi^{(1)}-2 I^{(1)}-\bar{\chi}\left[2 \frac{\mathrm{d}}{\mathrm{d} \bar{\chi}} \Phi^{(1)}+\left(\Phi^{(1) \prime}+\Psi^{(1) \prime}\right)\right]\right\} \\
& +8 v_{\| o}^{(1)} \int_{0}^{\bar{\chi}} \frac{\mathrm{d} \tilde{\chi}}{\tilde{\chi}} \Phi^{(1)}-4 v_{\perp o}^{i(1)}\left(-\bar{\chi} \partial_{\perp i} \Phi^{(1)}+\int_{0}^{\bar{\chi}} \mathrm{d} \tilde{\chi}_{\perp i} \tilde{\partial}^{(1)}\right)-4 \Phi^{(1)}\left(\Phi^{(1)}-\Psi^{(1)}-2 I^{(1)}-2 \kappa^{(1)}\right)+4 \Phi^{(1) \prime} T^{(1)} \\
& +2\left[2 \frac{\mathrm{d}}{\mathrm{d} \bar{\chi}} \Phi^{(1)}+\left(\Phi^{(1) \prime}+\Psi^{(1) \prime}\right)\right] \int_{0}^{\bar{\chi}} \mathrm{d} \tilde{\chi}\left(\Phi^{(1)}-\Psi^{(1)}-2 I^{(1)}\right)-8 \partial_{\perp i} \Phi^{(1)} \int_{0}^{\bar{\chi}} \mathrm{d} \tilde{\chi} S_{\perp}^{i(1)} \\
& +2 \int_{0}^{\bar{\chi}} \mathrm{d} \tilde{\chi}\left\{+\left(\Phi^{(1) \prime \prime}+\Psi^{(1) \prime \prime}\right) T^{(1)}+2 \Phi^{(1) \prime}\left(\Phi^{(1)}+\Psi^{(1)}\right)-\left(\Phi^{(1) \prime}+\Psi^{(1) \prime}\right)\left(\Phi^{(1)}-\Psi^{(1)}-2 I^{(1)}\right)\right. \\
& +2 \Phi^{(1)}\left[\frac{\mathrm{d}}{\mathrm{d} \tilde{\chi}}\left(\Phi^{(1)}-\Psi^{(1)}\right)+\left(\Phi^{(1) \prime}+\Psi^{(1) \prime}\right)\right]+4 S_{\perp}^{i(1)} \tilde{\partial}_{\perp i} \Phi^{(1)}+4 \Phi^{(1)} \tilde{\partial}_{\perp j} S_{\perp}^{j(1)}-4\left(\frac{\mathrm{d}}{\mathrm{d} \tilde{\chi}} \Phi^{(1)}-\frac{1}{\tilde{\chi}} \Phi^{(1)}\right) \kappa^{(1)} \\
& \left.-\left[\tilde{\partial}_{\perp i}\left(\Phi^{(1) \prime}+\Psi^{(1) \prime}\right)\right] \delta x_{\perp}^{i(1)}\right\} .
\end{aligned}
$$


Using Eqs (117) and 165, Eq. (37) yields

$$
\begin{aligned}
\Delta x^{0(2)}= & +\frac{1}{\mathcal{H}} \Phi_{o}^{(2)}-\frac{1}{\mathcal{H}} v_{\| o}^{(2)}-\left(\frac{\mathcal{H}^{\prime}}{\mathcal{H}^{3}}+\frac{2}{\mathcal{H}}\right)\left(\Phi_{o}^{(1)}\right)^{2}+2\left(\frac{\mathcal{H}^{\prime}}{\mathcal{H}^{3}}-\frac{2}{\mathcal{H}}\right) \Phi_{o}^{(1)} v_{\| o}^{(1)}-\frac{\mathcal{H}^{\prime}}{\mathcal{H}^{3}}\left(v_{\| o}^{(1)}\right)^{2}+\frac{2}{\mathcal{H}} \Psi_{o}^{(1)} v_{\| o}^{(1)} \\
& +\frac{1}{\mathcal{H}} v_{\perp i o}^{(1)} v_{\perp o}^{i(1)}+2\left(\Phi_{o}^{(1)}-v_{\| o}^{(1)}\right)\left\{\left(\frac{\mathcal{H}^{\prime}}{\mathcal{H}^{3}}-\frac{2}{\mathcal{H}}\right) \Phi^{(1)}-\frac{\mathcal{H}^{\prime}}{\mathcal{H}^{3}} v_{\|}^{(1)}+\left(2 \frac{\bar{\chi}}{\mathcal{H}}+\frac{1}{\mathcal{H}^{2}}\right) \frac{\mathrm{d}}{\mathrm{d} \bar{\chi}} \Phi^{(1)}-\frac{1}{\mathcal{H}^{2}} \frac{\mathrm{d}}{\mathrm{d} \bar{\chi}} v_{\|}^{(1)}\right. \\
& \left.+\left(\frac{\bar{\chi}}{\mathcal{H}}+\frac{1}{\mathcal{H}^{2}}\right)\left(\Phi^{(1) \prime}+\Psi^{(1) \prime}\right)-2\left(\frac{\mathcal{H}^{\prime}}{\mathcal{H}^{3}}-\frac{1}{\mathcal{H}}\right) I^{(1)}\right\}-2 v_{\perp o}^{i(1)}\left[+\frac{\bar{\chi}}{\mathcal{H}} \partial_{\perp i}\left(\Phi^{(1)}+v_{\|}^{(1)}\right)-\frac{2}{\mathcal{H}} \int_{0}^{\bar{\chi}} \mathrm{d} \tilde{\chi} \tilde{\partial}_{\perp i} \Phi^{(1)}\right] \\
& -\frac{1}{\mathcal{H}} \Phi^{(2)}+\frac{1}{\mathcal{H}} v_{\|}^{(2)}+\left(-\frac{\mathcal{H}^{\prime}}{\mathcal{H}^{3}}+\frac{6}{\mathcal{H}}\right)\left(\Phi^{(1)}\right)^{2}-\frac{\mathcal{H}^{\prime}}{\mathcal{H}^{3}}\left(v_{\|}^{(1)}\right)^{2}+\frac{1}{\mathcal{H}} v_{\perp i}^{(1)} v_{\perp}^{i(1)}-\frac{2}{\mathcal{H}} v_{\|}^{(1)} \Psi^{(1)}+2 \frac{\mathcal{H}^{\prime}}{\mathcal{H}^{3}} \Phi^{(1)} v_{\|}^{(1)} \\
& -\frac{2}{\mathcal{H}^{2}}\left(\Phi^{(1)}-v_{\|}^{(1)}\right)\left[\frac{\mathrm{d}}{\mathrm{d} \bar{\chi}}\left(\Phi^{(1)}-v_{\|}^{(1)}\right)+\left(\Phi^{(1) \prime}+\Psi^{(1) \prime}\right)\right]-4\left[\left(\frac{2}{\mathcal{H}}-\frac{\mathcal{H}^{\prime}}{\mathcal{H}^{3}}\right) \Phi^{(1)}+\frac{\mathcal{H}^{\prime}}{\mathcal{H}^{3}} v_{\|}^{(1)}\right. \\
& \left.-\frac{1}{\mathcal{H}^{2}} \frac{\mathrm{d}}{\mathrm{d} \bar{\chi}}\left(\Phi^{(1)}-v_{\|}^{(1)}\right)-\frac{1}{\mathcal{H}^{2}}\left(\Phi^{(1) \prime}+\Psi^{(1) \prime}\right)+\left(\frac{\mathcal{H}^{\prime}}{\mathcal{H}^{3}}+\frac{1}{\mathcal{H}}\right) I^{(1)}\right] I^{(1)}+\frac{4}{\mathcal{H}} v_{\perp i}^{(1)} S_{\perp}^{i(1)}-\frac{2}{\mathcal{H}} \partial_{\|}\left(\Phi^{(1)}+v_{\|}^{(1)}\right) T^{(1)} \\
& -\frac{2}{\mathcal{H}}\left[2 \frac{\mathrm{d}}{\mathrm{d} \bar{\chi}} \Phi^{(1)}+\left(\Phi^{(1) \prime}+\Psi^{(1) \prime}\right)\right] \int_{0}^{\bar{\chi}} \mathrm{d} \tilde{\chi}\left[2 \Phi^{(1)}+(\bar{\chi}-\tilde{\chi})\left(\Phi^{(1) \prime}+\Psi^{(1) \prime}\right)\right]-\frac{2}{\mathcal{H}}\left[\partial_{\perp i}\left(\Phi^{(1)}+v_{\|}^{(1)}\right)\right. \\
& \left.-\frac{1}{\bar{\chi}} v_{\perp i}^{(1)}\right] \int_{0}^{\bar{\chi}} \mathrm{d} \tilde{\chi}\left[(\bar{\chi}-\tilde{\chi}) \tilde{\partial}_{\perp}^{i}\left(\Phi^{(1)}+\Psi^{(1)}\right)\right]+\frac{2}{\mathcal{H}} I^{(2)}-\frac{4}{\mathcal{H}} \int_{0}^{\bar{\chi}} \mathrm{d} \tilde{\chi}\left[\left(\Psi^{(1)}+2 I^{(1)}\right)\left(\Phi^{(1) \prime}+\Psi^{(1) \prime}\right)\right. \\
& \left.+\left(\Phi^{(1)}+\Psi^{(1)}\right) \frac{\mathrm{d}}{\mathrm{d} \tilde{\chi}} \Phi^{(1)}+2 S_{\perp}^{i(1)} \tilde{\partial}_{\perp i} \Phi^{(1)}\right]+\Delta x_{\text {post }- \text { Born }}^{0(2)} \\
& (211)
\end{aligned}
$$

where

$$
\begin{aligned}
& \Delta x_{\text {post-Born }}^{0(2)}=+\frac{4}{\mathcal{H}} \Phi_{o}^{(1)}\left(3 v_{\| o}^{(1)}-\Psi_{o}^{(1)}\right)+\frac{2}{\mathcal{H}}\left(\Phi_{o}^{(1)}-v_{\| o}^{(1)}\right)\left\{2 \Phi^{(1)}-2 I^{(1)}-\bar{\chi}\left[2 \frac{\mathrm{d}}{\mathrm{d} \bar{\chi}} \Phi^{(1)}+\left(\Phi^{(1) \prime}+\Psi^{(1) \prime}\right)\right]\right\} \\
& +\frac{8}{\mathcal{H}} v_{\| o}^{(1)} \int_{0}^{\bar{\chi}} \frac{\mathrm{d} \tilde{\chi}}{\tilde{\chi}} \Phi^{(1)}-\frac{4 \bar{\chi}}{\mathcal{H}} v_{\perp o}^{i(1)}\left\{-\partial_{\perp i} \Phi^{(1)}+\int_{0}^{\bar{\chi}} \mathrm{d} \tilde{\chi}\left(\tilde{\partial}_{\perp i} \Phi^{(1)}\right)\right\}-\frac{4}{\mathcal{H}} \Phi^{(1)}\left(\Phi^{(1)}-\Psi^{(1)}-2 I^{(1)}-2 \kappa^{(1)}\right) \\
& +\frac{2}{\mathcal{H}}\left[2 \frac{\mathrm{d}}{\mathrm{d} \bar{\chi}} \Phi^{(1)}+\left(\Phi^{(1) \prime}+\Psi^{(1) \prime}\right)\right] \int_{0}^{\bar{\chi}} \mathrm{d} \tilde{\chi}\left(\Phi^{(1)}-\Psi^{(1)}-2 I^{(1)}\right)+\frac{4}{\mathcal{H}} \Phi^{(1) \prime} T^{(1)}-\frac{8}{\mathcal{H}} \partial_{\perp i} \Phi^{(1)} \int_{0}^{\bar{\chi}} \mathrm{d} \tilde{\chi} S_{\perp}^{i(1)} \\
& +\frac{2}{\mathcal{H}} \int_{0}^{\bar{\chi}} \mathrm{d} \tilde{\chi}\left\{\left(\Phi^{(1) \prime \prime}+\Psi^{(1) \prime \prime}\right) T^{(1)}+2 \Phi^{(1) \prime}\left(\Phi^{(1)}+\Psi^{(1)}\right)-\left(\Phi^{(1) \prime}+\Psi^{(1) \prime}\right)\left(\Phi^{(1)}-\Psi^{(1)}-2 I^{(1)}\right)\right. \\
& +2 \Phi^{(1)}\left[\frac{\mathrm{d}}{\mathrm{d} \bar{\chi}}\left(\Phi^{(1)}-\Psi^{(1)}\right)+\left(\Phi^{(1) \prime}+\Psi^{(1) \prime}\right)\right]+4 S_{\perp}^{i(1)} \tilde{\partial}_{\perp i} \Phi^{(1)}+4 \Phi^{(1)} \tilde{\partial}_{\perp j} S_{\perp}^{j(1)}-4\left(\frac{\mathrm{d}}{\mathrm{d} \tilde{\chi}} \Phi^{(1)}-\frac{1}{\tilde{\chi}} \Phi^{(1)}\right) \kappa^{(1)} \\
& \left.-\tilde{\partial}_{\perp i}\left(\Phi^{(1) \prime}+\Psi^{(1) \prime}\right) \delta x_{\perp}^{i(1)}\right\} .
\end{aligned}
$$

From Eqs. (71) and (116) we deduce

$$
\begin{aligned}
\Delta x_{\|}^{(2)}= & \bar{\chi}\left[\left(\Phi_{o}^{(1)}\right)^{2}+2 \Phi_{o}^{(1)} v_{\| o}^{(1)}+2 v_{\| o}^{(1)} \Psi_{o}^{(1)}-\left(\Psi_{o}^{(1)}\right)^{2}-v_{\perp k o}^{(1)} v_{\perp o}^{k(1)}\right]-2\left(\Phi_{o}^{(1)}-v_{\| o}^{(1)}\right)\left[\bar{\chi}\left(\Phi^{(1)}+\Psi^{(1)}\right)+2 T^{(1)}\right] \\
& -4 v_{\perp o}^{i(1)} \int_{0}^{\bar{\chi}} \mathrm{d} \tilde{\chi}(\bar{\chi}-\tilde{\chi})\left[\tilde{\partial}_{\perp}^{i}\left(\Phi^{(1)}+\Psi^{(1)}\right)\right]+2\left(\Phi^{(1)}+\Psi^{(1)}\right) \int_{0}^{\bar{\chi}} \mathrm{d} \tilde{\chi}\left[2 \Phi^{(1)}+(\bar{\chi}-\tilde{\chi})\left(\Phi^{(1) \prime}+\Psi^{(1) \prime}\right)\right] \\
& -T^{(2)}+4 \int_{0}^{\bar{\chi}} \mathrm{d} \tilde{\chi}\left[-2\left(\Phi^{(1)}\right)^{2}-\Psi^{(1)}\left(\Phi^{(1)}-\Psi^{(1)}\right)+2\left(\Phi^{(1)}+\Psi^{(1)}\right) I^{(1)}-2 S_{\perp}^{i(1)} S_{\perp}^{j(1)} \delta_{i j}\right] \\
& +4 \int_{0}^{\bar{\chi}} \mathrm{d} \tilde{\chi}\left\{(\bar{\chi}-\tilde{\chi})\left(\Phi^{(1)}+\Psi^{(1)}\right)\left[\left(\Phi^{(1) \prime}+\Psi^{(1) \prime}\right)+\frac{\mathrm{d}}{\mathrm{d} \tilde{\chi}} \Phi^{(1)}\right]\right\}-\frac{2}{\mathcal{H}}\left(\Phi^{(1)}+\Psi^{(1)}\right) \Delta \ln a^{(1)} \\
& -\frac{1}{\mathcal{H}} \Delta \ln a^{(2)}+\left(\frac{\mathcal{H}^{\prime}}{\mathcal{H}^{3}}+\frac{1}{\mathcal{H}}\right)\left(\Delta \ln a^{(1)}\right)^{2}+\left(\delta x^{0(2)}+\delta x_{\|}^{(2)}\right)_{\text {post-Born }},
\end{aligned}
$$


and from Eqs. 72 and 115 we find

$$
\begin{aligned}
\Delta x_{\perp}^{i(2)}= & \bar{\chi}\left[-2 \omega_{\perp o}^{i(2)}-v_{\perp o}^{i(2)}+\frac{1}{2} n^{j} \hat{h}_{j k o}^{(2)} \mathcal{P}^{k i}+2 \Phi_{o}^{(1)} v_{\perp o}^{i(1)}-v_{\| o}^{(1)} v_{\perp o}^{i(1)}+4 v_{\perp o}^{i(1)} \Psi_{o}^{(1)}\right]-4 \bar{\chi}\left(\Phi_{o}^{(1)}-v_{\| o}^{(1)}\right) S_{\perp}^{i(1)} \\
& -4\left(\Phi_{o}^{(1)}-v_{\| o}^{(1)}\right) \int_{0}^{\bar{\chi}} \mathrm{d} \tilde{\chi}\left[(\bar{\chi}-\tilde{\chi}) \tilde{\partial}_{\perp}^{i}\left(\Phi^{(1)}+\Psi^{(1)}\right)\right]-2 v_{\perp o}^{i(1)}\left\{\int _ { 0 } ^ { \overline { \chi } } \mathrm { d } \tilde { \chi } \left[2\left(\Phi^{(1)}+\Psi^{(1)}\right)\right.\right. \\
& \left.\left.+(\bar{\chi}-\tilde{\chi})\left(\Phi^{(1) \prime}+\Psi^{(1) \prime}\right)\right]-\frac{1}{\mathcal{H}} \Delta \ln a^{(1)}\right\}-\frac{4}{\mathcal{H}} S_{\perp}^{i(1)} \Delta \ln a^{(1)}+4 S_{\perp}^{i(1)} \int_{0}^{\bar{\chi}} \mathrm{d} \tilde{\chi}\left[2 \Phi^{(1)}\right. \\
& \left.+(\bar{\chi}-\tilde{\chi})\left(\Phi^{(1) \prime}+\Psi^{(1) \prime}\right)\right]+\int_{0}^{\bar{\chi}} \mathrm{d} \tilde{\chi}\left\{2 \omega_{\perp}^{i(2)}-n^{j} \hat{h}_{j k}^{(2)} \mathcal{P}^{k i}+8 \Psi^{(1)} S_{\perp}^{i(1)}\right\} \\
& +\int_{0}^{\bar{\chi}} \mathrm{d} \tilde{\chi}(\bar{\chi}-\tilde{\chi})\left\{-\left[\tilde{\partial}_{\perp}^{i}\left(\Phi^{(2)}+2 \omega_{\|}^{(2)}+\Psi^{(2)}-\frac{1}{2} \hat{h}_{\|}^{(2)}\right)+\frac{1}{\tilde{\chi}}\left(-2 \omega_{\perp}^{i(2)}+n^{k} \hat{h}_{k j}^{(2)} \mathcal{P}^{i j}\right)\right]\right. \\
& \left.+8\left(\Phi^{(1)}-I^{(1)}\right) \tilde{\partial}_{\perp}^{i}\left(\Phi^{(1)}+\Psi^{(1)}\right)-4\left(\Phi^{(1)}+\Psi^{(1)}\right) \tilde{\partial}_{\perp}^{i} \Psi^{(1)}\right\}+\delta x_{\perp \text { post-Born }}^{i(2)} .
\end{aligned}
$$

To obtain explicitly Eq. 132 we need

$$
\begin{aligned}
& \left(\frac{\mathrm{d} \Delta \ln a}{\mathrm{~d} \bar{\chi}}\right)^{(2)}=+2\left(\Phi_{o}^{(1)}-v_{\| o}^{(1)}\right)\left\{\left(\frac{\mathcal{H}^{\prime}}{\mathcal{H}^{2}}-1\right)\left[\frac{\mathrm{d}}{\mathrm{d} \bar{\chi}}\left(\Phi^{(1)}-v_{\|}^{(1)}\right)+\left(\Phi^{(1) \prime}+\Psi^{(1) \prime}\right)\right]+\frac{1}{\mathcal{H}} \frac{\mathrm{d}}{\mathrm{d} \bar{\chi}}\left[\frac{\mathrm{d}}{\mathrm{d} \bar{\chi}}\left(\Phi^{(1)}-v_{\|}^{(1)}\right)\right.\right. \\
& \left.\left.+\left(\Phi^{(1) \prime}+\Psi^{(1) \prime}\right)\right]+\bar{\chi} \frac{\mathrm{d}}{\mathrm{d} \bar{\chi}}\left[2 \frac{\mathrm{d}}{\mathrm{d} \bar{\chi}} \Phi^{(1)}+\left(\Phi^{(1) \prime}+\Psi^{(1) \prime}\right)\right]\right\}-2 v_{\perp o}^{i(1)}\left[-\partial_{\perp i}\left(\Phi^{(1)}-v_{\|}^{(1)}\right)+\bar{\chi} \frac{\mathrm{d}}{\mathrm{d} \bar{\chi}} \partial_{\perp i}\left(\Phi^{(1)}+v_{\|}^{(1)}\right)\right] \\
& +\frac{\mathrm{d}}{\mathrm{d} \bar{\chi}}\left(-\Phi^{(2)}+v_{\|}^{(2)}\right)-\left(\Phi^{(2) \prime}+2 \omega_{\|}^{(2) \prime}+\Psi^{(2) \prime}-\frac{1}{2} \hat{h}_{\|}^{(2) \prime}\right)+\frac{\mathrm{d}}{\mathrm{d} \bar{\chi}}\left[7\left(\Phi^{(1)}\right)^{2}-2 \Phi^{(1)} v_{\|}^{(1)}+\left(v_{\|}^{(1)}\right)^{2}-2 v_{\|}^{(1)} \Psi^{(1)}\right] \\
& -4\left[2 \frac{\mathrm{d}}{\mathrm{d} \bar{\chi}} \Phi^{(1)}+\left(\Phi^{(1) \prime}+\Psi^{(1) \prime}\right)\right] \Phi^{(1)}-2\left\{\frac{\mathcal{H}^{\prime}}{\mathcal{H}^{2}}\left(\Phi^{(1)}-v_{\|}^{(1)}\right)+\frac{1}{\mathcal{H}}\left[\frac{\mathrm{d}}{\mathrm{d} \bar{\chi}}\left(\Phi^{(1)}-v_{\|}^{(1)}\right)+\left(\Phi^{(1) \prime}+\Psi^{(1) \prime}\right)\right]\right\} \\
& \times\left[\frac{\mathrm{d}}{\mathrm{d} \bar{\chi}}\left(\Phi^{(1)}-v_{\|}^{(1)}\right)+\left(\Phi^{(1) \prime}+\Psi^{(1) \prime}\right)\right]+2\left[3 \Phi^{(1)}-2 \Psi^{(1)}-v_{\|}^{(1)}\right]\left(\Phi^{(1) \prime}+\Psi^{(1) \prime}\right) \\
& -\frac{2}{\mathcal{H}}\left(\Phi^{(1)}-v_{\|}^{(1)}\right) \frac{\mathrm{d}}{\mathrm{d} \bar{\chi}}\left[\frac{\mathrm{d}}{\mathrm{d} \bar{\chi}}\left(\Phi^{(1)}-v_{\|}^{(1)}\right)+\left(\Phi^{(1) \prime}+\Psi^{(1) \prime}\right)\right]+2\left(\Phi^{(1)}+\Psi^{(1)}\right)\left[-2 \frac{\mathrm{d}}{\mathrm{d} \bar{\chi}} \Phi^{(1)}+\partial_{\|}\left(\Phi^{(1)}+v_{\|}^{(1)}\right)\right] \\
& -2 v_{\perp i}^{(1)} \partial_{\perp}^{i}\left(\Phi^{(1)}+\Psi^{(1)}\right)+2 v_{\perp i}^{(1)} \frac{\mathrm{d}}{\mathrm{d} \bar{\chi}} v_{\perp}^{i(1)}+4\left\{\left(\frac{\mathcal{H}^{\prime}}{\mathcal{H}^{2}}-1\right)\left[\frac{\mathrm{d}}{\mathrm{d} \bar{\chi}}\left(\Phi^{(1)}-v_{\|}^{(1)}\right)+\left(\Phi^{(1) \prime}+\Psi^{(1) \prime}\right)\right]\right. \\
& +\frac{1}{\mathcal{H}} \frac{\mathrm{d}}{\mathrm{d} \bar{\chi}}\left[\frac{\mathrm{d}}{\mathrm{d} \bar{\chi}}\left(\Phi^{(1)}-v_{\|}^{(1)}\right)+\left(\Phi^{(1) \prime}+\Psi^{(1) \prime}\right)\right] I^{(1)}-2\left[\frac{\mathrm{d}}{\mathrm{d} \bar{\chi}} \partial_{\|}\left(\Phi^{(1)}+v_{\|}^{(1)}\right)\right] T^{(1)}-2 \frac{\mathrm{d}}{\mathrm{d} \bar{\chi}}\left[2 \frac{\mathrm{d}}{\mathrm{d} \bar{\chi}} \Phi^{(1)}\right. \\
& \left.+\left(\Phi^{(1) \prime}+\Psi^{(1) \prime}\right)\right] \int_{0}^{\bar{\chi}} \mathrm{d} \tilde{\chi}\left[2 \Phi^{(1)}+(\bar{\chi}-\tilde{\chi})\left(\Phi^{(1) \prime}+\Psi^{(1) \prime}\right)\right]-4\left[\partial_{\perp i}\left(\Phi^{(1)}-v_{\|}^{(1)}\right)+\frac{1}{\tilde{\chi}} v_{\perp i}^{(1)}-\frac{\mathrm{d}}{\mathrm{d} \bar{\chi}} v_{\perp i}^{(1)}\right] S_{\perp}^{i(1)} \\
& -2 \frac{\mathrm{d}}{\mathrm{d} \bar{\chi}}\left[\partial_{\perp i}\left(\Phi^{(1)}+v_{\|}^{(1)}\right)-\frac{1}{\bar{\chi}} v_{\perp i}^{(1)}\right] \int_{0}^{\bar{\chi}} \mathrm{d} \tilde{\chi}\left[(\bar{\chi}-\tilde{\chi}) \tilde{\partial}_{\perp}^{i}\left(\Phi^{(1)}+\Psi^{(1)}\right)\right]+\left(\frac{\mathrm{d} \Delta \ln a}{\mathrm{~d} \bar{\chi}}\right)
\end{aligned}
$$

where

$$
\begin{aligned}
\left(\frac{\mathrm{d} \Delta \ln a}{\mathrm{~d} \bar{\chi}}\right)_{\text {post-Born }}^{(2)}= & -2 \bar{\chi}\left(\Phi_{o}^{(1)}-v_{\| o}^{(1)}\right) \frac{\mathrm{d}}{\mathrm{d} \bar{\chi}}\left[2 \frac{\mathrm{d}}{\mathrm{d} \bar{\chi}} \Phi^{(1)}+\left(\Phi^{(1) \prime}+\Psi^{(1) \prime}\right)\right] \\
& +2 \bar{\chi} v_{\perp o}^{i(1)}\left[2 \partial_{\perp i} \frac{\mathrm{d}}{\mathrm{d} \bar{\chi}} \Phi^{(1)}+\partial_{\perp i}\left(\Phi^{(1) \prime}+\Psi^{(1) \prime}\right)-\frac{2}{\bar{\chi}} \partial_{\perp i} \Phi^{(1)}\right] \\
& +2 \frac{\mathrm{d}}{\mathrm{d} \bar{\chi}}\left[2 \frac{\mathrm{d}}{\mathrm{d} \bar{\chi}} \Phi^{(1)}+\left(\Phi^{(1) \prime}+\Psi^{(1) \prime}\right)\right] \int_{0}^{\bar{\chi}} \mathrm{d} \tilde{\chi}\left(\Phi^{(1)}-\Psi^{(1)}-2 I^{(1)}\right) \\
& +2\left[2 \frac{\mathrm{d}}{\mathrm{d} \bar{\chi}} \Phi^{(1) \prime}+\left(\Phi^{(1) \prime \prime}+\Psi^{(1) \prime \prime}\right)\right] T^{(1)} \\
& -4\left[2 \partial_{\perp i} \frac{\mathrm{d}}{\mathrm{d} \bar{\chi}} \Phi^{(1)}+\partial_{\perp i}\left(\Phi^{(1) \prime}+\Psi^{(1) \prime}\right)-\frac{2}{\bar{\chi}} \partial_{\perp i} \Phi^{(1)}\right] \int_{0}^{\bar{\chi}} \mathrm{d} \tilde{\chi} S_{\perp}^{i(1)} .
\end{aligned}
$$


Then we obtain

$$
\begin{aligned}
& \partial_{\|} \Delta x_{\|}^{(2)}=\left(\Phi_{o}^{(1)}\right)^{2}+2 \Phi_{o}^{(1)} v_{\| o}^{(1)}+2 v_{\| o}^{(1)} \Psi_{o}^{(1)}-\left(\Psi_{o}^{(1)}\right)^{2}-v_{\perp k o}^{(1)} v_{\perp o}^{k(1)}+2\left(\Phi_{o}^{(1)}-v_{\| o}^{(1)}\right) \\
& \times\left\{\left(-\frac{\mathcal{H}^{\prime}}{\mathcal{H}^{3}}+\frac{1}{\mathcal{H}}\right)\left[\frac{\mathrm{d}}{\mathrm{d} \bar{\chi}}\left(\Phi^{(1)}-v_{\|}^{(1)}\right)+\left(\Phi^{(1) \prime}+\Psi^{(1) \prime}\right)\right]-\frac{\bar{\chi}}{\mathcal{H}} \frac{\mathrm{d}}{\mathrm{d} \bar{\chi}}\left[2 \frac{\mathrm{d}}{\mathrm{d} \bar{\chi}} \Phi^{(1)}+\left(\Phi^{(1) \prime}+\Psi^{(1) \prime}\right)\right]\right. \\
& \left.+\left(\Phi^{(1)}+\Psi^{(1)}\right)-\bar{\chi} \frac{\mathrm{d}}{\mathrm{d} \bar{\chi}}\left(\Phi^{(1)}+\Psi^{(1)}\right)-\frac{1}{\mathcal{H}^{2}} \frac{\mathrm{d}}{\mathrm{d} \bar{\chi}}\left[\frac{\mathrm{d}}{\mathrm{d} \bar{\chi}}\left(\Phi^{(1)}-v_{\|}^{(1)}\right)+\left(\Phi^{(1) \prime}+\Psi^{(1) \prime}\right)\right]\right\}-2 v_{\perp o}^{i(1)} \\
& \times\left[+\frac{1}{\mathcal{H}} \partial_{\perp i}\left(\Phi^{(1)}-v_{\|}^{(1)}\right)-2 \frac{\bar{\chi}}{\mathcal{H}} \frac{\mathrm{d}}{\mathrm{d} \bar{\chi}} \partial_{\perp i}\left(\Phi^{(1)}+v_{\|}^{(1)}\right)-4 \delta_{i l} S_{\perp}^{l(1)}\right]+\Phi^{(2)}+2 \omega_{\|}^{(2)}+\Psi^{(2)}-\frac{1}{2} \hat{h}_{\|}^{(2)} \\
& +\frac{1}{\mathcal{H}}\left[\frac{\mathrm{d}}{\mathrm{d} \bar{\chi}}\left(\Phi^{(2)}-v_{\|}^{(2)}\right)+\left(\Phi^{(2) \prime}+2 \omega_{\|}^{(2) \prime}+\Psi^{(2) \prime}-\frac{1}{2} \hat{h}_{\|}^{(2) \prime}\right)\right]-4\left(\Phi^{(1)}-\Psi^{(1)}\right)\left(\Phi^{(1)}+\Psi^{(1)}\right)-\frac{1}{\mathcal{H}} \frac{\mathrm{d}}{\mathrm{d} \bar{\chi}}\left[7\left(\Phi^{(1)}\right)^{2}\right. \\
& \left.-2 \Phi^{(1)} v_{\|}^{(1)}+\left(v_{\|}^{(1)}\right)^{2}-2 v_{\|}^{(1)} \Psi^{(1)}+v_{\perp i}^{(1)} v_{\perp}^{i(1)}\right]+\frac{2}{\mathcal{H}^{2}}\left(\Phi^{(1)}-v_{\|}^{(1)}\right) \frac{\mathrm{d}}{\mathrm{d} \bar{\chi}}\left[\frac{\mathrm{d}}{\mathrm{d} \bar{\chi}}\left(\Phi^{(1)}-v_{\|}^{(1)}\right)+\left(\Phi^{(1) \prime}+\Psi^{(1) \prime}\right)\right] \\
& -\frac{4}{\mathcal{H}}\left[\left(\Phi^{(1)}-\Psi^{(1)}\right)+\left(\Phi^{(1)}-v_{\|}^{(1)}\right)\right]\left(\Phi^{(1) \prime}+\Psi^{(1) \prime}\right)+\frac{4}{\mathcal{H}} \Phi^{(1)}\left[2 \frac{\mathrm{d}}{\mathrm{d} \bar{\chi}} \Phi^{(1)}+\left(\Phi^{(1) \prime}+\Psi^{(1) \prime}\right)\right] \\
& +2\left\{\frac{1}{\mathcal{H}}\left(\Phi^{(1)}+\Psi^{(1)}\right)+\frac{\mathcal{H}^{\prime}}{\mathcal{H}^{3}}\left(\Phi^{(1)}-v_{\|}^{(1)}\right)+\frac{1}{\mathcal{H}^{2}}\left[\frac{\mathrm{d}}{\mathrm{d} \bar{\chi}}\left(\Phi^{(1)}-v_{\|}^{(1)}\right)+\left(\Phi^{(1) \prime}+\Psi^{(1) \prime}\right)\right]\right\} \\
& \times\left[\frac{\mathrm{d}}{\mathrm{d} \bar{\chi}}\left(\Phi^{(1)}-v_{\|}^{(1)}\right)+\left(\Phi^{(1) \prime}+\Psi^{(1) \prime}\right)\right]+\frac{2}{\mathcal{H}}\left[2 \frac{\mathrm{d}}{\mathrm{d} \bar{\chi}} \Phi^{(1)}-\partial_{\|}\left(\Phi^{(1)}+v_{\|}^{(1)}\right)\right]\left(\Phi^{(1)}+\Psi^{(1)}\right) \\
& +\frac{2}{\mathcal{H}} v_{\perp i}^{(1)} \partial_{\perp}^{i}\left(\Phi^{(1)}+\Psi^{(1)}\right)+\frac{2}{\mathcal{H}} \frac{\mathrm{d}}{\mathrm{d} \bar{\chi}} \partial_{\|}\left(\Phi^{(1)}+v_{\|}^{(1)}\right) T^{(1)}+4\left\{\left(-\frac{\mathcal{H}^{\prime}}{\mathcal{H}^{3}}+\frac{1}{\mathcal{H}}\right)\left[\frac{\mathrm{d}}{\mathrm{d} \bar{\chi}}\left(\Phi^{(1)}-v_{\|}^{(1)}\right)+\left(\Phi^{(1) \prime}+\Psi^{(1) \prime}\right)\right]\right. \\
& \left.-\frac{1}{\mathcal{H}^{2}} \frac{\mathrm{d}}{\mathrm{d} \bar{\chi}}\left[\frac{\mathrm{d}}{\mathrm{d} \bar{\chi}}\left(\Phi^{(1)}-v_{\|}^{(1)}\right)+\left(\Phi^{(1) \prime}+\Psi^{(1) \prime}\right)\right]+\left(\Phi^{(1)}+\Psi^{(1)}\right)\right\} I^{(1)}+2\left\{\frac{\mathrm{d}}{\mathrm{d} \bar{\chi}}\left(\Phi^{(1)}+\Psi^{(1)}\right)\right. \\
& \left.+\frac{1}{\mathcal{H}} \frac{\mathrm{d}}{\mathrm{d} \bar{\chi}}\left[2 \frac{\mathrm{d}}{\mathrm{d} \bar{\chi}} \Phi^{(1)}+\left(\Phi^{(1) \prime}+\Psi^{(1) \prime}\right)\right]\right\} \int_{0}^{\bar{\chi}} \mathrm{d} \tilde{\chi}\left[2 \Phi^{(1)}+(\bar{\chi}-\tilde{\chi})\left(\Phi^{(1) \prime}+\Psi^{(1) \prime}\right)\right]+4\left[\frac{1}{\mathcal{H}} \partial_{\perp i}\left(\Phi^{(1)}-v_{\|}^{(1)}\right)\right. \\
& \left.+\frac{1}{\mathcal{H} \bar{\chi}} v_{\perp i}^{(1)}-\frac{1}{\mathcal{H}} \frac{\mathrm{d}}{\mathrm{d} \bar{\chi}} v_{\perp i}^{(1)}-2 S_{\perp}^{j(1)} \delta_{i j}\right] S_{\perp}^{i(1)}+\frac{2}{\mathcal{H}} \frac{\mathrm{d}}{\mathrm{d} \bar{\chi}}\left[\partial_{\perp i}\left(\Phi^{(1)}+v_{\|}^{(1)}\right)-\frac{1}{\bar{\chi}} v_{\perp i}^{(1)}\right] \int_{0}^{\bar{\chi}} \mathrm{d} \tilde{\chi}\left[(\bar{\chi}-\tilde{\chi}) \tilde{\partial}_{\perp}^{i}\left(\Phi^{(1)}+\Psi^{(1)}\right)\right] \\
& -\frac{\mathcal{H}^{\prime}}{\mathcal{H}^{2}} \Delta \ln a^{(2)}+\left[-\frac{\mathcal{H}^{\prime \prime}}{\mathcal{H}^{3}}+3\left(\frac{\mathcal{H}^{\prime}}{\mathcal{H}^{2}}\right)^{2}+\frac{\mathcal{H}^{\prime}}{\mathcal{H}^{2}}\right]\left(\Delta \ln a^{(1)}\right)^{2}+4 \int_{0}^{\bar{\chi}} \mathrm{d} \tilde{\chi}\left[\left(\Phi^{(1)}+\Psi^{(1)}\right)\left(\Phi^{(1) \prime}+\Psi^{(1) \prime}+\frac{\mathrm{d}}{\mathrm{d} \tilde{\chi}} \Phi^{(1)}\right)\right] \\
& +2\left\{-\frac{\mathcal{H}^{\prime}}{\mathcal{H}^{2}}\left(\Phi^{(1)}+\Psi^{(1)}\right)-\frac{1}{\mathcal{H}} \frac{\mathrm{d}}{\mathrm{d} \bar{\chi}}\left(\Phi^{(1)}+\Psi^{(1)}\right)-\left(\frac{\mathcal{H}^{\prime}}{\mathcal{H}^{3}}+\frac{1}{\mathcal{H}}\right)\left[\frac{\mathrm{d}}{\mathrm{d} \bar{\chi}}\left(\Phi^{(1)}-v_{\|}^{(1)}\right)+\left(\Phi^{(1) \prime}+\Psi^{(1) \prime}\right)\right]\right\} \Delta \ln a^{(1)} \\
& +\partial_{\|} \Delta x_{\| \text {post-Born }}^{(2)} \text {, }
\end{aligned}
$$


where

$$
\begin{aligned}
& \partial_{\|} \Delta x_{\| \text {post-Born }}^{(2)}=-2\left(\Phi_{o}^{(1)}+\Psi_{o}^{(1)}\right)\left(3 v_{\| o}^{(1)}-\Psi_{o}^{(1)}\right)+2\left(\Phi_{o}^{(1)}-v_{\| o}^{(1)}\right)\left\{-\left(\Phi^{(1)}+\Psi^{(1)}\right)+\frac{\bar{\chi}}{\mathcal{H}} \frac{\mathrm{d}}{\mathrm{d} \bar{\chi}}\left[2 \frac{\mathrm{d}}{\mathrm{d} \bar{\chi}} \Phi^{(1)}\right.\right. \\
& \left.\left.+\Phi^{(1) \prime}+\Psi^{(1) \prime}\right]\right\}+2 \bar{\chi}\left(\Phi_{o}^{(1)}-v_{\| o}^{(1)}\right) \frac{\mathrm{d}}{\mathrm{d} \bar{\chi}}\left(\Phi^{(1)}+\Psi^{(1)}\right)-2 v_{\perp o}^{i(1)}\left\{\bar{\chi} \partial_{\perp i}\left(\Phi^{(1)}+\Psi^{(1)}\right)-\frac{2}{\mathcal{H}} \partial_{\perp i} \Phi^{(1)}+2 \frac{\bar{\chi}}{\mathcal{H}} \partial_{\perp i} \frac{\mathrm{d}}{\mathrm{d} \bar{\chi}} \Phi^{(1)}\right. \\
& \left.+\frac{\bar{\chi}}{\mathcal{H}} \partial_{\perp i}\left(\Phi^{(1) \prime}+\Psi^{(1) \prime}\right)+2 S_{\perp}^{j(1)}\right\}-4 v_{\| o}^{(1)} \int_{0}^{\bar{\chi}} \frac{\mathrm{d} \tilde{\chi}}{\tilde{\chi}}\left(\Phi^{(1)}+\Psi^{(1)}\right)-2\left[\left(\Phi^{(1) \prime}+\Psi^{(1) \prime}\right)\right. \\
& \left.+\frac{1}{\mathcal{H}}\left(2 \frac{\mathrm{d}}{\mathrm{d} \bar{\chi}} \Phi^{(1) \prime}+\Phi^{(1) \prime \prime}+\Psi^{(1) \prime \prime}\right)\right] T^{(1)}-2\left\{\frac{\mathrm{d}}{\mathrm{d} \bar{\chi}}\left(\Phi^{(1)}+\Psi^{(1)}\right)+\frac{1}{\mathcal{H}} \frac{\mathrm{d}}{\mathrm{d} \bar{\chi}}\left[2 \frac{\mathrm{d}}{\mathrm{d} \bar{\chi}} \Phi^{(1)}+\left(\Phi^{(1) \prime}+\Psi^{(1) \prime}\right)\right]\right\} \\
& \times \int_{0}^{\bar{\chi}} \mathrm{d} \tilde{\chi}\left(\Phi^{(1)}-\Psi^{(1)}-2 I^{(1)}\right)+2\left(\Phi^{(1)}+\Psi^{(1)}\right)\left(\Phi^{(1)}-\Psi^{(1)}-2 I^{(1)}-2 \kappa^{(1)}\right) \\
& +4\left[\partial_{\perp i}\left(\Phi^{(1)}+\Psi^{(1)}\right)+\frac{2}{\mathcal{H}} \partial_{\perp i} \frac{\mathrm{d}}{\mathrm{d} \bar{\chi}} \Phi^{(1)}+\frac{1}{\mathcal{H}} \partial_{\perp i}\left(\Phi^{(1) \prime}+\Psi^{(1) \prime}\right)-\frac{2}{\bar{\chi} \mathcal{H}} \partial_{\perp i} \Phi^{(1)}\right] \int_{0}^{\bar{\chi}} \mathrm{d} \tilde{\chi} S_{\perp}^{(i)} \\
& +2 \int_{0}^{\bar{\chi}} \mathrm{d} \tilde{\chi}\left\{-\left(\Phi^{(1)}+\Psi^{(1)}\right)\left[\frac{\mathrm{d}}{\mathrm{d} \tilde{\chi}}\left(\Phi^{(1)}-\Psi^{(1)}\right)+2\left(\Phi^{(1) \prime}+\Psi^{(1) \prime}\right)\right]-2 \tilde{\partial}_{\perp j}\left(\Phi^{(1)}+\Psi^{(1)}\right) S_{\perp}^{j(1)}\right. \\
& \left.-2\left(\Phi^{(1)}+\Psi^{(1)}\right) \tilde{\partial}_{\perp m} S_{\perp}^{m(1)}+2\left[\frac{\mathrm{d}}{\mathrm{d} \bar{\chi}}\left(\Phi^{(1)}+\Psi^{(1)}\right)-\frac{1}{\tilde{\chi}}\left(\Phi^{(1)}+\Psi^{(1)}\right)\right] \kappa^{(1)}\right\} \cdot
\end{aligned}
$$

From Eq. (131) and using Eqs. 125, (126) and (127), we obtain the coordinate convergence lensing term at second order

$$
\kappa^{(2)}=-\frac{1}{2} \partial_{\perp i} \Delta x_{\perp}^{i(2)}=\kappa_{1}^{(2)}+\kappa_{2}^{(2)}+\kappa_{3}^{(2)}+\kappa_{4}^{(2)}+\kappa_{\text {post-Born }}^{(2)}
$$

where

$$
\begin{aligned}
& \kappa_{1}^{(2)}=\frac{1}{2} \int_{0}^{\bar{\chi}} \mathrm{d} \tilde{\chi}(\bar{\chi}-\tilde{\chi}) \frac{\tilde{\chi}}{\bar{\chi}} \tilde{\nabla}_{\perp}^{2}\left(\Phi^{(2)}+2 \omega_{\|}^{(2)}+\Psi^{(2)}-\frac{1}{2} \hat{h}_{\|}^{(2)}\right), \\
& \kappa_{2}^{(2)}=\frac{1}{2} \int_{0}^{\bar{\chi}} \mathrm{d} \tilde{\chi}\left(-2 \tilde{\partial}_{\perp}^{i} \omega_{i}^{(2)}+\frac{4}{\tilde{\chi}} \omega_{\|}^{(2)}+\mathcal{P}^{i j} n^{k} \tilde{\partial}_{i} \hat{h}_{j k}^{(2)}-\frac{3}{\tilde{\chi}} \hat{h}_{\|}^{(2)}\right), \\
& \kappa_{3}^{(2)}=\left[\int_{0}^{\bar{\chi}} \mathrm{d} \tilde{\chi} \frac{\tilde{\chi}}{\bar{\chi}} \tilde{\nabla}_{\perp}^{2}\left(\Phi^{(1)}+\Psi^{(1)}\right)\right]\left\{\int_{0}^{\bar{\chi}} \mathrm{d} \tilde{\chi}\left[2 \Phi^{(1)}+(\bar{\chi}-\tilde{\chi})\left(\Phi^{(1) \prime}+\Psi^{(1) \prime}\right)\right]-\frac{1}{\mathcal{H}} \Delta \ln a^{(1)}\right\} \\
& -2 S_{\perp}^{i(1)}\left\{\int_{0}^{\bar{\chi}} \mathrm{d} \tilde{\chi} \frac{\tilde{\chi}}{\bar{\chi}}\left[2 \tilde{\partial}_{\perp i} \Phi^{(1)}+(\bar{\chi}-\tilde{\chi}) \tilde{\partial}_{\perp i}\left(\Phi^{(1) \prime}+\Psi^{(1) \prime}\right)\right]-\frac{1}{\mathcal{H}} \partial_{\perp i} \Delta \ln a^{(1)}\right\} \\
& +\int_{0}^{\bar{\chi}} \mathrm{d} \tilde{\chi} \frac{\tilde{\chi}}{\bar{\chi}}\left[-4 \tilde{\partial}_{\perp j} \Psi^{(1)} S_{\perp}^{j(1)}+\frac{8}{\tilde{\chi}} \Psi^{(1)} S_{\|}^{(1)}-4 \Psi^{(1)} \tilde{\partial}_{\perp m} S^{m(1)}\right] \\
& +2 \int_{0}^{\bar{\chi}} \mathrm{d} \tilde{\chi}\left\{( \overline { \chi } - \tilde { \chi } ) \frac { \tilde { \chi } } { \overline { \chi } } \left[\tilde{\partial}_{\perp}^{i} \Psi^{(1)} \tilde{\partial}_{\perp i}\left(\Phi^{(1)}+\Psi^{(1)}\right)+\left(\Phi^{(1)}+\Psi^{(1)}\right) \tilde{\nabla}_{\perp}^{2} \Psi^{(1)}\right.\right. \\
& \left.\left.-2 \tilde{\partial}_{\perp i}\left(\Phi^{(1)}-I^{(1)}\right) \tilde{\partial}_{\perp}^{i}\left(\Phi^{(1)}+\Psi^{(1)}\right)-2\left(\Phi^{(1)}-I^{(1)}\right) \tilde{\nabla}_{\perp}^{2}\left(\Phi^{(1)}+\Psi^{(1)}\right)\right]\right\}
\end{aligned}
$$




$$
\begin{aligned}
\kappa_{4}^{(2)}= & -2 \omega_{\| o}^{(2)}-v_{\| o}^{(2)}+\frac{3}{4} \hat{h}_{\| o}^{(2)}-\frac{1}{4} \hat{h}_{i o}^{i(2)}+2 \Phi_{o}^{(1)} v_{\| o}^{(1)}+\frac{1}{2} v_{\perp i o}^{(1)} v_{\perp o}^{i(1)}-\left(v_{\| o}^{(1)}\right)^{2}+4 v_{\| o}^{(1)} \Psi_{o}^{(1)} \\
& +\left(\Phi_{o}^{(1)}-v_{\| o}^{(1)}\right)\left\{-\int_{0}^{\bar{\chi}} \mathrm{d} \tilde{\chi}\left[\tilde{\chi} \tilde{\nabla}_{\perp}^{2}\left(\Phi^{(1)}+\Phi^{(1)}\right)\right]+4 \kappa_{2}^{(1)}+4 \kappa_{1}^{(1)}\right\} \\
& -\frac{2}{\bar{\chi}} v_{\| o}^{(1)}\left\{\int_{0}^{\bar{\chi}} \mathrm{d} \tilde{\chi}\left[2\left(\Phi^{(1)}+\Psi^{(1)}\right)+(\bar{\chi}-\tilde{\chi})\left(\Phi^{(1) \prime}+\Psi^{(1) \prime}\right)\right]-\frac{1}{\mathcal{H}} \Delta \ln a^{(1)}\right\} \\
& +v_{\perp i o}^{(1)}\left\{-2 S_{\perp}^{i(1)}+\int_{0}^{\bar{\chi}} \mathrm{d} \tilde{\chi}\left[-2\left(1-2 \frac{\tilde{\chi}}{\bar{\chi}}\right) \tilde{\partial}_{\perp}^{i}\left(\Phi^{(1)}+\Psi^{(1)}\right)+\frac{\tilde{\chi}}{\bar{\chi}}(\bar{\chi}-\tilde{\chi}) \tilde{\partial}_{\perp}^{i}\left(\Phi^{(1) \prime}+\Psi^{(1) \prime}\right)\right]\right. \\
& \left.-\frac{1}{\mathcal{H}} \partial_{\perp}^{i} \Delta \ln a^{(1)}\right\},
\end{aligned}
$$

and

$$
\begin{aligned}
& \kappa_{\text {post-Born }}^{(2)}=\int_{0}^{\bar{\chi}} \mathrm{d} \tilde{\chi} \frac{\tilde{\chi}}{\bar{\chi}}\left\{\tilde{\nabla}_{\perp}^{2}\left(\Phi^{(1)}+\Psi^{(1)}\right) \delta x_{\|}^{(1)}+\partial_{\perp i}\left(\Phi^{(1)}+\Psi^{(1)}\right) \partial_{\perp}^{i} \delta x_{\|}^{(1)}+\frac{1}{\tilde{\chi}} \tilde{\partial}_{\perp i}\left(\Phi^{(1)}+\Psi^{(1)}\right) \delta x_{\perp}^{i(1)}\right. \\
& \left.-\frac{2}{\tilde{\chi}}\left(\Phi^{(1)}+\Psi^{(1)}\right) \kappa^{(1)}\right\}+\int_{0}^{\bar{\chi}} \mathrm{d} \tilde{\chi}(\bar{\chi}-\tilde{\chi}) \frac{\tilde{\chi}}{\bar{\chi}}\left\{-\left[\tilde{\nabla}_{\perp}^{2}\left(\Phi^{(1) \prime}+\Psi^{(1) \prime}\right)\right] T^{(1)}-\tilde{\partial}_{\perp}^{i}\left(\Phi^{(1) \prime}+\Psi^{(1) \prime}\right) \tilde{\partial}_{\perp i} T^{(1)}\right. \\
& +\left(\Phi^{(1)}-\Psi^{(1)}-2 I^{(1)}\right) \tilde{\nabla}_{\perp}^{2}\left(\Phi^{(1)}+\Psi^{(1)}\right)+\partial_{\perp i}\left(\Phi^{(1)}+\Psi^{(1)}\right)\left[\tilde{\partial}_{\perp}^{i}\left(\Phi^{(1)}-\Psi^{(1)}\right)-2 \tilde{\partial}_{\perp}^{i} I^{(1)}\right] \\
& +\frac{2}{\tilde{\chi}}\left[-\frac{1}{\tilde{\chi}}\left(\Phi^{(1)}+\Psi^{(1)}\right)+\frac{\mathrm{d}}{\mathrm{d} \tilde{\chi}}\left(\Phi^{(1)}+\Psi^{(1)}\right)\right] \kappa^{(1)}-\frac{2}{\tilde{\chi}} \tilde{\partial}_{\perp i}\left(\Phi^{(1)}+\Psi^{(1)}\right) S_{\perp}^{i(1)}-\frac{2}{\tilde{\chi}}\left(\Phi^{(1)}+\Psi^{(1)}\right) \tilde{\partial}_{\perp m} S_{\perp}^{m(1)} \\
& +\left[\tilde{\partial}_{\perp i} \tilde{\nabla}_{\perp}^{2}\left(\Phi^{(1)}+\Psi^{(1)}\right)+\frac{1}{\tilde{\chi}} \tilde{\partial}_{\perp i}\left(\Phi^{(1) \prime}+\Psi^{(1) \prime}\right)+\frac{2}{\tilde{\chi}^{2}} \tilde{\partial}_{\perp i}\left(\Phi^{(1)}+\Psi^{(1)}\right)\right] \delta x_{\perp}^{i(1)}-\left[\tilde{\partial}_{\perp}^{(j} \tilde{\partial}_{\perp}^{m)}\left(\Phi^{(1)}+\Psi^{(1)}\right)\right. \\
& \left.\left.+\frac{1}{\tilde{\chi}} n^{[j} \partial_{\perp}^{m]}\left(\Phi^{(1)}+\Psi^{(1)}\right)+\frac{1}{\tilde{\chi}} \mathcal{P}^{j m}\left(\Phi^{(1) \prime}+\Psi^{(1) \prime}\right)+\frac{1}{\tilde{\chi}^{2}} \mathcal{P}^{j m}\left(\Phi^{(1)}+\Psi^{(1)}\right)\right]\left(\gamma_{m j}^{(1)}+\mathcal{P}_{m j} \kappa^{(1)}+\theta_{m j}^{(1)}\right)\right\} \\
& -2\left(\Phi_{o}^{(1)}+\Psi_{o}^{(1)}\right) v_{\| o}^{(1)}-2 v_{\| o}^{(1)}\left(\Phi_{o}^{(1)}-v_{\| o}^{(1)}\right)-2 v_{\| o}^{(1)} \int_{0}^{\bar{\chi}} \mathrm{d} \tilde{\chi} \frac{(\bar{\chi}-\tilde{\chi})}{\bar{\chi} \tilde{\chi}}\left(\Phi^{(1)}+\Psi^{(1)}\right)-2\left(\Phi_{o}^{(1)}-v_{\| o}^{(1)}\right) \kappa^{(1)} \\
& +\frac{2}{\bar{\chi}} v_{\perp i o}^{(1)} \int_{0}^{\bar{\chi}} \mathrm{d} \tilde{\chi}(\bar{\chi}-\tilde{\chi}) \partial_{\perp}^{i}\left(\Phi^{(1)}+\Psi^{(1)}\right) .
\end{aligned}
$$

Finally, in order to obtain $\Delta_{g}^{(2)}$ [see Eq. 77$]$ ], we use

$$
\begin{gathered}
\frac{1}{2} \hat{g}_{\mu}^{\mu(2)}-\frac{1}{2} \hat{g}_{\mu}^{\nu(1)} \hat{g}_{\nu}^{\mu(1)}=\Phi^{(2)}-3 \Psi^{(2)}-2\left(\Phi^{(1)}\right)^{2}-6\left(\Psi^{(1)}\right)^{2} \\
E_{\hat{0}}^{0(2)}+E_{\hat{0}}^{\|(2)}=-\Phi^{(2)}+v_{\|}^{(2)}+3\left(\Phi^{(1)}\right)^{2}+v_{i}^{(1)} v^{i(1)} \\
\frac{1}{\mathcal{H}} \hat{g}_{\mu}^{\mu(1) \prime} \Delta \ln a^{(1)}+\left(\partial_{\|} \hat{g}_{\mu}^{\mu(1)}\right) \Delta x_{\|}^{(1)}=-\frac{2}{\mathcal{H}} \frac{\mathrm{d}}{\mathrm{d} \bar{\chi}}\left(\Phi^{(1)}-3 \Psi^{(1)}\right) \Delta \ln a^{(1)}-2 \partial_{\|}\left(\Phi^{(1)}-3 \Psi^{(1)}\right) T^{(1)} \\
\left(\partial_{\perp i} \hat{g}_{\mu}^{\mu(1)}\right) \Delta x_{\perp}^{i(1)}=-2 \bar{\chi} v_{\perp o}^{i(1)} \partial_{\perp i}\left(\Phi^{(1)}-3 \Psi^{(1)}\right)-2 \partial_{\perp i}\left(\Phi^{(1)}-3 \Psi^{(1)}\right) \int_{0}^{\bar{\chi}} \mathrm{d} \tilde{\chi}\left\{+(\bar{\chi}-\tilde{\chi})\left[\tilde{\partial}_{\perp}^{i}\left(\Phi^{(1)}+\Psi^{(1)}\right)\right]\right\} \\
\frac{2}{\mathcal{H}} \delta_{g}^{(1) \prime} \Delta \ln a^{(1)}+2\left(\partial_{\|} \delta_{g}^{(1)}\right) \Delta x_{\|}^{(1)}=-\frac{2}{\mathcal{H}} \frac{\mathrm{d}}{\mathrm{d} \bar{\chi}}\left(\delta_{g}^{(1)}\right) \Delta \ln a^{(1)}-2\left(\partial_{\|} \delta_{g}^{(1)}\right) T^{(1)}
\end{gathered}
$$




$$
\begin{aligned}
& \Delta x_{\perp i}^{(1)} \partial_{\perp}^{i} \delta_{g}^{(1)}=\delta x_{\perp i}^{(1)} \partial_{\perp}^{i} \delta_{g}^{(1)}=-\bar{\chi} v_{\perp o}^{i(1)} \partial_{\perp i} \delta_{g}^{(1)}-\left(\partial_{\perp i} \delta_{g}^{(1)}\right) \int_{0}^{\bar{\chi}} \mathrm{d} \tilde{\chi}\left[(\bar{\chi}-\tilde{\chi}) \tilde{\partial}_{\perp}^{i}\left(\Phi^{(1)}+\Psi^{(1)}\right)\right] \\
& -\frac{2}{\bar{\chi}^{2}}\left(\Delta x_{\|}^{(1)}\right)^{2}=-\frac{2}{\bar{\chi}^{2}}\left(T^{(1)}\right)^{2}-\frac{2}{\bar{\chi}^{2} \mathcal{H}^{2}}\left(\Delta \ln a^{(1)}\right)^{2}-\frac{4}{\bar{\chi}^{2} \mathcal{H}} \Delta \ln a^{(1)} T^{(1)}, \\
& \frac{4}{\bar{\chi}} \Delta x_{\|}^{(1)} \kappa^{(1)}=-\frac{4}{\bar{\chi} \mathcal{H}} \Delta \ln a^{(1)} \kappa^{(1)}-\frac{4}{\bar{\chi}} T^{(1)} \kappa^{(1)} \\
& -\left(\partial_{\perp j} \Delta x_{\perp}^{i(1)}\right)\left(\partial_{\perp i} \Delta x_{\perp}^{j(1)}\right)=-2\left(v_{\| o}^{(1)}\right)^{2}-2\left(\Phi_{o}^{(1)}\right)^{2}+4 v_{\| o}^{(1)} \Psi_{o}^{(1)}-2\left(\Psi_{o}^{(1)}\right)^{2}+4 \Phi_{o}^{(1)} v_{\| o}^{(1)}-4 \Phi_{o}^{(1)} \Psi_{o}^{(1)} \\
& +2\left(\Phi_{o}^{(1)}-v_{\| o}^{(1)}+\Psi_{o}^{(1)}\right)\left\{2\left(\Phi^{(1)}+\Psi^{(1)}-2 I^{(1)}\right)-\int_{0}^{\bar{\chi}} \mathrm{d} \tilde{\chi}\left[\frac{\tilde{\chi}}{\bar{\chi}}\left(2 \tilde{\partial}_{\|}+(\bar{\chi}-\tilde{\chi}) \mathcal{P}^{m n} \tilde{\partial}_{m} \tilde{\partial}_{n}\right)\left(\Phi^{(1)}+\Psi^{(1)}\right)\right]\right\} \\
& -2\left(\Phi^{(1)}+\Psi^{(1)}-2 I^{(1)}\right)^{2}+2\left(\Phi^{(1)}+\Psi^{(1)}-2 I^{(1)}\right) \int_{0}^{\bar{\chi}} \mathrm{d} \tilde{\chi}\left[\frac{\tilde{\chi}}{\bar{\chi}}\left(2 \tilde{\partial}_{\|}+(\bar{\chi}-\tilde{\chi}) \mathcal{P}^{m n} \tilde{\partial}_{m} \tilde{\partial}_{n}\right)\left(\Phi^{(1)}+\Psi^{(1)}\right)\right] \\
& -\left[\int_{0}^{\bar{\chi}} \mathrm{d} \tilde{\chi} \frac{\tilde{\chi}}{\bar{\chi}}\left(\mathcal{P}_{j}^{i} \tilde{\partial}_{\|}+(\bar{\chi}-\tilde{\chi}) \mathcal{P}_{j}^{p} \mathcal{P}^{i q} \tilde{\partial}_{q} \tilde{\partial}_{p}\right)\left(\Phi^{(1)}+\Psi^{(1)}\right)\right] \\
& \times\left[\int_{0}^{\bar{\chi}} \mathrm{d} \tilde{\chi} \frac{\tilde{\chi}}{\bar{\chi}}\left(\mathcal{P}_{i}^{j} \tilde{\partial}_{\|}+(\bar{\chi}-\tilde{\chi}) \mathcal{P}_{i}^{n} \mathcal{P}^{j m} \tilde{\partial}_{m} \tilde{\partial}_{n}\right)\left(\Phi^{(1)}+\Psi^{(1)}\right)\right], \\
& 2\left(\frac{1}{\bar{\chi}} \Delta x_{\perp i}^{(1)}-\partial_{\perp i} \Delta x_{\|}^{(1)}\right) \partial_{\|} \Delta x_{\perp}^{i(1)}=2 v_{\perp i o}^{(1)} v_{\perp o}^{i(1)}-2 v_{\perp i o}^{(1)}\left\{+2 S_{\perp}^{i(1)}+\partial_{\perp i}\left(\frac{1}{\mathcal{H}} \Delta \ln a^{(1)}+T^{(1)}\right)\right. \\
& \left.-\frac{1}{\bar{\chi}} \int_{0}^{\bar{\chi}} \mathrm{d} \tilde{\chi}\left[(\bar{\chi}-\tilde{\chi}) \tilde{\partial}_{\perp}^{i}\left(\Phi^{(1)}+\Psi^{(1)}\right)\right]\right\}+4 S_{\perp}^{i(1)}\left\{-\frac{1}{\bar{\chi}} \int_{0}^{\bar{\chi}} \mathrm{d} \tilde{\chi}\left[(\bar{\chi}-\tilde{\chi}) \tilde{\partial}_{\perp}^{i}\left(\Phi^{(1)}+\Psi^{(1)}\right)\right]\right. \\
& \left.+\partial_{\perp i}\left(\frac{1}{\mathcal{H}} \Delta \ln a^{(1)}+T^{(1)}\right)\right\} \\
& \frac{2}{\mathcal{H}}\left(E_{\hat{0}}^{0(1)}+E_{\hat{0}}^{\|(1)}\right)^{\prime} \Delta \ln a^{(1)}+2 \partial_{\|}\left(E_{\hat{0}}^{0(1)}+E_{\hat{0}}^{\|(1)}\right) \Delta x_{\|}^{(1)}=\frac{2}{\mathcal{H}} \frac{\mathrm{d}}{\mathrm{d} \bar{\chi}}\left(\Phi^{(1)}-v_{\|}^{(1)}\right) \Delta \ln a^{(1)}+2 \partial_{\|}\left(\Phi^{(1)}-v_{\|}^{(1)}\right) T^{(1)} \\
& \partial_{\perp i}\left(E_{\hat{0}}^{0(1)}+E_{\hat{0}}^{\|(1)}\right) \Delta x_{\perp}^{i(1)}=\bar{\chi} v_{\perp o}^{i(1)} \partial_{\perp i}\left(\Phi^{(1)}-v_{\|}^{(1)}\right)+\partial_{\perp i}\left(\Phi^{(1)}-v_{\|}^{(1)}\right) \int_{0}^{\bar{\chi}} \mathrm{d} \tilde{\chi}\left[(\bar{\chi}-\tilde{\chi}) \tilde{\partial}_{\perp}^{i}\left(\Phi^{(1)}+\Psi^{(1)}\right)\right] \\
& -2\left(\delta n_{\|}^{(1)}+\delta \nu^{(1)}\right) E_{\hat{0}}^{\|(1)}=-2 v_{\|}^{(1)}\left(\Phi^{(1)}+\Psi^{(1)}\right) \\
& -2 E_{\hat{0}}^{\perp i(1)} \partial_{\perp i}\left(\Delta x^{0(1)}+\Delta x_{\|}^{(1)}\right)=+2 v_{\perp}^{i(1)} \partial_{\perp i} T^{(1)}, \\
& -\left(\partial_{\|} \Delta x_{\|}^{(1)}\right)^{2}=-\left\{\left(\Phi^{(1)}+\Psi^{(1)}\right)+\frac{1}{\mathcal{H}}\left[\frac{\mathrm{d}}{\mathrm{d} \bar{\chi}}\left(\Phi^{(1)}-v_{\|}^{(1)}\right)+\left(\Phi^{(1) \prime}+\Psi^{(1) \prime}\right)\right]-\frac{\mathcal{H}^{\prime}}{\mathcal{H}^{2}} \Delta \ln a^{(1)}\right\}^{2} \\
& =-\left(\Phi^{(1)}+\Psi^{(1)}\right)^{2}-\frac{1}{\mathcal{H}^{2}}\left[\frac{\mathrm{d}}{\mathrm{d} \bar{\chi}}\left(\Phi^{(1)}-v_{\|}^{(1)}\right)+\left(\Phi^{(1) \prime}+\Psi^{(1) \prime}\right)\right]^{2}-\left(\frac{\mathcal{H}^{\prime}}{\mathcal{H}^{2}}\right)^{2}\left(\Delta \ln a^{(1)}\right)^{2} \\
& -\frac{2}{\mathcal{H}}\left(\Phi^{(1)}+\Psi^{(1)}\right)\left[\frac{\mathrm{d}}{\mathrm{d} \bar{\chi}}\left(\Phi^{(1)}-v_{\|}^{(1)}\right)+\left(\Phi^{(1) \prime}+\Psi^{(1) \prime}\right)\right]+2 \frac{\mathcal{H}^{\prime}}{\mathcal{H}^{2}}\left(\Phi^{(1)}+\Psi^{(1)}\right) \Delta \ln a^{(1)} \\
& +2 \frac{\mathcal{H}^{\prime}}{\mathcal{H}^{3}}\left[\frac{\mathrm{d}}{\mathrm{d} \bar{\chi}}\left(\Phi^{(1)}-v_{\|}^{(1)}\right)+\left(\Phi^{(1) \prime}+\Psi^{(1) \prime}\right)\right] \Delta \ln a^{(1)}
\end{aligned}
$$




$$
-\left(E_{\hat{0}}^{0(1)}+E_{\hat{0}}^{\|(1)}\right)^{2}=-\left(\Phi^{(1)}-v_{\|}^{(1)}\right)^{2} .
$$

Using $\Delta \ln a^{(2)}$ in Eq. $202, \Delta x_{\|}^{(2)}$ in Eq. $212, \partial_{\|} \Delta x_{\|}^{(2)}$ in Eq. 215 and $\kappa^{(2)}$ in Eq. 216 , we finally obtain in Poisson gauge the number density fluctuations at second order:

$$
\begin{aligned}
& \Delta_{g}^{(2)}=\delta_{g}^{(2)}+b_{e} \Delta \ln a^{(2)}+\partial_{\|} \Delta x_{\|}^{(2)}+\frac{2}{\bar{\chi}} \Delta x_{\|}^{(2)}-2 \kappa^{(2)}+v_{\|}^{(2)}-3 \Psi^{(2)}+\left(\Delta_{g}^{(1)}\right)^{2} \\
& -\left(\delta_{g}^{(1)}\right)^{2}-3\left(\Phi^{(1)}\right)^{2}-9\left(\Psi^{(1)}\right)^{2}+v_{\perp i}^{(1)} v_{\perp}^{i(1)}-2 v_{\|}^{(1)} \Psi^{(1)}-6 \Phi^{(1)} \Psi^{(1)}+8 \Phi^{(1)} I^{(1)}+8 \Psi^{(1)} I^{(1)}-8\left(I^{(1)}\right)^{2} \\
& -\frac{1}{\mathcal{H}^{2}}\left[\frac{\mathrm{d}}{\mathrm{d} \bar{\chi}}\left(\Phi^{(1)}-v_{\|}^{(1)}\right)+\left(\Phi^{(1) \prime}+\Psi^{(1) \prime}\right)\right]^{2}-\frac{2}{\mathcal{H}}\left(\Phi^{(1)}+\Psi^{(1)}\right)\left[\frac{\mathrm{d}}{\mathrm{d} \bar{\chi}}\left(\Phi^{(1)}-v_{\|}^{(1)}\right)+\left(\Phi^{(1) \prime}+\Psi^{(1) \prime}\right)\right] \\
& +\left\{-\frac{2}{\mathcal{H}} \frac{\mathrm{d}}{\mathrm{d} \bar{\chi}}\left(v_{\|}^{(1)}-3 \Psi^{(1)}+\delta_{g}^{(1)}\right)+2 \frac{\mathcal{H}^{\prime}}{\mathcal{H}^{2}}\left(\Phi^{(1)}+\Psi^{(1)}\right)+2 \frac{\mathcal{H}^{\prime}}{\mathcal{H}^{3}}\left[\frac{\mathrm{d}}{\mathrm{d} \bar{\chi}}\left(\Phi^{(1)}-v_{\|}^{(1)}\right)+\left(\Phi^{(1) \prime}+\Psi^{(1) \prime}\right)\right]\right. \\
& \left.-\frac{4}{\bar{\chi} \mathcal{H}} \kappa^{(1)}\right\} \Delta \ln a^{(1)}+\left[-b_{e}+\frac{\mathrm{d} \ln b_{e}}{\mathrm{~d} \ln \bar{a}}-\left(\frac{\mathcal{H}^{\prime}}{\mathcal{H}^{2}}\right)^{2}-\frac{2}{\bar{\chi}^{2} \mathcal{H}^{2}}\right]\left(\Delta \ln a^{(1)}\right)^{2}-\frac{4}{\bar{\chi}^{2} \mathcal{H}} \Delta \ln a^{(1)} T^{(1)}+2 v_{\perp}^{i(1)} \partial_{\perp i} T^{(1)} \\
& -2 \partial_{\|}\left(v_{\|}^{(1)}-3 \Psi^{(1)}+\delta_{g}^{(1)}\right) T^{(1)}-\frac{4}{\bar{\chi}} T^{(1)} \kappa^{(1)}-\frac{2}{\bar{\chi}^{2}}\left(T^{(1)}\right)^{2}+4 \partial_{\perp i}\left(\frac{1}{\mathcal{H}} \Delta \ln a^{(1)}+T^{(1)}\right) S_{\perp}^{i(1)} \\
& +2\left(\Phi^{(1)}+\Psi^{(1)}-2 I^{(1)}\right) \int_{0}^{\bar{\chi}} \mathrm{d} \tilde{\chi}\left[\frac{\tilde{\chi}}{\bar{\chi}}\left(2 \tilde{\partial}_{\|}+(\bar{\chi}-\tilde{\chi}) \mathcal{P}^{m n} \tilde{\partial}_{m} \tilde{\partial}_{n}\right)\left(\Phi^{(1)}+\Psi^{(1)}\right)\right] \\
& -2 \partial_{\perp i}\left(v_{\|}^{(1)}-3 \Psi^{(1)}+\delta_{g}^{(1)}\right) \int_{0}^{\bar{\chi}} \mathrm{d} \tilde{\chi}(\bar{\chi}-\tilde{\chi}) \tilde{\partial}_{\perp}^{i}\left(\Phi^{(1)}+\Psi^{(1)}\right)-\frac{4}{\bar{\chi}} S_{\perp}^{i(1)} \int_{0}^{\bar{\chi}} \mathrm{d} \tilde{\chi}\left[(\bar{\chi}-\tilde{\chi}) \tilde{\partial}_{\perp}^{i}\left(\Phi^{(1)}+\Psi^{(1)}\right)\right] \\
& -\left[\int_{0}^{\bar{\chi}} \mathrm{d} \tilde{\chi} \frac{\tilde{\chi}}{\bar{\chi}}\left(\mathcal{P}_{j}^{i} \tilde{\partial}_{\|}+(\bar{\chi}-\tilde{\chi}) \mathcal{P}_{j}^{p} \mathcal{P}^{i q} \tilde{\partial}_{q} \tilde{\partial}_{p}\right)\left(\Phi^{(1)}+\Psi^{(1)}\right)\right] \\
& \times\left[\int_{0}^{\bar{\chi}} \mathrm{d} \tilde{\chi} \frac{\tilde{\chi}}{\bar{\chi}}\left(\mathcal{P}_{i}^{j} \tilde{\partial}_{\|}+(\bar{\chi}-\tilde{\chi}) \mathcal{P}_{i}^{n} \mathcal{P}^{j m} \tilde{\partial}_{m} \tilde{\partial}_{n}\right)\left(\Phi^{(1)}+\Psi^{(1)}\right)\right] \\
& -2\left(v_{\| o}^{(1)}\right)^{2}-2\left(\Phi_{o}^{(1)}\right)^{2}+4 v_{\| o}^{(1)} \Psi_{o}^{(1)}-2\left(\Psi_{o}^{(1)}\right)^{2}+4 \Phi_{o}^{(1)} v_{\| o}^{(1)}-4 \Phi_{o}^{(1)} \Psi_{o}^{(1)}+2 v_{\perp i o}^{(1)} v_{\perp o}^{i(1)} \\
& +2\left(\Phi_{o}^{(1)}-v_{\| o}^{(1)}+\Psi_{o}^{(1)}\right)\left\{2\left(\Phi^{(1)}+\Psi^{(1)}-2 I^{(1)}\right)-\int_{0}^{\bar{\chi}} \mathrm{d} \tilde{\chi}\left[\frac{\tilde{\chi}}{\bar{\chi}}\left(2 \tilde{\partial}_{\|}+(\bar{\chi}-\tilde{\chi}) \mathcal{P}^{m n} \tilde{\partial}_{m} \tilde{\partial}_{n}\right)\left(\Phi^{(1)}+\Psi^{(1)}\right)\right]\right\} \\
& -2 v_{\perp i o}^{(1)}\left\{\bar{\chi} \partial_{\perp}^{i}\left(v_{\|}^{(1)}-3 \Psi^{(1)}+\delta_{g}^{(1)}\right)+2 S_{\perp}^{i(1)}+\partial_{\perp i}\left(\frac{1}{\mathcal{H}} \Delta \ln a^{(1)}+T^{(1)}\right)\right. \\
& \left.-\frac{1}{\bar{\chi}} \int_{0}^{\bar{\chi}} \mathrm{d} \tilde{\chi}\left[(\bar{\chi}-\tilde{\chi}) \tilde{\partial}_{\perp}^{i}\left(\Phi^{(1)}+\Psi^{(1)}\right)\right]\right\} \text {. }
\end{aligned}
$$

This is the main result in Poisson gauge. If we explicitly identify the weak lensing shear and rotation contributions, 
it becomes

$$
\begin{aligned}
\Delta_{g}^{(2)}= & \delta_{g}^{(2)}+b_{e} \Delta \ln a^{(2)}+\partial_{\|} \Delta x_{\|}^{(2)}+\frac{2}{\bar{\chi}} \Delta x_{\|}^{(2)}-2 \kappa^{(2)}+v_{\|}^{(2)}-3 \Psi^{(2)}+\left(\Delta_{g}^{(1)}\right)^{2}-\left(\delta_{g}^{(1)}\right)^{2}-\left(\Phi^{(1)}\right)^{2}-7\left(\Psi^{(1)}\right)^{2} \\
& +v_{\perp i}^{(1)} v_{\perp}^{i(1)}-2 v_{\|}^{(1)} \Psi^{(1)}-2 \Phi^{(1)} \Psi^{(1)}-2\left(\kappa^{(1)}\right)^{2}-2\left|\gamma^{(1)}\right|^{2}+\vartheta_{i j}^{(1)} \vartheta^{i j(1)} \\
& -\frac{1}{\mathcal{H}^{2}}\left[\frac{\mathrm{d}}{\mathrm{d} \bar{\chi}}\left(\Phi^{(1)}-v_{\|}^{(1)}\right)+\left(\Phi^{(1) \prime}+\Psi^{(1) \prime}\right)\right]^{2}-\frac{2}{\mathcal{H}}\left(\Phi^{(1)}+\Psi^{(1)}\right)\left[\frac{\mathrm{d}}{\mathrm{d} \bar{\chi}}\left(\Phi^{(1)}-v_{\|}^{(1)}\right)+\left(\Phi^{(1) \prime}+\Psi^{(1) \prime}\right)\right] \\
& +\left\{-\frac{2}{\mathcal{H}} \frac{\mathrm{d}}{\mathrm{d} \bar{\chi}}\left(v_{\|}^{(1)}-3 \Psi^{(1)}+\delta_{g}^{(1)}\right)+2 \frac{\mathcal{H}^{\prime}}{\mathcal{H}^{2}}\left(\Phi^{(1)}+\Psi^{(1)}\right)+2 \frac{\mathcal{H}^{\prime}}{\mathcal{H}^{3}}\left[\frac{\mathrm{d}}{\mathrm{d} \bar{\chi}}\left(\Phi^{(1)}-v_{\|}^{(1)}\right)+\left(\Phi^{(1) \prime}+\Psi^{(1) \prime}\right)\right]\right. \\
& \left.-\frac{4}{\bar{\chi} \mathcal{H}} \kappa^{(1)}\right\} \Delta \ln a^{(1)}+\left[-b_{e}+\frac{\mathrm{d} \ln b_{e}}{\mathrm{~d} \ln \bar{a}}-\left(\frac{\mathcal{H}^{\prime}}{\mathcal{H}^{2}}\right)^{2}-\frac{2}{\bar{\chi}^{2} \mathcal{H}^{2}}\right]\left(\Delta \ln a^{(1)}\right)^{2}-\frac{4}{\bar{\chi}^{2} \mathcal{H}} \Delta \ln a^{(1)} T^{(1)}+2 v_{\perp}^{i(1)} \partial_{\perp i} T^{(1)} \\
& -2 \partial_{\|}\left(v_{\|}^{(1)}-3 \Psi^{(1)}+\delta_{g}^{(1)}\right) T^{(1)}-\frac{4}{\bar{\chi}} T^{(1)} \kappa^{(1)}-\frac{2}{\bar{\chi}^{2}}\left(T^{(1)}\right)^{2}+4 \partial_{\perp i}\left(\frac{1}{\mathcal{H}} \Delta \ln a^{(1)}+T^{(1)}\right) S_{\perp}^{i(1)} \\
& -2 \partial_{\perp i}\left(v_{\|}^{(1)}-3 \Psi^{(1)}+\delta_{g}^{(1)}\right) \int_{0}^{\bar{\chi}} \mathrm{d} \tilde{\chi}(\bar{\chi}-\tilde{\chi}) \tilde{\partial}_{\perp}^{i}\left(\Phi^{(1)}+\Psi^{(1)}\right)-\frac{4}{\bar{\chi}} S_{\perp}^{i(1)} \int_{0}^{\bar{\chi}} \mathrm{d} \tilde{\chi}\left[(\bar{\chi}-\tilde{\chi}) \tilde{\partial}_{\perp}^{i}\left(\Phi^{(1)}+\Psi^{(1)}\right)\right] \\
& +2 v_{\perp i o}^{(1)} v_{\perp o}^{i(1)}-2 v_{\perp i o}^{(1)}\left\{\bar{\chi} \partial_{\perp}^{i}\left(v_{\|}^{(1)}-3 \Psi^{(1)}+\delta_{g}^{(1)}\right)+2 S_{\perp}^{i(1)}+\partial_{\perp i}\left(\frac{1}{\mathcal{H}} \Delta \ln a^{(1)}+T^{(1)}\right)\right. \\
& \left.-\frac{1}{\bar{\chi}} \int_{0}^{\bar{\chi}} \mathrm{d} \tilde{\chi}\left[(\bar{\chi}-\tilde{\chi}) \tilde{\partial}_{\perp}^{i}\left(\Phi^{(1)}+\Psi^{(1)}\right)\right]\right\},
\end{aligned}
$$

where $\gamma_{i j}^{(1)}$ and $\vartheta_{i j}^{(1)} \vartheta^{i j(1)}$ are given in Eqs. B10 and B11.

\section{Assuming no velocity bias}

At first order, assuming galaxy velocities follow the matter velocity field, then the comoving velocities are the same and we can write

$$
\partial_{\|} \Delta x_{\|}^{(1)}=\Phi^{(1)}-v_{\|}^{(1)}+\Psi^{(1)}-\frac{1}{\mathcal{H}} \partial_{\|} v_{\|}^{(1)}+\frac{1}{\mathcal{H}} \Psi^{(1) \prime}+\frac{\bar{a}^{2}}{\mathcal{H} \bar{\rho}_{m}}\left(\mathcal{E}_{m}^{\|(1)}-\mathcal{E}_{m}^{0(0)} v_{\|}^{(1)}\right)-\frac{\mathcal{H}^{\prime}}{\mathcal{H}^{2}} \Delta \ln a^{(1)} .
$$

Then

$$
\begin{aligned}
\Delta_{g}^{(1)} & =\delta_{g}^{(1)}+\left(b_{e}-\frac{\mathcal{H}^{\prime}}{\mathcal{H}^{2}}-\frac{2}{\bar{\chi} \mathcal{H}}\right) \Delta \ln a^{(1)}-\frac{1}{\mathcal{H}} \partial_{\|} v_{\|}^{(1)}+\frac{1}{\mathcal{H}} \Psi^{(1) \prime}+\Phi^{(1)}-2 \Psi^{(1)}-b_{m} v_{\|}^{(1)}-\frac{2}{\bar{\chi}} T^{(1)}-2 \kappa^{(1)} \\
& +\frac{a^{2}}{\mathcal{H} \bar{\rho}_{m}} \mathcal{E}_{m}^{\|(1)} .
\end{aligned}
$$


At second order, through Eq. $\mathrm{B} 8 \mathrm{r}$ we have

$$
\begin{aligned}
& \Delta \ln a^{(2)}=+\Phi_{o}^{(2)}-v_{\| o}^{(2)}-\left(\Phi_{o}^{(1)}\right)^{2}-6 \Phi_{o}^{(1)} v_{\| o}^{(1)}+v_{k o}^{(1)} v_{o}^{k(1)}+2 \Psi_{o}^{(1)} v_{\| o}^{(1)}+2\left(\Phi_{o}^{(1)}-v_{\| o}^{(1)}\right)\left[-3 \Phi^{(1)}-\frac{1}{\mathcal{H}} \partial_{\|} v_{\|}^{(1)}\right. \\
& \left.+\frac{1}{\mathcal{H}} \Psi^{(1) \prime}-b_{m} v_{\|}^{(1)}+2 \bar{\chi} \partial_{\|} \Phi^{(1)}-\bar{\chi}\left(\Phi^{(1) \prime}-\Psi^{(1) \prime}\right)+\frac{a^{2}}{\mathcal{H} \bar{\rho}_{m}} \mathcal{E}_{m}^{\|(1)}+4 I^{(1)}\right]-2 v_{\perp o}^{i(1)}\left[\bar{\chi} \partial_{\perp i}\left(\Phi^{(1)}+v_{\|}^{(1)}\right)\right. \\
& \left.-2 \int_{0}^{\bar{\chi}} \mathrm{d} \tilde{\chi} \tilde{\partial}_{\perp i} \Phi^{(1)}\right]-\Phi^{(2)}+v_{\|}^{(2)}+7\left(\Phi^{(1)}\right)^{2}-\left(1+2 b_{m}\right)\left(v_{\|}^{(1)}\right)^{2}+v_{\perp i}^{(1)} v_{\perp}^{i(1)}-2 v_{\|}^{(1)} \Psi^{(1)}+2 b_{m} \Phi^{(1)} v_{\|}^{(1)} \\
& -\frac{2}{\mathcal{H}}\left(\Phi^{(1)}-v_{\|}^{(1)}\right)\left(\Psi^{(1) \prime}-\partial_{\|} v_{\|}^{(1)}+\frac{a^{2}}{\bar{\rho}_{m}} \mathcal{E}_{m}^{\|(1)}\right)-4\left[3 \Phi^{(1)}+\frac{1}{\mathcal{H}} \partial_{\|} v_{\|}^{(1)}-\frac{1}{\mathcal{H}} \Psi^{(1) \prime}+b_{m} v_{\|}^{(1)}\right. \\
& \left.-\frac{\bar{a}^{2}}{\mathcal{H} \bar{\rho}_{m}} \mathcal{E}_{m}^{\|(1)}\right] I^{(1)}+4 v_{\perp i}^{(1)} S_{\perp}^{i(1)}-2 \partial_{\|}\left(\Phi^{(1)}+v_{\|}^{(1)}\right) T^{(1)}-2\left[2 \partial_{\|} \Phi^{(1)}-\left(\Phi^{(1) \prime}-\Psi^{(1) \prime}\right)\right] \\
& \times \int_{0}^{\bar{\chi}} \mathrm{d} \tilde{\chi}\left[2 \Phi^{(1)}+(\bar{\chi}-\tilde{\chi})\left(\Phi^{(1) \prime}+\Psi^{(1) \prime}\right)\right]-2\left[\partial_{\perp i}\left(\Phi^{(1)}+v_{\|}^{(1)}\right)-\frac{1}{\bar{\chi}} v_{\perp i}^{(1)}\right] \\
& \times \int_{0}^{\bar{\chi}} \mathrm{d} \tilde{\chi}\left[(\bar{\chi}-\tilde{\chi}) \tilde{\partial}_{\perp}^{i}\left(\Phi^{(1)}+\Psi^{(1)}\right)\right]+2 I^{(2)}-4 \int_{0}^{\bar{\chi}} \mathrm{d} \tilde{\chi}\left[\left(\Psi^{(1)}+2 I^{(1)}\right)\left(\Phi^{(1) \prime}+\Psi^{(1) \prime}\right)\right. \\
& \left.+\left(\Phi^{(1)}+\Psi^{(1)}\right) \frac{\mathrm{d}}{\mathrm{d} \tilde{\chi}} \Phi^{(1)}+2 S_{\perp}^{i(1)} \tilde{\partial}_{\perp i} \Phi^{(1)}\right]+\Delta \ln a_{\text {post-Born }}^{(2)}, \\
& \Delta x^{0(2)}=+\frac{1}{\mathcal{H}} \Phi_{o}^{(2)}-\frac{1}{\mathcal{H}} v_{\| o}^{(2)}-\left(\frac{\mathcal{H}^{\prime}}{\mathcal{H}^{3}}+\frac{2}{\mathcal{H}}\right)\left(\Phi_{o}^{(1)}\right)^{2}+2\left(\frac{\mathcal{H}^{\prime}}{\mathcal{H}^{3}}-\frac{2}{\mathcal{H}}\right) \Phi_{o}^{(1)} v_{\| o}^{(1)}-\frac{\mathcal{H}^{\prime}}{\mathcal{H}^{3}}\left(v_{\| o}^{(1)}\right)^{2}+\frac{1}{\mathcal{H}} v_{\perp i o}^{(1)} v_{\perp o}^{i(1)} \\
& +\frac{2}{\mathcal{H}} \Psi_{o}^{(1)} v_{\| o}^{(1)}+2\left(\Phi_{o}^{(1)}-v_{\| o}^{(1)}\right)\left\{\left(\frac{\mathcal{H}^{\prime}}{\mathcal{H}^{3}}-\frac{2}{\mathcal{H}}\right) \Phi^{(1)}-\left[\frac{\mathcal{H}^{\prime}}{\mathcal{H}^{3}}+\frac{1}{\mathcal{H}}\left(1+b_{m}\right)\right] v_{\|}^{(1)}-\frac{\bar{\chi}}{\mathcal{H}} \Phi^{(1) \prime}\right. \\
& \left.-\frac{1}{\mathcal{H}^{2}}\left(\partial_{\|} v_{\|}^{(1)}-\frac{\bar{a}^{2}}{\bar{\rho}_{m}} \mathcal{E}^{\|(1)}\right)+\left(\frac{\bar{\chi}}{\mathcal{H}}+\frac{1}{\mathcal{H}^{2}}\right) \Psi^{(1) \prime}+2 \frac{\bar{\chi}}{\mathcal{H}} \partial_{\|} \Phi^{(1)}-2\left(\frac{\mathcal{H}^{\prime}}{\mathcal{H}^{3}}-\frac{1}{\mathcal{H}}\right) I^{(1)}\right\} \\
& -2 v_{\perp o}^{i(1)}\left[\frac{\bar{\chi}}{\mathcal{H}} \partial_{\perp i}\left(\Phi^{(1)}+v_{\|}^{(1)}\right)-\frac{2}{\mathcal{H}} \int_{0}^{\bar{\chi}} \mathrm{d} \tilde{\chi} \tilde{\partial}_{\perp i} \Phi^{(1)}\right]-\frac{1}{\mathcal{H}} \Phi^{(2)}+\frac{1}{\mathcal{H}} v_{\|}^{(2)}+\left(-\frac{\mathcal{H}^{\prime}}{\mathcal{H}^{3}}+\frac{6}{\mathcal{H}}\right)\left(\Phi^{(1)}\right)^{2} \\
& -\left[\frac{\mathcal{H}^{\prime}}{\mathcal{H}^{3}}+\frac{2}{\mathcal{H}}\left(1+b_{m}\right)\right]\left(v_{\|}^{(1)}\right)^{2}+\frac{1}{\mathcal{H}} v_{\perp i}^{(1)} v_{\perp}^{i(1)}-\frac{2}{\mathcal{H}} v_{\|}^{(1)} \Psi^{(1)}+2\left[\frac{\mathcal{H}^{\prime}}{\mathcal{H}^{3}}+\frac{1}{\mathcal{H}}\left(1+b_{m}\right)\right] \Phi^{(1)} v_{\|}^{(1)} \\
& +\frac{2}{\mathcal{H}^{2}}\left(\Phi^{(1)}-v_{\|}^{(1)}\right)\left(-\Psi^{(1) \prime}+\partial_{\|} v_{\|}^{(1)}-\frac{\bar{a}^{2}}{\bar{\rho}_{m}} \mathcal{E}_{m}^{\|(1)}\right)+4\left\{\left(\frac{\mathcal{H}^{\prime}}{\mathcal{H}^{3}}-\frac{2}{\mathcal{H}}\right) \Phi^{(1)}-\left[\frac{\mathcal{H}^{\prime}}{\mathcal{H}^{3}}+\frac{1}{\mathcal{H}}\left(1+b_{m}\right)\right] v_{\|}^{(1)}\right. \\
& \left.-\frac{1}{\mathcal{H}^{2}}\left(-\Psi^{(1) \prime}+\partial_{\|} v_{\|}^{(1)}-\frac{\bar{a}^{2}}{\bar{\rho}_{m}} \mathcal{E}_{m}^{\|(1)}\right)-\left(\frac{\mathcal{H}^{\prime}}{\mathcal{H}^{3}}+\frac{1}{\mathcal{H}}\right) I^{(1)}\right\} I^{(1)}+\frac{4}{\mathcal{H}} v_{\perp i}^{(1)} S_{\perp}^{i(1)}-\frac{2}{\mathcal{H}} \partial_{\|}\left(\Phi^{(1)}+v_{\|}^{(1)}\right) T^{(1)} \\
& -\frac{2}{\mathcal{H}}\left[2 \partial_{\|} \Phi^{(1)}-\left(\Phi^{(1) \prime}-\Psi^{(1) \prime}\right)\right] \int_{0}^{\bar{\chi}} \mathrm{d} \tilde{\chi}\left[2 \Phi^{(1)}+(\bar{\chi}-\tilde{\chi})\left(\Phi^{(1) \prime}+\Psi^{(1) \prime}\right)\right]-\frac{2}{\mathcal{H}}\left[\partial_{\perp i}\left(\Phi^{(1)}+v_{\|}^{(1)}\right)\right. \\
& \left.-\frac{1}{\bar{\chi}} v_{\perp i}^{(1)}\right] \int_{0}^{\bar{\chi}} \mathrm{d} \tilde{\chi}\left[(\bar{\chi}-\tilde{\chi}) \tilde{\partial}_{\perp}^{i}\left(\Phi^{(1)}+\Psi^{(1)}\right)\right]+\frac{2}{\mathcal{H}} I^{(2)}-\frac{4}{\mathcal{H}} \int_{0}^{\bar{\chi}} \mathrm{d} \tilde{\chi}\left[\left(\Psi^{(1)}+2 I^{(1)}\right)\left(\Phi^{(1) \prime}+\Psi^{(1) \prime}\right)\right. \\
& \left.+\left(\Phi^{(1)}+\Psi^{(1)}\right) \frac{\mathrm{d}}{\mathrm{d} \tilde{\chi}} \Phi^{(1)}+2 S_{\perp}^{i(1)} \tilde{\partial}_{\perp i} \Phi^{(1)}\right]+\Delta x_{\text {post-Born }}^{0(2)},
\end{aligned}
$$




$$
\begin{aligned}
& \partial_{\|} \Delta x_{\|}^{(2)}=\left(\Phi_{o}^{(1)}\right)^{2}+2 \Phi_{o}^{(1)} v_{\| o}^{(1)}+2 v_{\| o}^{(1)} \Psi_{o}^{(1)}-\left(\Psi_{o}^{(1)}\right)^{2}-v_{\perp k o}^{(1)} v_{\perp o}^{k(1)}+2\left(\Phi_{o}^{(1)}-v_{\| o}^{(1)}\right) \\
& \times\left\{\left(-\frac{\mathcal{H}^{\prime}}{\mathcal{H}^{3}}+\frac{1}{\mathcal{H}}\right)\left[\frac{\bar{a}^{2}}{\bar{\rho}_{m}} \mathcal{E}_{m}^{\|(1)}-\partial_{\|} v_{\|}^{(1)}-\mathcal{H}\left(b_{m}+1\right) v_{\|}^{(1)}+\Psi^{(1) \prime}\right]-\frac{\bar{\chi}}{\mathcal{H}} \frac{\mathrm{d}}{\mathrm{d} \bar{\chi}}\left[2 \partial_{\|} \Phi^{(1)}-\left(\Phi^{(1) \prime}-\Psi^{(1) \prime}\right)\right]\right. \\
& \left.+\left(\Phi^{(1)}+\Psi^{(1)}\right)-\bar{\chi} \frac{\mathrm{d}}{\mathrm{d} \bar{\chi}}\left(\Phi^{(1)}+\Psi^{(1)}\right)-\frac{1}{\mathcal{H}^{2}} \frac{\mathrm{d}}{\mathrm{d} \bar{\chi}}\left[\frac{\bar{a}^{2}}{\bar{\rho}_{m}} \mathcal{E}_{m}^{\|(1)}-\partial_{\|} v_{\|}^{(1)}-\mathcal{H}\left(b_{m}+1\right) v_{\|}^{(1)}+\Psi^{(1) \prime}\right]\right\}-2 v_{\perp o}^{i(1)} \\
& \times\left[\frac{1}{\mathcal{H}} \partial_{\perp i}\left(\Phi^{(1)}-v_{\|}^{(1)}\right)-\frac{\bar{\chi}}{\mathcal{H}} \frac{\mathrm{d}}{\mathrm{d} \bar{\chi}} \partial_{\perp i}\left(\Phi^{(1)}+v_{\|}^{(1)}\right)-4 \delta_{i l} S_{\perp}^{l(1)}\right]+\Phi^{(2)}+\Psi^{(2)}-\frac{1}{2} \hat{h}_{\|}^{(2)}+\frac{1}{\mathcal{H}} \Psi^{(2) \prime}-\frac{1}{2 \mathcal{H}} \hat{h}_{\|}^{(2) \prime} \\
& +2 \frac{\bar{a}^{2}}{\mathcal{H} \bar{\rho}_{m}}\left[\frac{1}{2} \mathcal{E}_{m}^{\|(2)}-\mathcal{E}_{m}^{0(1)} v_{\|}^{(1)}-\mathcal{E}_{m}^{\|(1)}\left(\delta_{m}^{(1)}-\Phi^{(1)}\right)\right]-2 b_{m}\left(\frac{1}{2} v_{\|}^{(2)}-\delta_{m}^{(1)} v_{\|}^{(1)}+2 \Phi^{(1)} v_{\|}^{(1)}\right)-\frac{2}{\mathcal{H}}\left[\frac{1}{2} \partial_{\|} v_{\|}^{(2)}-v_{\|}^{(1)} \partial_{\|} v_{\|}^{(1)}\right. \\
& \left.+\frac{\mathcal{H}}{2} v_{\|}^{(2)}-\frac{2}{\bar{\chi}}\left(v_{\|}^{(1)}\right)^{2}-v_{\|}^{(1)} \partial_{\perp j} v_{\perp}^{j(1)}-2 v_{\|}^{(1)} \Psi^{(1) \prime}-\Phi^{(1)} \partial_{\|} \Phi^{(1)}+2 \Psi^{(1)} \partial_{\|} \Phi^{(1)}\right]-4\left(\Phi^{(1)}\right)^{2}+4\left(\Psi^{(1)}\right)^{2} \\
& -\frac{2}{\mathcal{H}} \frac{\mathrm{d}}{\mathrm{d} \bar{\chi}}\left[\frac{7}{2}\left(\Phi^{(1)}\right)^{2}-\Phi^{(1)} v_{\|}^{(1)}+\frac{1}{2}\left(v_{\|}^{(1)}\right)^{2}-v_{\|}^{(1)} \Psi^{(1)}+\frac{1}{2} v_{\perp i}^{(1)} v_{\perp}^{i(1)}\right]+\frac{4}{\mathcal{H}} \Phi^{(1)}\left[2 \partial_{\|} \Phi^{(1)}-\left(\Phi^{(1) \prime}-\Psi^{(1) \prime}\right)\right] \\
& +\frac{2}{\mathcal{H}^{2}}\left(\Phi^{(1)}-v_{\|}^{(1)}\right) \frac{\mathrm{d}}{\mathrm{d} \bar{\chi}}\left[\frac{\bar{a}^{2}}{\bar{\rho}_{m}} \mathcal{E}_{m}^{\|(1)}-\partial_{\|} v_{\|}^{(1)}-\mathcal{H}\left(b_{m}+1\right) v_{\|}^{(1)}+\Psi^{(1) \prime}\right]-\frac{2}{\mathcal{H}}\left(3 \Phi^{(1)}-2 \Psi^{(1)}-v_{\|}^{(1)}\right)\left(\Phi^{(1) \prime}+\Psi^{(1) \prime}\right) \\
& +2\left\{\frac{1}{\mathcal{H}}\left(\Phi^{(1)}+\Psi^{(1)}\right)+\frac{\mathcal{H}^{\prime}}{\mathcal{H}^{3}}\left(\Phi^{(1)}-v_{\|}^{(1)}\right)+\frac{1}{\mathcal{H}^{2}}\left[\frac{\bar{a}^{2}}{\bar{\rho}_{m}} \mathcal{E}_{m}^{\|(1)}-\partial_{\|} v_{\|}^{(1)}-\mathcal{H}\left(b_{m}+1\right) v_{\|}^{(1)}+\Psi^{(1) \prime}\right]\right\}\left[\frac{\bar{a}^{2}}{\bar{\rho}_{m}} \mathcal{E}_{m}^{\|(1)}-\partial_{\|} v_{\|}^{(1)}\right. \\
& \left.-\mathcal{H}\left(b_{m}+1\right) v_{\|}^{(1)}+\Psi^{(1) \prime}\right]+\frac{2}{\mathcal{H}}\left[2 \frac{\mathrm{d}}{\mathrm{d} \bar{\chi}} \Phi^{(1)}-\partial_{\|}\left(\Phi^{(1)}+v_{\|}^{(1)}\right)\right]\left(\Phi^{(1)}+\Psi^{(1)}\right)+\frac{2}{\mathcal{H}} v_{\perp i}^{(1)} \partial_{\perp}^{i}\left(\Phi^{(1)}+\Psi^{(1)}\right) \\
& +\frac{2}{\mathcal{H}} \frac{\mathrm{d}}{\mathrm{d} \bar{\chi}} \partial_{\|}\left(\Phi^{(1)}+v_{\|}^{(1)}\right) T^{(1)}+4\left\{\left(-\frac{\mathcal{H}^{\prime}}{\mathcal{H}^{3}}+\frac{1}{\mathcal{H}}\right)\left[\frac{\bar{a}^{2}}{\bar{\rho}_{m}} \mathcal{E}_{m}^{\|(1)}-\partial_{\|} v_{\|}^{(1)}-\mathcal{H}\left(b_{m}+1\right) v_{\|}^{(1)}+\Psi^{(1) \prime}\right]\right. \\
& \left.-\frac{1}{\mathcal{H}^{2}} \frac{\mathrm{d}}{\mathrm{d} \bar{\chi}}\left[\frac{\bar{a}^{2}}{\bar{\rho}_{m}} \mathcal{E}_{m}^{\|(1)}-\partial_{\|} v_{\|}^{(1)}-\mathcal{H}\left(b_{m}+1\right) v_{\|}^{(1)}+\Psi^{(1) \prime}\right]+\Phi^{(1)}+\Psi^{(1)}\right\} I^{(1)}+2\left\{\frac{\mathrm{d}}{\mathrm{d} \bar{\chi}}\left(\Phi^{(1)}+\Psi^{(1)}\right)\right. \\
& \left.+\frac{1}{\mathcal{H}} \frac{\mathrm{d}}{\mathrm{d} \bar{\chi}}\left[2 \partial_{\|} \Phi^{(1)}-\left(\Phi^{(1) \prime}-\Psi^{(1) \prime}\right)\right]\right\} \int_{0}^{\bar{\chi}} \mathrm{d} \tilde{\chi}\left[2 \Phi^{(1)}+(\bar{\chi}-\tilde{\chi})\left(\Phi^{(1) \prime}+\Psi^{(1) \prime}\right)\right]+4\left[\frac{1}{\mathcal{H}} \partial_{\perp i}\left(\Phi^{(1)}-v_{\|}^{(1)}\right)\right. \\
& \left.+\frac{1}{\mathcal{H} \bar{\chi}} v_{\perp i}^{(1)}-\frac{1}{\mathcal{H}} \frac{\mathrm{d}}{\mathrm{d} \bar{\chi}} v_{\perp i}^{(1)}-2 S_{\perp}^{j(1)} \delta_{i j}\right] S_{\perp}^{i(1)}+\frac{2}{\mathcal{H}} \frac{\mathrm{d}}{\mathrm{d} \bar{\chi}}\left[\partial_{\perp i}\left(\Phi^{(1)}+v_{\|}^{(1)}\right)-\frac{1}{\bar{\chi}} v_{\perp i}^{(1)}\right] \\
& \times \int_{0}^{\bar{\chi}} \mathrm{d} \tilde{\chi}\left[(\bar{\chi}-\tilde{\chi}) \tilde{\partial}_{\perp}^{i}\left(\Phi^{(1)}+\Psi^{(1)}\right)\right]-\frac{\mathcal{H}^{\prime}}{\mathcal{H}^{2}} \Delta \ln a^{(2)}+\left[-\frac{\mathcal{H}^{\prime \prime}}{\mathcal{H}^{3}}+3\left(\frac{\mathcal{H}^{\prime}}{\mathcal{H}^{2}}\right)^{2}+\frac{\mathcal{H}^{\prime}}{\mathcal{H}^{2}}\right]\left(\Delta \ln a^{(1)}\right)^{2} \\
& +2\left\{-\frac{\mathcal{H}^{\prime}}{\mathcal{H}^{2}}\left(\Phi^{(1)}+\Psi^{(1)}\right)-\frac{1}{\mathcal{H}} \frac{\mathrm{d}}{\mathrm{d} \bar{\chi}}\left(\Phi^{(1)}+\Psi^{(1)}\right)-\left(\frac{\mathcal{H}^{\prime}}{\mathcal{H}^{3}}+\frac{1}{\mathcal{H}}\right)\left[\frac{\bar{a}^{2}}{\bar{\rho}_{m}} \mathcal{E}_{m}^{\|(1)}-\partial_{\|} v_{\|}^{(1)}-\mathcal{H}\left(b_{m}+1\right) v_{\|}^{(1)}\right.\right. \\
& \left.\left.+\Psi^{(1) \prime}\right]\right\} \Delta \ln a^{(1)}+4 \int_{0}^{\bar{\chi}} \mathrm{d} \tilde{\chi}\left\{\left(\Phi^{(1)}+\Psi^{(1)}\right)\left[\left(\Phi^{(1) \prime}+\Psi^{(1) \prime}\right)+\frac{\mathrm{d}}{\mathrm{d} \tilde{\chi}} \Phi^{(1)}\right]\right\}+\partial_{\|} \Delta x_{\| \text {post-Born }}^{(2)} .
\end{aligned}
$$


The last equation can be further expanded, applying again Eq. (B8):

$$
\begin{aligned}
& \frac{1}{\mathcal{H}} \frac{\mathrm{d}}{\mathrm{d} \bar{\chi}} v_{\perp i}^{(1)}=\frac{1}{\mathcal{H}} \frac{\bar{a}^{2}}{\bar{\rho}_{m}} \mathcal{P}_{i j} \mathcal{E}_{m}^{j(1)}-\frac{1}{\mathcal{H}} \partial_{\|} v_{\perp i}^{(1)}-\left(b_{m}+1\right) v_{\perp i}^{(1)}-\frac{1}{\mathcal{H}} \partial_{\perp i} \Phi^{(1)} \\
& \frac{2}{\mathcal{H}} \frac{\mathrm{d}}{\mathrm{d} \bar{\chi}} \partial_{\|}\left(\Phi^{(1)}+v_{\|}^{(1)}\right) T^{(1)}=+\frac{2}{\mathcal{H}}\left[-\frac{\bar{a}^{2}}{\bar{\rho}_{m}} \partial_{\|} \mathcal{E}_{m}^{\|(1)}+\partial_{\|}^{2} v_{\|}^{(1)}+\mathcal{H}\left(b_{m}+1\right) \partial_{\|} v_{\|}^{(1)}-\partial_{\|} \Phi^{(1) \prime}+2 \partial_{\|}^{2} \Phi^{(1)}\right] T^{(1)} \\
& \frac{\bar{\chi}}{\mathcal{H}} \frac{\mathrm{d}}{\mathrm{d} \bar{\chi}} \partial_{\perp i}\left(\Phi^{(1)}+v_{\|}^{(1)}\right)=-\frac{\bar{\chi}}{\mathcal{H}} \partial_{\perp i}\left(-\Phi^{(1) \prime}-\frac{\bar{a}^{2}}{\bar{\rho}_{m}} \mathcal{E}_{m}^{\|(1)}+\partial_{\|} v_{\|}^{(1)}+\mathcal{H}\left(b_{m}+1\right) v_{\|}^{(1)}+2 \partial_{\|} \Phi^{(1)}\right) \\
& +\frac{1}{\mathcal{H}} \partial_{\perp i}\left(\Phi^{(1)}+v_{\|}^{(1)}\right) \\
& \frac{2}{\mathcal{H}} \frac{\mathrm{d}}{\mathrm{d} \bar{\chi}}\left[\partial_{\perp i}\left(\Phi^{(1)}+v_{\|}^{(1)}\right)-\frac{1}{\bar{\chi}} v_{\perp i}^{(1)}\right]=\frac{2}{\mathcal{H}}\left[\partial_{\perp i}\left(-\Phi^{(1) \prime}-\frac{\bar{a}^{2}}{\bar{\rho}_{m}} \mathcal{E}_{m}^{\|(1)}+\partial_{\|} v_{\|}^{(1)}+\mathcal{H}\left(b_{m}+1\right) v_{\|}^{(1)}+2 \partial_{\|} \Phi^{(1)}\right)\right. \\
& \left.+\frac{1}{\bar{\chi}}\left(-2 \partial_{\perp i} \Phi^{(1)}-\partial_{\|} v_{\perp i}^{(1)}-\partial_{\perp i} v_{\|}^{(1)}+\frac{\bar{a}^{2}}{\bar{\rho}_{m}} \mathcal{P}_{i j} \mathcal{E}_{m}^{j(1)}-\mathcal{H}\left(b_{m}+1\right) v_{\perp i}^{(1)}\right)+\frac{1}{\bar{\chi}^{2}} v_{\perp i}^{(1)}\right] \\
& \frac{\mathrm{d}}{\mathrm{d} \bar{\chi}}\left[\frac{\bar{a}^{2}}{\bar{\rho}_{m}} \mathcal{E}_{m}^{\|(1)}-\partial_{\|} v_{\|}^{(1)}-\mathcal{H}\left(b_{m}+1\right) v_{\|}^{(1)}+\Psi^{(1) \prime}\right]=-\left(\frac{\bar{a}^{2}}{\bar{\rho}_{m}} \mathcal{E}_{m}^{\|(1)}\right)^{\prime}+\frac{\bar{a}^{2} \mathcal{H}}{\bar{\rho}_{m}}\left(b_{m}+1\right) \mathcal{E}_{m}^{\|(1)}+\mathcal{H}^{2} \frac{\mathrm{d} b_{m}}{\mathrm{~d} \ln a} v_{\|}^{(1)} \\
& +b_{m}\left[-\mathcal{H}^{2}\left(b_{m}+1\right) v_{\|}^{(1)}-2 \mathcal{H} \partial_{\|} v_{\|}^{(1)}+\left(\mathcal{H}^{\prime}-\mathcal{H}^{2}\right) v_{\|}^{(1)}-\mathcal{H} \partial_{\|} \Phi^{(1)}\right]-2 \mathcal{H} \partial_{\|} v_{\|}^{(1)}-\partial_{\|}^{2} \Phi^{(1)}-\partial_{\|}^{2} v_{\|}^{(1)} \\
& +\left(\mathcal{H}^{\prime}-\mathcal{H}^{2}\right) v_{\|}^{(1)}-\mathcal{H} \partial_{\|} \Phi^{(1)}+\frac{\mathrm{d}}{\mathrm{d} \bar{\chi}} \Psi^{(1) \prime}, \\
& \frac{\mathrm{d}}{\mathrm{d} \bar{\chi}}\left[\frac{7}{2}\left(\Phi^{(1)}\right)^{2}-\Phi^{(1)} v_{\|}^{(1)}+\frac{1}{2}\left(v_{\|}^{(1)}\right)^{2}-v_{\|}^{(1)} \Psi^{(1)}+\frac{1}{2} v_{\perp i}^{(1)} v_{\perp}^{i(1)}\right]=6 \Phi^{(1)} \frac{\mathrm{d}}{\mathrm{d} \bar{\chi}} \Phi^{(1)}-\Phi^{(1)} \Phi^{(1) \prime}+v_{\|}^{(1)} \Phi^{(1) \prime} \\
& -v_{\|}^{(1)} \frac{\mathrm{d}}{\mathrm{d} \bar{\chi}} \Psi^{(1)}-\Phi^{(1)} \partial_{\|} v_{\|}^{(1)}-\mathcal{H} \Phi^{(1)} v_{\|}^{(1)}+v_{\|}^{(1)} \partial_{\|} v_{\|}^{(1)}+\mathcal{H}\left(v_{\|}^{(1)}\right)^{2}-\Psi^{(1)} \partial_{\|} \Phi^{(1)}-\Psi^{(1)} \partial_{\|} v_{\|}^{(1)}-\mathcal{H} v_{\|}^{(1)} \Psi^{(1)} \\
& +v_{\perp i}^{(1)} \partial_{\|} v_{\perp}^{i(1)}+\mathcal{H} v_{\perp i}^{(1)} v_{\perp}^{i(1)}+v_{\perp i}^{(1)} \partial_{\perp}^{i} \Phi^{(1)}-\left(\Phi^{(1)}-v_{\|}^{(1)}+\Psi^{(1)}\right)\left(\frac{\bar{a}^{2}}{\bar{\rho}_{m}} \mathcal{E}_{m}^{\|(1)}-\mathcal{H} b_{m} v_{\|}^{(1)}\right) \\
& -v_{\perp i}^{(1)}\left(\frac{\bar{a}^{2}}{\bar{\rho}_{m}} \mathcal{P}_{j}^{i} \mathcal{E}_{m}^{j(1)}-\mathcal{H} b_{m} v_{\perp}^{i(1)}\right)
\end{aligned}
$$




$$
\begin{aligned}
& \partial_{\|} \Delta x_{\|}^{(2)}=\left(\Phi_{o}^{(1)}\right)^{2}+2 \Phi_{o}^{(1)} v_{\| o}^{(1)}+2 v_{\| o}^{(1)} \Psi_{o}^{(1)}-\left(\Psi_{o}^{(1)}\right)^{2}-v_{\perp k o}^{(1)} v_{\perp o}^{k(1)}+2\left(\Phi_{o}^{(1)}-v_{\| o}^{(1)}\right)\left\{( - \frac { \mathcal { H } ^ { \prime } } { \mathcal { H } ^ { 3 } } + \frac { 1 } { \mathcal { H } } ) \left[\frac{\bar{a}^{2}}{\bar{\rho}_{m}} \mathcal{E}_{m}^{\|(1)}\right.\right. \\
& \left.-\partial_{\|} v_{\|}^{(1)}-\mathcal{H}\left(b_{m}+1\right) v_{\|}^{(1)}+\Psi^{(1) \prime}\right]-\frac{\bar{\chi}}{\mathcal{H}} \frac{\mathrm{d}}{\mathrm{d} \bar{\chi}}\left[2 \partial_{\|} \Phi^{(1)}-\left(\Phi^{(1) \prime}-\Psi^{(1) \prime}\right)\right]+\Phi^{(1)}+\Psi^{(1)}-\bar{\chi} \frac{\mathrm{d}}{\mathrm{d} \bar{\chi}}\left(\Phi^{(1)}+\Psi^{(1)}\right) \\
& -\frac{1}{\mathcal{H}^{2}}\left[-2 \mathcal{H} \partial_{\|} v_{\|}^{(1)}-\partial_{\|}^{2} \Phi^{(1)}-\partial_{\|}^{2} v_{\|}^{(1)}+\left(\mathcal{H}^{\prime}-\mathcal{H}^{2}\right) v_{\|}^{(1)}-\mathcal{H} \partial_{\|} \Phi^{(1)}+\frac{\mathrm{d}}{\mathrm{d} \bar{\chi}} \Psi^{(1) \prime}\right]-\frac{1}{\mathcal{H}^{2}}\left[-\left(\frac{\bar{a}^{2}}{\bar{\rho}_{m}} \mathcal{E}_{m}^{\|(1)}\right)^{\prime}\right. \\
& \left.\left.+\frac{\bar{a}^{2} \mathcal{H}}{\bar{\rho}_{m}}\left(b_{m}+1\right) \mathcal{E}_{m}^{\|(1)}+\mathcal{H}^{2} \frac{\mathrm{d} b_{m}}{\mathrm{~d} \ln a} v_{\|}^{(1)}-\mathcal{H}^{2} b_{m}\left(b_{m}+1\right) v_{\|}^{(1)}-2 \mathcal{H} b_{m} \partial_{\|} v_{\|}^{(1)}+\left(\mathcal{H}^{\prime}-\mathcal{H}^{2}\right) b_{m} v_{\|}^{(1)}-\mathcal{H} b_{m} \partial_{\|} \Phi^{(1)}\right]\right\} \\
& -2 v_{\perp o}^{i(1)}\left[+\frac{2}{\mathcal{H}} \partial_{\perp i} \Phi^{(1)}-\frac{\bar{\chi}}{\mathcal{H}} \partial_{\perp i}\left(-\Phi^{(1) \prime}-\frac{\bar{a}^{2}}{\bar{\rho}_{m}} \mathcal{E}_{m}^{\|(1)}+\partial_{\|} v_{\|}^{(1)}+\mathcal{H}\left(b_{m}+1\right) v_{\|}^{(1)}+2 \partial_{\|} \Phi^{(1)}\right)-4 \delta_{i l} S_{\perp}^{l(1)}\right] \\
& +\Phi^{(2)}+\Psi^{(2)}-\frac{1}{2} \hat{h}_{\|}^{(2)}+\frac{1}{\mathcal{H}} \Psi^{(2) \prime}-\frac{1}{2 \mathcal{H}} \hat{h}_{\|}^{(2) \prime}-\frac{2}{\mathcal{H}}\left[\frac{1}{2} \partial_{\|} v_{\|}^{(2)}-v_{\|}^{(1)} \partial_{\|} v_{\|}^{(1)}+\frac{\mathcal{H}}{2} v_{\|}^{(2)}-\frac{2}{\bar{\chi}}\left(v_{\|}^{(1)}\right)^{2}-v_{\|}^{(1)} \partial_{\perp j} v_{\perp}^{j(1)}\right. \\
& \left.-2 v_{\|}^{(1)} \Psi^{(1) \prime}-\Phi^{(1)} \partial_{\|} \Phi^{(1)}+2 \Psi^{(1)} \partial_{\|} \Phi^{(1)}\right]-4\left(\Phi^{(1)}\right)^{2}+4\left(\Psi^{(1)}\right)^{2}-\frac{2}{\mathcal{H}}\left[6 \Phi^{(1)} \frac{\mathrm{d}}{\mathrm{d} \bar{\chi}} \Phi^{(1)}-\Phi^{(1)} \Phi^{(1) \prime}+v_{\|}^{(1)} \Phi^{(1) \prime}\right. \\
& -v_{\|}^{(1)} \frac{\mathrm{d}}{\mathrm{d} \bar{\chi}} \Psi^{(1)}-\Phi^{(1)} \partial_{\|} v_{\|}^{(1)}-\mathcal{H} \Phi^{(1)} v_{\|}^{(1)}+v_{\|}^{(1)} \partial_{\|} v_{\|}^{(1)}+\mathcal{H}\left(v_{\|}^{(1)}\right)^{2}-\Psi^{(1)} \partial_{\|} \Phi^{(1)}-\Psi^{(1)} \partial_{\|} v_{\|}^{(1)}-\mathcal{H} v_{\|}^{(1)} \Psi^{(1)}+v_{\perp i}^{(1)} \partial_{\|} v_{\perp}^{i(1)} \\
& \left.+\mathcal{H} v_{\perp i}^{(1)} v_{\perp}^{i(1)}+v_{\perp i}^{(1)} \partial_{\perp}^{i} \Phi^{(1)}-\left(\Phi^{(1)}-v_{\|}^{(1)}+\Psi^{(1)}\right)\left(\frac{\bar{a}^{2}}{\bar{\rho}_{m}} \mathcal{E}_{m}^{\|(1)}-\mathcal{H} b_{m} v_{\|}^{(1)}\right)-v_{\perp i}^{(1)}\left(\frac{\bar{a}^{2}}{\bar{\rho}_{m}} \mathcal{P}_{j}^{i} \mathcal{E}_{m}^{j(1)}-\mathcal{H} b_{m} v_{\perp}^{i(1)}\right)\right] \\
& +2 \frac{\bar{a}^{2}}{\mathcal{H} \bar{\rho}_{m}}\left[\frac{1}{2} \mathcal{E}_{m}^{\|(2)}-\mathcal{E}_{m}^{0(1)} v_{\|}^{(1)}-\mathcal{E}_{m}^{\|(1)}\left(\delta_{m}^{(1)}-\Phi^{(1)}\right)\right]-2 b_{m}\left(\frac{1}{2} v_{\|}^{(2)}-\delta_{m}^{(1)} v_{\|}^{(1)}+2 \Phi^{(1)} v_{\|}^{(1)}\right) \\
& +\frac{2}{\mathcal{H}^{2}}\left(\Phi^{(1)}-v_{\|}^{(1)}\right)\left[-\left(\frac{\bar{a}^{2}}{\bar{\rho}_{m}} \mathcal{E}_{m}^{\|(1)}\right)^{\prime}+\frac{\bar{a}^{2} \mathcal{H}}{\bar{\rho}_{m}}\left(b_{m}+1\right) \mathcal{E}_{m}^{\|(1)}+\mathcal{H}^{2} \frac{\mathrm{d} b_{m}}{\mathrm{~d} \ln a} v_{\|}^{(1)}-\mathcal{H}^{2} b_{m}\left(b_{m}+1\right) v_{\|}^{(1)}-2 \mathcal{H} b_{m} \partial_{\|} v_{\|}^{(1)}\right. \\
& \left.+\left(\mathcal{H}^{\prime}-\mathcal{H}^{2}\right) b_{m} v_{\|}^{(1)}-\mathcal{H} b_{m} \partial_{\|} \Phi^{(1)}-2 \mathcal{H} \partial_{\|} v_{\|}^{(1)}-\partial_{\|}^{2} \Phi^{(1)}-\partial_{\|}^{2} v_{\|}^{(1)}+\left(\mathcal{H}^{\prime}-\mathcal{H}^{2}\right) v_{\|}^{(1)}-\mathcal{H} \partial_{\|} \Phi^{(1)}+\frac{\mathrm{d}}{\mathrm{d} \bar{\chi}} \Psi^{(1) \prime}\right] \\
& +\frac{4}{\mathcal{H}} \Phi^{(1)}\left[2 \partial_{\|} \Phi^{(1)}-\left(\Phi^{(1) \prime}-\Psi^{(1) \prime}\right)\right]-\frac{2}{\mathcal{H}}\left(3 \Phi^{(1)}-2 \Psi^{(1)}-v_{\|}^{(1)}\right)\left(\Phi^{(1) \prime}+\Psi^{(1) \prime}\right)+2\left\{\frac{1}{\mathcal{H}}\left(\Phi^{(1)}+\Psi^{(1)}\right)\right. \\
& \left.+\frac{\mathcal{H}^{\prime}}{\mathcal{H}^{3}}\left(\Phi^{(1)}-v_{\|}^{(1)}\right)+\frac{1}{\mathcal{H}^{2}}\left[\frac{\bar{a}^{2}}{\bar{\rho}_{m}} \mathcal{E}_{m}^{\|(1)}-\partial_{\|} v_{\|}^{(1)}-\mathcal{H}\left(b_{m}+1\right) v_{\|}^{(1)}+\Psi^{(1) \prime}\right]\right\}\left[\frac{\bar{a}^{2}}{\bar{\rho}_{m}} \mathcal{E}_{m}^{\|(1)}-\partial_{\|} v_{\|}^{(1)}-\mathcal{H}\left(b_{m}+1\right) v_{\|}^{(1)}\right. \\
& \left.+\Psi^{(1) \prime}\right]+\frac{2}{\mathcal{H}}\left[2 \frac{\mathrm{d}}{\mathrm{d} \bar{\chi}} \Phi^{(1)}-\partial_{\|}\left(\Phi^{(1)}+v_{\|}^{(1)}\right)\right]\left(\Phi^{(1)}+\Psi^{(1)}\right)+\frac{2}{\mathcal{H}} v_{\perp i}^{(1)} \partial_{\perp}^{i}\left(\Phi^{(1)}+\Psi^{(1)}\right)+\frac{2}{\mathcal{H}} \frac{\mathrm{d}}{\mathrm{d} \bar{\chi}} \partial_{\|}\left(\Phi^{(1)}+v_{\|}^{(1)}\right) T^{(1)} \\
& +4\left\{\left(-\frac{\mathcal{H}^{\prime}}{\mathcal{H}^{3}}+\frac{1}{\mathcal{H}}\right)\left[\frac{\bar{a}^{2}}{\bar{\rho}_{m}} \mathcal{E}_{m}^{\|(1)}-\partial_{\|} v_{\|}^{(1)}-\mathcal{H}\left(b_{m}+1\right) v_{\|}^{(1)}+\Psi^{(1) \prime}\right]-\frac{1}{\mathcal{H}^{2}}\left[-\left(\frac{\bar{a}^{2}}{\bar{\rho}_{m}} \mathcal{E}_{m}^{\|(1)}\right)^{\prime}+\frac{\bar{a}^{2} \mathcal{H}}{\bar{\rho}_{m}}\left(b_{m}+1\right) \mathcal{E}_{m}^{\|(1)}\right.\right. \\
& +\mathcal{H}^{2} \frac{\mathrm{d} b_{m}}{\mathrm{~d} \ln a} v_{\|}^{(1)}+b_{m}\left[-\mathcal{H}^{2}\left(b_{m}+1\right) v_{\|}^{(1)}-2 \mathcal{H} \partial_{\|} v_{\|}^{(1)}+\left(\mathcal{H}^{\prime}-\mathcal{H}^{2}\right) v_{\|}^{(1)}-\mathcal{H} \partial_{\|} \Phi^{(1)}\right]-2 \mathcal{H} \partial_{\|} v_{\|}^{(1)}-\partial_{\|}^{2} \Phi^{(1)}-\partial_{\|}^{2} v_{\|}^{(1)} \\
& \left.\left.+\left(\mathcal{H}^{\prime}-\mathcal{H}^{2}\right) v_{\|}^{(1)}-\mathcal{H} \partial_{\|} \Phi^{(1)}+\frac{\mathrm{d}}{\mathrm{d} \bar{\chi}} \Psi^{(1) \prime}\right]+\Phi^{(1)}+\Psi^{(1)}\right\} I^{(1)}+2\left[\frac{\mathrm{d}}{\mathrm{d} \bar{\chi}}\left(\Phi^{(1)}+\Psi^{(1)}\right)+\frac{1}{\mathcal{H}} \frac{\mathrm{d}}{\mathrm{d} \bar{\chi}}\left(2 \partial_{\|} \Phi^{(1)}-\Phi^{(1) \prime}\right.\right. \\
& \left.\left.+\Psi^{(1) \prime}\right)\right] \int_{0}^{\bar{\chi}} \mathrm{d} \tilde{\chi}\left[2 \Phi^{(1)}+(\bar{\chi}-\tilde{\chi})\left(\Phi^{(1) \prime}+\Psi^{(1) \prime}\right)\right]+4\left[\frac{1}{\mathcal{H}} \partial_{\perp i}\left(\Phi^{(1)}-v_{\|}^{(1)}\right)+\frac{1}{\mathcal{H} \bar{\chi}} v_{\perp i}^{(1)}+\frac{1}{\mathcal{H}} \frac{\bar{a}^{2}}{\bar{\rho}_{m}} \mathcal{P}_{i j} \mathcal{E}_{m}^{j(1)}-\frac{1}{\mathcal{H}} \partial_{\|} v_{\perp i}^{(1)}\right. \\
& \left.-\left(b_{m}+1\right) v_{\perp i}^{(1)}-\frac{1}{\mathcal{H}} \partial_{\perp i} \Phi^{(1)}-2 S_{\perp}^{j(1)} \delta_{i j}\right] S_{\perp}^{i(1)}+\frac{2}{\mathcal{H}}\left[\partial_{\perp i}\left(-\Phi^{(1) \prime}-\frac{\bar{a}^{2}}{\bar{\rho}_{m}} \mathcal{E}_{m}^{\|(1)}+\partial_{\|} v_{\|}^{(1)}+\mathcal{H}\left(b_{m}+1\right) v_{\|}^{(1)}+2 \partial_{\|} \Phi^{(1)}\right)\right. \\
& \left.+\frac{1}{\bar{\chi}}\left(-2 \partial_{\perp i} \Phi^{(1)}-\partial_{\perp i} v_{\|}^{(1)}-\partial_{\|} v_{\perp i}^{(1)}+\frac{\bar{a}^{2}}{\bar{\rho}_{m}} \mathcal{P}_{i j} \mathcal{E}_{m}^{j(1)}-\mathcal{H}\left(b_{m}+1\right) v_{\perp i}^{(1)}\right)+\frac{1}{\bar{\chi}^{2}} v_{\perp i}^{(1)}\right] \int_{0}^{\bar{\chi}} \mathrm{d} \tilde{\chi}\left[(\bar{\chi}-\tilde{\chi}) \tilde{\partial}_{\perp}^{i}\left(\Phi^{(1)}+\Psi^{(1)}\right)\right] \\
& -\frac{\mathcal{H}^{\prime}}{\mathcal{H}^{2}} \Delta \ln a^{(2)}+\left[-\frac{\mathcal{H}^{\prime \prime}}{\mathcal{H}^{3}}+3\left(\frac{\mathcal{H}^{\prime}}{\mathcal{H}^{2}}\right)^{2}+\frac{\mathcal{H}^{\prime}}{\mathcal{H}^{2}}\right]\left(\Delta \ln a^{(1)}\right)^{2}+2\left\{-\frac{\mathcal{H}^{\prime}}{\mathcal{H}^{2}}\left(\Phi^{(1)}+\Psi^{(1)}\right)-\frac{1}{\mathcal{H}} \frac{\mathrm{d}}{\mathrm{d} \bar{\chi}}\left(\Phi^{(1)}+\Psi^{(1)}\right)\right. \\
& \left.-\left(\frac{\mathcal{H}^{\prime}}{\mathcal{H}^{3}}+\frac{1}{\mathcal{H}}\right)\left[\frac{\bar{a}^{2}}{\bar{\rho}_{m}} \mathcal{E}_{m}^{\|(1)}-\partial_{\|} v_{\|}^{(1)}-\mathcal{H}\left(b_{m}+1\right) v_{\|}^{(1)}+\Psi^{(1) \prime}\right]\right\} \Delta \ln a^{(1)}+4 \int_{0}^{\bar{\chi}} \mathrm{d} \tilde{\chi}\left\{( \Phi ^ { ( 1 ) } + \Psi ^ { ( 1 ) } ) \left[\left(\Phi^{(1) \prime}+\Psi^{(1) \prime}\right)\right.\right. \\
& \left.\left.+\frac{\mathrm{d}}{\mathrm{d} \tilde{\chi}} \Phi^{(1)}\right]\right\} \text {. }
\end{aligned}
$$


Finally we obtain

$$
\begin{aligned}
& \Delta_{g}^{(2)}=\delta_{g}^{(2)}+v_{\|}^{(2)}-3 \Psi^{(2)}+b_{e} \Delta \ln a^{(2)}+\partial_{\|} \Delta x_{\|}^{(2)}+\frac{2}{\bar{\chi}} \Delta x_{\|}^{(2)}-2 \kappa^{(2)}+\left(\Delta_{g}^{(1)}\right)^{2} \\
& -\left(\delta_{g}^{(1)}\right)^{2}-3\left(\Phi^{(1)}\right)^{2}-\left(v_{\|}^{(1)}\right)^{2}+2 \Phi^{(1)} v_{\|}^{(1)}-9\left(\Psi^{(1)}\right)^{2}-\frac{1}{\mathcal{H}^{2}}\left(\partial_{\|} v_{\|}^{(1)}\right)^{2}-\frac{1}{\mathcal{H}^{2}}\left(\Psi^{(1) \prime}\right)^{2}-6 \Phi^{(1)} \Psi^{(1)} \\
& -\frac{2}{\mathcal{H}} \Phi^{(1)} \Psi^{(1) \prime}-\frac{2}{\mathcal{H}} v_{\|}^{(1)} \partial_{\|} v_{\|}^{(1)}+\frac{2}{\mathcal{H}} v_{\|}^{(1)} \Psi^{(1) \prime}+\frac{2}{\mathcal{H}} \Psi^{(1)} \partial_{\|} v_{\|}^{(1)}-\frac{2}{\mathcal{H}} \Psi^{(1)} \Psi^{(1) \prime}+\frac{2}{\mathcal{H}^{2}} \Psi^{(1) \prime} \partial_{\|} v_{\|}^{(1)}+\frac{2}{\mathcal{H}} \Phi^{(1)} \partial_{\|} v_{\|}^{(1)} \\
& +v_{\perp i}^{(1)} v_{\perp}^{i(1)}-8\left(I^{(1)}\right)^{2}+8 \Phi^{(1)} I^{(1)}+8 \Psi^{(1)} I^{(1)}+2 \partial_{\|}\left(+3 \Psi^{(1)}-v_{\|}^{(1)}-\delta_{g}^{(1)}\right) T^{(1)}-\frac{4}{\bar{\chi}} \kappa^{(1)} T^{(1)}-\frac{2}{\bar{\chi}^{2}}\left(T^{(1)}\right)^{2} \\
& +\frac{2}{\mathcal{H}}\left(-\partial_{\|} v_{\|}^{(1)}-\mathcal{H} v_{\|}^{(1)}-\partial_{\|} \Phi^{(1)}+3 \frac{\mathrm{d}}{\mathrm{d} \tilde{\chi}} \Psi^{(1)}-\frac{\mathrm{d}}{\mathrm{d} \bar{\chi}} \delta_{g}^{(1)}-\frac{2}{\bar{\chi}^{2}} T^{(1)}-\frac{2}{\bar{\chi}} \kappa^{(1)}\right) \Delta \ln a^{(1)} \\
& \left.+2 \frac{\mathcal{H}^{\prime}}{\mathcal{H}^{2}}\left(\Phi^{(1)}-v_{\|}^{(1)}+\Psi^{(1)}-\frac{1}{\mathcal{H}} \partial_{\|} v_{\|}^{(1)}+\frac{1}{\mathcal{H}} \Psi^{(1) \prime}\right) \Delta \ln a^{(1)}+\left(-b_{e}+\frac{\mathrm{d} \ln b_{e}}{\mathrm{~d} \ln \bar{a}}-\left(\frac{\mathcal{H}^{\prime}}{\mathcal{H}^{2}}\right)^{2}-\frac{2}{\bar{\chi}^{2} \mathcal{H}^{2}}\right)^{\left(\Delta \ln a^{(1)}\right.}\right)^{2} \\
& +2\left(\Phi^{(1)}+\Psi^{(1)}-2 I^{(1)}\right) \int_{0}^{\bar{\chi}} \mathrm{d} \tilde{\chi}\left[\frac{\tilde{\chi}}{\bar{\chi}}\left(2 \tilde{\partial}_{\|}+(\bar{\chi}-\tilde{\chi}) \mathcal{P}^{m n} \tilde{\partial}_{m} \tilde{\partial}_{n}\right)\left(\Phi^{(1)}+\Psi^{(1)}\right)\right] \\
& -\left[\int_{0}^{\bar{\chi}} \mathrm{d} \tilde{\chi} \frac{\tilde{\chi}}{\bar{\chi}}\left(\mathcal{P}_{j}^{i} \tilde{\partial}_{\|}+(\bar{\chi}-\tilde{\chi}) \mathcal{P}_{j}^{p} \mathcal{P}^{i q} \tilde{\partial}_{q} \tilde{\partial}_{p}\right)\left(\Phi^{(1)}+\Psi^{(1)}\right)\right] \\
& \times\left[\int_{0}^{\bar{\chi}} \mathrm{d} \tilde{\chi} \frac{\tilde{\chi}}{\bar{\chi}}\left(\mathcal{P}_{i}^{j} \tilde{\partial}_{\|}+(\bar{\chi}-\tilde{\chi}) \mathcal{P}_{i}^{n} \mathcal{P}^{j m} \tilde{\partial}_{m} \tilde{\partial}_{n}\right)\left(\Phi^{(1)}+\Psi^{(1)}\right)\right] \\
& -2 \partial_{\perp i}\left(v_{\|}^{(1)}-3 \Psi^{(1)}+\delta_{g}^{(1)}\right) \int_{0}^{\bar{\chi}} \mathrm{d} \tilde{\chi}\left[(\bar{\chi}-\tilde{\chi}) \tilde{\partial}_{\perp}^{i}\left(\Phi^{(1)}+\Psi^{(1)}\right)\right]+2 v_{\perp}^{i(1)} \partial_{\perp i} T^{(1)} \\
& +4 S_{\perp}^{i(1)}\left\{-\frac{1}{\bar{\chi}} \int_{0}^{\bar{\chi}} \mathrm{d} \tilde{\chi}\left[(\bar{\chi}-\tilde{\chi}) \tilde{\partial}_{\perp}^{i}\left(\Phi^{(1)}+\Psi^{(1)}\right)\right]+\partial_{\perp i}\left(\frac{1}{\mathcal{H}} \Delta \ln a^{(1)}+T^{(1)}\right)\right\} \\
& +2 v_{\perp i o}^{(1)} v_{\perp o}^{i(1)}-2\left(v_{\| o}^{(1)}\right)^{2}-2\left(\Phi_{o}^{(1)}\right)^{2}+4 v_{\| o}^{(1)} \Psi_{o}^{(1)}-2\left(\Psi_{o}^{(1)}\right)^{2}+4 \Phi_{o}^{(1)} v_{\| o}^{(1)}-4 \Phi_{o}^{(1)} \Psi_{o}^{(1)}-2 v_{\perp i o}^{(1)} \\
& \times\left\{\bar{\chi} \partial_{\perp}^{i}\left(v_{\|}^{(1)}-3 \Psi^{(1)}+\delta_{g}^{(1)}\right)+2 S_{\perp}^{i(1)}+\partial_{\perp}^{i}\left(\frac{1}{\mathcal{H}} \Delta \ln a^{(1)}+T^{(1)}\right)-\frac{1}{\bar{\chi}} \int_{0}^{\bar{\chi}} \mathrm{d} \tilde{\chi}\left[(\bar{\chi}-\tilde{\chi}) \tilde{\partial}_{\perp}^{i}\left(\Phi^{(1)}+\Psi^{(1)}\right)\right]\right\} \\
& +2\left(\Phi_{o}^{(1)}-v_{\| o}^{(1)}+\Psi_{o}^{(1)}\right)\left\{2\left(\Phi^{(1)}+\Psi^{(1)}-2 I^{(1)}\right)-\int_{0}^{\bar{\chi}} \mathrm{d} \tilde{\chi}\left[\frac{\tilde{\chi}}{\bar{\chi}}\left(2 \tilde{\partial}_{\|}+(\bar{\chi}-\tilde{\chi}) \mathcal{P}^{m n} \tilde{\partial}_{m} \tilde{\partial}_{n}\right)\left(\Phi^{(1)}+\Psi^{(1)}\right)\right]\right\} \\
& -2 \frac{\bar{a}^{2}}{\mathcal{H} \bar{\rho}_{m}}\left(\mathcal{E}_{m}^{\|(1)}-\frac{\mathcal{H}}{\bar{a}^{2}} \bar{\rho}_{m} b_{m} v_{\|}^{(1)}\right)\left[\Phi^{(1)}-v_{\|}^{(1)}+\Psi^{(1)}-\frac{1}{\mathcal{H}} \partial_{\|} v_{\|}^{(1)}+\frac{1}{\mathcal{H}} \Psi^{(1) \prime}-\left(1+\frac{\mathcal{H}^{\prime}}{\mathcal{H}^{2}}\right) \Delta \ln a^{(1)}\right] \\
& -\left(\frac{\bar{a}^{2}}{\mathcal{H} \bar{\rho}_{m}}\right)^{2}\left(\mathcal{E}_{m}^{\|(1)}-\frac{\mathcal{H}}{\bar{a}^{2}} \bar{\rho}_{m} b_{m} v_{\|}^{(1)}\right)^{2} \text {. }
\end{aligned}
$$

This is the main result in Poisson gauge with no velocity bias. If we explicitly identify the weak lensing shear and rotation contributions, we arrive at Eq. (B12) in Appendix B.

\section{PRESCRIPTION FOR THE GALAXY BIAS}

We need to relate the fluctuations of galaxy number density to the underlying matter density fluctuation $\delta_{m}$, assuming scale-independent bias. In order to define correctly the bias we have to choose an appropriate frame where the baryon velocity perturbations vanish. If we assume that both at first and second order the baryon rest frame coincides with the rest frame of CDM, the most general gauge that meets these requirements is the comoving-time orthogonal (CO) gauge (see e.g. 37), which becomes the usual comoving-synchronous gauge when the perturbations are dominated by pressure-free matter, for example in the $\Lambda \mathrm{CDM}$ model $^{8}$. In this frame, galaxy and matter over-

\footnotetext{
8 An analogous approach is used in 38.
} 
densities are gauge invariant [37. This gauge is defined by the conditions $B^{i(n)}=v^{i(n)}=0$. Then

$$
\mathrm{d} s^{2}=a(\eta)^{2}\left[-\left(1+2 \varphi^{(1)}+\varphi^{(2)}\right) \mathrm{d} \eta^{2}+\left(\delta_{i j}+h_{i j \mathrm{CO}}^{(1)}+\frac{1}{2} h_{i j \mathrm{CO}}^{(2)}\right) \mathrm{d} x^{i} \mathrm{~d} x^{j}\right]
$$

where $A_{\mathrm{CO}}^{(n)}=\varphi^{(n)}, h_{i j \mathrm{CO}}^{(n)}=-2 \psi^{(n)} \delta_{i j}+F_{i j \mathrm{CO}}^{(n)}$, with $F_{i j \mathrm{CO}}^{(n)}=\left(\partial_{i} \partial_{j}-\delta_{i j} \nabla^{2} / 3\right) \xi^{(n)}+\partial_{i} \hat{\xi}_{j}^{(n)}+\partial_{j} \hat{\xi}_{i}^{(n)}+\hat{h}_{i j}^{(n)}, \partial_{i} \hat{\xi}^{i(n)}=$ $\partial_{i} \hat{h}^{i j(n)}=0$. For simplicity, we neglect vector and tensor perturbations at first order, i.e. $\hat{\xi}_{j}^{(1)}=\hat{h}_{i j}^{(1)}=0$.

In order to find $\delta_{g} \mathrm{CO}$, we transform the metric perturbations from the Poisson gauge to the comoving-time orthogonal gauge. Using [36], we get, at first order,

$$
\delta_{g \mathrm{P}}^{(1)}=\delta_{g \mathrm{CO}}^{(1)}-b_{e} \mathcal{H} v_{\mathrm{P}}^{(1)}+3 \mathcal{H} v_{\mathrm{P}}^{(1)},
$$

and, at second order,

$$
\begin{aligned}
\delta_{g \mathrm{P}}^{(2)}= & \delta_{g \mathrm{CO}}^{(2)}-b_{e} \mathcal{H} v_{\mathrm{P}}^{(2)}+3 \mathcal{H} v_{\mathrm{P}}^{(2)}+\left(b_{e} \mathcal{H}^{\prime}-3 \mathcal{H}^{\prime}+\mathcal{H}^{2} \frac{\mathrm{d} b_{e}}{\mathrm{~d} \ln \bar{a}}+b_{e}^{2} \mathcal{H}^{2}-6 b_{e} \mathcal{H}^{2}+9 \mathcal{H}^{2}\right)\left(v_{\mathrm{P}}^{(1)}\right)^{2} \\
& +\mathcal{H} b_{e} v_{\mathrm{P}}^{(1)} v_{\mathrm{P}}^{(1)^{\prime}}-3 \mathcal{H} v_{\mathrm{P}}^{(1)} v_{\mathrm{P}}^{(1)^{\prime}}-2 \mathcal{H} b_{e} v_{\mathrm{P}}^{(1)} \delta_{g \mathrm{CO}}^{(1)}+6 \mathcal{H} v_{\mathrm{P}}^{(1)} \delta_{g \mathrm{CO}}^{(1)}-2 v_{\mathrm{P}}^{(1)} \delta_{g \mathrm{CO}}^{(1)}{ }^{\prime} \\
& -\frac{1}{2} \partial^{i} \xi^{(1)}\left(-b_{e} \mathcal{H} \partial_{i} v_{\mathrm{P}}^{(1)}+3 \mathcal{H} \partial_{i} v_{\mathrm{P}}^{(1)}+2 \partial_{i} \delta_{g \mathrm{CO}}^{(1)}\right)-\left(b_{e}-3\right) \mathcal{H} \nabla^{-2} \Xi
\end{aligned}
$$

Here

$$
\begin{aligned}
\Xi= & +v_{\mathrm{P}}^{(1)} \nabla^{2} v_{\mathrm{P}}^{(1)^{\prime}}-v_{\mathrm{P}}^{(1)^{\prime}} \nabla^{2} v_{\mathrm{P}}^{(1)}-2 \partial_{i} \Phi^{(1)} \partial^{i} v_{\mathrm{P}}^{(1)}-2 \Phi^{(1)} \nabla^{2} v_{\mathrm{P}}^{(1)}-4 \Psi^{(1)} \nabla^{2} v_{\mathrm{P}}^{(1)}-4 \partial_{i} \Psi^{(1)} \partial^{i} v_{\mathrm{P}}^{(1)} \\
& +\frac{1}{2} \partial_{i} \xi^{(1)} \partial^{i} \nabla^{2} v_{\mathrm{P}}^{(1)}+\frac{1}{2} \partial_{i} v_{\mathrm{P}}^{(1)} \partial^{i} \nabla^{2} \xi^{(1)}+\partial_{i} \partial_{j} \xi^{(1)} \partial^{i} \partial^{j} v_{\mathrm{P}}^{(1)} .
\end{aligned}
$$

(Another useful relation is $\xi^{(1)^{\prime}} / 2=v_{\mathrm{P}}^{(1)}$.)

The scale-independent bias at first and second order is defined by ${ }^{9}$

$$
\delta_{g \mathrm{CO}}^{(1)}+\frac{1}{2} \delta_{g \mathrm{CO}}^{(2)}=b_{1}^{L} \delta_{m \mathrm{CO}}^{(1)}+\frac{1}{2} b_{1}^{L} \delta_{m \mathrm{CO}}^{(2)}+\frac{1}{2} b_{2}^{L}\left(\delta_{m \mathrm{CO}}^{(1)}\right)^{2} .
$$

These are substituted into Eqs. (253) and (254), and then we can replace the term $\delta_{g}^{(2)}$ in the expression for the observed number overdensity at second order in Poisson gauge, Eq. (237). This allows us to relate the observed number counts to the underlying matter overdensity in a gauge invariant way.

\section{CONCLUSIONS}

We presented for the first time a derivation of the observed galaxy number counts to second order on cosmological scales, including all relativistic effects. Our results are given both in a general gauge and in Poisson gauge, and apply to general dark energy models, including those where dark energy interacts non-gravitationally with dark matter. Our results also apply to metric theories of modified gravity as an alternative to dark energy. The main, fully general, result for the galaxy number count fluctuations at second order is Eq. (158), which is specialized to the Poisson gauge in Eq. (237). We also gave these results in the form where the contribution from weak lensing shear and rotation is made explicit, in Eqs. (159) and (238).

We derived the expressions needed to relate the observed number over-density to the matter over-density via the bias in a gauge-invariant way, in Eqs. 253)-2256.

The second-order effects that we derive, especially those involving integrals along the line of sight, may make a non-negligible contribution to the observed number counts. This will be important for removing potential biases on parameter estimation in precision cosmology with galaxy surveys. It will also be important for an accurate analysis of the 'contamination' of primordial non-Gaussianity by relativistic projection effects. This is discussed in Paper I [20] and is the subject of ongoing work [39].

\footnotetext{
${ }^{9}$ A typo in this equation has been corrected.
} 


\section{Acknowledgments:}

We thank Enea di Dio, Ruth Durrer, Giovanni Marozzi, Obinna Umeh for helpful discussions. DB and RM are supported by the South African Square Kilometre Array Project. RM acknowledges support from the UK Science \& Technology Facilities Council (grant ST/K0090X/1). RM and CC are supported by the South African National Research Foundation. We thank Ruth Durrer for alerting us to the possibility of an error in our results.

\section{Appendix A: Perturbation terms in general gauge}

From Eq. 85 the perturbations of $g_{\mu \nu}$ and $g^{\mu \nu}$ are

$$
\begin{array}{ll}
g_{00}=a^{2} \hat{g}_{00}=-a^{2}\left(1+2 A^{(1)}+A^{(2)}\right), & g^{00}=a^{-2} \hat{g}^{00}=-a^{-2}\left[1-2 A^{(1)}-A^{(2)}+4\left(A^{(1)}\right)^{2}-B_{i}^{(1)} B^{i(1)}\right] \\
g_{0 i}=a^{2} \hat{g}_{0 i}=a^{2}\left(-B_{i}^{(1)}-B_{i}^{(2)} / 2\right), & g^{0 i}=a^{-2} \hat{g}^{0 i}=a^{-2}\left[-B^{i(1)}-B^{i(2)} / 2+2 A^{(1)} B^{i(1)}+B_{k}^{(1)} h^{k i(1)}\right], \\
g_{i j}=a^{2} \hat{g}_{i j}=a^{2}\left(\delta_{i j}+h_{i j}^{(1)}+h_{i j}^{(2)} / 2\right), & g^{i j}=a^{-2} \hat{g}^{i j}=a^{-2}\left[\delta^{i j}-h^{i j(1)}-h^{i j(2)} / 2+h^{i k(1)} h_{k}^{j(1)}-B^{i(1)} B^{j(1)}\right],
\end{array}
$$

For Christoffel symbols $\Gamma_{\rho \sigma}^{\mu}=\Gamma_{\rho \sigma}^{\mu(0)}+\Gamma_{\rho \sigma}^{\mu(1)}+\Gamma_{\rho \sigma}^{\mu(2)} / 2$ and $\hat{\Gamma}_{\rho \sigma}^{\mu}=\hat{\Gamma}_{\rho \sigma}^{\mu(0)}+\hat{\Gamma}_{\rho \sigma}^{\mu(1)}+\hat{\Gamma}_{\rho \sigma}^{\mu(2)} / 2$ in comoving coordinates, we obtain

$$
\begin{array}{rlrl}
\Gamma_{00}^{0(0)}=\mathcal{H}, & \Gamma_{0 i}^{0(0)}=0, & \Gamma_{00}^{i(0)}=0, \\
\Gamma_{00}^{i(0)}=0, & \Gamma_{j 0}^{i(0)}=\mathcal{H} \delta_{j}^{i}, & \Gamma_{j k}^{i(0)}=0, \quad \hat{\Gamma}_{\rho \sigma}^{\mu(0)}=0, \\
\Gamma_{00}^{0(1)}=\hat{\Gamma}_{00}^{0(1)} & \Gamma_{0 i}^{0(1)}=\hat{\Gamma}_{0 i}^{0(1)}-\mathcal{H} B_{i}^{(1)} \\
\Gamma_{i j}^{0(1)}=\hat{\Gamma}_{i j}^{0(1)}+\mathcal{H}\left(-2 A^{(1)} \delta_{i j}+h_{i j}^{(1)}\right) & \Gamma_{00}^{i(1)}=\hat{\Gamma}_{00}^{i(1)}-\mathcal{H} B^{i(1)} \\
\Gamma_{j 0}^{i(1)}=\hat{\Gamma}_{j 0}^{i(1)} & \Gamma_{j k}^{i(1)}=\hat{\Gamma}_{j k}^{i(1)}+\mathcal{H} B^{i(1)} \delta_{j k}, \\
\hat{\Gamma}_{00}^{0(1)}=A^{(1)^{\prime}}, & \hat{\Gamma}_{0 i}^{0(1)}=\partial_{i} A^{(1)}, \\
\hat{\Gamma}_{i j}^{0(1)}=\frac{1}{2} \partial_{i} B_{j}^{(1)}+\frac{1}{2} \partial_{j} B_{i}^{(1)}+\frac{1}{2} h_{i j}^{(1)^{\prime}}, & \hat{\Gamma}_{00}^{i(1)}=\partial^{i} A^{(1)}-B^{i(1)^{\prime}}, \\
\hat{\Gamma}_{j 0}^{i(1)}=\frac{1}{2} \partial^{i} B_{j}^{(1)}-\frac{1}{2} \partial_{j} B^{i(1)}+\frac{1}{2} h_{j}^{i(1)^{\prime}}, & \hat{\Gamma}_{j k}^{i(1)}=\frac{1}{2} \partial_{j} h_{k}^{i(1)}+\frac{1}{2} \partial_{k} h_{j}^{i(1)}-\frac{1}{2} \partial^{i} h_{j k}^{(1)},
\end{array}
$$




$$
\begin{aligned}
& \frac{1}{2} \Gamma_{00}^{0(2)}= \frac{1}{2} \hat{\Gamma}_{00}^{0(2)}+\mathcal{H} B_{k}^{(1)} B^{k(1)} \\
& \frac{1}{2} \Gamma_{0 i}^{0(2)}= \frac{1}{2} \hat{\Gamma}_{0 i}^{0(2)}-\frac{\mathcal{H}}{2} B_{i}^{(2)}+2 \mathcal{H} A^{(1)} B_{i}^{(1)} \\
& \frac{1}{2} \Gamma_{00}^{i(2)}= \frac{1}{2} \hat{\Gamma}_{00}^{i(2)}-\frac{\mathcal{H}}{2} B^{i(2)}+\mathcal{H} B_{k}^{(1)} h^{i k(1)} \\
& \frac{1}{2} \Gamma_{j 0}^{i(2)}= \frac{1}{2} \hat{\Gamma}_{j 0}^{i(2)}-\mathcal{H} B^{i(1)} B_{j}^{(1)} \\
& \frac{1}{2} \Gamma_{i j}^{0(2)}= \frac{1}{2} \hat{\Gamma}_{i j}^{0(2)}+\mathcal{H}\left\{\left[-A^{(2)}+4\left(A^{(1)}\right)^{2}-B_{k}^{(1)} B^{k(1)}\right] \delta_{i j}+\frac{1}{2} h_{i j}^{(2)}-2 A^{(1)} h_{i j}^{(1)}\right\} \\
& \frac{1}{2} \Gamma_{j k}^{i(2)}= \frac{1}{2} \hat{\Gamma}_{j k}^{i(2)}+\mathcal{H}\left[\left(\frac{1}{2} B^{i(2)}-2 A^{(1)} B^{i(1)}-B_{l}^{(1)} h^{i l(1)}\right) \delta_{j k}-B^{i(1)} h_{j k}^{(1)}\right] \\
& \frac{1}{2} \hat{\Gamma}_{00}^{0(2)}=+\frac{1}{2} A^{2)^{\prime}}-2 A^{(1)} A^{(1)^{\prime}}+B_{k}^{(1)} B^{k(1)^{\prime}}-\partial_{k} A^{(1)} B^{k(1)} \\
& \frac{1}{2} \hat{\Gamma}_{0 i}^{0(2)}= \frac{1}{2} \partial_{i} A^{(2)}-2 A^{(1)} \partial_{i} A^{(1)}-\frac{1}{2} B^{k(1)} h_{i k}^{(1)^{\prime}}+\frac{1}{2} B^{k(1)}\left(\partial_{i} B_{k}^{(1)}-\partial_{k} B_{i}^{(1)}\right) \\
& \frac{1}{2} \hat{\Gamma}_{00}^{i(2)}= \frac{1}{2} \partial^{i} A^{(2)}-\frac{1}{2} B^{i(2)^{\prime}}+A^{(1)^{\prime}} B^{i(1)}+B_{k}^{(1)^{\prime}} h^{i k(1)}-\partial_{k} A^{(1)} h^{i k(1)}, \\
& \frac{1}{2} \hat{\Gamma}_{j 0}^{i(2)}= \frac{1}{4} \partial^{i} B_{j}^{(2)}-\frac{1}{4} \partial_{j} B^{i(2)}+\frac{1}{4} h_{j}^{i(2)^{\prime}}-\frac{1}{2} h^{i k(1)} h_{j k}^{(1)^{\prime}}+\partial_{j} A^{(1)} B^{i(1)}-\frac{1}{2} h^{i k(1)}\left(\partial_{k} B_{j}^{(1)}-\frac{1}{2} \partial_{j} B_{k}^{(1)}\right) \\
& \frac{1}{2} \hat{\Gamma}_{i j}^{0(2)}= \frac{1}{4} \partial_{i} B_{j}^{(2)}+\frac{1}{4} \partial_{j} B_{i}^{(2)}+\frac{1}{4} h_{i j}^{(2)^{\prime}}-A^{(1)}\left(\partial_{i} B_{j}^{(1)}+\partial_{j} B_{i}^{(1)}\right)-A^{(1)} h_{i j}^{(1)^{\prime}}-\frac{1}{2} B^{k(1)}\left(\partial_{i} h_{j k}^{(1)}+\partial_{j} h_{i k}^{(1)}-\partial_{k} h_{i j}^{(1)}\right) \\
& \frac{1}{2} \hat{\Gamma}_{j k}^{i(2)}= \frac{1}{4} \partial_{j} h_{k}^{i(2)}+\frac{1}{4} \partial_{k} h_{j}^{i(2)}-\frac{1}{4} \partial^{i} h_{j k}^{(2)}+\frac{1}{2} B^{i(1)}\left(\partial_{j} B_{k}^{(1)}+\partial_{k} B_{j}^{(1)}\right)+\frac{1}{2} B^{i(1)} h_{j k}^{(1)}{ }^{\prime} \\
&-\frac{1}{2} h^{i l(1)}\left(\partial_{j} h_{k l}^{(1)}+\partial_{k} h_{j l}^{(1)}-\partial_{l} h_{j k}^{(1)}\right) \\
&
\end{aligned}
$$

For four-velocity $u^{\mu}\left(g_{\mu \nu} u^{\mu} u^{\nu}=-1\right)$, we find

$$
\begin{aligned}
& u_{0}=-a\left[1+A^{(1)}+\frac{1}{2} A^{(2)}-\frac{1}{2}\left(A^{(1)}\right)^{2}+\frac{1}{2} v_{k}^{(1)} v^{k(1)}\right] \\
& u_{i}=a\left[v_{i}^{(1)}-B_{i}^{(1)}+\frac{1}{2}\left(v_{i}^{(2)}-B_{i}^{(2)}\right)+A^{(1)} B_{i}^{(1)}+h_{i k}^{(1)} v^{k(1)}\right] \\
& u^{0}=\frac{1}{a}\left[1-A^{(1)}-\frac{1}{2} A^{(2)}+\frac{3}{2}\left(A^{(1)}\right)^{2}+\frac{1}{2} v_{k}^{(1)} v^{k(1)}-v_{k}^{(1)} B^{k(1)}\right], \\
& u^{i}=\frac{1}{a}\left(v^{i(1)}+\frac{1}{2} v^{i(2)}\right) .
\end{aligned}
$$

From Eqs. 23 and A5 we obtain all components of $\Lambda_{\hat{0} \mu}^{(n)}$ and $E_{\hat{0} \mu}^{(n)}$. From Eq. 23 , we have

$$
u_{\mu}=\Lambda_{\hat{0} \mu}=a E_{\hat{0} \mu} \quad \text { and } \quad u^{\mu}=\Lambda_{\hat{0}}^{\mu}=E_{\hat{0}}^{\mu} / a,
$$

and, using Eq. 90 we can deduce all components of $\Lambda_{\hat{a} \mu}^{(n)}$ and $E_{\hat{a} \mu}^{(n)}$. We summarize as follows: 


$$
\begin{array}{ll}
\Lambda_{\hat{0} 0}^{(1)}=a E_{\hat{0} 0}^{(1)}=-a A^{(1)}, & \Lambda_{\hat{0} i}^{(1)}=a E_{\hat{0} i}^{(1)}=a\left(v_{i}^{(1)}-B_{i}^{(1)}\right), \\
\Lambda_{\hat{a} 0}^{(1)}=a E_{\hat{a} 0}^{(1)}=-a v_{\hat{a}}^{(1)}, & \Lambda_{\hat{a} i}^{(1)}=a E_{\hat{a} i}^{(1)}=\frac{1}{2} a h_{\hat{a} i}^{(1)}, \\
\frac{1}{2} \Lambda_{\hat{0} 0}^{(2)}=\frac{1}{2} a E_{\hat{0} 0}^{(2)}=a\left[-\frac{1}{2} A^{(2)}+\frac{1}{2}\left(A^{(1)}\right)^{2}-\frac{1}{2} v_{k}^{(1)} v^{k(1)}\right], & \\
\frac{1}{2} \Lambda_{\hat{0} i}^{(2)}=\frac{1}{2} a E_{\hat{0} i}^{(2)}=a\left[\frac{1}{2}\left(v_{i}^{(2)}-B_{i}^{(2)}\right)+A^{(1)} B_{i}^{(1)}+h_{i k}^{(1)} v^{k(1)}\right], & \\
\frac{1}{2} \Lambda_{\hat{a} 0}^{(2)}=\frac{1}{2} a E_{\hat{a} 0}^{(2)}=a\left[-\frac{1}{2} v_{\hat{a}}^{(2)}-A^{(1)} v_{\hat{a}}^{(1)}-\frac{1}{2} v^{k(1)} h_{\hat{a} k}^{(1)}\right] \\
\frac{1}{2} \Lambda_{\hat{a} i}^{(2)}=\frac{1}{2} a E_{\hat{a} i}^{(2)}=a\left[\frac{1}{4} h_{\hat{a} j}^{(2)}+\frac{1}{2}\left(v_{i}^{(1)}-B_{i}^{(1)}\right)\left(v_{\hat{a}}^{(1)}-B_{\hat{a}}^{(1)}\right)-\frac{1}{8} h_{j}^{k(1)} h_{\hat{a} k}^{(1)}\right] .
\end{array}
$$

The four-vector $\mathcal{E}_{m}^{\nu}$ defined in Eq. $(160)$ can be nonzero. For the background, first- and second-order perturbations we obtain

$$
\begin{aligned}
\mathcal{E}_{m}^{0(0)}= & \frac{1}{a^{2}} \rho_{m}^{(0) \prime}+\frac{3}{a^{2}} \mathcal{H} \rho_{m}^{(0)} \\
\mathcal{E}_{m}^{i(0)}= & 0 \\
\mathcal{E}_{m}^{0(1)}= & \frac{1}{a^{2}} \rho_{m}^{(0)}\left(\delta_{m}^{(1) \prime}+\partial_{i} v^{i(1)}+\frac{1}{2} h_{i}^{i(1) \prime}\right)+\mathcal{E}_{m}^{0(0)}\left(-2 A^{(1)}+\delta_{m}^{(1)}\right) \\
\mathcal{E}_{m}^{i(1)}= & \frac{1}{a^{2}} \rho_{m}^{(0)}\left[\left(v^{i(1)}-B^{i(1)}\right)^{\prime}+\mathcal{H}\left(v^{i(1)}-B^{i(1)}\right)+\partial^{i} A^{(1)}\right]+\mathcal{E}_{m}^{0(0)} v^{i(1)} \\
\frac{1}{2} \mathcal{E}_{m}^{0(2)}= & \frac{1}{a^{2}} \rho_{m}^{(0)}\left[\frac{1}{2} \delta_{m}^{(2) \prime}+\frac{1}{2} \partial_{i} v^{i(2)}+\frac{1}{4} h_{i}^{i(2) \prime}-\mathcal{H}\left(v^{i(1)}-B^{i(1)}\right)\left(v_{i}^{(1)}-B_{i}^{(1)}\right)+B_{i}^{(1)} \partial^{i} A^{(1)}+\left(A^{(1)}+\delta_{m}^{(1)}\right) \partial_{i} v^{i(1)}\right. \\
& \left.+v^{i(1)} \partial_{i} \delta_{m}^{(1)}+\frac{1}{2} \delta_{m}^{(1)} h_{i}^{i(1) \prime}+\frac{1}{2} v^{j(1)} \partial_{j} h_{i}^{i(1)}-\frac{1}{2} h^{i j(1)} h_{i j}^{(1) \prime}\right]+\mathcal{E}_{m}^{0(0)}\left(-A^{(2)}+\frac{1}{2} \delta_{m}^{(2)}-v^{i(1)} v_{i}^{(1)}\right) \\
& +2\left(v^{i(1)}-B^{i(1)}\right) \mathcal{E}_{m}^{i(1)}-2 A^{(1)} \mathcal{E}_{m}^{0(1)}, \\
\frac{1}{2} \mathcal{E}_{m}^{i(2)}= & \frac{1}{a^{2}} \rho_{m}^{(0)}\left[\left(\frac{1}{2} v^{i(2)}-\frac{1}{2} B^{i(2)}\right) \prime+\mathcal{H}\left(\frac{1}{2} v^{i(2)}-\frac{1}{2} B^{i(2)}\right)+\frac{1}{2} \partial^{i} A^{(2)}-v^{i(1)} \partial_{j} v^{j(1)}+A^{(1)} B^{i(1) \prime}+\mathcal{H} A^{(1)} B^{i(1)}\right. \\
& +A^{(1) \prime} B^{i(1)}+\mathcal{H} B_{k}^{(1)} h^{i k(1)}+B_{k}^{(1) \prime} h^{i k(1)}+v^{k(1)} h_{k}^{i(1) \prime}-A^{(1)} \partial^{i} A^{(1)}+\partial^{i} B_{k}^{(1)} v^{k(1)}-v^{k(1)} \partial_{k} B^{i(1)} \\
& \left.-\partial_{k} A^{(1)} h^{i k(1)}\right]+\mathcal{E}_{m}^{0(0)}\left(\frac{1}{2} v^{i(2)}-\delta_{m}^{(1)} v^{i(1)}+2 A^{(1)} v^{i(1)}\right)+\mathcal{E}_{m}^{0(1)} v^{i(1)}+\mathcal{E}_{m}^{i(1)}\left(\delta_{m}^{(1)}-A^{(1)}\right) .
\end{aligned}
$$

Here $\delta_{m}^{(1)}=\rho_{m}^{(1)} / \rho_{m}^{(0)}-1$ is the CDM fractional overdensity. If $\mathcal{E}_{m}^{\nu}$ is evaluated in redshift-space, then $\rho_{m}^{(0)}=\bar{\rho}_{m}$, $a\left(\bar{x}^{0}\right)=\bar{a}$ and

$$
\mathcal{E}_{m}^{0(0)}=\frac{\mathcal{H}}{\bar{a}^{2}} \bar{\rho}_{m} b_{m}
$$

where $b_{m}=\mathrm{d}\left(a^{3} \bar{\rho}_{m}\right) / \mathrm{d} \ln \bar{a}$. 
$\mathcal{E}_{m}^{\|(1)}$ and $\mathcal{E}_{m}^{\|(2)}$, evaluated at $\bar{x}^{\mu}$, are given by

$$
\begin{aligned}
\mathcal{E}_{m}^{\|(1)}= & \frac{1}{\bar{a}^{2}} \bar{\rho}_{m}\left[\frac{\mathrm{d}}{\mathrm{d} \bar{\chi}}\left(A^{(1)}-v_{\|}^{(1)}\right)+A^{(1) \prime}-B_{\|}^{(1) \prime}+\partial_{\|} v_{\|}^{(1)}+\mathcal{H}\left(v_{\|}^{(1)}-B_{\|}^{(1)}\right)\right]+\frac{\mathcal{H}}{\bar{a}^{2}} \bar{\rho}_{m} b_{m} v_{\|}^{(1)}, \\
\frac{1}{2} \mathcal{E}_{m}^{\|(2)}= & \frac{1}{\bar{a}^{2}} \bar{\rho}_{m}\left[\frac{\mathrm{d}}{\mathrm{d} \bar{\chi}}\left(\frac{1}{2} A^{(2)}-\frac{1}{2} v_{\|}^{(2)}\right)+\frac{1}{2} A^{(2) \prime}-\frac{1}{2} B_{\|}^{(2) \prime}+\frac{1}{2} \partial_{\|} v_{\|}^{(2)}+\mathcal{H}\left(\frac{1}{2} v_{\|}^{(2)}-\frac{1}{2} B_{\|}^{(2)}\right)-v_{\|}^{(1)} \partial_{\|} v_{\|}^{(1)}\right. \\
& -\frac{2}{\bar{\chi}}\left(v_{\|}^{(1)}\right)^{2}-v_{\|}^{(1)} \partial_{\perp j} v_{\perp}^{j(1)}+A^{(1)} B_{\|}^{(1) \prime}+A^{(1) \prime} B_{\|}^{(1)}+\mathcal{H} A^{(1)} B_{\|}^{(1)}+v_{\|}^{(1)} h_{\|}^{(1) \prime}+\mathcal{H} B_{\|}^{(1)} h_{\|}^{(1)}+B_{\|}^{(1) \prime} h_{\|}^{(1)} \\
& -A^{(1)} \partial_{\|} A^{(1)}-\partial_{\|} A^{(1)} h_{\|}^{(1)}+v_{\perp k}^{(1)} \partial_{\|} B_{\perp}^{k(1)}-v_{\perp}^{j(1)} \partial_{\perp j} B_{\|}^{(1)}+\frac{1}{\chi} v_{\perp}^{j(1)} B_{\perp j}^{(1)}+\mathcal{H} B_{k}^{(1)} \mathcal{P}_{j}^{k} h^{i j(1)} n_{i} \\
& \left.+v_{k}^{(1)} \mathcal{P}_{j}^{k} h^{i j(1) \prime} n_{i}+B_{k}^{(1) \prime} \mathcal{P}_{j}^{k} h^{i j(1)} n_{i}-\partial_{k} A^{(1)} \mathcal{P}_{j}^{k} h^{i j(1)} n_{i}\right]+\frac{\mathcal{H}}{\bar{a}^{2}} \bar{\rho}_{m} b_{m}\left(\frac{1}{2} v_{\|}^{(2)}-\delta_{m}^{(1)} v_{\|}^{(1)}+2 A^{(1)} v_{\|}^{(1)}\right) \\
& +\mathcal{E}_{m}^{0(1)} v_{\|}^{(1)}+\mathcal{E}_{m}^{\|(1)}\left(\delta_{m}^{(1)}-A^{(1)}\right) .
\end{aligned}
$$

For the weak lensing shear and rotation:

$$
\begin{aligned}
& \partial_{\perp i} \Delta x_{\perp j}^{(1)}=-\mathcal{P}_{i j}\left(B_{\| o}^{(1)}-v_{\| o}^{(1)}\right)+\frac{1}{2} \mathcal{P}_{i}^{m} \mathcal{P}_{j}^{n} h_{m n o}^{(1)}-\frac{1}{2} \mathcal{P}_{i j} h_{\| o}^{(1)}-\left(B_{\perp i o}^{(1)}-v_{\perp i o}^{(1)}\right) n_{j}-\frac{1}{2} \mathcal{P}_{i p} n^{k} h_{k o}^{p(1)} n_{j} \\
& +\int_{0}^{\bar{\chi}} \mathrm{d} \tilde{\chi}\left[\frac{1}{\tilde{\chi}} \mathcal{P}_{i j} B_{\|}^{(1)}-\mathcal{P}_{j p} \tilde{\partial}_{\perp i} B^{p(1)}-\frac{1}{\tilde{\chi}} \mathcal{P}_{i}^{p} \mathcal{P}_{j q} h_{p}^{q(1)}+\frac{1}{\tilde{\chi}} \mathcal{P}_{i j} h_{\|}^{(1)}-n^{p} \mathcal{P}_{j q} \tilde{\partial}_{\perp i} h_{p}^{q(1)}\right. \\
& \left.+\frac{1}{\tilde{\chi}}\left(B_{\perp i}^{(1)}+\mathcal{P}_{i n} n^{m} h_{m}^{n(1)}\right) n_{j}\right]+\int_{0}^{\bar{\chi}} \mathrm{d} \tilde{\chi}(\bar{\chi}-\tilde{\chi}) \frac{\tilde{\chi}}{\bar{\chi}} \tilde{\partial}_{\perp i} \tilde{\partial}_{\perp j}\left(A^{(1)}-B_{\|}^{(1)}-\frac{1}{2} h_{\|}^{(1)}\right), \\
& \gamma_{i j}^{(1)}=\mathcal{P}_{i j}\left(B_{\| o}^{(1)}-v_{\| o}^{(1)}\right)-\frac{1}{2} \mathcal{P}_{(i}^{m} \mathcal{P}_{j)}^{n} h_{m n o}^{(1)}+\frac{1}{2} \mathcal{P}_{i j} h_{\| o}^{(1)}+n_{(j}\left(B_{\perp i) o}^{(1)}-v_{\perp i) o}^{(1)}\right)+\frac{1}{2} \mathcal{P}_{p(i} n_{j)} n^{k} h_{k o}^{p(1)} \\
& -\int_{0}^{\bar{\chi}} \mathrm{d} \tilde{\chi}\left[\frac{1}{\tilde{\chi}} \mathcal{P}_{i j} B_{\|}^{(1)}-\mathcal{P}_{p(j} \tilde{\partial}_{\perp i)} B^{p(1)}-\frac{1}{\tilde{\chi}} \mathcal{P}_{(i}^{p} \mathcal{P}_{j) q} h_{p}^{q(1)}+\frac{1}{\tilde{\chi}} \mathcal{P}_{i j} h_{\|}^{(1)}-n^{p} \mathcal{P}_{q(j} \tilde{\partial}_{\perp i)} h_{p}^{q(1)}\right. \\
& \left.+\frac{1}{\tilde{\chi}}\left(B_{\perp(i}^{(1)}+n^{m} h_{m}^{n(1)} \mathcal{P}_{n(i}\right) n_{j)}\right]-\int_{0}^{\bar{\chi}} \mathrm{d} \tilde{\chi}(\bar{\chi}-\tilde{\chi}) \frac{\tilde{\chi}}{\bar{\chi}} \tilde{\partial}_{\perp(i} \tilde{\partial}_{\perp j)}\left(A^{(1)}-B_{\|}^{(1)}-\frac{1}{2} h_{\|}^{(1)}\right)-\mathcal{P}_{i j} \kappa^{(1)}, \\
& \vartheta_{i j}^{(1)} \vartheta^{i j(1)}=\frac{1}{2} B_{\perp i o}^{(1)} B_{\perp o}^{i(1)}-B_{\perp i o}^{(1)} v_{\perp o}^{i(1)}+\frac{1}{2} v_{\perp i o}^{(1)} v_{\perp o}^{i(1)}+\frac{1}{2}\left(B_{\perp i o}^{(1)}-v_{\perp i o}^{(1)}\right) n^{k} h_{k o}^{i(1)}+\frac{1}{8} n^{m} \mathcal{P}_{n}^{j} h_{m}^{n(1)} n^{k} h_{j k}^{(1)} \\
& -\left(B_{\perp i o}^{(1)}-v_{\perp i o}^{(1)}+\frac{1}{2} \mathcal{P}_{i p} n^{k} h_{k o}^{p(1)}\right) \int_{0}^{\bar{\chi}} \mathrm{d} \tilde{\chi}\left[\frac{1}{\tilde{\chi}}\left(B_{\perp}^{i(1)}+\mathcal{P}_{l}^{i} n^{m} h_{m}^{l(1)}\right)+(\bar{\chi}-\tilde{\chi}) \frac{1}{\bar{\chi}} \tilde{\partial}_{\perp}^{i}\left(A^{(1)}-B_{\|}^{(1)}-\frac{1}{2} h_{\|}^{(1)}\right)\right] \\
& +\int_{0}^{\bar{\chi}} \mathrm{d} \tilde{\chi}\left[\mathcal{P}_{q[i} \tilde{\partial}_{\perp j]} B^{q(1)}+n^{k} \mathcal{P}_{q[i} \tilde{\partial}_{\perp j]} h_{k}^{q(1)}\right] \int_{0}^{\bar{\chi}} \mathrm{d} \tilde{\chi}\left[\mathcal{P}_{p}^{[i} \tilde{\partial}_{\perp}^{j]} B^{p(1)}+n^{m} \mathcal{P}_{p}^{[i} \tilde{\partial}_{\perp}^{j]} h_{m}^{p(1)}\right] \\
& +\frac{1}{2} \int_{0}^{\bar{\chi}} \mathrm{d} \tilde{\chi}\left[\frac{1}{\tilde{\chi}}\left(B_{\perp i}^{(1)}+\mathcal{P}_{i n} n^{m} h_{m}^{n(1)}\right)+(\bar{\chi}-\tilde{\chi}) \frac{1}{\bar{\chi}} \tilde{\partial}_{\perp i}\left(A^{(1)}-B_{\|}^{(1)}-\frac{1}{2} h_{\|}^{(1)}\right)\right] \\
& \times \int_{0}^{\bar{\chi}} \mathrm{d} \tilde{\chi}\left[\frac{1}{\tilde{\chi}}\left(B_{\perp}^{i(1)}+\mathcal{P}_{p}^{i} n^{q} h_{q}^{p(1)}\right)+(\bar{\chi}-\tilde{\chi}) \frac{1}{\bar{\chi}} \tilde{\partial}_{\perp}^{i}\left(A^{(1)}-B_{\|}^{(1)}-\frac{1}{2} h_{\|}^{(1)}\right)\right] .
\end{aligned}
$$


If we assume that galaxy velocities follow the matter velocity field,

$$
\begin{aligned}
& \Delta_{g}^{(2)}=\delta_{g}^{(2)}+b_{e} \Delta \ln a^{(2)}+\partial_{\|} \Delta x_{\|}^{(2)}+\frac{2}{\bar{\chi}} \Delta x_{\|}^{(2)}-2 \kappa^{(2)}+A^{(2)}+v_{\|}^{(2)}+\frac{1}{2} h_{i}^{i(2)}+\left(\Delta_{g}^{(1)}\right)^{2}-\left(A^{(1)}\right)^{2} \\
& +A^{(1)} h_{\|}^{(1)}-\left(v_{\|}^{(1)}\right)^{2}+\left(B_{\|}^{(1)}\right)^{2}-\frac{1}{2} h_{i}^{k(1)} h_{k}^{i(1)}-\frac{1}{4}\left(h_{\|}^{(1)}\right)^{2}+2 A^{(1)} v_{\|}^{(1)}-\frac{1}{\mathcal{H}^{2}}\left(\partial_{\|} v_{\|}^{(1)}\right)^{2}+\frac{1}{\mathcal{H}} A^{(1)} h_{\|}^{(1) \prime} \\
& -\frac{1}{4 \mathcal{H}^{2}}\left(h_{\|}^{(1) \prime}\right)^{2}-\frac{1}{\mathcal{H}} v_{\|}^{(1)} h_{\|}^{(1) \prime}-\frac{1}{2 \mathcal{H}} h_{\|}^{(1)} h_{\|}^{(1) \prime}+\frac{2}{\mathcal{H}} A^{(1)} \partial_{\|} v_{\|}^{(1)}-\frac{2}{\mathcal{H}} v_{\|}^{(1)} \partial_{\|} v_{\|}^{(1)}-\frac{1}{\mathcal{H}} h_{\|}^{(1)} \partial_{\|} v_{\|}^{(1)}-\frac{1}{\mathcal{H}^{2}} h_{\|}^{(1) \prime} \partial_{\|} v_{\|}^{(1)} \\
& -2\left|\gamma^{(1)}\right|^{2}-2\left(\kappa^{(1)}\right)^{2}+\vartheta_{i j}^{(1)} \vartheta^{i j(1)}+B_{\perp i}^{(1)} B_{\perp}^{i(1)}+v_{\perp i}^{(1)} v_{\perp}^{i(1)}-2 v_{\perp i}^{(1)} B_{\perp}^{i(1)}-\left(\delta_{g}^{(1)}\right)^{2}+2 v_{\perp}^{i(1)} \partial_{\perp i} T^{(1)} \\
& +\frac{2}{\mathcal{H}}\left(-\partial_{\|} A^{(1)}+B_{\|}^{(1) \prime}-\partial_{\|} v_{\|}^{(1)}-\mathcal{H} v_{\|}^{(1)}+\mathcal{H} B_{\|}^{(1)}\right) \Delta \ln a^{(1)}-\frac{1}{\mathcal{H}} \frac{\mathrm{d}}{\mathrm{d} \bar{\chi}}\left(h_{i}^{i(1)}+2 \delta_{g}^{(1)}\right) \Delta \ln a^{(1)}-\frac{4}{\bar{\chi}^{2} \mathcal{H}} \Delta \ln a^{(1)} T^{(1)} \\
& +2 \frac{\mathcal{H}^{\prime}}{\mathcal{H}^{2}}\left(A^{(1)}-v_{\|}^{(1)}-\frac{1}{2} h_{\|}^{(1)}-\frac{1}{\mathcal{H}} \partial_{\|} v_{\|}^{(1)}-\frac{1}{2 \mathcal{H}} h_{\|}^{(1) \prime}\right) \Delta \ln a^{(1)}-\partial_{\|}\left(2 v_{\|}^{(1)}+h_{i}^{i(1)}+2 \delta_{g}^{(1)}\right) T^{(1)}-\frac{4}{\bar{\chi} \mathcal{H}} \Delta \ln a^{(1)} \kappa^{(1)} \\
& -\frac{4}{\bar{\chi}} T^{(1)} \kappa^{(1)}+\left[-b_{e}+\frac{\mathrm{d} \ln b_{e}}{\mathrm{~d} \ln \bar{a}}-\left(\frac{\mathcal{H}^{\prime}}{\mathcal{H}^{2}}\right)^{2}-\frac{2}{\bar{\chi}^{2} \mathcal{H}^{2}}\right]\left(\Delta \ln a^{(1)}\right)^{2}-\frac{2}{\bar{\chi}^{2}}\left(T^{(1)}\right)^{2}+2\left[-\left(B_{\perp}^{i(1)}+n^{k} h_{k}^{j(1)} \mathcal{P}_{j}^{i}\right)+2 S_{\perp}^{i(1)}\right] \\
& \times \partial_{\perp i}\left(\frac{1}{\mathcal{H}} \Delta \ln a^{(1)}+T^{(1)}\right)-\left[-\frac{2}{\bar{\chi}}\left(B_{\perp}^{i(1)}+n^{k} h_{k}^{j(1)} \mathcal{P}_{j}^{i}\right)+\frac{4}{\bar{\chi}} S_{\perp}^{i(1)}+\partial_{\perp}^{i}\left(2 v_{\|}^{(1)}+h_{l}^{l(1)}+2 \delta_{g}^{(1)}\right)\right] \\
& \times \int_{0}^{\bar{\chi}} \mathrm{d} \tilde{\chi}\left[\frac{\bar{\chi}}{\tilde{\chi}}\left(B_{\perp i}^{(1)}+n^{k} h_{k}^{j(1)} \mathcal{P}_{i j}\right)+(\bar{\chi}-\tilde{\chi}) \tilde{\partial}_{\perp i}\left(A^{(1)}-B_{\|}^{(1)}-\frac{1}{2} h_{\|}^{(1)}\right)\right] \\
& +2\left(B_{\perp i o}^{(1)}-v_{\perp i o}^{(1)}+\frac{1}{2} n^{k} h_{k o}^{j(1)} \mathcal{P}_{i j}\right)\left(B_{\perp o}^{i(1)}-v_{\perp o}^{i(1)}+\frac{1}{2} n^{k} h_{k o}^{j(1)} \mathcal{P}_{j}^{i}\right) \\
& +\left(B_{\perp i o}^{(1)}-v_{\perp i o}^{(1)}+\frac{1}{2} n^{k} h_{k o}^{j(1)} \mathcal{P}_{i j}\right)\left\{-2\left(B_{\perp}^{i(1)}+n^{m} h_{m}^{l(1)} \mathcal{P}_{l}^{i}\right)+4 S_{\perp}^{i(1)}+\bar{\chi} \partial_{\perp}^{i}\left(2 v_{\|}^{(1)}+h_{l}^{l(1)}+2 \delta_{g}^{(1)}\right)\right. \\
& \left.+2 \partial_{\perp i}\left(\frac{1}{\mathcal{H}} \Delta \ln a^{(1)}+T^{(1)}\right)-2 \frac{1}{\bar{\chi}} \int_{0}^{\bar{\chi}} \mathrm{d} \tilde{\chi}\left[\frac{\bar{\chi}}{\tilde{\chi}}\left(B_{\perp}^{i(1)}+n^{k} h_{k}^{j(1)} \mathcal{P}_{j}^{i}\right)+(\bar{\chi}-\tilde{\chi}) \tilde{\partial}_{\perp}^{i}\left(A^{(1)}-B_{\|}^{(1)}-\frac{1}{2} h_{\|}^{(1)}\right)\right]\right\} \\
& -\left(\frac{\bar{a}^{2}}{\bar{\rho}_{m} \mathcal{H}} \mathcal{E}_{m}^{\|(1)}-b_{m} v_{\|}^{(1)}\right)^{2}-2\left[A^{(1)}-v_{\|}^{(1)}-\frac{1}{2} h_{\|}^{(1)}-\frac{1}{\mathcal{H}} \partial_{\|} v_{\|}^{(1)}-\frac{1}{2 \mathcal{H}} h_{\|}^{(1) \prime}-\left(1+\frac{\mathcal{H}^{\prime}}{\mathcal{H}^{2}}\right) \Delta \ln a^{(1)}\right] \\
& \times\left(\frac{\bar{a}^{2}}{\bar{\rho}_{m} \mathcal{H}} \mathcal{E}_{m}^{\|(1)}-b_{m} v_{\|}^{(1)}\right) \text {. }
\end{aligned}
$$

\section{Appendix B: Perturbation terms in Poisson Gauge}

From Eq. 175, the perturbations of $g_{\mu \nu}$ and $g^{\mu \nu}$ are

$$
\begin{array}{ll}
g_{00}=-a^{2}\left(1+2 \Phi^{(1)}+\Phi^{(2)}\right), & g^{00}=-a^{-2}\left[1-2 \Phi^{(1)}-\Phi^{(2)}+4\left(\Phi^{(1)}\right)^{2}\right], \\
g_{0 i}=a^{2} \omega_{i}^{(2)}, & g^{0 i}=a^{-2} \omega^{i(2)}, \\
g_{i j}=a^{2}\left(\delta_{i j}-2 \delta_{i j} \Psi^{(1)}-\delta_{i j} \Psi^{(2)}+\hat{h}_{i j}^{(2)} / 2\right), & g^{i j}=a^{-2}\left[\delta^{i j}+2 \delta^{i j} \Psi^{(1)}+\delta^{i j} \Psi^{(2)}-\hat{h}^{i j(2)} / 2+4 \delta^{i j}\left(\Psi^{(1)}\right)^{2}\right],
\end{array}
$$

For four-velocity $u^{\mu}$, we find

$$
\begin{aligned}
& u_{0}=-a\left[1+\Phi^{(1)}+\frac{1}{2} \Phi^{(2)}-\frac{1}{2}\left(\Phi^{(1)}\right)^{2}+\frac{1}{2} v_{k}^{(1)} v^{k(1)}\right] \\
& u_{i}=a\left[v_{i}^{(1)}+\frac{1}{2}\left(v_{i}^{(2)}+2 \omega_{i}^{(2)}\right)-2 \Psi^{(1)} v_{i}^{(1)}\right] \\
& u^{0}=\frac{1}{a}\left[1-\Phi^{(1)}-\frac{1}{2} \Phi^{(2)}+\frac{3}{2}\left(\Phi^{(1)}\right)^{2}+\frac{1}{2} v_{k}^{(1)} v^{k(1)}\right] \\
& u^{i}=\frac{1}{a}\left(v^{i(1)}+\frac{1}{2} v^{i(2)}\right) .
\end{aligned}
$$


For the tetrad:

$$
\begin{array}{ll}
\Lambda_{\hat{0} 0}^{(1)}=a E_{\hat{0} 0}^{(1)}=-a \Phi^{(1)}, & \Lambda_{\hat{0} i}^{(1)}=a E_{\hat{0} i}^{(1)}=a v_{i}^{(1)}, \\
\Lambda_{\hat{a} 0}^{(1)}=a E_{\hat{a} 0}^{(1)}=-a v_{\hat{a}}^{(1)}, & \Lambda_{\hat{a} i}^{(1)}=a E_{\hat{a} i}^{(1)}=-a \delta_{\hat{a} i} \Psi^{(1)}, \\
\frac{1}{2} \Lambda_{\hat{0} 0}^{(2)}=\frac{1}{2} a E_{\hat{0} 0}^{(2)}=a\left[-\frac{1}{2} \Phi^{(2)}+\frac{1}{2}\left(\Phi^{(1)}\right)^{2}-\frac{1}{2} v_{k}^{(1)} v^{k(1)}\right], & \\
\frac{1}{2} \Lambda_{\hat{0} i}^{(2)}=\frac{1}{2} a E_{\hat{0} i}^{(2)}=a\left[\frac{1}{2}\left(v_{i}^{(2)}+2 \omega_{i}^{(2)}\right)-2 \Psi^{(1)} v_{i}^{(1)}\right], & \\
\frac{1}{2} \Lambda_{\hat{a} 0}^{(2)}=\frac{1}{2} a E_{\hat{a} 0}^{(2)}=a\left[-\frac{1}{2} v_{\hat{a}}^{(2)}-\Phi^{(1)} v_{\hat{a}}^{(1)}+v_{\hat{a}}^{(1)} \Psi^{(1)}\right], & \\
\frac{1}{2} \Lambda_{\hat{a} i}^{(2)}=\frac{1}{2} a E_{\hat{a} i}^{(2)}=a\left[-\frac{1}{2} \delta_{\hat{a} j} \Psi^{(2)}+\frac{1}{4} \hat{h}_{\hat{a} j}^{(2)}+\frac{1}{2} v_{i}^{(1)} v_{\hat{a}}^{(1)}-\frac{1}{2} \delta_{\hat{a} j}\left(\Phi^{(1)}\right)^{2}\right] .
\end{array}
$$

For the energy-momentum exchange four-vector $\mathcal{E}_{m}^{\nu}$, defined in Eq. 160):

$$
\begin{aligned}
\mathcal{E}_{m}^{0(1)}= & \frac{1}{a^{2}} \rho_{m}^{(0)}\left(\delta_{m}^{(1) \prime}+\partial_{i} v^{i(1)}-3 \Psi^{(1) \prime}\right)+\mathcal{E}_{m}^{0(0)}\left(-2 \Phi^{(1)}+\delta_{m}^{(1)}\right) \\
\mathcal{E}_{m}^{i(1)}= & \frac{1}{a^{2}} \rho_{m}^{(0)}\left[v^{i(1) \prime}+\mathcal{H} v^{i(1)}+\partial^{i} \Phi^{(1)}\right]+\mathcal{E}_{m}^{0(0)} v^{i(1)}, \\
\frac{1}{2} \mathcal{E}_{m}^{0(2)}= & \frac{1}{a^{2}} \rho_{m}^{(0)}\left[\frac{1}{2} \delta_{m}^{(2) \prime}+\frac{1}{2} \partial_{i} v^{i(2)}-\frac{3}{2} \Psi^{(2) \prime}+\frac{1}{4} \hat{h}_{i}^{i(2) \prime}-\mathcal{H} v^{i(1)} v_{i}^{(1)}+\left(\Phi^{(1)}+\delta_{m}^{(1)}\right) \partial_{i} v^{i(1)}\right. \\
& \left.+v^{i(1)} \partial_{i} \delta_{m}^{(1)}-3 \delta_{m}^{(1)} \Psi^{(1) \prime}-3 v^{j(1)} \partial_{j} \Psi^{(1)}-6 \Psi^{(1)} \Psi^{(1) \prime}\right]+\mathcal{E}_{m}^{0(0)}\left(-\Phi^{(2)}+\frac{1}{2} \delta_{m}^{(2)}-v^{i(1)} v_{i}^{(1)}\right) \\
& +2 v^{i(1)} \mathcal{E}_{m}^{i(1)}-2 \Phi^{(1)} \mathcal{E}_{m}^{0(1)}, \\
\frac{1}{2} \mathcal{E}_{m}^{i(2)}= & \frac{1}{a^{2}} \rho_{m}^{(0)}\left[\left(\frac{1}{2} v^{i(2)}+\omega^{i(2)}\right) \prime+\mathcal{H}\left(\frac{1}{2} v^{i(2)}+\omega^{i(2)}\right)+\frac{1}{2} \partial^{i} \Phi^{(2)}-v^{i(1)} \partial_{j} v^{j(1)}-2 v^{i(1)} \Psi^{(1) \prime}-\Phi^{(1)} \partial^{i} \Phi^{(1)}\right. \\
& \left.+2 \partial^{i} \Phi^{(1)} \Psi^{(1)}\right]+\mathcal{E}_{m}^{0(0)}\left(\frac{1}{2} v^{i(2)}-\delta_{m}^{(1)} v^{i(1)}+2 \Phi^{(1)} v^{i(1)}\right)+\mathcal{E}_{m}^{0(1)} v^{i(1)}+\mathcal{E}_{m}^{i(1)}\left(\delta_{m}^{(1)}-\Phi^{(1)}\right),
\end{aligned}
$$

or

$$
\begin{aligned}
\mathcal{E}_{m}^{\|(1)}= & \frac{1}{\bar{a}^{2}} \bar{\rho}_{m}\left[\frac{\mathrm{d}}{\mathrm{d} \bar{\chi}}\left(\Phi^{(1)}-v_{\|}^{(1)}\right)+\Phi^{(1) \prime}+\partial_{\|} v_{\|}^{(1)}+\mathcal{H} v_{\|}^{(1)}\right]+\frac{\mathcal{H}}{\bar{a}^{2}} \bar{\rho}_{m} b_{m} v_{\|}^{(1)} \\
\frac{1}{2} \mathcal{E}_{m}^{\|(2)}= & \frac{1}{\bar{a}^{2}} \bar{\rho}_{m}\left[\frac{\mathrm{d}}{\mathrm{d} \bar{\chi}}\left(\frac{1}{2} \Phi^{(2)}-\frac{1}{2} v_{\|}^{(2)}\right)+\frac{1}{2} \Phi^{(2) \prime}+\omega_{\|}^{(2) \prime}+\frac{1}{2} \partial_{\|} v_{\|}^{(2)}+\mathcal{H}\left(\frac{1}{2} v_{\|}^{(2)}+\omega_{\|}^{(2)}\right)-v_{\|}^{(1)} \partial_{\|} v_{\|}^{(1)}-\frac{2}{\bar{\chi}}\left(v_{\|}^{(1)}\right)^{2}\right. \\
& \left.-v_{\|}^{(1)} \partial_{\perp j} v_{\perp}^{j(1)}-2 v_{\|}^{(1)} \Psi^{(1) \prime}-\Phi^{(1)} \partial_{\|} \Phi^{(1)}+2 \partial_{\|} \Phi^{(1)} \Psi^{(1)}\right]+\frac{\mathcal{H}}{\bar{a}^{2}} \bar{\rho}_{m} b_{m}\left(\frac{1}{2} v_{\|}^{(2)}-\delta_{m}^{(1)} v_{\|}^{(1)}+2 \Phi^{(1)} v_{\|}^{(1)}\right) \\
& +\mathcal{E}_{m}^{(1)} v_{\|}^{(1)}+\mathcal{E}_{m}^{\|(1)}\left(\delta_{m}^{(1)}-\Phi^{(1)}\right) .
\end{aligned}
$$

For the weak lensing shear and rotation:

$$
\begin{aligned}
\partial_{\perp i} \Delta x_{\perp j}^{(1)}= & \mathcal{P}_{i j} v_{\| o}^{(1)}+v_{\perp i o}^{(1)} n_{j}+\int_{0}^{\bar{\chi}} \mathrm{d} \tilde{\chi}(\bar{\chi}-\tilde{\chi}) \frac{\tilde{\chi}}{\bar{\chi}} \tilde{\partial}_{\perp i} \tilde{\partial}_{\perp j}\left(\Phi^{(1)}+\Psi^{(1)}\right), \\
\gamma_{i j}= & -\mathcal{P}_{i j} v_{\| o}^{(1)}-n_{(j} v_{\perp i o o}^{(1)}-\int_{0}^{\bar{\chi}} \mathrm{d} \tilde{\chi}\left[(\bar{\chi}-\tilde{\chi}) \frac{\tilde{\chi}}{\bar{\chi}} \tilde{\partial}_{\perp(i} \tilde{\partial}_{\perp j)}\left(\Phi^{(1)}+\Psi^{(1)}\right)\right]-\mathcal{P}_{i j} \kappa^{(1)}, \\
\vartheta_{i j}^{(1)} \vartheta^{i j(1)}= & +\frac{1}{2} v_{\perp i o}^{(1)} v_{\perp o}^{i(1)}+\frac{1}{\bar{\chi}} v_{\perp i o}^{(1)} \int_{0}^{\bar{\chi}} \mathrm{d} \tilde{\chi}\left[(\bar{\chi}-\tilde{\chi}) \tilde{\partial}_{\perp}^{i}\left(\Phi^{(1)}+\Psi^{(1)}\right)\right] \\
& +\frac{1}{2 \bar{\chi}^{2}} \int_{0}^{\bar{\chi}} \mathrm{d} \tilde{\chi}\left[(\bar{\chi}-\tilde{\chi}) \tilde{\partial}_{\perp i}\left(\Phi^{(1)}+\Psi^{(1)}\right)\right] \int_{0}^{\bar{\chi}} \mathrm{d} \tilde{\chi}\left[(\bar{\chi}-\tilde{\chi}) \tilde{\partial}_{\perp}^{i}\left(\Phi^{(1)}+\Psi^{(1)}\right)\right] .
\end{aligned}
$$


Assuming that galaxy velocities follow the matter velocity field, we find

$$
\begin{aligned}
& \Delta_{g}^{(2)}=\delta_{g}^{(2)}+v_{\|}^{(2)}-3 \Psi^{(2)}+b_{e} \Delta \ln a^{(2)}+\partial_{\|} \Delta x_{\|}^{(2)}+\frac{2}{\bar{\chi}} \Delta x_{\|}^{(2)}-2 \kappa^{(2)}+\left(\Delta_{g}^{(1)}\right)^{2}-\left(\delta_{g}^{(1)}\right)^{2}-\left(\Phi^{(1)}\right)^{2}-\left(v_{\|}^{(1)}\right)^{2} \\
& +2 \Phi^{(1)} v_{\|}^{(1)}-7\left(\Psi^{(1)}\right)^{2}-\frac{1}{\mathcal{H}^{2}}\left(\partial_{\|} v_{\|}^{(1)}\right)^{2}-\frac{1}{\mathcal{H}^{2}}\left(\Psi^{(1) \prime}\right)^{2}-2 \Phi^{(1)} \Psi^{(1)}-2\left|\gamma^{(1)}\right|^{2}-2\left(\kappa^{(1)}\right)^{2}+\vartheta_{i j}^{(1)} \vartheta^{i j(1)} \\
& -\frac{2}{\mathcal{H}} \Phi^{(1)} \Psi^{(1) \prime}-\frac{2}{\mathcal{H}} v_{\|}^{(1)} \partial_{\|} v_{\|}^{(1)}+\frac{2}{\mathcal{H}} v_{\|}^{(1)} \Psi^{(1) \prime}+\frac{2}{\mathcal{H}} \Psi^{(1)} \partial_{\|} v_{\|}^{(1)}-\frac{2}{\mathcal{H}} \Psi^{(1)} \Psi^{(1) \prime}+\frac{2}{\mathcal{H}^{2}} \Psi^{(1) \prime} \partial_{\|} v_{\|}^{(1)}+\frac{2}{\mathcal{H}} \Phi^{(1)} \partial_{\|} v_{\|}^{(1)} \\
& +v_{\perp i}^{(1)} v_{\perp}^{i(1)}+2 \partial_{\|}\left(+3 \Psi^{(1)}-v_{\|}^{(1)}-\delta_{g}^{(1)}\right) T^{(1)}-\frac{4}{\bar{\chi}} \kappa^{(1)} T^{(1)}-\frac{2}{\bar{\chi}^{2}}\left(T^{(1)}\right)^{2} \\
& +\frac{2}{\mathcal{H}}\left(-\partial_{\|} v_{\|}^{(1)}-\mathcal{H} v_{\|}^{(1)}-\partial_{\|} \Phi^{(1)}+3 \frac{\mathrm{d}}{\mathrm{d} \bar{\chi}} \Psi^{(1)}-\frac{\mathrm{d}}{\mathrm{d} \bar{\chi}} \delta_{g}^{(1)}-\frac{2}{\bar{\chi}^{2}} T^{(1)}-\frac{2}{\bar{\chi}} \kappa^{(1)}\right) \Delta \ln a^{(1)} \\
& \left.+2 \frac{\mathcal{H}^{\prime}}{\mathcal{H}^{2}}\left(\Phi^{(1)}-v_{\|}^{(1)}+\Psi^{(1)}-\frac{1}{\mathcal{H}} \partial_{\|} v_{\|}^{(1)}+\frac{1}{\mathcal{H}} \Psi^{(1) \prime}\right) \Delta \ln a^{(1)}+\left(-b_{e}+\frac{\mathrm{d} \ln b_{e}}{\mathrm{~d} \ln \bar{a}}-\left(\frac{\mathcal{H}^{\prime}}{\mathcal{H}^{2}}\right)^{2}-\frac{2}{\bar{\chi}^{2} \mathcal{H}^{2}}\right)^{\left(\Delta \ln a^{(1)}\right.}\right)^{2} \\
& -2 \partial_{\perp i}\left(v_{\|}^{(1)}-3 \Psi^{(1)}+\delta_{g}^{(1)}\right) \int_{0}^{\bar{\chi}} \mathrm{d} \tilde{\chi}\left[(\bar{\chi}-\tilde{\chi}) \tilde{\partial}_{\perp}^{i}\left(\Phi^{(1)}+\Psi^{(1)}\right)\right]+2 v_{\perp}^{i(1)} \partial_{\perp i} T^{(1)} \\
& +4 S_{\perp}^{i(1)}\left\{-\frac{1}{\bar{\chi}} \int_{0}^{\bar{\chi}} \mathrm{d} \tilde{\chi}\left[(\bar{\chi}-\tilde{\chi}) \tilde{\partial}_{\perp i}\left(\Phi^{(1)}+\Psi^{(1)}\right)\right]+\partial_{\perp i}\left(\frac{1}{\mathcal{H}} \Delta \ln a^{(1)}+T^{(1)}\right)\right\}+2 v_{\perp i o}^{(1)} v_{\perp o}^{i(1)} \\
& -2 v_{\perp i o}^{(1)}\left\{\bar{\chi} \partial_{\perp}^{i}\left(v_{\|}^{(1)}-3 \Psi^{(1)}+\delta_{g}^{(1)}\right)+2 S_{\perp}^{i(1)}+\partial_{\perp}^{i}\left(\frac{1}{\mathcal{H}} \Delta \ln a^{(1)}+T^{(1)}\right)-\frac{1}{\bar{\chi}} \int_{0}^{\bar{\chi}} \mathrm{d} \tilde{\chi}\left[(\bar{\chi}-\tilde{\chi}) \tilde{\partial}_{\perp}^{i}\left(\Phi^{(1)}+\Psi^{(1)}\right)\right]\right\} \\
& -2 \frac{\bar{a}^{2}}{\mathcal{H} \bar{\rho}_{m}}\left(\mathcal{E}_{m}^{\|(1)}-\frac{\mathcal{H}}{\bar{a}^{2}} \bar{\rho}_{m} b_{m} v_{\|}^{(1)}\right)\left[\Phi^{(1)}-v_{\|}^{(1)}+\Psi^{(1)}-\frac{1}{\mathcal{H}} \partial_{\|} v_{\|}^{(1)}+\frac{1}{\mathcal{H}} \Psi^{(1) \prime}-\left(1+\frac{\mathcal{H}^{\prime}}{\mathcal{H}^{2}}\right) \Delta \ln a^{(1)}\right] \\
& -\left(\frac{\bar{a}^{2}}{\mathcal{H} \bar{\rho}_{m}}\right)^{2}\left(\mathcal{E}_{m}^{\|(1)}-\frac{\mathcal{H}}{\bar{a}^{2}} \bar{\rho}_{m} b_{m} v_{\|}^{(1)}\right)^{2} \text {. }
\end{aligned}
$$

[1] J. Yoo, A. L. Fitzpatrick and M. Zaldarriaga, Phys. Rev. D 80, 083514 (2009) arXiv:0907.0707.

[2] J. Yoo, Phys. Rev. D 82, 083508 (2010) arXiv:1009.3021.

[3] C. Bonvin and R. Durrer, Phys. Rev. D 84, 063505 (2011) arXiv:1105.5280.

[4] A. Challinor and A. Lewis, Phys. Rev. D 84, 043516 (2011) arXiv:1105.5292.

[5] D. Jeong, F. Schmidt and C. M. Hirata, Phys. Rev. D 85, 023504 (2012) arXiv:1107.5427.

[6] M. Bruni, R. Crittenden, K. Koyama, R. Maartens, C. Pitrou and D. Wands, Phys. Rev. D 85 (2012) 041301 arXiv:1106.3999.

[7] F. Schmidt and D. Jeong, Phys. Rev. D 86, 083527 (2012) arXiv:1204.3625.

[8] D. Bertacca, R. Maartens, A. Raccanelli and C. Clarkson, JCAP 1210 (2012) 025 arXiv:1205.5221.

[9] R. Maartens, G. -B. Zhao, D. Bacon, K. Koyama and A. Raccanelli, JCAP 1302 (2013) 044 arXiv:1206.0732.

[10] J. Yoo, N. Hamaus, U. Seljak and M. Zaldarriaga, Phys. Rev. D 86 (2012) 063514 arXiv:1206.5809.

[11] A. Hall, C. Bonvin and A. Challinor, Phys. Rev. D 87 (2013) 064026 arXiv:1212.0728.

[12] L. Lombriser, J. Yoo and K. Koyama, Phys. Rev. D 87 (2013) 104019 arXiv:1301.3132.

[13] J. Yoo and V. Desjacques, Phys. Rev. D 88, 023502 (2013) arXiv:1301.4501.

[14] D. Jeong and F. Schmidt, arXiv:1305.1299.

[15] D. Duniya, D. Bertacca and R. Maartens, JCAP 1310 (2013) 015 arXiv:1305.4509.

[16] A. Raccanelli, D. Bertacca, O. Doré and R. Maartens, JCAP, to appear arXiv:1306.6646].

[17] E. Di Dio, F. Montanari, J. Lesgourgues and R. Durrer, JCAP 1311, 044 (2013) $\operatorname{arXiv:1307.1459}$.

[18] E. Di Dio, F. Montanari, R. Durrer and J. Lesgourgues, JCAP 1401, 042 (2014) arXiv:1308.6186.

[19] A. Raccanelli, D. Bertacca, R. Maartens, C. Clarkson and O. Dor, Gen. Rel. Grav. 48 (2016) no.7, 84 arXiv:1311.6813.

[20] D. Bertacca, R. Maartens and C. Clarkson, JCAP 1409 (2014) no.09, 037 arXiv:1405.4403.

[21] T. Pyne and S. M. Carroll, Phys. Rev. D 53 (1996) 2920 astro-ph/9510041

[22] S. Mollerach and S. Matarrese, Phys. Rev. D 56 (1997) 4494 astro-ph/9702234.

[23] O. Umeh, C. Clarkson and R. Maartens, Class. Quant. Grav. 31 (2014) 202001 arXiv:1207.2109.

[24] I. Ben-Dayan, G. Marozzi, F. Nugier and G. Veneziano, JCAP 1211, 045 (2012) arXiv:1209.4326.

[25] G. Fanizza, M. Gasperini, G. Marozzi and G. Veneziano, JCAP 1311, 019 (2013) arXiv:1308.4935.

[26] O. Umeh, C. Clarkson and R. Maartens, Class. Quant. Grav. 31 (2014) 205001 arXiv:1402.1933.

[27] D. Bertacca, Class. Quant. Grav. 32 (2015) no.19, 195011 arXiv:1409.2024. 
[28] J. T. Nielsen and R. Durrer, arXiv:1606.02113.

[29] I. Ben-Dayan, M. Gasperini, G. Marozzi, F. Nugier and G. Veneziano, Phys. Rev. Lett. 110, 021301 (2013) arXiv:1207.1286.

[30] I. Ben-Dayan, M. Gasperini, G. Marozzi, F. Nugier and G. Veneziano, JCAP 1306, 002 (2013) arXiv:1302.0740.

[31] I. Ben-Dayan, R. Durrer, G. Marozzi and D. J. Schwarz, Phys. Rev. Lett. 112 (2014) 221301 arXiv:1401.7973.

[32] C. Clarkson, O. Umeh, R. Maartens and R. Durrer, JCAP 1411 (2014) no.11, 036 arXiv:1405.7860.

[33] F. Bernardeau, C. Bonvin and F. Vernizzi, Phys. Rev. D 81 (2010) 083002 arXiv:0911.2244.

[34] F. Bernardeau, C. Bonvin, N. Van de Rijt and F. Vernizzi, Phys. Rev. D 86 (2012) 023001 arXiv:1112.4430.

[35] C. -P. Ma and E. Bertschinger, Astrophys. J. 455 (1995) 7 astro-ph/9506072.

[36] S. Matarrese, S. Mollerach and M. Bruni, Phys. Rev. D 58 (1998) 043504 astro-ph/9707278.

[37] H. Kodama and M. Sasaki, Prog. Theor. Phys. Suppl. 78 (1984) 1.

[38] N. Bartolo, S. Matarrese and A. Riotto, JCAP 1104 (2011) 011 arXiv:1011.4374.

[39] D. Bertacca, N. Bartolo, C. Clarkson, R. Maartens, S. Matarrese, A. Raccanelli, "The relativistic galaxy bispectrum", in preparation. 